Universidade de São Paulo

Instituto de Física

\title{
Limite de Altas Temperaturas em Presença de Campos Externos Estáticos
}

\author{
João Bosco de Siqueira \\ Orientador: Prof. Dr. Fernando Tadeu Caldeira Brandt \\ Tese de doutorado apresentada ao \\ Instituto de Física visando a obtenção \\ do título de Doutor em Ciências
}

Banca Examinadora:

Prof. Dr. Fernando Tadeu Caldeira Brandt (IF-USP)

Prof. Dr. Marcelo Otávio Caminha Gomes (IF-USP)

Prof. Dr. Paulo Teotônio Sobrinho (IF-USP)

Prof. Dr. Ademir Eugênio de Santana (IF-UnB)

Prof. Dr. Adolfo Pedro Carvalho Malbouisson (CBPF)

São Paulo 
FICHA CATALOGRÁFICA

Preparada pelo Serviço de Biblioteca e Informação do Instituto de Física da Universidade de São Paulo

Siqueira, João Bosco de

Limite de altas temperaturas em presença de campos externos estáticos. - São Paulo, 2013.

Tese (Doutorado) - Universidade de São Paulo. Instituto de Física - Depto. de Física Experimental

Orientador: Prof. Dr. Fernando Tadeu Caldeira Brandt

Área de Concentração: Física

Unitermos: 1. Teoria de Campos; 2. Física Teórica; 3. Teoria Quântica de Campo. 
Aos meus amados sobrinhos Júlia e Davi, dedico este trabalho. 


\section{Agradecimentos}

Em primeiro lugar agradeço ao meu orientador e amigo Prof. Fernando Brandt pelo apoio e por me aturar desde o mestrado, nesses seis anos em que estamos trabalhando juntos. Obrigado por tudo Fernando, eu aprendi muito e sou muito grato por tudo.

Agradeço ao Prof. Josif Frenkel pela colaboração e por todas as verdadeiras aulas de física que foram as conversas com este grande mestre.

Agradeço a minha família, em especial a minha mãe Ângela, minhas irmãzinhas do meu coração Tatiana e Thaís e meu cunhadinho Júlio, pelo apoio. Obrigado de coração, amo vocês.

Mas sem dúvida, o agradecimento mais especial vai para a minha esposa Ana Cláudia, por me aguentar nos momentos difíceis e compartilhar as alegrias desta montanha russa cheia de emoções chamada doutoramento em ciências. Obrigado amor.

Agradeço ao CNPQ pelo fomento. 


\section{Resumo}

Investigamos certas propriedades físicas da teoria de campos à temperatura finita. Mostramos que, na presença de campos externos bosônicos, o limite de altas temperaturas das funções de Green pode ser obtido supondo que todas as componentes dos momentos externos são nulas, para todas as funções de Green 1PI em ordem de um laço e em ordem de dois laços para a auto-energia. No contexto do método do campo de fundo, este resultado implica que a lagrangiana efetiva estática de um campo externo bosônico pode ser obtida calculando a pressão na presença de um campo externo independente do espaço-tempo, no limite de altas temperaturas. Aplicamos este resultado para obter a lagrangiana efetiva estática de um campo de Maxwell interagindo com um plasma da QED em ordem de dois laços. Obtivemos também a lagrangiana efetiva estática de um campo gravitacional interagindo com um campo escalar real livre, e com um plasma da QED em ordem de dois laços. 


\section{Abstract}

We have investigated some physical proprieties of finite temperature field theory. We have shown that, in the presence of an external bosonic field, the high temperature limit of the static Green's functions can be obtained assuming that all components of the external momenta are zero. This was shown to be the case for all 1PI Green's functions, at one-loop order, as well as for the self-energy, at two-loop order. In the context of the background field method, this result implies that the static effective Lagrangian of an external field can be obtained computing the pressure in the presence of an external field which is independent of the space-time, in the high temperature limit. We have applied this result in order to obtain the effective Lagrangian of an external Maxwell field interacting with a QED plasma at two-loop order. We have also obtained the effective Lagrangian of the gravitational field interacting with a free neutral scalar field, as well as with a QED plasma at two-loop order. 


\section{Sumário}

$\begin{array}{lll}1 & \text { Introdução } & \text { p. } 1\end{array}$

2 Conceitos básicos $\quad$ p. 5

2.1 Mecânica estatística quântica $\ldots \ldots \ldots \ldots \ldots \ldots \ldots \ldots \ldots$

$2.1 .1 \quad$ A equação de Liouville $\ldots \ldots \ldots \ldots \ldots \ldots \ldots \ldots$

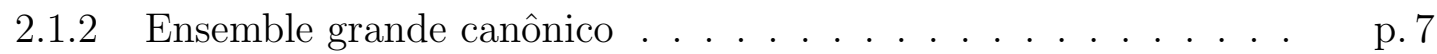

2.1 .3 Gás ideal quântico $\ldots \ldots \ldots \ldots \ldots \ldots$. . . . . . . . . 10

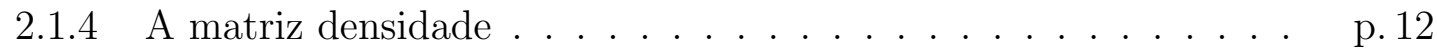

2.2 Função de partição no formalismo funcional $\ldots \ldots \ldots \ldots \ldots \ldots$ p. 18

$2.2 .1 \quad$ O formalismo da integração funcional $\ldots \ldots \ldots \ldots \ldots \ldots$. . . . 19

2.2 .2 A função de partição para o campo escalar real livre. . . . . . . p p. 25

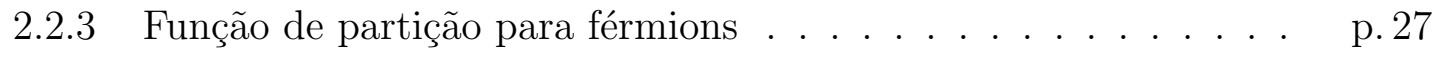

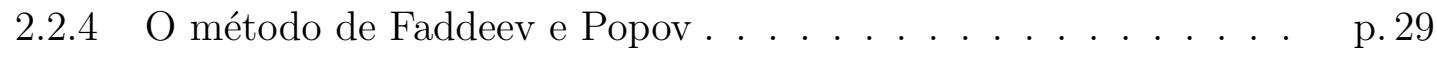

2.2 .5 Função de partição para o campo de Maxwell $\ldots \ldots \ldots \ldots$. . . . 32

3 Campo escalar a temperatura finita $\quad$ p. 35

3.1 A função de partição para o campo escalar real livre. . . . . . . . . . . p. 35

3.2 Interações e teoria de perturbação $\ldots \ldots \ldots \ldots \ldots \ldots \ldots$. . . . . . 40

$3.3 \quad$ A pressão em termos de diagramas conexos $\ldots \ldots \ldots \ldots \ldots$. . . . . . 46

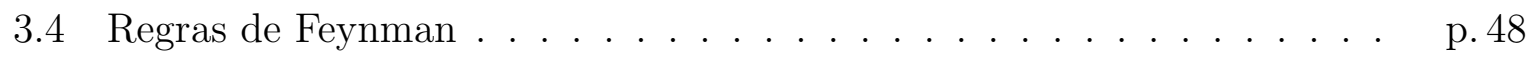

$3.5 \quad$ Primeira correção para a pressão $\ldots \ldots \ldots \ldots \ldots \ldots \ldots \ldots$

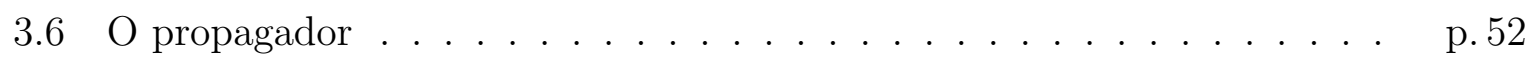




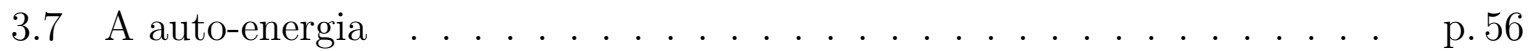

3.8 Soma das divergências infravermelhas . . . . . . . . . . . . . . . . . . p. 59

$3.8 .1 \quad$ Caso $d$ par $\ldots \ldots \ldots \ldots \ldots$ p. 61

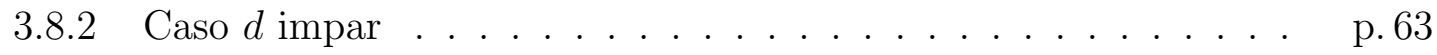

4 Eletrodinâmica Quântica a Temperatura Finita em $\boldsymbol{d}$ dimensões $\quad$ p. 65

4.1 A função de partição da Eletrodinâmica Quântica . . . . . . . . . . . . p. 65

4.2 Pressão ordem de um laço . . . . . . . . . . . . . . . . . p 66

4.3 Pressão ordem de dois laços $\ldots \ldots \ldots$. . . . . . . . . . . . . . . 69

4.4 Auto-energia dos fótons $\ldots \ldots \ldots \ldots$ p. 72

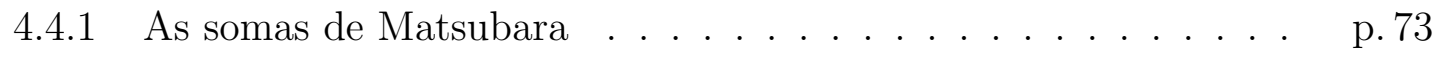

4.4 .2 O limite de altas temperaturas . . . . . . . . . . . p. 75

$4.4 .3 \quad$ Base tensorial transversal $\ldots \ldots \ldots$. . . . . . . . . . . . 79

$4.4 .4 \quad$ Limites estático e de grandes comprimentos de onda . . . . . . . $\quad$ p. 84

$4.4 .5 \quad$ Expressão analítica $\ldots \ldots \ldots$. . . . . . . . . . . . . . . . p. 87

$4.5 \quad$ A soma das divergências infravermelhas para a pressão $\quad \ldots . . \quad$. . . p. 88

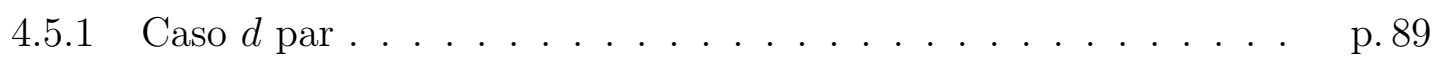

$4.5 .2 \quad$ Caso $d$ ímpar $\ldots \ldots \ldots \ldots$. . . . . . . . . . . . . . . . . . 91

4.6 Auto-energia dos elétrons $\ldots \ldots \ldots \ldots$. . . . . . . . . . . . . . . . . . . .

$4.6 .1 \quad$ Soma de Matsubara . . . . . . . . . . . . . . . . . . p. 94

$4.6 .2 \quad$ A região dos "Hard Thermal Loops" . . . . . . . . . . . . . . p.96

4.6 .3 Integração radial $\ldots \ldots \ldots$. . . . . . . . . . . . . . . . . . 97

4.6 .4 Integração angular $\ldots \ldots \ldots$. . . . . . . . . . . . . . . . . 99

4.6 .5 Limite estático . . . . . . . . . . . . . . . . p. 100

4.6 .6 Limite de grandes comprimentos de onda . . . . . . . . . . . p. 101

4.6 .7 Invariância de calibre . . . . . . . . . . . . . . . . p. 102

4.7 Função de vértice $\ldots \ldots \ldots$. . . . . . . . . . . . . . . . . . . . . . . . . . . . $\quad$ p. 102 
4.8 Identidades de Ward $\ldots \ldots \ldots \ldots \ldots \ldots$

4.9 Lagrangiana efetiva térmica $\ldots \ldots \ldots \ldots \ldots$. . . . . . . . . . . 111

5 O limite de altas temperaturas em campos de fundo estáticos $\quad$ p.113

$5.1 \quad$ O método do campo de fundo $\ldots \ldots \ldots \ldots$. . . . . . . . . . . . . . . . .

5.2 Limite de altas temperaturas das funções de Green estáticas $\ldots . . \quad$ p. 115

$5.2 .1 \quad$ Auto-energia em ordem um laço . . . . . . . . . . . . . . p. 115

$5.2 .2 \quad$ Procedimento iterativo para amplitudes em um laço . . . . . . . p. 119

5.3 Auto-energia em ordem dois laços . . . . . . . . . . . . . . . p. 125

5.3 .1 Primeira topologia $\ldots \ldots \ldots \ldots \ldots \ldots$. . . . . . . . 126

5.3 .2 Segunda topologia $\ldots \ldots \ldots \ldots$. . . . . . . . . . . . . . 129

6 QED em um campo de Maxwell externo e estático p.133

6.1 QED em um campo eletromagnético externo . . . . . . . . . . . . p. 133

6.1 .1 Ordem um laço . . . . . . . . . . . . . . . . p. 133

6.1 .2 Ordem de dois laços . . . . . . . . . . . . . . . p. 137

7 Campos térmicos na presença de um campo gravitacional p. 143

7.1 Campo escalar livre . . . . . . . . . . . . . . . . . . . . p. 143

7.1 .1 Método perturbativo . . . . . . . . . . . . . . p. 143

7.1 .2 Métrica estática geral . . . . . . . . . . . . . . . p. 147

7.2 QED em um campo gravitacional estático $\ldots \ldots \ldots \ldots$. . . . . . . . . 151

7.2 .1 Definição do modelo . . . . . . . . . . . . . . . . p. 151

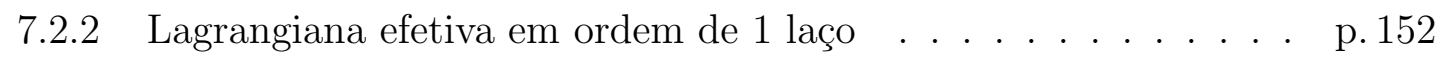

7.2 .3 Lagrangiana efetiva em ordem de 2 laços . . . . . . . . . . . . . p. 155

\begin{tabular}{llr}
\hline C Conclusão & p. 159
\end{tabular}

\begin{tabular}{|ll}
\hline Apêndice A - Integrais funcionais & p. 161
\end{tabular} 


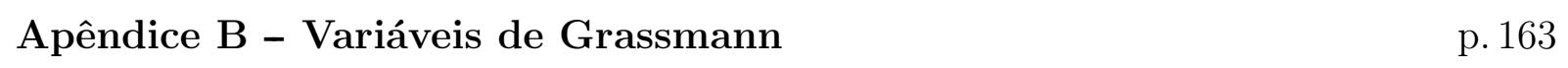

B.1 Integrais gaussianas . . . . . . . . . . . . . . . . . p. 163

B.2 $\quad$ Integrais de trajetória para um grau de liberdade fermiônico $\ldots$. . . . p p. 166

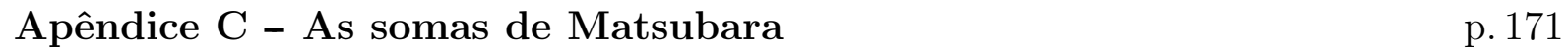

\begin{tabular}{|l|l|l|}
\hline Apêndice D - Integral escalar para a função de dois pontos & p. 177
\end{tabular}

Apêndice E - Limite de altas temperaturas das funções de Green

$\begin{array}{lr}\text { estáticas } & \text { p. } 179\end{array}$

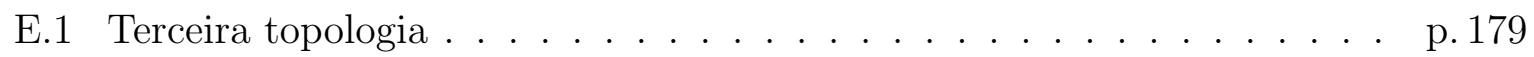

E.2 Quarta topologia . . . . . . . . . . . . . . . . . p. 180

E.3 Quinta topologia . . . . . . . . . . . . . . . . . . . . . . . . . . p. 182

Apêndice F - Obtenção da auto-energia estática do fóton a partir da

$\begin{array}{ll}\text { ação efetiva } & \text { p. } 185\end{array}$

F.1 Auto-energia em ordem de um laço . . . . . . . . . . . . . . p. 185

F.2 Auto-energia em ordem dois laços $\ldots \ldots \ldots$. . . . . . . . . . 187

Apêndice G - Alguns resultados básicos sobre campos em um espaço$\begin{array}{ll}\text { tempo curvo } & \text { p. } 191\end{array}$

G.1 Representações do grupo de Lorentz. . . . . . . . . . . . . . . . . . . . p. 191

G.1.1 Campo de Dirac . . . . . . . . . . . . . . . . . . . p. 194

G.2 O formalismo das tetradas $\ldots \ldots \ldots \ldots$. . . . . . . . . . . . . 198

G.3 Derivada covariante para representações do grupo de Lorentz . . . . . . p. 203

\begin{tabular}{|lll}
\hline Apêndice H - Funções de Green do campo gravitacional & p. 207
\end{tabular}

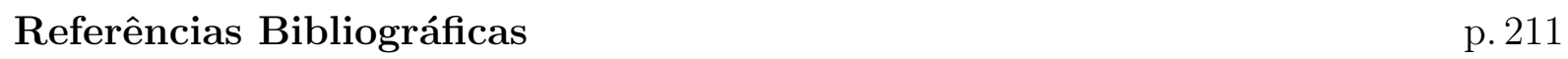




\section{Introdução}

A teoria de campos à temperatura finita é certamente um formalismo adequado para tratar problemas de muitos corpos para sistemas quânticos e relativísticos. Situada no encontro entre a mecânica estatística e a teoria quântica de campos, a teoria de campos à temperatura finita constitui um arcabouço teórico essencialmente interdisciplinar, que encontra aplicação em diversos fenômenos entre os quais podemos destacar a colisão de íons pesados [Won95], e fenômenos térmicos na presença de um campo gravitacional, como no universo primordial ou na presença de objetos astrofísicos massivos [KG06, Wei08].

Existem três formalismos para abordar os diversos problemas em teoria de campos à temperatura finita. O formalismo do tempo imaginário [Bel96, KG06], o formalismo do tempo real [Das97] e a dinâmica dos campos térmicos [KMMS09]. Para os problemas que investigamos no presente estudo, envolvendo campos externos estáticos, utilizaremos o formalismo do tempo imaginário.

Em teorias de campos, as ações efetivas são importantes objetos que geram todas as funções de Green 1Pل1 da teoria. Para obter expressões fechadas para as ações efetivas, muitas vezes é conveniente separar o campo em uma parte clássica e uma parte de flutuações quânticas, e supor que as fontes e a dinâmica do campo clássico são exteriores ao sistema em questão. Esta separação é chamada de método do campo de fundo. No contexto do método do campo de fundo, podemos identificar o funcional gerador das funções de Green conexas como função do campo de fundo com a ação efetiva como funcional do campo externo Abb82]. Portanto, a partir do cálculo de diagramas de vácuo conexos na presença do campo de fundo, podemos obter todas as funções de Green 1PI da teoria.

À temperatura finita, o funcional gerador das funções de Green conexas está relacionado com a pressão. Logo, no método do campo de fundo, podemos obter a lagrangiana efetiva térmica da teoria computando diagramas de vácuo para pressão na presença de um campo externo. Até onde sabemos, este resultado foi pouco explorado neste contexto.

\footnotetext{
${ }^{1}$ Usaremos a sigla em inglês "one particle irreducible".
} 
No limite de altas temperaturas, as amplitudes térmicas são dominadas pela região dos "Hard Thermal Loops", na qual os momentos externos são muito menores que os momentos de integração . Nesta região, as funções de Green de $(n+1)$ e de $n$-pontos estão relacionadas por meios de identidades de Ward simples, tanto para as teorias de calibre abelianas, quanto para o caso não abeliano. Isso indica que essas funções de Green devem ser geradas por uma ação efetiva invariante de calibre. De fato, estas ações efetivas são conhecidas em ordem de um laço e são funcionais não locais e invariantes de calibre do campo externo [BP90a, BP90b, FT90, FT92, Bel96].

Quando as interações gravitacionais estão presentes, há uma simetria de calibre adicional, associada ao campo do gráviton, que é induzida pela invariância por transformações gerais de coordenadas. Na região dos "Hard Thermal Loops" as funções de Green do campo do gráviton também estão relacionadas por meio de identidades de Ward, o que indica que deve haver uma ação efetiva invariante por transformações gerais de coordenadas que gere estas amplitudes. Entretanto, apenas quando o campo gravitacional é estático é que a forma desta ação efetiva é conhecida, em ordem de um laço [Reb91, BF93b, BF93a, BFT95, BS12a].

Já era conhecido o fato de que na presença de campos externos bosônicos, podemos computar o limite de altas temperaturas das funções de Green estáticas supondo que todas as componentes do momento externo são nulas, para as funções de dois e três pontos em ordem de um laço [FPT09]. Recentemente mostramos que esta identidade também é válida para todas as funções de Green 1PI em ordem de um laço e em ordem de dois laços para a auto-energia [BS12b], e futuramente analisaremos a validade desta identidade para todas as ordens.

Aplicada ao método do campo de fundo, esta identidade implica que podemos obter a lagrangiana efetiva estática de um campo de fundo bosônico computando a pressão na presença de configurações constantes do campo externo, no limite de altas temperaturas. Usando este método calculamos a lagrangiana efetiva de um campo de Maxwell estático interagindo com um plasma da QED2 2 até ordem de dois laços. Obtivemos também a lagrangiana efetiva de um campo gravitacional externo e estático interagindo com campos térmicos. Primeiramente, consideramos um campo escalar livre interagindo com um campo gravitacional externo [BFS12]. Em seguida obtivemos a lagrangiana efetiva estática do campo gravitacional interagindo com um plasma da QED até a ordem de dois laços.

O trabalho está organizado da seguinte forma:

\footnotetext{
${ }^{2}$ Usaremos a sigla em inglês "Quantum Electrodynamics".
} 
No capítulo 2 faremos uma breve revisão dos conceitos de mecânica estatística que serão usados no decorrer do texto. Em seguida introduziremos o método funcional em teoria de campos e obteremos a representação da função de partição no formalismo funcional.

No capítulo 3 estudaremos o campo escalar com auto interação $\phi^{4}$ a temperatura finita e iremos generalizar os resultados perturbativos conhecidos na literatura [KG06, Bel96] para um espaço-tempo de $d$ dimensões. É conhecido que as divergências infravermelhas introduzem uma não-analiticidade na série perturbativa para a pressão. Nossa contribuição neste contexto será mostrar que o caráter não analítico da série perturbativa depende qualitativamente da dimensionalidade do espaço-tempo $d$. Se $d$ for par a não-analiticidade é do tipo $\lambda^{(d-1) / 2}$, enquanto se $d$ for impar a não-analiticidade é apenas logarítmica [BFS12, onde $\lambda$ é a constante de acoplamento. No capítulo 4 o mesmo será feito no contexto da QED.

Faremos uma breve revisão do método do campo de fundo no capítulo 5, e veremos como o funcional gerador das funções de Green 1PI do campo de fundo esta relacionado com o funcional gerador das funções de Green conexas como função do campo externo. Deduziremos a identidade que relaciona as funções de Green estáticas com as de momento externo nulo, no limite de altas temperaturas para todas as funções de Green 1PI em ordem de um laço e em ordem de dois laços para a auto-energia. Assim, veremos que podemos obter a lagrangiana efetiva térmica de um campo de fundo calculando a pressão na presença de um campo externo independente do espaço-tempo, no limite de altas temperaturas.

A título de aplicação deste resultado, no capítulo 6 obteremos a lagrangiana efetiva de um campo de fundo de Maxwell estático interagindo com um plasma da QED até ordem de dois laços. No capítulo 7 consideraremos campos térmicos na presença um campo gravitacional estático. Obteremos a lagrangiana efetiva estática do campo gravitacional interagindo com um campo escalar térmico em ordem de um laço. Obteremos também a lagrangiana efetiva estática do campo gravitacional interagindo com um plasma da QED até ordem de dois laços.

Exceto na seção 2.1, usaremos o sistema de unidades em que $k_{B}=c=\hbar=1$. Usaremos uma métrica com signatura $(+,-, \cdots,-)$. 


\section{Conceitos básicos}

Neste capítulo estudaremos conceitos de mecânica estatística e do formalismo da integração funcional que serão fundamentais em todo trabalho.

\subsection{Mecânica estatística quântica}

Neste seção iremos enunciar alguns resultados da mecânica estatística quântica que serão importantes no decorrer do trabalho. Iremos primeiramente deduzir a equação de Liouville para motivar o postulado fundamental da mecânica estatística, que será enunciado na sequência. Depois iremos utilizar o postulado para construir o ensemble grande canônico e veremos como se dá a sua conexão com a termodinâmica. Como exemplo, iremos obter a função de partição de um gás ideal quântico, com as estatísticas de Fermi-Dirac e de Bose-Einstein. Em seguida, construiremos o formalismo da matriz densidade, que certamente é um formalismo adequado para tratar sistemas térmicos no contexto da mecânica quântica, e diferenciaremos os conceitos de estado puro e de mistura estatística. Escreveremos a matriz densidade do ensemble grande canônico, para um sistema em equilíbrio com temperatura e potencial químico fixos, que consiste no objeto fundamental para o cálculo de valores esperados térmicos no decorrer de todo o trabalho.

\subsubsection{A equação de Liouville}

Considere um sistema clássico com $N$ graus de liberdade na descrição hamiltoniana. O estado físico do sistema é então determinado pelas $N$ coordenadas generalizadas $q^{i}$ e seus respectivos momentos canonicamente conjugados $p_{i}$ com $i=1, \cdots, N$. O espaço de fase deste sistema é o conjunto de pontos $\left(q^{i}, p_{i}\right)$ acessíveis ao sistema, que é um espaço 
$2 N$-dimensional. A evolução temporal deste sistema é ditada pelas equações de Hamilton

$$
\begin{aligned}
\dot{q}^{i} & =\frac{\partial H}{\partial p_{i}} \\
\dot{p}_{i} & =-\frac{\partial H}{\partial q^{i}}
\end{aligned}
$$

onde $H=H\left(q^{i}, p_{i}, t\right)$ é a hamiltoniana do sistema, e o terceiro argumento denota uma possível dependência explícita no tempo.

Definimos a densidade de estados acessíveis ao sistema na região do espaço de fase entre $\left(q^{i}, p_{i}\right)$ e $\left(q^{i}+\delta q^{i}, p_{i}+\delta p_{i}\right)$ no em um dado tempo $t$ por $\rho\left(q^{i}, p_{i}, t\right)$. Assim,

$$
\rho\left(q^{i}, p_{i}, t\right) \delta q \delta p
$$

é o número de estados contidos no hipervolume infinitesimal $\delta q \delta p=\delta q^{1} \delta p_{1} \cdots \delta q^{N} \delta p_{N}$ do espaço de fase.

O determinismo da mecânica clássica afirma que dada uma condição inicial $\left[q^{i}\left(t_{0}\right), p_{i}\left(t_{0}\right)\right]$, as equações de Hamilton (2.1) fixam unicamente o estado do sistema $\left[q^{i}(t), p_{i}(t)\right]$ para todos os tempos posteriores, o que implica que as trajetórias no espaço de fase não se cruzan 11. Logo não existem fontes nem sumidouros para trajetórias no espaço de fase, de tal manira que $\rho\left(q^{i}, p_{i}, t\right)$ deve satisfazer a equação da continuidade

$$
\frac{\partial \rho}{\partial t}+\dot{q}^{i} \frac{\partial \rho}{\partial q^{i}}+\dot{p}_{i} \frac{\partial \rho}{\partial p_{i}}=0
$$

Por outro lado, a derivada total da densidade de estados $\rho$ em relação ao tempo possui a forma

$$
\begin{aligned}
\frac{d \rho}{d t} & =\frac{\partial \rho}{\partial t}+\dot{q}^{i} \frac{\partial \rho}{\partial q^{i}}+\dot{p}_{i} \frac{\partial \rho}{\partial p_{i}} \\
& =\frac{\partial \rho}{\partial t}+\frac{\partial \rho}{\partial q^{i}} \frac{\partial H}{\partial p_{i}}-\frac{\partial \rho}{\partial p_{i}} \frac{\partial H}{\partial q^{i}} \\
& =\frac{\partial \rho}{\partial t}+\{\rho, H\}
\end{aligned}
$$

onde usamos as equações de Hamilton (2.1). Os parênteses de Poisson são definidos como

$$
\{\rho, H\}=\frac{\partial \rho}{\partial q^{i}} \frac{\partial H}{\partial p_{i}}-\frac{\partial \rho}{\partial p_{i}} \frac{\partial H}{\partial q^{i}} .
$$

Deste modo, comparando (2.3) com 2.4 obtemos

$$
\frac{d \rho}{d t}=\frac{\partial \rho}{\partial t}+\{\rho, H\}=0
$$

\footnotetext{
${ }^{1}$ Desde que consigamos distinguir estados com precisão infinita.
} 
que é a equação de Liouville

$$
\frac{\partial \rho}{\partial t}=\{H, \rho\}
$$

As equações de Hamilton podem ser sintetizados usando os parênteses de Poisson. De fato, 2.1 podem ser resumidas em

$$
\frac{d f(q, p)}{d t}=\{f(q, p), H\}
$$

para uma função $f$ que depende do tempo apenas através de $q$ e $p$. Portanto, a equação (2.8) implica que a evolução temporal é gerada pela hamiltoniana. Logo, quando a hamiltoniana não depende explicitamente do tempo, temos que o "escoamento" no espaço de fase descrito pela densidade de estados $\rho$ será estacionário pois as trajetórias possíveis não se deformarão com o tempo. Assim, para sistemas de energia fixa teremos

$$
\frac{\partial \rho}{\partial t}=0
$$

e portanto

$$
\{H, \rho\}=0,
$$

$\rho$ é uma constante de movimento. Ou seja, $\rho$ depende de $q$ e $p$ apenas através da hamiltoniana $H$ Sal97].

\subsubsection{Ensemble grande canônico}

Um sistema macroscópico, tal como um gás no interior de um bulbo, possui um número de constituintes da ordem de $10^{23}$. Logo, é inviável descrever este sistema em termos de seus microestados, que são os estados do espaço de fase deste sistema. Assim, no equilíbrio, descrevemos o estado físico sistemas macroscópicos em termos de variáveis termodinâmicas, ou seja, do estado macroscópico do sistema (macroestado).

Como vimos na seção anterior, para um sistema de energia fixa, a densidades de estados acessíveis no espaço de fase é uma constante de movimento. Motivado neste fato, o postulado fundamental da mecânica estatística afirma que para um sistema de energia fixa, todos os microestados acessíveis ao sistema no espaço de fase são igualmente prováveis, que é a hipótese de equiprobabilidades à priori. Esta hipótese expressa nossa ignorância sobre o microestado de um sistema, mesmo quando conhecemos o seu estado macroscópico. 
Logo, a probabilidade de encontrar um sistema físico em uma dada configuração macroscópica deve ser proporcional ao número que estados microscópios que levam à mesma configuração macroscópica. Assim, no equilíbrio, o sistema ira para o macroestado que maximiza o número de microestados acessíveis ao sistema $\Omega$. Lembrando da segunda lei da termodinâmica, que versa sobre a maximização da entropia, temos que a entropia deve ser uma função monotonicamente crescente do número de estados acessíveis ao sistema. Logo, definimos

$$
S=k_{B} \log \Omega
$$

Considere um sistema $s$ em contato com um reservatório térmico $R$, de tal maneira que o sistema e o reservatório podem trocar partículas e energia, mantendo a energia total $E$ e o número de partículas total $N$ constante. Os volumes do sistema e do reservatório são mantidos fixos. Suponha também que a interação entre o sistema e o reservatório é suficientemente fraca de tal maneira que a energia total seja a soma das energias do sistema e do reservatório.

Usando o postulado fundamental da mecânica estatística, temos que a probabilidade $p_{j}$ de encontrar o sistema $s$ em um particular microestado $j$ de energia $E_{j}$ e número de partículas $N_{j}$ será proporcional ao número do microestados acessíveis ao reservatório $R$ com energia $E-E_{j}$ e número de partículas $N-N_{j}$, isto é

$$
p_{j}=C \Omega_{R}\left(E-E_{j}, N-N_{j}\right),
$$

onde $C$ é uma normalização. Deste modo, tomando o logaritmo da equação (2.12) e lembrando da da definição da entropia (2.11), teremos

$$
\log p_{j}=\frac{S_{R}\left(E-E_{j}, N-N_{j}\right)}{k_{B}}+C^{\prime}
$$

Agora vamos supor que o sistema $s$ é muito menor que o reservatório térmico $R$, isto é, $E_{j} \ll E$ e $N_{j} \ll N$. Com isso teremos

$$
\log p_{j} \simeq C^{\prime \prime}+\left.\frac{\partial S_{R}}{\partial E}\right|_{(V, N)} \frac{-E_{j}}{k_{B}}+\left.\frac{\partial S_{R}}{\partial N}\right|_{(E, V)} \frac{-N_{j}}{k_{B}}
$$

para um tratamento mais rigoroso desta aproximação veja [Sal97.

No limite do reservatório térmico ideal, o sistema será então muito pequeno em relação ao reservatório. Portanto, a presença do sistema não deve mudar o potencial químico e 
temperatura do reservatório e assim vemos que

$$
\left.\frac{\partial S_{R}}{\partial E}\right|_{(V, N)}=\frac{1}{T}
$$

e

$$
\left.\frac{\partial S_{R}}{\partial N}\right|_{(E, V)}=-\frac{\mu}{T}
$$

com $T$ e $\mu$ sendo a temperatura e o potencial químico de equilíbrio da interação entre sistema e reservatório, respectivamente. Deste modo, substituindo 2.15 e 2.16 em (2.14), vemos que a probabilidade de encontrar os sistema em um particular microestado $j$ com energia $E_{j}$ e número de partículas $N_{j}$ é dada por

$$
p_{j}=\frac{1}{Z} \exp \left(-\beta E_{j}+\beta \mu N_{j}\right)
$$

onde $\beta=\left(k_{B} T\right)^{-1}$ e a normalização $Z^{-1}$ é soma sobre todos os microestados

$$
Z=\sum_{j} \exp \left(-\beta E_{j}+\beta \mu N_{j}\right)
$$

que é a função de partição do ensemble grande canônico.

A conexão com a termodinâmica é feita impondo que os valores de equilíbrio das grandezas macroscópicas coincidem com as respectivas médias no ensemble. Deste modo para a energia interna do sistema teremos

$$
U=\frac{1}{Z} \sum_{j} E_{j} \exp \left(-\beta E_{j}+\beta \mu N_{j}\right)=-\left.\frac{\partial \log Z}{\partial \beta}\right|_{(\mu, V)}
$$

e o número de partículas

$$
N=\frac{1}{Z} \sum_{j} N_{j} \exp \left(-\beta E_{j}+\beta \mu N_{j}\right)=\left.\frac{\partial \log Z}{\partial \beta \mu}\right|_{(\beta, V)} .
$$

As expressões (2.19) e 2.20 implicam que $\log Z$ é uma transformada de Legendre da entropia

$$
k_{B} \log Z=S-\frac{1}{T} U+\frac{\mu}{T} N=\Phi(T, \mu, V)
$$

que é a função de Massieu. Alternativamente, em termos do potencial termodinâmico de Landau $\Omega$, ou simplesmente energia livre, escrevemos

$$
\log Z=-\beta \Omega=\beta P V,
$$


onde $\Omega$ é uma transformada de Legendre da energia que possui a forma

$$
\Omega(T, V, \mu)=U-T S-\mu N=-P V .
$$

Logo, da equação 2.23 e da equação fundamental da termodinâmica na representação da energia

$$
d U=T d S-P d V+\mu d N
$$

seguem as seguintes derivadas termodinâmicas

$$
\begin{aligned}
& \left.\frac{\partial \Omega}{\partial T}\right|_{V, \mu}=-S \\
& \left.\frac{\partial \Omega}{\partial V}\right|_{T, \mu}=-P \\
& \left.\frac{\partial \Omega}{\partial \mu}\right|_{T, V}=-N .
\end{aligned}
$$

\subsubsection{Gás ideal quântico}

Considere um gás de $N$ bósons livres, idênticos e não interagentes em uma caixa cúbica de lado $L$, e suponha que as partículas não possuem graus de liberdade de spin. Nesta circunstância, a Hamiltoniana do sistema será a soma das hamiltonianas de cada bóson, o que implica que a equação de Schrödinger será separável. Logo, a função de onda do sistema será um produto das funções de onda de cada partícula. Assim, para cada indivíduo teremos a equação de Schrödinger independente do tempo com a forma

$$
-\frac{\hbar^{2}}{2 m} \nabla^{2} \psi=E \psi
$$

Impondo condições periódicas de contorno obtemos as energias

$$
E_{n_{x} n_{y} n_{z}}=\frac{\hbar^{2}}{2 m}\left[\left(\frac{2 \pi n_{x}}{L}\right)^{2}+\left(\frac{2 \pi n_{y}}{L}\right)^{2}+\left(\frac{2 \pi n_{z}}{L}\right)^{2}\right]
$$

com os números quânticos $n_{i}$ pertencendo ao conjunto dos números inteiros. Portanto, o estado de uma partícula é determinado pelo conjunto de números quânticos $\vec{n}=$ $\left\{n_{x}, n_{y}, n_{z}\right\}$. Nesta notação a energia possui a forma

$$
E_{\vec{n}}=\frac{\hbar^{2}}{2 m}\left(\frac{2 \pi \vec{n}}{L}\right)^{2}
$$

Na mecânica quântica, todas as partículas de um mesmo tipo são indistinguíveis, o que 
implica que o estado físico do gás é determinando pelo conjunto dos números de ocupação $\left\{N_{\vec{n}}\right\}$ de partículas em um dado estado $\vec{n}$. A energia total do gás em um microestado caracterizado pelo conjunto de números de ocupação $\left\{N_{\vec{n}}\right\}$ é

$$
E=\sum_{\vec{n}} N_{\vec{n}} E_{\vec{n}}
$$

e

$$
N=\sum_{\vec{n}} N_{\vec{n}}
$$

é o número total de partículas, que não esta fixo no ensemble grande canônico.

A soma sobre os microestados (2.18) será então uma soma sobre todos os números de ocupação $N_{\vec{n}}$, que pode ser qualquer no caso bosônico, de tal maneira que

$$
Z_{B}=\sum_{\left\{N_{\vec{n}}=0\right\}}^{\infty} e^{-\beta(E-\mu N)},
$$

onde

$$
\sum_{\left\{N_{\vec{n}}=0\right\}}^{\infty}=\prod_{\vec{n}} \sum_{N_{\vec{n}}=0}^{\infty}
$$

Substituindo (2.29) e (2.30) em (2.31) obtemos

$$
\begin{aligned}
Z_{B} & =\sum_{\left\{N_{\vec{n}}=0\right\}}^{\infty} \exp \left[-\beta \sum_{\vec{n}}\left(N_{\vec{n}} E_{\vec{n}}-\mu N_{\vec{n}}\right)\right] \\
& =\sum_{\left\{N_{\vec{n}}=0\right\}}^{\infty} \prod_{\vec{n}} e^{-\beta N_{\vec{n}}\left(E_{n_{x} n_{y} n_{z}}-\mu\right)}
\end{aligned}
$$

Logo, as somas se fatoram fornecendo

$$
Z_{B}=\prod_{\vec{n}} \sum_{N_{\vec{n}}=0}^{\infty}\left[e^{-\beta E_{\vec{n}}+\beta \mu}\right]^{N_{\vec{n}}} .
$$

Reconhecendo série geométrica temos

$$
Z_{B}=\prod_{n_{x} n_{y} n_{z}} \frac{1}{1-e^{-\beta E_{n_{x} n_{y} n_{z}+\beta \mu}}},
$$

e tomando o logaritmo

$$
\log Z_{B}=-\sum_{n_{x} n_{y} n_{z}} \log \left[1-e^{-\beta E_{n_{x} n_{y} n_{z}}+\beta \mu}\right]
$$


que é a função de partição do gás de Bose no ensemble grande canônico.

Já no caso fermiônico, pelo princípio de exclusão de Pauli, não podemos ter dois férmions idênticos com todos os números quântico iguais. Assim as somas sobre os números de ocupação (2.34 irão apenas até 1. Assim, a função de partição do gás de Fermi assume a forma

$$
\begin{aligned}
Z_{F} & =\prod_{\vec{n}} \sum_{N_{\vec{n}}=0}^{1}\left[e^{-\beta E_{\vec{n}}+\beta \mu}\right]^{N} \\
& =\prod_{n_{x} n_{y} n_{z}}\left[1+e^{-\beta E_{n_{x} n_{y} n_{z}}+\beta \mu}\right],
\end{aligned}
$$

e portanto

$$
\log Z_{F}=\sum_{n_{x} n_{y} n_{z}} \log \left[1+e^{-\beta E_{n_{x} n_{y} n_{z}}+\beta \mu}\right]
$$

que é a função de partição para o gás de Fermi no ensemble grande canônico.

Agora os resultados 2.36 e 2.38 podem ser sintetizados em uma única equação na forma

$$
\log Z_{F / B}= \pm \sum_{n_{x} n_{y} n_{z}} \log \left[1 \pm e^{-\beta E_{n_{x} n_{y} n_{z}}+\beta \mu}\right]
$$

No limite termodinâmico $L \rightarrow \infty$ teremos que a soma irá tender a uma integral. Escrevendo

$$
\frac{2 \pi \Delta n_{x}}{L} \rightarrow d k_{x}
$$

veremos que a soma irá tender á

$$
\log Z_{F / B}= \pm V \int \frac{d^{3} k}{(2 \pi)^{3}} \log \left[1 \pm \exp \left(-\beta \frac{\hbar^{2} \vec{k}^{2}}{2 m}+\beta \mu\right)\right] .
$$

\subsubsection{A matriz densidade}

O estado físico sistema quântico simples pode ser descrito por um estado puro na representação de Schrödinger

$$
|\psi, t\rangle
$$

que possui a evolução temporal dada pela equação de Schrödinger dependente do tempo

$$
H|\psi, t\rangle=i \hbar \frac{\partial}{\partial t}|\psi, t\rangle
$$


ou, de forma equivalente, pelo operador de evolução temporal

$$
|\psi, t\rangle=U\left(t, t^{\prime}\right)\left|\psi, t^{\prime}\right\rangle
$$

Para uma hamiltoniana que não depende explicitamente do tempo, impondo que o estado (2.44) satisfaça a equação de Schrödinger (2.43), vemos que operador de evolução temporal tem a forma

$$
U\left(t, t^{\prime}\right)=e^{\frac{1}{i \hbar} H\left(t-t^{\prime}\right)} .
$$

Da equação 2.44 decorre que conhecendo o estado do sistema em um dado tempo inicial, a evolução temporal deste estado se dará de forma determinística.

Um observável físico, isto é, uma grandeza física que podemos medir, é descrito por um operador hermitiano $O$, cujos autoestados e autovalores correspondentes são as soluções da equação

$$
O|n\rangle=O_{n}|n\rangle
$$

onde $|n\rangle$ é o autoestado e $O_{n}$ é o respectivo autovalor. Dizemos que o autovalor $O_{n}$ é degenerado se existirem dois ou mais autoestados linearmente independentes com o mesmo autovalor $O_{n}$. Caso contrário dizemos que o autovalor é não degenerado.

Ao realizar uma medida do observável $O$ no instante $t$, a probabilidade de obter o valor $O_{n}$ é dada pelo modulo ao quadrado da projeção no autoestado normalizado

$$
P_{n}=|\langle n \mid \psi, t\rangle|^{2}
$$

no caso não degenerado. No caso degenerado será a soma das probabilidades de se obter o autovalor por cada autovetor linearmente independente que possuir o mesmo autovalor. Usando a completeza da base dos autovetores $|n\rangle$ e a normalização do estado, vemos que a probabilidade 2.47) está normalizada a unidade.

O valor médio do observável $O$, ou valor esperado, é construído como uma média ponderada dos valores possíveis para o observável $O_{n}$, tendo como pesos as probabilidades $P_{n}$, como segue

$$
\langle O\rangle=\sum_{n} O_{n} P_{n}=\sum_{n} O_{n}|\langle n \mid \psi, t\rangle|^{2}=\left\langle\psi, t\left|\left(\sum_{n} O_{n}|n\rangle\langle n|\right)\right| \psi, t\right\rangle .
$$

Usando o teorema espectral

$$
O=\sum_{n} O_{n}|n\rangle\langle n|
$$


escrevemos 2.48 como

$$
\langle O\rangle=\langle\psi, t|O| \psi, t\rangle
$$

Se fizermos infinitas cópias do sistema quântico descrito por $|\psi, t\rangle$ e medirmos a grandeza $O$, iremos obter um valor médio dado pela equação (2.50).

Mesmo que na mecânica quântica a evolução temporal do estado se dê de forma determinística, quando medimos um observável físico a natureza probabilística intrínseca da mecânica quântica se manifesta, como pode ser notado em (2.47). Assim o tipo de flutuação decorre da equação (2.47) se deve a natureza quântica do sistema, e mesmo quando conhecermos o estado do sistema exatamente haverá este tipo de flutuação. Já as flutuações térmicas presentes na equação 2.17) decorrem do desconhecimento do estado exato do sistema. Na mecânica estatística quântica ambas as flutuações estão presentes.

O operador matriz densidade é definido para um estado puro como sendo

$$
\rho(t)=|\psi, t\rangle\langle\psi, t|
$$

ou seja, é o projetor no vetor de estado. A equação 2.50 para o valor médio de um observável $O$ em termos do operador matriz densidade é reescrita como

$$
\langle O\rangle=\operatorname{tr}(\rho(t) O)
$$

pois, tomando o traço na base $|n\rangle$ e usando usando a relação de completeza para esta base obtemos

$$
\begin{aligned}
\langle O\rangle & =\operatorname{tr}(\rho(t) O) \\
& =\operatorname{tr}(|\psi, t\rangle\langle\psi, t| O) \\
& =\sum_{n}\langle n \mid \psi, t\rangle\langle\psi, t|O| n\rangle \\
& =\left\langle\psi, t\left|O\left(\sum_{n}|n\rangle\langle n|\right)\right| \psi, t\right\rangle \\
& =\langle\psi, t|O| \psi, t\rangle .
\end{aligned}
$$

A equação de evolução temporal do operador matriz densidade pode ser encontrada 
a partir da evolução do estado como

$$
\begin{aligned}
\frac{\partial}{\partial t} \rho & =\left(\frac{\partial}{\partial t}|\psi, t\rangle\right)\langle\psi, t|+| \psi, t\rangle\left(\frac{\partial}{\partial t}\langle\psi, t|\right) \\
& =\frac{1}{i \hbar}(H|\psi, t\rangle\langle\psi, t|-| \psi, t\rangle\langle\psi, t| H) \\
& =\frac{1}{i \hbar}[H, \rho] .
\end{aligned}
$$

Esta é a equação de Von Neumann para evolução temporal no formalismo da matriz densidade. Alternativamente, podemos descrever a dinâmica da matriz densidade em termos do operador de evolução temporal como

$$
\rho(t)=U\left(t, t^{\prime}\right) \rho\left(t^{\prime}\right) U^{\dagger}\left(t, t^{\prime}\right)
$$

Quando temos um estado puro, podemos descrever o estado físico do sistema tanto pelo vetor de estado, quanto pelo operador matriz densidade. Entretanto, nem sempre conhecemos o estado físico do sistema com exatidão. Em mecânica estatística trabalhamos com sistemas com muitos graus de liberdade, não sendo possível conhecer o estado microscópico do sistema exatamente. Assim, descrevemos as probabilidades do sistema estar em um determinado estado, de modo que a descrição em termos de um único vetor de estado não é adequada aos problemas de mecânica estatística.

Neste contexto, suponha que a probabilidade de se encontrar o sistema em um estado $\left|\psi_{l}, t\right\rangle$ seja $Q_{l}$, com $0 \leq Q_{l} \leq 1$ e

$$
\sum_{l} Q_{l}=1
$$

A probabilidade de obter um valor $O_{n}$ na medida de um observável $O$ no estado $\left|\psi_{l}, t\right\rangle$ é dada por

$$
P_{n, l}=\left|\left\langle n \mid \psi_{l}, t\right\rangle\right|^{2}
$$


Logo, o valor médio do operador $O$ é dado pela média ponderada

$$
\begin{aligned}
\langle O\rangle & =\sum_{l} Q_{l} \sum_{n} P_{n, l} O_{n} \\
& =\sum_{l} Q_{l} \sum_{n}\left\langle\psi_{l}, t \mid n\right\rangle O_{n}\left\langle n \mid \psi_{l}, t\right\rangle \\
& =\sum_{l} Q_{l} \operatorname{tr}\left(\rho_{l} O\right) \\
& =\operatorname{tr}\left[\left(\sum_{l} Q_{l} \rho_{l}\right) O\right] \\
& =\operatorname{tr}(\rho O)
\end{aligned}
$$

de forma que somos levados a generalizar a matriz densidade para uma mistura estatística de estados como

$$
\rho(t)=\sum_{l} Q_{l} \rho_{l}=\sum_{l} Q_{l}\left|\psi_{l}, t\right\rangle\left\langle\psi_{l}, t\right|
$$

Portanto, a matriz densidade de um mistura é a média ponderada das matrizes densidades de estado puro $\rho_{l}=\left|\psi_{l}, t\right\rangle\left\langle\psi_{l}, t\right|$, com os pesos sendo as probabilidades $Q_{l}$ de encontrar o sistema no estado $\left|\psi_{l}, t\right\rangle$. Note que 2.58 nada mais é que uma média estatística sobre médias quânticas.

Como a equação de evolução temporal para a matriz densidade no estado puro 2.55 é linear, para o caso de uma mistura teremos que as expressões

$$
\frac{\partial}{\partial t} \rho=\frac{1}{i \hbar}[H, \rho]
$$

e

$$
\rho(t)=U\left(t, t^{\prime}\right) \rho\left(t^{\prime}\right) U^{\dagger}\left(t, t^{\prime}\right)
$$

são também válidas para uma mistura estatística.

A expressão 2.59 é a forma mais geral que a matriz densidade pode assumir. Deste modo, vemos que o traço da matriz densidade é sempre normalizado a unidade

$$
\operatorname{tr} \rho(t)=\sum_{l} Q_{l} \sum_{n}\left|\left\langle n \mid \psi_{l}, t\right\rangle\right|^{2}=\sum_{l} Q_{l}=1
$$

onde usamos a completeza da base e o fato que as probabilidades $Q_{l}$ são normalizadas. Em um estado puro a matriz densidade possui a forma (2.51) de modo que teremos $\rho^{2}=\rho$, e portanto $\operatorname{tr} \rho^{2}=1$. Entretanto, em uma mistura estatística temos que $\operatorname{tr} \rho^{2}<1$, de modo que o traço de $\rho^{2}$ é o critério para determinar se temos uma mistura estatística ou um 
estado puro.

Vamos mostrar isso no caso em que os estados $\left|\psi_{l}\right\rangle$ são autoestados de algum observável físico, que é o caso da matriz densidade para sistemas em equilíbrio térmico, como veremos adiante. Neste caso a matriz densidade assume a forma

$$
\rho=\sum_{n} Q_{n}|n\rangle\langle n|
$$

de onde segue que

$$
\operatorname{tr} \rho=\sum_{n} Q_{n}=1
$$

e

$$
\operatorname{tr} \rho^{2}=\sum_{n} Q_{n}^{2}
$$

Para um estado puro, teremos $Q_{m}=1$ para algum $m$ e $Q_{n}=0$ para $n \neq m$. Logo para um estado puro $\rho^{2}=\rho$ e, portanto, $\operatorname{tr} \rho^{2}=1$. Já quando temos uma mistura estatística, $0 \leq Q_{n}<1$ e, para todo $Q_{n}$ não nulo, temos que $Q_{n}^{2}<Q_{n}$, e certamente teremos mais de um $Q_{n}$ não nulo, caso contrario teríamos um estado puro. Logo, quando temos uma mistura $\operatorname{tr} \rho^{2}<1$. Como a evolução temporal na mecânica quântica é unitária, a equação 2.61 implica que o traço de $\rho^{2}$ não muda no tempo. Portanto, vemos que um estado puro não pode evoluir para uma mistura do mesmo modo que uma mistura não pode evoluir para um estado puro.

Considere um sistema em equilíbrio térmico descrito pela hamiltoniana $H$. Suponha que temos um operador número conservado $N$, isto é

$$
[H, N]=0 .
$$

Como esses operadores são compatíveis, temos que o espectro de ambos pode ser descrito em termos de um conjunto de autoestados comuns, do modo que

$$
H|n\rangle=E_{n}|n\rangle
$$

$\mathrm{e}$

$$
N|n\rangle=N_{n}|n\rangle
$$

Conforme vimos na seção 2.1.2 [veja a equação (2.17]), no equilíbrio térmico a uma temperatura $T$ e um potencial químico $\mu$, temos que a probabilidade de se encontrar o sistema em um estado 
é proporcional a

$$
e^{-\beta\left(E_{n}-\mu N_{n}\right)}
$$

conforme a equação (2.17). Então, a matriz densidade para este sistema pode ser escrita como

$$
\begin{aligned}
\rho & =Z^{-1} \sum_{n} e^{-\beta\left(E_{n}-\mu N_{n}\right)}|n\rangle\langle n| \\
& =Z^{-1} \sum_{n} \sum_{k} \frac{\left[-\beta\left(E_{n}-\mu N_{n}\right)\right]^{k}}{k !}|n\rangle\langle n|
\end{aligned}
$$

onde $Z$ é uma normalização que fixa $\operatorname{tr} \rho=1$. Usando o teorema espectral, vemos que podemos escrever

$$
(H-\mu N)=\sum_{n}|n\rangle\left(E_{n}-\mu N_{n}\right)\langle n|
$$

e multiplicando membro a membro por $(H-\mu N)^{k-1}$ obtemos que

$$
(H-\mu N)^{k}=\sum_{n}|n\rangle\left(E_{n}-\mu N_{n}\right)^{k}\langle n|
$$

Substituindo este resultado em 2.71) escrevemos $\rho$ como

$$
\rho=Z^{-1} e^{-\beta(H-\mu N)}
$$

onde

$$
Z=\operatorname{tr} e^{-\beta(H-\mu N)}
$$

é a função de partição. O resultado 2.74 é a base para os cálculo de valores esperados térmicos que faremos neste trabalho.

\subsection{Função de partição no formalismo funcional}

Nesta seção faremos uma breve introdução aos métodos funcionais em teorias de campos. Escreveremos a função de partição no formalismo funcional para o campo escalar neutro. Em seguida estenderemos os resultados para o campo de Dirac. Iremos notar que no formalismo do tempo imaginário a integração funcional se dá sobre modos periódicos dos campos bosônico e anti-periódicos do campo fermiônico. Por último, iremos analisar o caso dos campos de calibre. Veremos que não é possível definir a teoria de perturbação usual quando as simetrias de calibre estão presentes. Para solucionar este problema, revisaremos os argumentos de Faddeev-Popov [FP67] em um contexto geral. Em seguida aplicaremos o raciocínio no contexto do campo de Maxwell em uma classe de calibres 
tipo Lorentz, na qual o calibre de Feynman, que é o calibre escolhido neste trabalho, está incluso.

\subsubsection{O formalismo da integração funcional}

Com intuito de ilustrar conexão entre os formalismos de operadores e o formalismo da integração funcional, calcularemos amplitudes de transição para bósons no formalismo funcional. As amplitudes de transição são de fundamental importância no cálculo da função de partição, que é o ingrediente básico do nosso estudo das propriedades térmicas dos sistemas quânticos.

Considere um campo escalar $\phi(\vec{x}, t)$, cuja a densidade lagrangiana possui a forma

$$
\mathcal{L}=\frac{1}{2} \partial_{\mu} \phi \partial^{\mu} \phi-\frac{m^{2}}{2} \phi^{2}-V(\phi)
$$

e a equação de movimento para este campo é a equação de Klein-Gordon

$$
\partial_{\mu} \partial^{\mu} \phi+m^{2} \phi+\frac{\partial V}{\partial \phi}=0
$$

O momento canonicamente conjugado ao campos $\Pi(\vec{x}, t)$, é obtido de maneira usual

$$
\Pi(\vec{x}, t)=\frac{\partial \mathcal{L}}{\partial\left(\partial_{0} \phi\right)}=\partial^{0} \phi
$$

que implica em uma hamiltoniana na forma

$$
H=\int d^{d-1} x \mathcal{H}=\int d^{d-1} x\left[\frac{1}{2} \Pi^{2}+\frac{1}{2}(\vec{\nabla} \phi)^{2}+\frac{1}{2} m^{2} \phi^{2}+V(\phi)\right],
$$

onde $d$ é a dimensionalidade do espaço-tempo. Para sistema contínuos o parêntese de Poisson possuem a forma

$$
\{A(t), B(t)\}=\int d^{d-1} y\left[\frac{\delta A}{\delta \phi(y, t)} \frac{\delta B}{\delta \Pi(y, t)}-\frac{\delta A}{\delta \Pi(y, t)} \frac{\delta B}{\delta \phi(y, t)}\right]
$$

que implica nas equações de Hamilton

$$
\begin{aligned}
& \partial_{0} \phi(\vec{x}, t)=\{\phi(\vec{x}, t), H\}=\Pi(\vec{x}, t) \\
& \partial_{0} \Pi(\vec{x}, t)=\{\Pi(\vec{x}, t), H\}=\nabla^{2} \phi(\vec{x}, t)-m^{2} \phi(\vec{x}, t)-\frac{\partial V}{\partial \phi}
\end{aligned}
$$

que se equivalem a equação de Klein-Gordon.

A canônica quantização é feita promovendo o campo e o momento conjugado à operadores. Denotamos $\hat{\phi}(\vec{x}, t)$ como o campo na representação de Heisenberg no instante t 
e $\hat{\Pi}(\vec{x}, t)$ seu momento canonicamente conjugado. Eles satisfazem regras de comutação canônicas em tempos iguais

$$
\begin{aligned}
{[\hat{\phi}(\vec{x}, t), \hat{\Pi}(\vec{y}, t)] } & =i \delta(\vec{x}-\vec{y}) \\
{[\hat{\phi}(\vec{x}, t), \hat{\phi}(\vec{y}, t)] } & =[\hat{\Pi}(\vec{x}, t), \hat{\Pi}(\vec{y}, t)]=0 .
\end{aligned}
$$

A dinâmica dos operadores na descrição de Heisenberg é dada em termos do operador de evolução temporal por

$$
\begin{aligned}
\hat{\phi}(\vec{x}, t) & =U^{\dagger}(t) \hat{\phi}(\vec{x}, 0) U(t) \\
\hat{\Pi}(\vec{x}, t) & =U^{\dagger}(t) \hat{\Pi}(\vec{x}, 0) U(t),
\end{aligned}
$$

e como a hamiltoniana não depende explicitamente do tempo o operador de evolução temporal possui a forma

$$
U(t)=e^{-i H t}
$$

As equações de evolução 2.83 implicam nas equações de Heisenberg

$$
\begin{aligned}
\partial_{0} \phi & =i[H, \phi]=\Pi \\
\partial_{0} \Pi=i[H, \Pi] & =\nabla^{2} \phi-m^{2} \phi-\frac{\partial V}{\partial \phi},
\end{aligned}
$$

que são idênticas na forma as equações de Hamilton (2.81). Logo, na representação de Heisenberg, os operadores de campo satisfazem a equação de Klein-Gordon.

No tempo $t=0$ as representações de Heisenberg e de Schrödinger coincidem. Assim denotamos o campo na representação de Schrödinger por $\hat{\phi}(\vec{x}, 0)$ e seu momento conjugado por $\hat{\Pi}(\vec{x}, 0)$. Definimos os "kets" $|\phi\rangle$ e $|\Pi\rangle$ como sendo autoestados do campo e do seu momento conjugado na representação de Schrödinger, respectivamente,

$$
\begin{aligned}
\hat{\phi}(\vec{x}, 0)|\phi\rangle & =\phi(\vec{x})|\phi\rangle, \\
\hat{\Pi}(\vec{x}, 0)|\Pi\rangle & =\Pi(\vec{x})|\Pi\rangle,
\end{aligned}
$$

onde $\phi(\vec{x})$ e $\Pi(\vec{x})$ são os respectivos autovalores. Multiplicando 2.86 membro a membro por $U^{\dagger}(t, 0)$ e usando a unitariedade do operador de evolução temporal obtemos

$$
U^{\dagger}(t, 0) \hat{\phi}(\vec{x}, 0) U(t, 0) U^{\dagger}(t, 0)|\phi\rangle=\hat{\phi}(\vec{x}, t)|\phi ; t\rangle=\phi(\vec{x})|\phi ; t\rangle
$$

ou seja, os autovalores do campo e de seu momento conjugado não variam no tempo na descrição de Heisenberg (note que o autovetor no tempo $t$ é dado por $U^{\dagger}(t, 0)|\phi\rangle$ ). 
Os "kets" $|\phi\rangle$ e $|\Pi\rangle$ obedecem as relações de completeza

$$
\begin{aligned}
& \int \mathcal{D} \phi|\phi\rangle\langle\phi|=1, \\
& \int \mathcal{D} \Pi|\Pi\rangle\langle\Pi|=1 .
\end{aligned}
$$

As integrais acima são integrais funcionais cujo domínio é conjunto de todos dos autovalores possíveis. A forma explícita destas integrais está expressa no apêndice A. Os autoestados dos campos e do momento conjugado satisfazem relações de ortogonalidade

$$
\begin{aligned}
\left\langle\phi_{a} \mid \phi_{b}\right\rangle & =\delta\left[\phi_{a}-\phi_{b}\right], \\
\left\langle\Pi_{a} \mid \Pi_{b}\right\rangle & =\delta\left[\Pi_{a}-\Pi_{b}\right],
\end{aligned}
$$

que nos diz apenas que autovetores com autovalores distintos são ortogonais. Em analogia com a representação de Fourier da delta de Dirac

$$
(2 \pi) \delta(x-y)=\int d p e^{i p(x-y)}
$$

o funcional $\delta$ é definido no espaço das funções como uma integral funcional

$$
\delta\left[\phi_{a}-\phi_{b}\right]=\int \mathcal{D} \xi \exp \left\{i \int d^{d-1} x \xi(\vec{x})\left[\phi_{a}(\vec{x})-\phi_{b}(\vec{x})\right]\right\} .
$$

Suponha que no instante inicial $t=0$ o sistema se encontre no estado $\left|\phi_{a}\right\rangle$. Então no instante de tempo $t=T$ ele se encontrará no estado $e^{-i H T}\left|\phi_{a}\right\rangle$. Neste instante de tempo a amplitude de transição para um estado $\left|\phi_{b}\right\rangle$ será dada por

$$
\left\langle\phi_{b}\left|e^{-i H T}\right| \phi_{a}\right\rangle
$$

Vamos dividir o intervalo de tempo $T$ em $N$ intervalos de duração $\epsilon$. Assim $T=N \epsilon$ e definimos $t_{n}=n \epsilon$ para $n=0,1, \ldots, N$. Inserindo as resoluções da unidades 2.89) e 2.90) em 2.95 obtemos

$$
\begin{aligned}
\left\langle\phi_{b}\left|e^{-i H T}\right| \phi_{a}\right\rangle & =\prod_{n=1}^{N} \int \mathcal{D} \phi_{n} \mathcal{D} \Pi_{n}\left\langle\phi_{b} \mid \Pi_{N}\right\rangle\left\langle\Pi_{N}\left|e^{-i H \epsilon}\right| \phi_{N}\right\rangle\left\langle\phi_{N} \mid \Pi_{N-1}\right\rangle \\
& \times\left\langle\Pi_{N-1}\left|e^{-i H \epsilon}\right| \phi_{N-1}\right\rangle \ldots\left|\Pi_{2}\right\rangle\left\langle\Pi_{2}\left|e^{-i H \epsilon}\right| \phi_{2}\right\rangle \\
& \times\left\langle\phi_{2} \mid \Pi_{1}\right\rangle\left\langle\Pi_{1}\left|e^{-i H \epsilon}\right| \phi_{1}\right\rangle\left\langle\phi_{1} \mid \phi_{a}\right\rangle .
\end{aligned}
$$


Na mecânica quântica temos que

$$
\langle x \mid p\rangle=e^{i p x}
$$

Portanto, estendendo para a teoria de campos teremos

$$
\langle\phi \mid \Pi\rangle=e^{i \int d^{d-1} x \Pi(\vec{x}) \phi(\vec{x})} .
$$

Vamos calcular o elemento de matriz $\left\langle\Pi_{n}\left|e^{-i H \epsilon}\right| \phi_{n}\right\rangle$ no limite de $\epsilon$ infinitesimal, supondo que os operadores $\hat{\Pi}$ estão sempre a esquerda dos operadores $\hat{\phi}$ na hamiltoniana, de modo que escrevemos

$$
\begin{aligned}
\left\langle\Pi_{n}\left|e^{-i H \epsilon}\right| \phi_{n}\right\rangle & \approx\left\langle\Pi_{n}|(1-i H \epsilon)| \phi_{n}\right\rangle \\
& =\left\langle\Pi_{n}\left|\left[1-i \epsilon \int d^{d-1} x \mathcal{H}(\hat{\phi}(\vec{x}), \hat{\Pi}(\vec{x}))\right]\right| \phi_{n}\right\rangle \\
& =\left\langle\Pi_{n} \mid \phi_{n}\right\rangle\left[1-i \epsilon \int d^{d-1} x \mathcal{H}\left(\phi_{n}(\vec{x}), \Pi_{n}(\vec{x})\right)\right]
\end{aligned}
$$

onde $\mathcal{H}\left(\phi_{n}(\vec{x}), \Pi_{n}(\vec{x})\right)$ é a densidade Hamiltoniana substituindo os operadores pelos autovalores, ou seja, é a Hamiltoniana clássica. Para um tratamento mais rigoroso para a questão da ordenação veja [Das93]. Portanto, substituindo (2.98) em (2.99) obtemos

$$
\left\langle\Pi_{n}\left|e^{-i H \epsilon}\right| \phi_{n}\right\rangle \approx e^{-i \int d^{d-1} x\left[\Pi_{n}(\vec{x}) \phi_{n}(\vec{x})+\epsilon \mathcal{H}\left(\phi_{n}(\vec{x}), \Pi_{n}(\vec{x})\right)\right]},
$$

em primeira ordem em $\epsilon$.

Substituindo 2.98 e 2.100 em 2.96) obtemos

$$
\begin{aligned}
\left\langle\phi_{b}\left|e^{-i H t}\right| \phi_{a}\right\rangle & =\prod_{n=1}^{N} \int \mathcal{D} \Pi_{n} \mathcal{D} \phi_{n} \delta\left[\phi_{1}-\phi_{a}\right] \\
& \times \exp \left\{i \epsilon \sum_{m=1}^{N} \int d^{d-1} x\left[\Pi_{m}\left(\frac{\phi_{m+1}-\phi_{m}}{\epsilon}\right)-\mathcal{H}\left(\Pi_{m}, \phi_{m}\right)\right]\right\},
\end{aligned}
$$

com $\phi_{N+1}=\phi_{b}$. Como os autovalores possíveis do operador campo e seu momento conjugado não variam no tempo na representação de Heisenberg, é consistente pensar na correspondência

$$
\phi_{n}(\vec{x}) \rightarrow \phi\left(\vec{x}, t_{n}\right),
$$

de forma que podemos pensar em 2.101 como uma soma sobre todas as possíveis configurações de campo para os tempos intermediários $0<t_{n}<T$. Assim, tomando o limite $N \rightarrow \infty$ ou $\epsilon \rightarrow 0$ podemos fazer uma correspondência entre o índice discreto $n$ com o 
tempo contínuo, de forma que 2.101) será dada por

$$
\left\langle\phi_{b}\left|e^{-i H T}\right| \phi_{a}\right\rangle=\int \mathcal{D} \Pi \int_{\phi_{a}}^{\phi_{b}} \mathcal{D} \phi \exp \left[i \int_{0}^{T} d t \int d^{d-1} x\left(\Pi \partial_{0} \phi-\mathcal{H}\right)\right],
$$

onde $\int \mathcal{D} \phi=\prod_{n=1}^{N} \int \mathcal{D} \phi_{n}$. Esta expressão acima é a amplitude de transição para bósons no formalismo da integração funcional. A definição mais precisa desta expressão é obtida na rede (veja apêndice A), como um limite na forma

$$
\begin{aligned}
\left\langle\phi_{b}\left|e^{-i H T}\right| \phi_{a}\right\rangle & =\lim _{\Delta v_{l} \rightarrow 0} \lim _{\Delta t_{n} \rightarrow 0} \prod_{n=1}^{N} \prod_{l} \int_{-\infty}^{\infty} d \Pi_{i}\left(\vec{x}_{l}\right) d \phi_{i}\left(\vec{x}_{l}\right) \\
& \times \exp \left\{i \sum _ { m } \Delta t _ { m } \sum _ { j } \Delta v _ { j } \left[\Pi_{m}\left(\vec{x}_{j}\right) \frac{\phi_{m+1}\left(\vec{x}_{j}\right)-\phi_{m}\left(\vec{x}_{j}\right)}{\Delta t_{m}}\right.\right. \\
& \left.\left.-\mathcal{H}\left(\phi_{m}\left(\vec{x}_{j}\right), \Pi_{m}\left(\vec{x}_{j}\right)\right)\right]\right\} .
\end{aligned}
$$

Substituindo a hamiltoniana 2.79 em 2.103$)$ obtemos

$$
\begin{aligned}
\left\langle\phi_{b}\left|e^{-i H T}\right| \phi_{a}\right\rangle & =\int \mathcal{D} \Pi \int_{\phi_{a}}^{\phi_{b}} \mathcal{D} \phi \exp \left\{i \int _ { 0 } ^ { T } d t \int d ^ { d - 1 } x \left[\Pi \partial_{0} \phi-\frac{1}{2} \Pi^{2}\right.\right. \\
& \left.\left.-\frac{1}{2}(\vec{\nabla} \phi)^{2}-\frac{m^{2}}{2} \phi^{2}-V(\phi)\right]\right\}
\end{aligned}
$$

As integrais em П são gaussianas e para resolve-las vamos completar o quadrado reescrevendo

$$
\begin{aligned}
\left\langle\phi_{b}\left|e^{-i H T}\right| \phi_{a}\right\rangle & =\int \mathcal{D} \Pi \int_{\phi_{a}}^{\phi_{b}} \mathcal{D} \phi \exp \left\{i \int _ { 0 } ^ { T } d t \int d ^ { d - 1 } x \left[-\frac{1}{2}\left(\Pi-\partial_{0} \phi\right)^{2}\right.\right. \\
& \left.\left.+\frac{1}{2}\left(\partial_{0} \phi\right)^{2}-\frac{1}{2}(\vec{\nabla} \phi)^{2}-\frac{m^{2}}{2} \phi^{2}-V(\phi)\right]\right\}
\end{aligned}
$$

e reconhecendo a densidade lagrangiana 2.76 escrevemos

$$
\left\langle\phi_{b}\left|e^{-i H T}\right| \phi_{a}\right\rangle=\int \mathcal{D} \Pi \int_{\phi_{a}}^{\phi_{b}} \mathcal{D} \phi \exp \left\{i \int_{0}^{T} d t \int d^{d-1} x\left[-\frac{1}{2}\left(\Pi-\partial_{0} \phi\right)^{2}+\mathcal{L}\right]\right\} .
$$

Fazendo uma mudança de variáveis na integração em $\Pi$

$$
\Pi \rightarrow \Pi^{\prime}=\Pi-\partial_{0} \phi
$$

vemos que a gaussiana integral no momento canônico ПI se fatora. Escrevendo esta gaus- 
siana na rede, temos

$$
\begin{aligned}
\int \mathcal{D} \Pi \exp \left(-i \int_{0}^{T} d t \int d^{d-1} x \frac{1}{2} \Pi^{2}\right) & =\prod_{n, l} \int_{-\infty}^{\infty} d \Pi_{n}\left(\vec{x}_{l}\right) \exp \left[-i \sum_{m} \Delta t_{m} \sum_{j} \Delta v_{j} \frac{1}{2} \Pi_{m}\left(\vec{x}_{j}\right)^{2}\right] \\
& =\lim _{N, L \rightarrow \infty}\left[\frac{2 \pi i}{\Delta t \Delta v}\right]^{N L}
\end{aligned}
$$

com os vínculos $L \Delta v=V$ e $N \Delta t=T$. Convencionalmente, incorporamos este fator na medida de integração, de modo que teremos

$$
\left\langle\phi_{b}\left|e^{-i H T}\right| \phi_{a}\right\rangle=\int_{\phi_{a}}^{\phi_{b}} \mathcal{D}[\phi] e^{i S[\phi]}
$$

onde $S[\phi]$ é a ação clássica e

$$
\int \mathcal{D}[\phi]=\prod_{n=1}^{N} \prod_{l=1}^{L} \frac{2 \pi i}{\Delta t \Delta v} \int_{-\infty}^{\infty} d \phi_{n}\left(\vec{x}_{l}\right) .
$$

A equação 2.110 é a amplitude de transição para bósons no formalismo funcional. Ela possui a forma de uma soma de todas as todas as configurações possíveis campo $\phi$ entre os tempos 0 e $T$, com a condição de contorno $\phi(0)=\phi_{a}$ e $\phi(T)=\phi_{b}$, tendo como peso a exponencial da ação.

Uma importante generalização de 2.110 para correlações entre campos pode ser obtida de maneira simples. Considere a correlação de dois pontos

$$
\left\langle\phi_{b}\left|e^{-i H T} T\left[\hat{\phi}\left(\vec{x}_{1}, t_{1}\right) \hat{\phi}\left(\vec{x}_{2}, t_{2}\right)\right]\right| \phi_{a}\right\rangle
$$

onde $T[\cdot]$ é o produto ordenando no tempo. No caso em que $t_{1}<t_{2}$, usando a equação 2.83 temos

$$
\left\langle\phi_{b}\left|e^{-i H T} T\left[\hat{\phi}\left(\vec{x}_{1}, t_{1}\right) \hat{\phi}\left(\vec{x}_{2}, t_{2}\right)\right]\right| \phi_{a}\right\rangle=\left\langle\phi_{b}\left|e^{-i H\left(T-t_{1}\right)} \hat{\phi}\left(\vec{x}_{1}, 0\right) e^{-i H\left(t_{1}-t_{2}\right)} \hat{\phi}\left(\vec{x}_{2}, 0\right) e^{-i H t_{2}}\right| \phi_{a}\right\rangle .
$$

Portanto, podemos repetir os mesmos passos que levaram à equação 2.110 para obter

$$
\left\langle\phi_{b}\left|e^{-i H T} T\left[\hat{\phi}\left(\vec{x}_{1}, t_{1}\right) \hat{\phi}\left(\vec{x}_{2}, t_{2}\right)\right]\right| \phi_{a}\right\rangle=\int_{\phi_{a}}^{\phi_{b}} \mathcal{D}[\phi] e^{i S[\phi]} \phi\left(\vec{x}_{1}, t_{1}\right) \phi\left(\vec{x}_{2}, t_{2}\right) .
$$

Como expressão uma expressão análoga a 2.113 pode ser escrita para um produto de número arbitrário de operadores de campo, temos para qualquer função do campo $\hat{\phi}$ vale

$$
\left\langle\phi_{b}\left|e^{-i H T} T[O(\hat{\phi})]\right| \phi_{a}\right\rangle=\int_{\phi_{a}}^{\phi_{b}} \mathcal{D}[\phi] e^{i S[\phi]} O(\phi) .
$$




\subsubsection{A função de partição para o campo escalar real livre}

Conforme vimos na seção 2.1.4 para um sistema em equilíbrio térmico a temperatura $T$, com um potencial químico $\mu$ relativo ao operador conservado

$$
N=\int d^{d-1} x \mathcal{N}
$$

temos que a matriz densidade possui a forma

$$
\rho=Z^{-1} e^{-\beta(H-\mu N)},
$$

Assim, da normalização $\operatorname{tr} \rho=1$, temos que $Z$ é dado por:

$$
Z=\operatorname{Tr} e^{-\beta(H-\mu N)}
$$

que é a função de partição do ensemble grande canônico, ou grande função de partição. Escrevendo o traço na base dos auto estados do operador de campo $\left|\phi_{a}\right\rangle$ temos que a função de partição é reescrita como

$$
Z=\int \mathcal{D} \phi_{a}\left\langle\phi_{a}\left|e^{-\beta(H-\mu N)}\right| \phi_{a}\right\rangle
$$

Assim, fazendo $i d t \rightarrow d \tau, i T \rightarrow \beta$ e $H \rightarrow K=H-\mu N$ em 2.103 e fazendo os mesmo passos que levaram até a expressão 2.110 obtemos uma expressão para a função de partição no formalismo da integração funcional na forma

$$
Z=\int_{\text {periódico }} \mathcal{D}[\phi] \exp \left\{\int_{0}^{\beta} d \tau \int d^{d-1} x\left[\mathcal{L}_{e}+\mu \mathcal{N}\right]\right\},
$$

onde

$$
N=\int d^{d-1} x \mathcal{N}
$$

e $\mathcal{L}_{e}$ é a euclideana, isto é,

$$
\mathcal{L}_{e}=\mathcal{L}\left(x_{0}=-i \tau\right)
$$

Como $|\phi\rangle$ e $-|\phi\rangle$ representam o mesmo estado físico, poderíamos pensar em uma soma sobre estados anti-periódicos para o cálculo do traço da matriz densidade 2.119. Para sanar esta questão, vamos estudar o comportamento das funções de correlação ou funções de Green térmicas. As função de Green térmica de dois pontos, é definida como a média térmica

$$
G_{\beta}\left(\vec{x}, \tau_{x} ; \vec{y}, \tau_{y}\right)=\left\langle T_{\tau} \phi\left(\vec{x}, \tau_{x}\right) \phi\left(\vec{y}, \tau_{y}\right)\right\rangle_{\text {ensemble }}
$$


onde $T_{\tau}$ é o operador de ordenação temporal para o tempo imaginário, definido como,

$$
T_{\tau} \phi\left(\vec{y}, \tau_{y}\right) \phi\left(\vec{x}, \tau_{x}\right)=\theta\left(\tau_{y}-\tau_{x}\right) \phi\left(\vec{y}, \tau_{y}\right) \phi\left(\vec{x}, \tau_{x}\right) \pm \theta\left(\tau_{x}-\tau_{y}\right) \phi\left(\vec{x}, \tau_{x}\right) \phi\left(\vec{y}, \tau_{y}\right)
$$

com o sinal positivo para bósons e negativo para férminons.

Usando a definição do valor esperado em um ensemble (2.58), escrevemos a função de Green térmica para $\tau$ no intervalo $(0, \beta)$ como

$$
\begin{aligned}
G_{\beta}(\vec{x}, \tau ; \vec{y}, 0) & =\left\langle T_{\tau} \phi(\vec{y}, 0) \phi(\vec{x}, \tau)\right\rangle \\
& =\operatorname{Tr}(\rho \phi(\vec{x}, \tau) \phi(\vec{y}, 0)) .
\end{aligned}
$$

Usando a ciclicidade do traço, temos

$$
\begin{aligned}
G_{\beta}(\vec{x}, \tau ; \vec{y}, 0) & =\operatorname{Tr}(\phi(\vec{y}, 0) \rho \phi(\vec{x}, \tau)) \\
& =\operatorname{Tr}\left(\rho \rho^{-1} \phi(\vec{y}, 0) \rho \phi(\vec{x}, \tau)\right) .
\end{aligned}
$$

No formalismo do tempo imaginário, fazemos as correspondências

$$
\begin{aligned}
t & \rightarrow-i \beta \\
H & \rightarrow K=H-\mu N \\
U=e^{-i H t} & \rightarrow e^{-\beta(H-\mu N)} .
\end{aligned}
$$

Desta forma, a evolução do estados no formalismo do tempo imaginário é ditada pelo operador matriz densidade

$$
\phi(\vec{x}, \beta)=\rho^{-1} \phi(\vec{x}, 0) \rho
$$

na descrição de Heisenberg. Substituindo 2.127) em (2.125), vemos que as funções de Green térmicas satisfazem relações de periodicidade

$$
\begin{aligned}
G_{\beta}(\vec{x}, \tau ; \vec{y}, 0) & =\operatorname{Tr}(\rho \phi(\vec{y}, \beta) \phi(\vec{x}, \tau)) \\
& = \pm \operatorname{Tr}\left(\rho T_{\tau} \phi(\vec{x}, \tau) \phi(\vec{y}, \beta)\right) \\
& = \pm G_{\beta}(\vec{x}, \tau ; \vec{y}, \beta),
\end{aligned}
$$

onde o sinal + corresponde ao caso bosônico enquanto o sinal - corresponde ao caso fermiônico.

Concluímos então que para o caso bosônico as funções de Green térmicas são periódicas enquanto no caso fermiônico as funções de Green térmicas são a anti-periódicas. As 
funções de Green térmicas são elementos de matriz do operador matriz densidade. Assim 2.128 implica que os estados que possuem a evolução ditada por 2.127) são periódicos no caso bosônico e anti-periódicos no caso fermiônico.

\subsubsection{Função de partição para férmions}

A descrição dos campos de spin 1/2 é dada pela lagrangiana de Dirac

$$
\mathcal{L}_{D}=\bar{\psi}\left(i \gamma^{\mu} \partial_{\mu}-m\right) \psi
$$

com $\bar{\psi}=\psi^{\dagger} \gamma^{0}$. A equação de Euler-Lagrange para $\bar{\psi}$ fornece a equação de Dirac

$$
\left(i \gamma^{\mu} \partial_{\mu}-m\right) \psi=0
$$

Atuando o operador $\left(i \gamma^{\mu} \partial_{\mu}+m\right)$ e impondo consisténcia com a equação de Klein-Gordon, vemos que as matrizes de Dirac satisfaz a álgebra de Clifford

$$
\left\{\gamma^{\mu}, \gamma^{\nu}\right\}=2 \eta^{\mu \nu}
$$

A lagrangiana de Dirac é $U(1)$ global simétrica, pois fazendo

$$
\psi \rightarrow \psi^{\prime}=e^{i \alpha} \psi
$$

a lagrangiana é invariante. Pelo teorema de Noether termos uma corrente conservada associada a essa simetria. Para obter essa corrente vamos escrever a equação de EulerLagrange para $\bar{\psi}$

$$
-i \partial_{\mu} \bar{\psi} \gamma^{\mu}-m \bar{\psi}=0
$$

Multiplicando (2.130) a esquerda por $\bar{\psi}$ e $(2.133)$ a direita por $\psi$ e subtraindo $(2.133)$ de 2.130, obtemos

$$
\partial_{\mu} j^{\mu}=0
$$

com

$$
j^{\mu}=\bar{\psi} \gamma^{\mu} \psi
$$

A conservação desta corrente implica implica que teremos um número quântico conservado

$$
N=\int d^{d-1} x j^{0}=\int d^{d-1} x \bar{\psi} \gamma^{0} \psi=\int d^{d-1} x \psi^{\dagger} \psi
$$


onde usamos que $\left(\gamma^{0}\right)^{2}=1$, segundo a álgebra de Clifford.

O momento canonicamente conjugado ao campo $\psi$ será

$$
\Pi=\frac{\partial \mathcal{L}_{D}}{\partial \partial_{0} \psi}=i \bar{\psi} \gamma^{0}=i \psi^{\dagger}
$$

Assim no formalismo hamiltoniano, trataremos $\psi$ e $\Pi=i \psi^{\dagger}$ como variáveis dinâmicas independentes. A hamiltoniana possui então a forma

$$
\mathcal{H}=\Pi \partial_{0} \psi-\mathcal{L}_{D}=-\bar{\psi}(i \vec{\gamma} \cdot \vec{\partial}-m) \psi
$$

e a função de partiação terá a forma

$$
\begin{aligned}
Z & =\operatorname{tr} e^{-\beta(H-\mu N)} \\
& =\operatorname{tr} e^{-\beta \int d^{d-1} x\left(\mathcal{H}-\mu \bar{\psi} \gamma^{0} \psi\right)} .
\end{aligned}
$$

Segundo o teorema spin estatística, temos que o campo de Dirac satisfaz regras de quantização conônicas na forma de anti-comutadores em tempos iguais

$$
\begin{aligned}
& \left\{\psi_{\alpha}(\vec{x}), \psi_{\beta}^{\dagger}(\vec{y})\right\}=\delta(\vec{x}-\vec{y}) \delta_{\alpha \beta} \\
& \left\{\psi_{\alpha}(\vec{x}), \psi_{\beta}(\vec{y})\right\}=\left\{\psi_{\alpha}^{\dagger}(\vec{x}), \psi_{\beta}^{\dagger}(\vec{y})\right\}=0
\end{aligned}
$$

com isso, no limite clássico, vemos que o campo de Dirac é descrito em termos de variáveis de Grassmman. Assim, repitindo os passos que levaram a função de partição para bósons no formalismo da integração funcional 2.120) e usando os resultados do apêndice B [veja a equação (B.56] ] obtemos a seginte forma para a função de partição do campo de Dirac

$$
Z=\int_{\text {anti-per. }} \mathcal{D}(\bar{\psi}, \psi) e^{-\int_{0}^{\beta} d^{d} x\left[\mathcal{H}-\mu \bar{\psi} \gamma^{0} \psi+\psi^{\dagger} \partial_{\tau} \psi\right]},
$$

com a notação

$$
\int_{0}^{\beta} d^{d} x=\int_{0}^{\beta} d \tau \int d^{d-1} x
$$

escrevendo $\partial_{\tau}=-i \partial_{0}$, obtemos

$$
Z=\int \mathcal{D}(\bar{\psi}, \psi) e^{\int_{0}^{\beta} d^{d} x \bar{\psi}\left(i \gamma^{\mu} \partial_{\mu}-m+\mu \gamma^{0}\right) \psi}
$$

sobre condições anti-periódicas de contorno [veja 2.128 e B.56]

$$
\begin{aligned}
\psi(\vec{x}, \beta) & =-\psi(\vec{x}, 0) \\
\psi^{\dagger}(\vec{x}, \beta) & =-\psi^{\dagger}(\vec{x}, 0) .
\end{aligned}
$$


Com esta condições de contorno, o campo de Dirac assumi uma representação de Fourier do tipo

$$
\psi(\vec{x}, \tau)=\frac{1}{\sqrt{V}} \sum_{n, \vec{p}} e^{-i\left(\tau \omega_{n}^{F}+\vec{x} \cdot \vec{p}\right)} \tilde{\psi}_{n}(\vec{p})
$$

onde $\omega_{n}^{F}=(2 n+1) \pi T$. Usando a notação $x_{0}=-i \tau$ e $p_{0}=i \omega_{n}^{F}$, reescrevendo

$$
\psi(\vec{x}, \tau)=\frac{1}{\sqrt{V}} \sum_{n, \vec{p}} e^{-i x^{\mu} p_{\mu}} \tilde{\psi}_{n}(\vec{p})
$$

De maneira análoga teremos

$$
\psi^{\dagger}(\vec{x}, \tau)=\frac{1}{\sqrt{V}} \sum_{n, \vec{p}} e^{i x^{\mu} p_{\mu}} \tilde{\psi}_{n}^{\dagger}(\vec{p})
$$

Substituindo as representações de Fourier em 2.143 obtemos no espaço dos momentos

$$
Z=\int \mathcal{D}(\bar{\psi}, \psi) \exp \left[\beta \sum_{n, \vec{p}} \tilde{\bar{\psi}}_{n}(\vec{p})\left(\gamma^{\mu} p_{\mu}-m+\gamma^{0} \mu\right) \tilde{\psi}_{n}(\vec{p})\right]
$$

A integral funcional pode ser então resolvida usando (B.26), fornecendo

$$
Z=\prod_{n, \vec{p}}\left[-\beta\left(\not p-m+\gamma^{0} \mu\right)\right]=\operatorname{det}\left[-\beta\left(\not p-m+\gamma^{0} \mu\right)\right]
$$

\subsubsection{O método de Faddeev e Popov}

Considere um campo bosônico descrito pela função de partição

$$
Z=\int \mathcal{D} A e^{S(A)}
$$

onde o campo $A$ representa um campo de calibre, tal como o campo de Maxwell, um campo de Yang-Mills ou o campo gravitacional. Deste modo $S(A)$ possui uma invariância por um grupo de transformação $\theta$, de tal maneira que o campo $A$ se transforma como

$$
A \rightarrow A^{\theta}
$$

mantendo a ação

$$
S\left(A^{\theta}\right)=S(A)
$$


e a medida de integração

$$
\mathcal{D} A^{\theta}=\mathcal{D} A
$$

invariantes.

A invariância da ação por 2.151) implica que configurações de campos ligadas por uma transformação de calibre são fisicamente idênticas. Assim, a integração funcional não esta bem definida pois soma sobre graus de liberdade não físicos.

Uma consequência deste fato é que não é possível extrair regras de Feynmann da integração funcional 2.150). Para ver isto, vamos notar que uma transformação infinitesimal geral possui a forma

$$
A^{\theta}=A+T_{0} \theta+T_{i}(A) \theta
$$

onde $T_{0}$ é independente de do campo $A$. Assim escrevendo a ação como

$$
S(A)=\int_{0}^{\beta} d^{d} x\left[A M A+V_{3} A A A+\cdots\right],
$$

veremos que a invariância da ação por 2.154 implica que

$$
M T_{0} \theta=0
$$

isto é, o operador da parte quadrática da ação possui um autovetor com autovalor nulo e portanto não pode ser invertido. Como o propagador está definido em termos da inversa deste operador, teremos que o propagador não pode ser definido. Logo, não é possível extrair as regras de Feynmann da integração funcional na forma (2.150).

O método mais usual para contornar este problema foi proposto por Faddeev e Popov [FP67]. A ideia consiste em fatorar a integração sobre os graus de liberdades espúrios de uma maneira invariante de calibre. Para tanto, introduzimos um escolha de calibre do tipo

$$
f A=0,
$$

com f sendo algum operador atuando em A. Em seguida introduzimos uma resolução de unidade do tipo

$$
1=\Delta(A) \int \mathcal{D} \theta \delta\left(f A^{\theta}-\sigma\right)
$$


de modo que esta expressão fixa $\Delta(A)$ como sendo o jacobiano

$$
\Delta(A)=\operatorname{det}\left[\frac{\delta f A^{\theta}}{\delta \theta}\right],
$$

que segundo 2.154 é independente de $\theta$ e possui a forma

$$
\Delta(A)=\operatorname{det}\left[f T_{0}+f T_{i}(A)\right]
$$

que é chamado de determinante de Faddeev-Popov. Este determinante possui a propriedade de ser invariante de calibre. Este fato segue diretamente da definição

$$
[\Delta(A)]^{-1}=\int \mathcal{D} \theta \delta\left(f A^{\theta}-\sigma\right)
$$

fazendo uma transformação de calibre termos

$$
\left[\Delta\left(A^{\theta^{\prime}}\right)\right]^{-1}=\int \mathcal{D} \theta \delta\left(f A^{\theta^{\prime} \theta}-\sigma\right) .
$$

Usando que as transformação de calibre possuem uma estrutura de grupo, temos que $\bar{\theta}=\theta^{\prime} \theta$ é também uma transformação de calibre. Assim, fazendo uma mudança de variáveis na integração em $\theta$ teremos

$$
\left[\Delta\left(A^{\theta^{\prime}}\right)\right]^{-1}=\int \mathcal{D} \bar{\theta} \delta\left(f A^{\bar{\theta}}-\sigma\right)=[\Delta(A)]^{-1},
$$

que nos diz que o determinante de Faddeev Popov é independente de calibre.

Deste modo, multiplicando 2.150 pela resolução da unidade 2.158 teremos

$$
Z=\int \mathcal{D} \theta \int \mathcal{D} A \Delta(A) \delta\left(f A^{\theta}-\sigma\right) e^{-S(A)} .
$$

Usando novamente que as transformações de calibre possuem uma estrutura de grupo e fazendo a transformação inversa $\theta^{-1}$ em 2.164 teremos

$$
Z=\int \mathcal{D} \theta \int \mathcal{D} A \Delta(A) \delta(f A-\sigma) e^{-S(A)},
$$

onde usamos a invariância de calibre da medida de integração da ação e de $\Delta(A)$. Por fim, iremos usar mais uma resolução da unidade do tipo

$$
1=\int \mathcal{D} \sigma \operatorname{det} N^{-1 / 2} \exp \left[-\int_{0}^{\beta} d x \sigma^{T} N \sigma\right],
$$

onde o fator $\operatorname{det} N^{-1 / 2}$ da origem ao fantasma de Nielsen-Kallosh [Kal78, Nie78]. Multi- 
plicando 2.165 por 2.166) e integrando em $\sigma$, obtemos

$$
Z=\int \mathcal{D} \theta \int \mathcal{D} A \Delta(A) \operatorname{det} N^{-1 / 2} \exp \left[S(A)-\int_{0}^{\beta} d x(f A)^{T} N(f A)\right] .
$$

Portanto, com esta construção a integração sobre o grupo de calibre se fatora, dando um fator do volume do grupo de calibre para cada ponto do espaço tempo.

Existem formalismos de fixação de calibre mais gerais que utilizam duas equações de fixação de calibre (2.157), linearmente independentes na forma

$$
\begin{aligned}
& f A=0 \\
& g A=0,
\end{aligned}
$$

resultando em um termo de fixação de calibre não quadrático na forma $(g A)^{T} N(f A)$ [BFM07]. Este formalismo de fixação de calibre geral, apesar de conter muitas complicações tal como aumentar o número de campos fantasmas e vértices de interação, é útil para obter um propagador transverso e de traço nulo para o campo do gráviton, viabilizando a soma das divergências infravermelhas para a pressão [BFMS09].

\subsubsection{Função de partição para o campo de Maxwell}

O campo de Maxwell é descrito pela densidade lagrangiana

$$
\mathcal{L}=-\frac{1}{4} F^{\mu \nu} F_{\mu \nu}
$$

onde

$$
F_{\mu \nu}=\partial_{\mu} A_{\nu}-\partial_{\nu} A_{\mu}
$$

Deste modo, a lagrangiana de Maxwell é invariante por transformações de calibre

$$
A_{\mu} \rightarrow A_{\mu}^{\theta}=A_{\mu}+\partial_{\mu} \theta,
$$

que implica que

$$
\frac{\delta A_{\mu}^{\theta}(x)}{\delta \theta(y)}=\partial_{\mu} \delta(x-y)
$$

Vamos fixar o calibre fazendo uma escolha de calibre tipo Lorentz

$$
\partial^{\mu} A_{\mu}=0 .
$$


Com esta escolha, teremos

$$
Z=\int \mathcal{D} \theta \int \mathcal{D} A \operatorname{det}\left[\partial^{\mu} \partial_{\mu} \delta(x-y)\right] \operatorname{det} N^{-1 / 2} \exp \left\{\int_{0}^{\beta} d x\left[-\frac{1}{4} F^{\mu \nu} F_{\mu \nu}-N\left(\partial^{\mu} A_{\mu}\right)^{2}\right]\right\}
$$

Neste caso, $N$ não será uma matriz, e podemos escolher $N$ como sendo uma constante. Usualmente, $N$ é expresso em termos do parâmetro de calibre $\xi$, como

$$
N=\frac{1}{2 \xi}
$$

Usando variáveis de Grassmann, podemos escrever o determinante de Faddeev-Popov [veja a equação (B.26)] como

$$
\begin{aligned}
\operatorname{det}\left[\partial_{\mu} \partial^{\mu} \delta(x-y)\right] & =\int D(\bar{C}, C) \exp \left\{\int_{0}^{\beta} d^{d} x d^{d} y\left[-\bar{C}(x) \partial_{\mu} \partial^{\mu} \delta(x-y) C(y)\right]\right\} \\
& =\int \mathcal{D}(\bar{C}, C) \exp \left\{\int_{0}^{\beta} d^{d} x \partial_{\mu} \bar{C}(x) \partial^{\mu} C(x)\right\} .
\end{aligned}
$$

Logo, na QED em um calibre tipo Lorentz temos que os campos fantasmas não interagem com o campo de calibre. A temperatura zero, temos que este termo contribuirá como uma constante multiplicativa no funcional gerador das funções de Green e pode ser desprezado. Entretanto, a temperatura finita, o determinante (2.176) é dependente da temperatura e será de fundamental importância na correta contagem de graus de liberdade do campo de calibre [Ber74], conforme veremos em detalhe na seção 4.2 .

Com isso, a função de partição terá a forma

$$
Z=\int \mathcal{D}(\bar{C}, C) \mathcal{D} A \exp \left\{\int_{0}^{\beta} d x\left[-\frac{1}{4} F^{\mu \nu} F_{\mu \nu}-\frac{1}{2 \xi}\left(\partial^{\mu} A_{\mu}\right)^{2}+\partial_{\mu} \bar{C}(x) \partial^{\mu} C(x)\right]\right\}
$$

onde estamos ignorando constantes multiplicativas em $Z$ que não dependem da temperatura. 


\section{Campo escalar a temperatura finita}

Neste capítulo estudaremos efeitos térmicos na teoria escalar. Calcularemos a função de partição do campo escalar real livre. Em seguida, consideraremos uma auto interação tipo $\phi^{4}$ e definiremos a teoria de perturbação e obteremos a primeira correção para o logaritmo da função de partição. Mostraremos que as divergências infravermelhas quebram a série perturbativa usual, sendo necessário como um primeiro passo somar sobre as divergências infravermelhas para dar sentido a série perturbativa. Verificaremos que a soma sobre as divergências infravermelhas introduzem uma não-analiticidade na pressão. O principal resultado apresentado neste capítulo está na seção 3.8, onde veremos que quanto a dimensão do espaço-tempo $d$ for par teremos uma não-analiticidade na forma $\lambda^{(d-1) / 2}$, onde $\lambda$ é a constante de acoplamento, enquanto quando $d$ for impar a nãoanaliticidade é apenas logarítmica. Nos capítulos seguintes usaremos alguns resultados que serão deduzidos neste capítulo.

\subsection{A função de partição para o campo escalar real livre}

A lagrangiana do campo escalar livre possui a forma

$$
\mathcal{L}=\frac{1}{2} \partial_{\mu} \phi \partial^{\mu} \phi-\frac{1}{2} m^{2} \phi^{2}-V(\phi)
$$

e neste caso, como estamos tratando o campo escalar neutro, não existem correntes conservadas. Como vimos na seção 2.2.2, a função de partição terá então a forma

$$
Z=\int_{\text {per. }} D \phi \exp \left(S_{E}\right),
$$


onde a integração funcional é feita sobre modos periódicos de campo, e $S_{E}$ é a ação escrita em termos do tempo imaginário

$$
\begin{aligned}
S_{E} & =\int_{0}^{\beta} d \tau \int d^{d-1} x \phi(\vec{x}, \tau)\left[\frac{1}{2}\left(\frac{\partial^{2}}{\partial \tau^{2}}+\nabla^{2}-m^{2}\right)-V(\phi)\right] \phi(\vec{x}, \tau) \\
& =\int_{0}^{\beta} d \tau \int d^{d-1} x \phi(\vec{x}, \tau)\left[\frac{1}{2}\left(-\partial^{\mu} \partial_{\mu}-m^{2}\right)-V(\phi)\right] \phi(\vec{x}, \tau),
\end{aligned}
$$

$\operatorname{com} \partial_{0}=i \partial_{\tau} \mathrm{e}$

$$
D \phi=\lim _{n, l \rightarrow \infty}\left(\frac{1}{2 \pi \Delta \tau \Delta v}\right)^{\frac{n l}{2}} d \phi_{1} \ldots d \phi_{n}
$$

é a medida da integração funcional, com os vínculos $\beta=n \Delta \tau$ e $V=l \Delta v$.

Vamos primeiro nos concentrar no caso livre, em que $V(\phi)=0$. Para tratar a integral funcional (3.2) vamos lembrar que esta se dá sobre os modos periódicos do campo $\phi$. Assim, vemos que é natural supormos que $\phi$ admite uma expansão de Fourier do tipo

$$
\begin{aligned}
\phi(\vec{x}, \tau) & =\sqrt{\frac{\beta}{V}} \sum_{n=-\infty}^{\infty} \sum_{\vec{p}} e^{i\left(\vec{p} \cdot \vec{x}+\omega_{n} \tau\right)} \tilde{\phi}_{n}(\vec{p}) \\
& =\sqrt{\frac{\beta}{V}} \sum_{n, \vec{p}} e^{i\left(x^{\mu} p_{\mu}\right)} \tilde{\phi}_{n}(\vec{p})
\end{aligned}
$$

onde $(\beta / V)^{1 / 2}$ é um fator que fixa a dimensão de $\phi$ (de forma que $\tilde{\phi}_{n}(\vec{p})$ seja adimensional), no formalismo do tempo imaginário temos $x^{0}=-i \tau, p_{0}=i \omega_{n}=\frac{2 n \pi i}{\beta}=2 n \pi T$ são as freqüências de Matsubara para bósons e $T=1 / \beta$ é a temperatura. Note que é necessário requerer que $\tilde{\phi}_{-n}^{*}(-\vec{p})=\tilde{\phi}_{n}(\vec{p})$ para que o campo $\phi$ seja real. Neste ponto, estamos considerando o campo escalar em um caixa hipercúbica de lado $L$ e volume $V=L^{d-1}$, com condições periódicas de contorno, de modo que

$$
\vec{p}=\frac{2 \pi}{L} \vec{l}
$$

$\operatorname{com} l_{i} \in \mathbb{Z}$

Na representação de Fourier a ação livre é reescrita como

$$
\begin{aligned}
S_{0} & =\int_{0}^{\beta} d \tau \int d^{d-1} x\left[\frac{1}{2} \partial^{\mu} \phi \partial_{\mu} \phi-\frac{m^{2}}{2} \phi^{2}\right] \\
& =-\frac{\beta}{2 V} \int_{0}^{\beta} d \tau \int d^{d-1} x \sum_{n, p} \sum_{n^{\prime}, p^{\prime}} e^{i\left[\left(\vec{p}+\overrightarrow{p^{\prime}}\right) \cdot \vec{x}+\left(\omega_{n}+\omega_{n^{\prime}}\right) \tau\right]}\left(p^{\mu} p_{\mu}^{\prime}+m^{2}\right) \tilde{\phi}_{n}(\vec{p}) \tilde{\phi}_{n^{\prime}}\left(\overrightarrow{p^{\prime}}\right) \\
& =-\frac{\beta^{2}}{2} \sum_{n, \vec{p}}\left(-p^{2}+m^{2}\right) \tilde{\phi}_{n}(\vec{p}) \tilde{\phi}_{-n}(-\vec{p}),
\end{aligned}
$$


onde usamos que

$$
\int_{0}^{\beta} d \tau \int d^{d-1} x e^{\left.i\left(\vec{p}+\overrightarrow{p^{\prime}}\right) \cdot \vec{x}+\left(\omega_{n}+\omega_{n^{\prime}}\right) \tau\right)}=\beta V \delta_{0}^{n+n^{\prime}} \delta_{0}^{\vec{p}+\vec{p}^{\prime}}
$$

Variar a forma funcional de uma função periódica é equivalente a variar os coeficientes de Fourier da sua expansão (veja o apêndice A). Assim usaremos

$$
\int \mathcal{D} \phi=\prod_{n, \vec{p}} \int_{-\infty}^{\infty} d \phi_{n}(\vec{p})
$$

Esta expressão será tomada como a definição da medida de integração funcional durante o restante do trabalho, pois como (3.7) mostra a base de Fourier diagonaliza a parte quadrática da ação.

Substituindo (3.9) e (3.7) em (3.2), vemos que função de partição livre pode ser escrita como

$$
Z_{0}=\prod_{n=-\infty}^{\infty} \prod_{\vec{p}} \int_{-\infty}^{\infty} d \tilde{\phi}_{n}(\vec{p}) \exp \left[-\frac{\beta^{2}}{2}\left(\omega_{n}^{2}+\omega^{2}\right)\left|\tilde{\phi}_{n}(\vec{p})\right|^{2}\right]
$$

com $\omega^{2}=\vec{p}^{2}+m^{2}$. A 3.10 é um produtório de integrais gaussianas que fornece

$$
Z_{0}=\prod_{n=-\infty}^{\infty} \prod_{\vec{p}}\left[\frac{2 \pi}{\beta^{2}\left(\omega_{n}^{2}+\omega^{2}\right)}\right]^{\frac{1}{2}}
$$

Portanto, extraindo o logaritmo na expressão 3.11 escrevemos o produtório como uma soma na forma

$$
\log Z_{0}=-\frac{1}{2} \sum_{n} \sum_{\vec{p}} \log \left[\beta^{2} \omega^{2}+(2 \pi n)^{2}\right]
$$

Note que todas as constantes aditivas independentes da temperatura podem ser desprezadas. No limite termodinâmico $V \rightarrow \infty$ a expressão 3.6 mostra que a soma em $\vec{p}$ recairá em uma integral como

$$
\sum_{\vec{p}} \frac{1}{V} \rightarrow \int \frac{d^{d-1} p}{(2 \pi)^{d-1}}
$$

e assim reescrevemos 3.12 como

$$
\log Z_{0}=-\frac{V}{2} \sum_{n} \int \frac{d^{d-1} p}{(2 \pi)^{d-1}} \log \left[\beta^{2} \omega^{2}+(2 \pi n)^{2}\right] .
$$


Com o fim de calcular a soma em (3.14), vamos utilizar a identidade

$$
\int_{1}^{\beta^{2} \omega^{2}} \frac{d x^{2}}{x^{2}+(2 n \pi)^{2}}=\log \left[\beta^{2} \omega^{2}+(2 \pi n)^{2}\right]-\log \left[1+(2 \pi n)^{2}\right] .
$$

No lado direito da igualdade acima, o primeiro termo é o integrando de (3.14) e o segundo é independente da temperatura. Desta forma, substituindo (3.15) em (3.14), reescrevemos esta como

$$
\log Z_{0}=-\frac{V}{2} \sum_{n} \int \frac{d^{d-1} p}{(2 \pi)^{d-1}} \int_{1}^{\beta^{2} \omega^{2}} \frac{d x^{2}}{x^{2}+(2 n \pi)^{2}} .
$$

Comutando a soma com as integrais obtemos

$$
\log Z_{0}=-\frac{V}{2} \int \frac{d^{d-1} p}{(2 \pi)^{d-1}} \int_{1}^{\beta^{2} \omega^{2}} d x \sum_{n} \frac{1}{x+(2 n \pi)^{2}}
$$

Podemos calcular o limite da série transformando-a em uma integral, tal como será apresentado no apêndice C [veja a equação (C.21)]. Desta maneira, a soma é escrita como

$$
\sum_{n=-\infty}^{\infty} \frac{1}{c^{2}+(2 n \pi)^{2}}=\frac{1}{4 \pi}\left(\int_{-\infty-i \epsilon}^{\infty-i \epsilon} d z+\int_{\infty+i \epsilon}^{-\infty+i \epsilon} d z\right) \frac{\cot (z / 2)}{c^{2}+z^{2}}
$$

Fechando o contorno da primeira integral no semiplano inferior e o da segunda integral no semiplano inferior (com $1 /\left(z^{2}+c^{2}\right)$ garantindo a convergência) e usando o teorema de resíduos, obtemos:

$$
\begin{aligned}
\int_{-\infty-i \epsilon}^{\infty-i \epsilon} d z \frac{\cot (z / 2)}{c^{2}+z^{2}} & =\frac{2 \pi i}{2 i c} \cot \left(\frac{i c}{2}\right) \\
\int_{\infty+i \epsilon}^{-\infty+i \epsilon} d z \frac{\cot (z / 2)}{c^{2}+z^{2}} & =\frac{2 \pi i}{-2 i c} \cot \left(-\frac{i c}{2}\right)
\end{aligned}
$$

Substituindo esses resultados em (3.18), obtemos

$$
\sum_{n=-\infty}^{\infty} \frac{1}{c^{2}+(2 n \pi)^{2}}=\frac{1}{2 c} \operatorname{coth} \frac{c}{2}
$$

Reescrevendo a coth e substituindo em 3.17) escrevemos $\log Z$ como

$$
\log Z_{0}=-\frac{V}{2} \int \frac{d^{d-1} p}{(2 \pi)^{d-1}} \int_{1}^{\beta^{2} \omega^{2}} d x^{2} \frac{1}{2 x}\left(1+\frac{2}{e^{x}-1}\right) .
$$

Negligenciando as constantes independentes da temperatura que não terão relevância física, obtemos

$$
\log Z_{0}=V \int \frac{d^{d-1} p}{(2 \pi)^{d-1}}\left[-\frac{\beta \omega}{2}-\log \left(1-e^{-\beta \omega}\right)\right] .
$$

Esta é a função de partição para o campo escalar livre. O primeiro termo vem da energia 
de ponto zero do oscilador harmônico. A energia associada a este termo tem a forma

$$
E_{\text {vácuo }}=-\frac{\partial}{\partial \beta} \log Z_{\text {vácuo }}=V \int \frac{d^{d-1} p}{(2 \pi)^{d-1}} \frac{\omega}{2},
$$

ou seja, esta energia do vácuo que está inclusa em (3.22 possui um valor infinito. Assim, definimos as energias em relação a energia do vácuo de modo a remover a divergência. A pressão do vácuo, que possui a forma

$$
p_{\text {vácuo }}=\frac{1}{\beta} \frac{\partial}{\partial V} \log Z_{\text {vácuo }}=-\frac{E_{\text {vácuo }}}{V},
$$

também apresenta divergência. Desta forma a pressão também será redefinida em relação a pressão do vácuo.

Ignorando o termo de vácuo, teremos

$$
\begin{aligned}
\log Z_{0} & =-V \int \frac{d^{d-1} p}{(2 \pi)^{d-1}} \log \left(1-e^{-\beta \omega}\right) \\
& =-\frac{V \Omega_{d-1}}{(2 \pi)^{d-1}} \int_{0}^{\infty} d|\vec{p}||\vec{p}|^{d-2} \log \left(1-e^{-\beta \omega}\right) .
\end{aligned}
$$

No limite de massa nula, que será o termo dominante no limite de altas temperaturas, teremos

$$
\begin{aligned}
\log Z_{0} & =-\frac{V \Omega_{d-1}}{(2 \pi)^{d-1}} \int_{0}^{\infty} d|\vec{p}||\vec{p}|^{d-2} \log \left(1-e^{-\beta|\vec{p}|}\right) \\
& =-\frac{V \Omega_{d-1} T^{d-1}}{(2 \pi)^{d-1}} \int_{0}^{\infty} d x x^{d-2} \log \left(1-e^{-x}\right) .
\end{aligned}
$$

Fazendo uma integração por partes reescrevemos

$$
\begin{aligned}
\log Z_{0} & =\frac{V \Omega_{d-1} T^{d-1}}{(2 \pi)^{d-1}(d-1)} \int_{0}^{\infty} d x x^{d-1} \frac{e^{-x}}{1-e^{-x}} \\
& =\frac{V \Omega_{d-1} T^{d-1}}{(2 \pi)^{d-1}(d-1)} \sum_{n=1}^{\infty} \int_{0}^{\infty} d x x^{d-1} e^{-n x} \\
& =\frac{V \Omega_{d-1} T^{d-1}}{(2 \pi)^{d-1}(d-1)} \sum_{n=1}^{\infty} \frac{1}{n^{d}} \int_{0}^{\infty} d x x^{d-1} e^{-x}
\end{aligned}
$$

onde podemos reconhecer as funções gama de Euler e zeta de Riemann, obtendo

$$
\log Z_{0}=\frac{V \Omega_{d-1} T^{d-1} \zeta(d) \Gamma(d)}{(2 \pi)^{d-1}(d-1)}
$$


e a respectiva forma para a pressão será

$$
\begin{aligned}
P & =\frac{\Omega_{d-1} T^{d} \zeta(d) \Gamma(d)}{(2 \pi)^{d-1}(d-1)} \\
& =\frac{T^{d} \zeta(d) \Gamma(d-1)}{2^{d-2} \pi^{(d-1) / 2} \Gamma\left(\frac{d-1}{2}\right)}
\end{aligned}
$$

onde substituímos o ângulo sólido em $D$ dimensões

$$
\Omega_{D}=\frac{2 \pi^{D / 2}}{\Gamma(D / 2)}
$$

Em $d=4$, a pressão tem a forma [KG06]

$$
P=\frac{T^{4} \pi^{2}}{90}
$$

\subsection{Interações e teoria de perturbação}

Até agora consideramos o caso de campos livres de interação. No entanto, agora consideraremos uma ação $S$ do tipo

$$
S[\phi]=S_{0}[\phi]+S_{i}[\phi],
$$

onde $S_{0}$ é a parte quadrática da ação e $S_{i}$ é a parte de interação que contem potências do campo $\phi$ maiores que dois. A função de partição, neste caso, pode ser escrita como

$$
Z=\int D \phi \exp \left\{S_{0}[\phi]+S_{i}[\phi]\right\}=\int D \phi \exp \left\{S_{0}[\phi]\right\} \exp \left\{S_{i}[\phi]\right\}
$$

com a integral sobre configurações periódicas do campo e a ação escrita no tempo imaginário.

A teoria de perturbação baseia-se no fato que o termo de interação $S_{i}$ é pequeno em um sentido que definiremos adiante. Deste modo, expandindo $\exp \left\{S_{i}[\phi]\right\}$ em série de Taylor e tomando o logaritmo membro a membro obtemos

$$
\log (Z)=\log \left\{\int D \phi \exp \left(S_{0}[\phi]\right) \sum_{l=0}^{\infty} \frac{S_{i}^{l}[\phi]}{l !}\right\}
$$

que pode ser reescrita de maneira mais conveniente como

$$
\log (Z)=\log \left[\int D \phi \exp \left(S_{0}\right)\right]+\log \left[1+\frac{\int D \phi \exp \left(S_{0}\right) \sum_{l=1}^{\infty} S_{i}^{l}}{l ! \int D \phi \exp \left(S_{0}\right)}\right] .
$$

Segundo a Mecânica Estatística, o valor médio de um operador $O$ em um ensemble 
descrito por $S_{0}$ é dado por

$$
\left\langle T_{\tau}[O]\right\rangle_{S_{0}}=\frac{\operatorname{Tr} \rho_{0} T_{\tau}[O]}{\operatorname{Tr} \rho_{0}}
$$

onde $\rho_{0}$ é a matriz densidade no caso livre, e $T_{\tau}[\cdot]$ é o operador de ordenação temporal no formalismo do tempo imaginário. A equação (2.115) implica que no formalismo da integração funcional o valor esperado 3.36 possui a forma

$$
\left\langle T_{\tau} O\right\rangle_{S_{0}}=\frac{\int D \phi \exp \left(S_{0}\right) O}{\int D \phi \exp \left(S_{0}\right)}
$$

que mostra que as médias são calculadas sobre todas as configurações de campo possíveis, com o peso estatístico dado da exponencial da ação.

Assim podemos escrever 3.35 como

$$
\log (Z)=\log \left(Z_{0}\right)+\log \left(Z_{i}\right)=\log \left(Z_{0}\right)+\log \left(1+\sum_{l=1}^{\infty} \frac{\left\langle S_{i}^{l}\right\rangle_{S_{0}}}{l !}\right)
$$

onde iremos omitir o operador de ordenação temporal, estando subentendido que as médias calculadas no formalismo da integração funcional são ordenadas no tempo. O segundo logaritmo do lado esquerdo pode ser expandido em serie de Taylor obtendo

$$
\log \left(Z_{i}\right)=\sum_{l=1}^{\infty} \frac{\left\langle S_{i}^{l}\right\rangle_{S_{0}}}{l !}-\frac{1}{2}\left(\sum_{l=1}^{\infty} \frac{\left\langle S_{i}^{l}\right\rangle_{S_{0}}}{l !}\right)^{2}+\ldots
$$

que é a expressão da teoria de perturbação para a função de partição. Agora dizemos que $S_{i}$ é pequeno se $1 \gg\left\langle S_{i}^{n}\right\rangle_{S_{0}} \gg\left\langle S_{i}^{n+1}\right\rangle_{S_{0}}$, para que a expansão perturbativa faça sentido.

Vamos, a partir de agora, considerar o campo escalar neutro com uma interação do tipo

$$
\mathcal{L}_{\text {int }}=-\frac{\lambda}{4 !} \phi^{4}
$$

A contribuição de primeira ordem em $\lambda$ para $\log Z$ pode ser obtida de (3.39) como

$$
\log \left(Z_{1}\right)=\left\langle S_{i}\right\rangle_{S_{0}}=-\frac{\lambda}{4 !} \frac{\int D \phi e^{S_{0}} \int_{0}^{\tau} d \tau \int d^{d-1} x \phi^{4}(\vec{x}, \tau)}{\int D \phi e^{S_{0}}} .
$$

Escrevendo o campo $\phi$ na base de Fourier (3.5) e substituindo (3.7) e (3.9) na expressão 
(3.41) obtemos

$$
\begin{aligned}
& \log \left(Z_{1}\right)=-\lambda Z_{0}^{-1}\left(\frac{\beta}{V}\right)^{2} \prod_{l, \vec{q}} \int d \tilde{\phi}_{l}(\vec{q}) e^{-\frac{\beta^{2}}{2}\left(\omega_{l}^{2}+\vec{q}^{2}+m^{2}\right) \tilde{\phi}_{l}(\vec{q}) \tilde{\phi}_{-l}(-\vec{q})} \\
& \times \sum_{n_{1}, \overrightarrow{p_{1}}, \ldots, n_{4}, \overrightarrow{p_{4}}} \int_{0}^{\beta} d \tau \int d^{d-1} x e^{i\left(\overrightarrow{p_{1}}+\ldots+\overrightarrow{p_{4}}\right) \cdot \vec{x}+i\left(\omega_{n_{1}}+\ldots+\omega_{n_{4}}\right) \tau} \frac{\tilde{\phi}_{n_{1}}\left(\overrightarrow{p_{1}}\right) \ldots \tilde{\phi}_{n_{4}}\left(\overrightarrow{p_{4}}\right)}{4 !}
\end{aligned}
$$

onde

$$
Z_{0}=\prod_{l, \vec{q}} \int d \tilde{\phi}_{l}(\vec{q}) e^{-\frac{\beta^{2}}{2}\left(\omega_{l}^{2}+\vec{q}^{2}+m^{2}\right) \tilde{\phi}_{l}(\vec{q}) \tilde{\phi}_{-l}(-\vec{q})}
$$

é a função de partição livre. As integrais em $d^{d-1} x$ e $d \tau$ podem então ser resolvidas em termos de função $\delta$ de conservação de momento-frequência. Fazendo isto, $\log \left(Z_{1}\right)$ será dado por

$$
\begin{aligned}
\log \left(Z_{1}\right) & =-\lambda Z_{0}^{-1} \frac{\beta^{3}}{V} \prod_{l, \vec{q}} \int d \tilde{\phi}_{l}(\vec{q}) e^{-\frac{\beta^{2}}{2}\left(\omega_{l}^{2}+\vec{q}^{2}+m^{2}\right) \tilde{\phi}_{l}(\vec{q}) \tilde{\phi}_{-l}(-\vec{q})} \\
& \times \sum_{n_{1}, \overrightarrow{p_{1}}, \ldots, n_{4}, \overrightarrow{p_{4}}} \delta_{0}^{\overrightarrow{p_{1}}+\ldots+\overrightarrow{p_{4}}} \delta_{0}^{n_{1}+\ldots+n 4} \frac{\tilde{\phi}_{n_{1}}\left(\overrightarrow{p_{1}}\right) \ldots \tilde{\phi}_{n_{4}}\left(\overrightarrow{p_{4}}\right)}{4 !} .
\end{aligned}
$$

O produtório no par de índices $l \vec{q}$ corre sobre todos os valores possíveis dos índices. Portanto, devido ao caráter par do argumento da exponencial que aparece no integrando, as somas em $\left(n_{1}, \vec{p}_{1}\right), \ldots\left(n_{4}, \vec{p}_{4}\right)$ só darão contribuição não nula na integral quando $n_{1}=$ $-n_{2}, \vec{p}_{1}=-\vec{p}_{2}, n_{3}=-n_{4}, \vec{p}_{3}=-\vec{p}_{4}$ e as outras duas permutações equivalentes. Como existem três permutações possíveis nos índices, vemos que teremos um fator combinatorial três, resultando em

$$
\begin{aligned}
\log \left(Z_{1}\right) & =-3 \lambda N \frac{\beta^{3}}{V} \prod_{l, \vec{q}} \int d \tilde{\phi}_{l}(\vec{q}) e^{-\frac{\beta^{2}}{2}\left(\omega_{l}^{2}+\vec{q}^{2}+m^{2}\right) \tilde{\phi}_{l}(\vec{q}) \tilde{\phi}_{-l}(-\vec{q})} \\
& \times \sum_{n_{1}, \overrightarrow{p_{1}, n_{2}, \overrightarrow{p_{2}}}} \frac{\tilde{\phi}_{n_{1}}\left(\vec{p}_{1}\right) \tilde{\phi}_{-n_{1}}\left(-\vec{p}_{1}\right) \tilde{\phi}_{n_{2}}\left(\vec{p}_{2}\right) \tilde{\phi}_{-n_{2}}\left(-\vec{p}_{2}\right)}{4 !},
\end{aligned}
$$

Para cada termo particular da soma em $n_{1}, \vec{p}_{1}, n_{2}$ e $\vec{p}_{2}$ teremos que as integrais em $d \tilde{\phi}_{n_{1}}\left(\vec{p}_{1}\right)$ e $d \tilde{\phi}_{n_{2}}\left(\vec{p}_{2}\right)$ irão se fatorar. As outras integrais em $d \tilde{\phi}_{l}(\vec{q})$, para $n_{1}, n_{2} \neq l$ ou $\vec{p}_{1}, \vec{p}_{2} \neq l$ irão se cancelar com o denominador $Z_{0}$. Desta forma teremos:

$$
\log \left(Z_{1}\right)=-3 \frac{\lambda}{4 !} \frac{\beta^{3}}{V}\left[\sum_{n, \vec{p}} \frac{\int d \tilde{\phi}_{n}(\vec{p}) e^{-\frac{\beta^{2}}{2}\left(\omega_{n}^{2}+\vec{p}^{2}+m^{2}\right) \tilde{\phi}_{n}(\vec{p}) \tilde{\phi}_{-n}(-\vec{p})} \tilde{\phi}_{n}(\vec{p}) \tilde{\phi}_{-n}(-\vec{p})}{\int d \tilde{\phi}_{n}(\vec{p}) e^{-\frac{\beta^{2}}{2}\left(\omega_{n}^{2}+\vec{p}^{2}+m^{2}\right) \tilde{\phi}_{n}(\vec{p}) \tilde{\phi}_{-n}(-\vec{p})}}\right]^{2}
$$

Lembrando que a realidade do campo $\phi$ implica em $\tilde{\phi}_{n}(\vec{p})=\tilde{\phi}_{-n}^{*}(-\vec{p})$, vemos que as 
integrais acima são do tipo:

$$
\frac{\int d x e^{-M|x|^{2}}|x|^{2}}{\int d x e^{-M|x|^{2}}}=-\frac{d}{d M} \log \left[\int d x e^{-M|x|^{2}}\right]=\frac{1}{2 M} .
$$

Desta maneira, podemos resolver as integrais (3.46), obtendo

$$
\log \left(Z_{1}\right)=-3 \frac{\lambda}{4 !} \frac{\beta^{3}}{V}\left[\frac{1}{\beta^{2}} \sum_{n, \vec{p}} D_{0}\left(\omega_{n}, \vec{p}\right)\right]^{2}
$$

No limite termodinâmico 3.13$) \log Z_{1}$ tem a forma

$$
\log \left(Z_{1}\right)=-3 \frac{\lambda}{4 !} \beta V\left[\frac{1}{\beta} \sum_{n} \int \frac{d^{d-1} p}{(2 \pi)^{d-1}} D_{0}\left(\omega_{n}, \vec{p}\right)\right]^{2},
$$

onde

$$
D_{0}\left(\omega_{n}, \vec{p}\right)=\frac{1}{\omega_{n}^{2}+\vec{p}^{2}+m^{2}}=\frac{-1}{p^{2}-m^{2}},
$$

é o propagador livre do campo escalar.

Vamos agora calcular a contribuição de segunda ordem em $\lambda$ para $\log \left(Z_{i}\right)$. Segundo 3.39 essa contribuição é dada por

$$
\log \left(Z_{2}\right)=1 / 2\left\langle S_{i}^{2}\right\rangle_{S_{0}}-1 / 2\left\langle S_{i}\right\rangle_{S_{0}}^{2}
$$

O termo $\left\langle S_{i}\right\rangle_{S_{0}}^{2}$ pode ser obtido diretamente de 3.49 de forma que resta calcular o termo $\left\langle S_{i}^{2}\right\rangle_{S_{0}}$. Vamos usar 3.36 para escrever $\left\langle S_{i}^{2}\right\rangle_{S_{0}}$ como

$$
\left\langle S_{i}^{2}\right\rangle_{S_{0}}=\frac{\int d \phi e^{S_{0}} S_{i}^{2}}{\int d \phi e^{S_{0}}}
$$

Aplicando expansão de Fourier para o campo 3.5 escrevemos este valor esperado como

$$
\begin{aligned}
& \left\langle S_{i}^{2}\right\rangle_{S_{0}}=\frac{\lambda^{2} \beta^{6} Z_{0}^{-1}}{V^{2}} \prod_{l, \vec{q}} \int d \tilde{\phi}_{l}(\vec{q}) e^{-\frac{\beta^{2}}{2}\left(\omega_{l}^{2}+\vec{q}^{2}+m^{2}\right) \tilde{\phi}_{l}(\vec{q}) \tilde{\phi}_{-l}(-\vec{q})} \sum_{n_{1}, \overrightarrow{p_{1}, \ldots, n_{4}, \overrightarrow{p_{4}}}} \sum_{m_{1}, \overrightarrow{k_{1}}, \ldots, m_{4}, \overrightarrow{k_{4}}} \\
& \times\left[\delta_{0}^{\overrightarrow{p_{1}}+\ldots+\overrightarrow{p_{4}}} \delta_{0}^{n_{1}+\ldots+n_{4}} \delta_{0}^{\overrightarrow{k_{1}}+\ldots+\overrightarrow{k_{4}}} \delta_{0}^{m_{1}+\ldots+m_{4}} \frac{\tilde{\phi}_{n_{1}}\left(\overrightarrow{p_{1}}\right) \ldots \tilde{\phi}_{n_{4}}\left(\overrightarrow{p_{4}}\right)}{4 !} \frac{\tilde{\phi}_{m_{1}}\left(\overrightarrow{k_{1}}\right) \ldots \tilde{\phi}_{m_{4}}\left(\overrightarrow{k_{4}}\right)}{4 !}\right] .
\end{aligned}
$$

Novamente, por argumentos de paridade, vemos que os únicos termos das somas que fornecem uma contribuição não nula nas integrações funcionais são os termos cujos conjuntos 
de índices obedecem

$$
\begin{aligned}
& n_{1}=-n_{2}, p_{1}=-p_{2}, n_{3}=-n_{4}, p_{3}=-p_{4} \\
& m_{1}=-m_{2}, k_{1}=-k_{2}, m_{3}=-m_{4}, k_{3}=-k_{4}, \\
& n_{1}=-m_{1}, p_{1}=-k_{1}, n_{2}=-m_{2}, p_{2}=-k_{2} \\
& n_{3}=-n_{4}, p_{3}=-p_{4}, m_{3}=-m_{4}, k_{3}=-k_{4},
\end{aligned}
$$

$\mathrm{Ou}$

$$
\begin{aligned}
& n_{1}=-m_{1}, p_{1}=-k_{1}, n_{2}=-m_{2}, p_{2}=-k_{2} \\
& n_{3}=-m_{3}, p_{3}=-k_{3}, n_{4}=-m_{4}, p_{4}=-k_{4},
\end{aligned}
$$

e as outras permutações dentro de cada um dos 3 casos resultarão em uma contribuição equivalente. Assim o número de permutações equivalentes dentro de um mesmo caso irá aparecer como um fator combinatorial que conta as maneiras distintas de se obter a mesma contribuição.

Contando os fatores combinatoriais reescrevemos 3.53 como

$$
\begin{aligned}
\left\langle S_{i}^{2}\right\rangle_{S_{0}} & =\frac{\lambda^{2} \beta^{6} Z_{0}^{-1}}{(4 !)^{2} V^{2}} \prod_{l, \vec{q}} \int d \tilde{\phi}_{l}(\vec{q}) e^{-\frac{\beta^{2}}{2}\left(\omega_{l}^{2}+\vec{q}^{2}+m^{2}\right) \tilde{\phi}_{l}(\vec{q}) \tilde{\phi}_{-l}(-\vec{q})} \\
& \times\left\{9\left[\sum_{n_{1}, \overrightarrow{p_{1}, n_{2}, \overrightarrow{p_{2}}}} \tilde{\phi}_{n_{1}}\left(\overrightarrow{p_{1}}\right) \tilde{\phi}_{-n_{1}}\left(-\overrightarrow{p_{1}}\right) \tilde{\phi}_{n_{2}}\left(\overrightarrow{p_{2}}\right) \tilde{\phi}_{-n_{2}}\left(-\overrightarrow{p_{2}}\right)\right]^{2}\right. \\
& +72\left[\sum_{n_{1}, \overrightarrow{p_{1}}} \tilde{\phi}_{n_{1}}\left(\overrightarrow{p_{1}}\right) \tilde{\phi}_{-n_{1}}\left(-\overrightarrow{p_{1}}\right)\right]^{2} \\
& \times\left[\sum_{n_{1}, \overrightarrow{p_{1}, n_{2}, \overrightarrow{p_{2}}}} \delta_{0}^{p_{1}+\overrightarrow{p_{2}}} \delta_{0}^{n_{1}+n_{2}} \tilde{\phi}_{n_{1}}\left(\overrightarrow{p_{1}}\right) \tilde{\phi}_{-n_{1}}\left(-\overrightarrow{p_{1}}\right) \tilde{\phi}_{n_{2}}\left(\overrightarrow{p_{2}}\right) \tilde{\phi}_{-n_{2}}\left(-\overrightarrow{p_{2}}\right)\right] \\
& \left.+24\left[\sum_{n_{1}, \overrightarrow{p_{1}, \ldots, n_{4}, \vec{p}_{4}}} \delta_{0}^{n_{1}+\ldots+n_{4}} \delta_{0}^{\vec{p}_{1}+\ldots+\vec{p}_{4}} \tilde{\phi}_{n_{1}}\left(\overrightarrow{p_{1}}\right) \tilde{\phi}_{-n_{1}}\left(-\overrightarrow{p_{1}}\right) \ldots \tilde{\phi}_{n_{4}}\left(\overrightarrow{p_{4}}\right) \tilde{\phi}_{-n_{4}}\left(-\overrightarrow{p_{4}}\right)\right]\right\} .
\end{aligned}
$$


e resolvendo as integrais do mesmo tipo das que aparecem em 3.47) obtemos:

$$
\begin{aligned}
\left\langle S_{i}^{2}\right\rangle_{S_{0}} & =\frac{\lambda^{2} \beta^{6}}{(4 !)^{2} V^{2}}\left[9\left(\sum_{n, \vec{p}} \frac{1}{\beta^{2}\left(\omega_{n}^{2}+\vec{p}^{2}+m^{2}\right)}\right)^{4}\right. \\
& +72\left(\sum_{n, \vec{p}} \frac{1}{\beta^{2}\left(\omega_{n}^{2}+\vec{p}^{2}+m^{2}\right)}\right)^{2} \\
& \times\left(\sum_{n_{1}, \vec{p}_{1}, n_{2}, \vec{p}_{2}} \frac{\delta_{0}^{n_{1}+n_{2}} \delta_{0}^{\vec{p}_{1}+\vec{p}_{2}}}{\beta^{4}\left(\omega_{n_{1}}^{2}+\vec{p}_{1}^{2}+m^{2}\right)\left(\omega_{n_{2}}^{2}+\vec{p}_{2}^{2}+m^{2}\right)}\right) \\
& \left.+24\left(\sum_{n_{1}, \ldots, n_{4}, \vec{p}_{1}, \ldots \vec{p}_{4}} \frac{\delta_{0}^{n_{1}+\ldots+n_{4}} \delta_{0}^{\vec{p}_{1}+\ldots+\vec{p}_{4}}}{\beta^{2}\left(\omega_{n_{1}}^{2}+\vec{p}_{1}^{2}+m^{2}\right) \ldots \beta^{2}\left(\omega_{n_{4}}^{2}+\vec{p}_{4}^{2}+m^{2}\right)}\right)\right]
\end{aligned}
$$

No limite do contínuo identificamos

$$
V \delta_{0}^{\vec{p}} \rightarrow(2 \pi)^{d-1} \delta^{(d-1)}(\vec{p})
$$

$\mathrm{e}$

$$
\int d^{d-1} x=V
$$

Deste modo, a contribuição de segunda ordem em $\lambda$ para $\log Z$ é reescrita como

$$
\begin{aligned}
\left\langle S_{i}^{2}\right\rangle_{S_{0}} & =\frac{\lambda^{2} \beta^{2} V^{2}}{(4 !)^{2}}\left[9\left(\sum_{n} \int \frac{d^{d-1} p}{\beta(2 \pi)^{d-1}} D_{0}\left(\omega_{n}, \vec{p}\right)\right)^{4}\right. \\
& +72\left(\sum_{n} \int \frac{d^{d-1} p}{\beta(2 \pi)^{d-1}} D_{0}\left(\omega_{n}, \vec{p}\right)\right)^{2} \\
& \times\left(\sum_{l_{1}, l_{2}} \int \frac{d^{d-1} q_{1}}{\beta(2 \pi)^{d-1}} \frac{d^{d-1} q_{2}}{\beta(2 \pi)^{d-1}} D_{0}\left(\omega_{l_{1}}, \vec{q}_{1}\right) D_{0}\left(\omega_{l_{2}}, \vec{q}_{2}\right) \frac{(2 \pi)^{d-1} \delta\left(\vec{q}_{1}+\vec{q}_{2}\right)}{V} \delta_{0}^{l_{1}+l_{2}}\right) \\
& +24\left(\sum_{l_{1}, \ldots l_{4}} \int \frac{d^{d-1} q_{1}}{\beta(2 \pi)^{d-1}} \ldots \frac{d^{d-1} q_{4}}{\beta(2 \pi)^{d-1}} D_{0}\left(\omega_{l_{1}}, \vec{q}_{1}\right) \ldots D_{0}\left(\omega_{l_{4}}, \vec{q}_{4}\right)\right. \\
& \left.\left.\times \frac{(2 \pi)^{d-1} \delta\left(\vec{q}_{1}+\ldots+\vec{q}_{4}\right)}{V} \delta_{0}^{l_{1}+\ldots+l_{4}}\right)\right] .
\end{aligned}
$$

A expressão para $\left\langle S_{i}\right\rangle_{S_{0}}$ já foi calculada e é dada por 3.49). Lembrando da expressão (3.51) para $\log \left(Z_{2}\right)$, vemos que o termo $-1 / 2\left\langle S_{i}\right\rangle_{S_{0}}^{2}$ cancela o primeiro termo de (3.61). 
Assim, $\log \left(Z_{2}\right)$ pode ser escrito como

$$
\begin{aligned}
\log \left(Z_{2}\right) & =\left(\frac{-\lambda}{4 !}\right)^{2} \frac{\beta V}{2}\left[72\left(\sum_{n} \int \frac{d^{d-1} p}{\beta(2 \pi)^{d-1}} D_{0}\left(\omega_{n}, \vec{p}\right)\right)^{2}\right. \\
& \times\left(\sum_{l_{1}, l_{2}} \int \frac{d^{d-1} q_{1}}{\beta(2 \pi)^{d-1}} \frac{d^{d-1} q_{2}}{\beta(2 \pi)^{d-1}} D_{0}\left(\omega_{l_{1}}, \vec{q}_{1}\right) D_{0}\left(\omega_{l_{2}}, \vec{q}_{2}\right)(2 \pi)^{d-1} \delta\left(\vec{q}_{1}+\vec{q}_{2}\right) \beta \delta_{0}^{l_{1}+l_{2}}\right) \\
& +24\left(\sum_{l_{1}, \ldots, l_{4}} \int \frac{d^{d-1} q_{1}}{\beta(2 \pi)^{d-1}} \ldots \frac{d^{d-1} q_{4}}{\beta(2 \pi)^{d-1}} D_{0}\left(\omega_{l_{1}}, \vec{q}_{1}\right) \ldots D_{0}\left(\omega_{l_{4}}, \vec{q}_{4}\right)\right. \\
& \left.\left.\times(2 \pi)^{d-1} \delta\left(\vec{q}_{1}+\ldots+\vec{q}_{4}\right) \beta \delta_{0}^{l_{1}+\ldots+l_{4}}\right)\right] .
\end{aligned}
$$

Uma contribuição do tipo $\left\langle S_{i}\right\rangle_{S_{0}}^{2}$ é chamada de desconexa, pois possui duas partes conexas independentes $\left\langle S_{i}\right\rangle_{S_{0}} \times\left\langle S_{i}\right\rangle_{S_{0}}$. Até ordem $\lambda^{2}$ verificamos que apenas termos conexos contribuem para $\log Z$. Na próxima seção mostraremos que a parte desconexa de $\left\langle S_{i}^{l}\right\rangle_{S_{0}}$ não contribui para $\log Z$ em nenhuma ordem de teoria de perturbação. Assim escrevendo $Z=e^{W}$, vemos que $W$ é uma soma de diagramas conexos.

\subsection{A pressão em termos de diagramas conexos}

Na seção anterior vimos que apenas diagramas conexos contribuem para $\log Z$, até ordem $\lambda^{2}$. Agora vamos provar que apenas diagramas conexos contribuem para o logaritmo da função de partição $\log Z$ em todas as ordens de teoria de perturbação. Partiremos da expressão 3.33 reescrita como

$$
Z=Z_{0} \sum_{n=0}^{\infty} \frac{\left\langle S_{i}^{n}\right\rangle_{S_{0}}}{n !}
$$

Podemos expressar $\left\langle S_{i}^{n}\right\rangle_{S_{0}}$ como uma soma de diagramas desconexos, que por sua vez, são escritos como o produto de partes conexas, tais como

$$
\left\langle S_{i}^{n}\right\rangle_{S_{0}}=\sum_{a_{1}, a_{2}, \ldots} C_{a 1, a 2, \ldots}^{n}\left\langle S_{i c o}^{1}\right\rangle_{S_{0}}^{a_{1}}\left\langle S_{i c o}^{2}\right\rangle_{S_{0}}^{a_{2}} \ldots \times \delta_{n}^{a_{1}+2 a_{2}+\ldots}
$$

onde a delta é inserida para fixar o grau do polinômio em $S_{i}$ no lado direito em $S_{i}^{n}$. O fator $C_{a 1, a 2, \ldots}^{n}$ é o número de maneiras distintas de formar o diagrama a partir das partes conexas, que pode ser contado como

$$
C_{a 1, a 2, \ldots}^{n}=\frac{\text { número de permut. dos } S_{i c o}^{n}}{\text { permut. internas }},
$$


onde as permutações internas são as permutações que não são relevantes. Estas permutações são contadas como

$$
\begin{aligned}
& \prod_{k}\left\{\left[\text { número de permut. de }\left\langle S_{i c o}^{k}\right\rangle \text { em }\left(\left\langle S_{i c o}^{k}\right\rangle^{a_{k}}\right)\right]\right. \\
& \left.\times\left[\text { número de permut. dos } S_{i c o} \operatorname{em~}\left\langle S_{i c o}^{k}\right\rangle\right]^{a_{k}}\right\} \\
& =\prod_{k} a_{k} !(k !)^{a_{k}}
\end{aligned}
$$

Substituindo em (3.64) obtemos

$$
\left\langle S_{i}^{n}\right\rangle_{S_{0}}=\sum_{a_{1}, a_{2}, \ldots} n ! \frac{\left\langle S_{i c o}^{1}\right\rangle_{S_{0}}^{a_{1}}}{a_{1} !} \frac{\left\langle S_{i c o}^{2}\right\rangle_{S_{0}}^{a_{2}}}{a_{2} !(2 !)^{a_{2}}} \ldots \frac{\left\langle S_{i c o}^{2}\right\rangle_{S_{0}}^{a_{k}}}{a_{k} !(k !)^{a_{k}}} \ldots \times \delta_{n}^{a_{1}+2 a_{2}+\ldots+k a_{k}+\ldots} .
$$

Calculando a soma soma sobre $n$ em 3.63 obtemos

$$
Z=Z_{0}\left\{\sum_{a_{1}, a_{2}, \ldots} \frac{\left\langle S_{i c o}^{1}\right\rangle_{S_{0}}^{a_{1}}}{a_{1} !} \frac{\left\langle S_{i c o}^{2}\right\rangle_{S_{0}}^{a_{2}}}{a_{2} !(2 !)^{a_{2}}} \ldots \frac{\left\langle S_{i c o}^{2}\right\rangle_{S_{0}}^{a_{k}}}{a_{k} !(k !)^{a_{k}}} \ldots\right\}
$$

Fatorando as somas independentes

$$
\begin{aligned}
Z & =Z_{0}\left\{\sum_{a_{1}=0}^{\infty} \frac{\left\langle S_{i c o}^{1}\right\rangle_{S_{0}}^{a_{1}}}{a_{1} !}\right\} \times\left\{\sum_{a_{2}=0}^{\infty} \frac{\left\langle S_{i \text { co }}^{2}\right\rangle_{S_{0}}^{a_{2}}}{a_{2} !(2 !)^{a_{2}}}\right\} \times \ldots \times\left\{\sum_{a_{k}=0}^{\infty} \frac{\left\langle S_{i \text { co }}^{k}\right\rangle_{S_{0}}^{a_{k}}}{a_{k} !(k !)^{a_{k}}}\right\} \ldots \\
& =Z_{0} \prod_{k=1}^{\infty} \exp \left[\frac{\left\langle S_{i c o}^{k}\right\rangle_{S_{0}}}{k !}\right]
\end{aligned}
$$

podemos escrever a função de partição como

$$
Z=Z_{0} \exp W
$$

onde $W=\log Z$ é escrito como

$$
W=\sum_{k=1}^{\infty} \frac{\left\langle S_{i c o}^{k}\right\rangle_{S_{0}}}{k !}
$$

Desta forma mostramos que $\log Z$ é uma soma de diagramas conexos. Em termos da pressão temos

$$
P=\frac{\log Z_{0}+W}{\beta V}
$$

de modo que a pressão pode ser escrita como diagramas conexos. 


$$
D_{0}\left(\omega_{n}, \vec{p}\right)
$$

Figura 3.1: Propagador do campo escalar real

\subsection{Regras de Feynman}

Nesta seção vamos definir as regras de Feynman para a teoria $\phi^{4}$ e veremos como os cálculos perturbativos são simplificados utilizando as técnicas diagramáticas. As regras de Feynman e os fatores combinatoriais seguem as convenções de tHV74.

A parte quadrática da lagrangiana tem a forma

$$
\mathcal{L}_{2}=-\frac{1}{2} \phi\left(\partial_{\mu} \partial^{\mu}+m^{2}\right) \phi
$$

e o propagador é o inverso do operador que aparece na parte quadrática no espaço dos momentos

$$
D_{0}(k)\left(-k^{2}+m^{2}\right)=1
$$

que implica em

$$
D_{0}\left(\omega_{k}, \vec{k}\right)=\frac{-1}{k^{2}-m^{2}}=\frac{1}{\omega_{k}^{2}+\vec{k}^{2}+m^{2}},
$$

que é o propagador do campo escalar livre (figura 3.1).

A parte de interação da ação é escrita como

$$
S_{i}^{(4)}=-\frac{\lambda}{4 !} \int_{0}^{\beta} d^{d} x \phi^{4}(\tau, \vec{x}) .
$$

Podemos passar para o espaço dos momentos obtendo

$$
\begin{aligned}
S_{i}^{(4)} & =-\lambda \beta^{2} V^{2} \sum_{n_{1}, \ldots, n_{4}} \int \frac{d^{d-1} k_{1}}{(2 \pi)^{d-1}} \cdots \frac{d^{d-1} k_{4}}{(2 \pi)^{d-1}} \beta \delta_{0}^{n_{1}+\cdots+n_{4}}(2 \pi)^{d-1} \delta^{(d-1)}\left(\vec{k}_{1}+\cdots+\vec{k}_{4}\right) \\
& \times \frac{\tilde{\phi}\left(\omega_{n_{1}}, \vec{k}_{1}\right) \tilde{\phi}\left(\omega_{n_{2}}, \vec{k}_{2}\right) \tilde{\phi}\left(\omega_{n_{3}}, \vec{k}_{3}\right) \tilde{\phi}\left(\omega_{n_{4}}, \vec{k}_{4}\right)}{4 !} .
\end{aligned}
$$

O vértice quártico (figura 3.2 ) é então definido como

$$
V^{(4)}=-\lambda
$$

onde foram omitidos o fator de simetria do vértice e as deltas de conservação de energia e momento. 


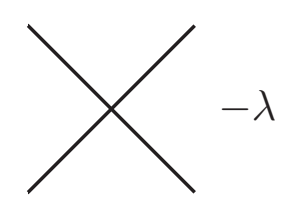

Figura 3.2: Vértice quártico

Seguindo as convenções de [tHV74], para o cálculo de contribuições para $\log Z_{n}$ em ordem $\lambda^{n}$ as regras de Feynman podem ser resumidas como

- Lembre que apenas diagramas conexos contribuem para $\log Z$ e colecione todas as topologias conexas nessa ordem de teoria de perturbação.

- Teremos um fator

$$
T \sum_{n_{k}} \int \frac{d^{d-1} k}{(2 \pi)^{d-1}} D_{0}\left(\omega_{k}, \vec{k}\right),
$$

para cada linha interna do diagrama e $k$ será o momento associado a esta linha.

- Para cada vértice estará associado um fator

$$
-\frac{1}{4 !} \lambda \beta \delta_{0}^{n_{1}+\cdots+n_{4}}(2 \pi)^{d-1} \delta\left(\vec{k}_{1}+\cdots+\vec{k}_{4}\right)
$$

que é o fator de vértice e a delta de conservação de momento no vértice.

- Conte de quantas maneiras diferentes podemos formar o digrama, que será o fator combinatorial (para mais detalhes sobre o teorema de Wick veja Gom02, por exemplo).

Para ilustrar vamos mostrar como essas regras de Feynman nos permite obter os mesmos resultados perturbativos obtidos no formalismo funcional.

\subsection{Primeira correção para a pressão}

Na seção 3.2 calculamos contribuições perturbativas para a função de partição usando diretamente o formalismo funcional. Usando as regras de Feynman definidas na seção anterior, veremos que é possível obter os mesmos resultados perturbativos, sem a necessidade dos longos cálculos de integrais funcionais.

Primeiramente, vamos calcular a contribuição para o logaritmo da função de partição em primeira ordem em $\lambda$. O diagrama que contribui para $\log Z$ nesta ordem esta repre- 


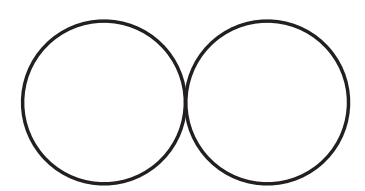

Figura 3.3: Contribuição ordem dois "loops" para $\log Z$

sentado na figura 3.3 . Usando as regras de Feynman da seção anterior, escrevemos essa contribuição como

$$
\log Z_{1}=-\frac{3 \lambda}{4 !} \sum_{n_{k}, n_{p}} T^{2} \int \frac{d^{d-1} k}{(2 \pi)^{d-1}} \frac{d^{d-1} p}{(2 \pi)^{d-1}} \beta \delta_{0}^{0}(2 \pi)^{d-1} \delta^{(d-1)}(0) D_{0}\left(\omega_{k}, \vec{k}\right) D_{0}\left(\omega_{p}, \vec{p}\right)
$$

de forma que as deltas são identicamente satisfeitas. A delta de momentos possui uma divergência tipo volume pois da definição da delta de Dirac escrevemos essa divergência como

$$
(2 \pi)^{d-1} \delta^{(d-1)}(0)=\int d^{d-1} x=V .
$$

Assim (3.81) possui um fator $\beta V$ e as integrais se fatoram, de forma que podemos reescrever 3.81) como

$$
\log Z_{1}=-\frac{3 \lambda}{4 !} \beta V\left[T \sum_{n_{k}} \int \frac{d^{d-1} k}{(2 \pi)^{d-1}} D_{0}\left(\omega_{k}, \vec{k}\right)\right]^{2}
$$

onde 3 é o fator combinatorial, que conta de quantas maneira diferentes podemos formar o diagrama. Assim vemos que o resultado é idêntico ao obtido no formalismo funcional (3.49). Para este termo temos que tratar uma única integral com a forma

$$
I=T \sum_{n_{k}} \int \frac{d^{d-1} k}{(2 \pi)^{d-1}} \frac{-1}{k^{2}-m^{2}}
$$

A soma de Matsubara pode ser transformada em uma integral no plano complexo, conforme mostrado no apêndice C, com a forma

$$
\begin{aligned}
I & =\int_{-i \infty+\epsilon}^{i \infty+\epsilon} \frac{d k_{0}}{2 \pi i} \int \frac{d^{d-1} k}{(2 \pi)^{d-1}}\left[1+2 N_{B}\left(k_{0}\right)\right] \frac{-1}{k_{0}^{2}-\vec{k}^{2}-m^{2}} \\
& =I_{0}+\int_{-i \infty+\epsilon}^{i \infty+\epsilon} \frac{d k_{0}}{2 \pi i} \int \frac{d^{d-1} k}{(2 \pi)^{d-1}} 2 N_{B}\left(k_{0}\right) \frac{-1}{k_{0}^{2}-\vec{k}^{2}-m^{2}}
\end{aligned}
$$


onde $I_{0}$ é a integral de temperatura zero

$$
\begin{aligned}
I_{0} & =\int_{-i \infty+\epsilon}^{i \infty+\epsilon} \frac{d k_{0}}{2 \pi i} \int \frac{d^{d-1} k}{(2 \pi)^{d-1}} \frac{-1}{k_{0}^{2}-\vec{k}^{2}-m^{2}} \\
& =\int \frac{d^{d} k_{E}}{(2 \pi)^{d}} \frac{1}{\left(k_{0}^{E}\right)^{2}+\vec{k}^{2}+m^{2}},
\end{aligned}
$$

com $k_{0}^{E}=-i k_{0}$ e $d^{d} k_{E}=d k_{0}^{E} d^{d-1} k$. Esta integral diverge quadraticamente em $d=4$ mas aqui está perfeitamente regularizada. Para o tratamento desta integrais em regularização dimensional veja, por exemplo, Gom02 .

A parte térmica da integral (3.85) pode ser tratada exatamente no limite de massa nula, que é o termo dominante no limite de altas temperaturas. Neste limite ela possui a forma

$$
I_{T}=\int_{-i \infty+\epsilon}^{i \infty+\epsilon} \frac{d k_{0}}{2 \pi i} \int \frac{d^{d-1} k}{(2 \pi)^{d-1}} 2 N_{B}\left(k_{0}\right) \frac{-1}{k_{0}^{2}-\vec{k}^{2}} .
$$

Fechando o contorno de integração no semiplano $R e\left(k_{0}\right)$ e usando o teorema dos resíduos obtemos

$$
\begin{aligned}
I_{T} & =\int \frac{d^{d-1} k}{(2 \pi)^{d-1}} \frac{N_{B}(|\vec{k}|)}{|\vec{k}|} \\
& =\frac{\Omega_{d-1} T^{d-2}}{(2 \pi)^{d-1}} \int_{0}^{\infty} d x x^{d-3} \frac{e^{-x}}{1-e^{-x}} \\
& =\frac{\Omega_{d-1} T^{d-2} \Gamma(d-2) \zeta(d-2)}{(2 \pi)^{d-1}}
\end{aligned}
$$

e substituindo o ângulo sólido em d dimensões teremos

$$
I_{T}=\frac{T^{d-2} \Gamma(d-2) \zeta(d-2)}{2^{d-2} \pi^{(d-1) / 2} \Gamma\left(\frac{d-1}{2}\right)},
$$

e em $d=4$ o resultado possui a forma [KG06]

$$
I_{T}=\frac{T^{2} \Gamma(2) \zeta(2)}{4 \pi^{3 / 2} \Gamma(3 / 2)}=\frac{T^{2}}{12} \quad(d=4),
$$

onde usamos $\Gamma(3 / 2)=1 / 2 \Gamma(1 / 2)=\sqrt{\pi} / 2$ e $\zeta(2)=\pi^{2} / 6$.

Portanto, substituindo 3.85 em 3.83 obtemos

$$
\log Z_{1}=-\frac{3 \lambda}{4 !} \beta V\left[I_{0}+I_{T}\right]^{2}
$$

Fazendo a renormalização de massa, vemos que o termos proporcionais a $I_{0}$ não contri- 
buirão para a pressão (veja a seção 3.7) [KG06], de modo que teremos

$$
\log Z_{1}^{r}=-\frac{3 \lambda}{4 !} \beta V\left[\frac{T^{d-2} \Gamma(d-2) \zeta(d-2)}{2^{d-2} \pi^{(d-1) / 2} \Gamma\left(\frac{d-1}{2}\right)}\right]^{2},
$$

e em quatro dimensões teremos a seguinte correção para a pressão

$$
P_{1}=\frac{\log Z_{1}^{r}}{\beta V}=-\frac{\lambda}{4 !} \frac{T^{4}}{48} \quad(d=4)
$$

\subsection{O propagador}

Explicitando o propagador na parte quadrática da ação no espaço dos momentos em (3.7), obtemos

$$
S_{0}=-\sum_{n} \int \frac{d^{d-1} p}{(2 \pi)^{d-1}} \frac{\beta^{2}}{2} D_{0}^{-1}\left(\omega_{n}, \vec{p}\right) \tilde{\phi}_{-n}(-\vec{p}) \tilde{\phi}_{n}(\vec{p})
$$

vemos que a função de correlação livre $\left\langle\tilde{\phi}_{-n}(-\vec{p}) \tilde{\phi}_{n}(\vec{p})\right\rangle_{S_{0}}$ possui a forma

$$
\begin{aligned}
\left\langle\tilde{\phi}_{-n}(-\vec{p}) \tilde{\phi}_{n}(\vec{p})\right\rangle_{S_{0}} & =-Z_{0}^{-1} \int \prod\left[d \phi_{l}(\vec{q})\right] \exp \left[\sum_{m} \int \frac{d^{d-1} k}{(2 \pi)^{d-1}} \frac{\beta^{2}}{2} D_{0}^{-1}\left(\omega_{m}, \vec{k}\right)\right. \\
& \left.\times \tilde{\phi}_{-m}(-\vec{k}) \tilde{\phi_{m}}(\vec{k})\right] \tilde{\phi}_{-n}(-\vec{p}) \tilde{\phi}_{n}(\vec{p}) .
\end{aligned}
$$

Assim, podemos escrever a correlação 3.95 como uma derivada funcional de $\log Z_{0}$ como

$$
\begin{aligned}
\left\langle\tilde{\phi}_{-n}(-\vec{p}) \tilde{\phi}_{n}(\vec{p})\right\rangle_{S_{0}} & =-\frac{2}{\beta^{2}} \frac{\delta}{\delta D_{0}^{-1}\left(\omega_{n}, \vec{p}\right)} \log Z_{0} \\
& =\frac{2}{\beta^{2}} D_{0}^{2}\left(\omega_{n}, \vec{p}\right) \frac{\delta}{\delta D_{0}\left(\omega_{n}, \vec{p}\right)} \log Z_{0},
\end{aligned}
$$

que é uma média térmica no ensemble descrito por $S_{0}$. Lembrando da expressão 3.12 para a função de partição livre, podemos reescreve-la como

$$
\log Z_{0}=-\frac{1}{2} \sum_{n} \sum_{\vec{p}} \log \left[\beta^{2} D_{0}^{-1}\left(\omega_{n}, \vec{p}\right)\right] .
$$

Calculando a derivada, obtemos para a função de correlação:

$$
\left\langle\tilde{\phi}_{-n}(-\vec{p}) \tilde{\phi}_{n}(\vec{p})\right\rangle_{S_{0}}=\frac{1}{\beta^{2}} D_{0}\left(\omega_{n}, \vec{p}\right)
$$

Isto nos motiva a definir o propagador completo como a correlação

$$
D\left(\omega_{n}, \vec{p}\right)=\beta^{2}\left\langle\tilde{\phi}_{-n}(-\vec{p}) \tilde{\phi}_{n}(\vec{p})\right\rangle_{S}
$$


que é uma média térmica no ensemble descrito por $S$. A auto-energia $\Pi\left(\omega_{n}, \vec{p}\right)$ é então definida em termos do propagador como

$$
D^{-1}\left(\omega_{n}, \vec{p}\right)=D_{0}^{-1}\left(\omega_{n}, \vec{p}\right)+\Pi\left(\omega_{n}, \vec{p}\right)
$$

que pode ser reescrito como

$$
\begin{aligned}
D\left(\omega_{n}, \vec{p}\right) & =\frac{1}{\omega_{n}^{2}+\vec{p}^{2}+m^{2}+\Pi} \\
& =\frac{1}{D_{0}^{-1}\left(\omega_{n}, \vec{p}\right)+\Pi\left(\omega_{n}, \vec{p}\right)} \\
& =\left(1+D_{0} \Pi\right)^{-1} D_{0} .
\end{aligned}
$$

A função de correlação de dois pontos para os campos na presença de interação pode ser escrita como

$$
\begin{aligned}
\left\langle\tilde{\phi}_{-n}(-\vec{p}) \tilde{\phi}_{n}(\vec{p})\right\rangle_{S} & =Z^{-1} \int \prod\left(d \phi_{l}(\vec{q})\right) \exp \left[\sum _ { m } \int \frac { d ^ { d - 1 } k } { ( 2 \pi ) ^ { d - 1 } } \left(-\frac{\beta^{2}}{2} D_{0}^{-1}\left(\omega_{m}, \vec{k}\right)\right.\right. \\
& \left.\left.\times \tilde{\phi}_{-m}(-\vec{k}) \tilde{\phi}_{m}(\vec{k})\right)+S_{i}\right] \tilde{\phi}_{-n}(-\vec{p}) \tilde{\phi}_{n}(\vec{p}),
\end{aligned}
$$

e pode ser reescrita como uma derivada funcional de $\log Z$ como

$$
\begin{aligned}
\left\langle\tilde{\phi}_{-n}(-\vec{p}) \tilde{\phi}_{n}(\vec{p})\right\rangle_{S} & =-\frac{2}{\beta^{2}} \frac{\delta}{\delta D_{0}^{-1}\left(\omega_{n}, \vec{p}\right)} \log Z \\
& =\frac{2}{\beta^{2}} D_{0}^{2}\left(\omega_{n}, \vec{p}\right) \frac{\delta}{\delta D_{0}\left(\omega_{n}, \vec{p}\right)} \log Z .
\end{aligned}
$$

Torna-se conveniente expandir em série de Taylor a expressão 3.101 como

$$
D\left(\omega_{n}, \vec{p}\right)=D_{0}-D_{0} \Pi D_{0}+D_{0} \Pi D_{0} \Pi D_{0}-D_{0} \Pi D_{0} \Pi D_{0} \Pi D_{0}+\ldots,
$$

e escrever $\Pi$ como uma expansão na constante de acoplamento $\lambda$

$$
\Pi=\Pi_{1}+\Pi_{2}+\Pi_{3} \ldots
$$

de modo que em primeira ordem teremos

$$
2 D_{0}^{2}\left(\omega_{n}, \vec{p}\right) \frac{\delta}{\delta D_{0}\left(\omega_{n}, \vec{p}\right)} \log Z_{1}=-D_{0}\left(\omega_{n}, \vec{p}\right) \Pi_{1}\left(\omega_{n}, \vec{p}\right) D_{0}\left(\omega_{n}, \vec{p}\right) .
$$

Portanto, substituindo (3.83) em (3.106), reescrevemos $\Pi_{1}$ como

$$
\Pi_{1}=2 \frac{\delta}{\delta D_{0}\left(\omega_{n}, \vec{p}\right)} \frac{\lambda}{8} \beta V\left[\sum_{n_{k}} T \int \frac{d^{d-1} k}{(2 \pi)^{d-1}} D_{0}\left(\omega_{k}, \vec{k}\right)\right]^{2} .
$$




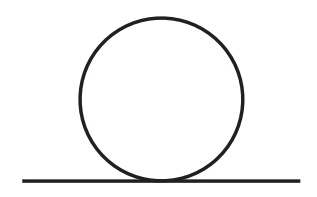

Figura 3.4: Contribuição de primeira ordem para a auto energia

Para o cálculo da derivada funcional é conveniente escrever 3.107 no caso discreto como

$$
\frac{\delta}{\delta D_{0}\left(\omega_{n}, \vec{p}\right)} \sum_{n_{k}} T \int \frac{d^{d-1} k}{(2 \pi)^{d-1}} D_{0}\left(\omega_{k}, \vec{k}\right) \rightarrow \frac{\delta}{\delta D_{0}\left(\omega_{n}, \vec{p}\right)} \sum_{n_{k}, \vec{k}} \frac{T}{V} D_{0}\left(\omega_{k}, \vec{k}\right)=\frac{T}{V},
$$

O que nos permite escrever escrever $\Pi_{1}$ como

$$
\Pi_{1}=\frac{\lambda}{2} \sum_{n_{k}} T \int \frac{d^{d-1} k}{(2 \pi)^{d-1}} D_{0}\left(\omega_{k}, \vec{k}\right)
$$

Representamos $\Pi_{1}$ como no diagrama da figura 3.4. Note que não inserimos os propagadores externos nas formas analíticas de diagramas com linhas externas.

Usando 3.104 juntamente com 3.103 vemos que em segunda ordem teremos

$$
\begin{aligned}
& 2 D_{0}^{2}\left(\omega_{n}, \vec{p}\right) \frac{\delta}{\delta D_{0}\left(\omega_{n}, \vec{p}\right)} \log Z_{2}=-D_{0}\left(\omega_{n}, \vec{p}\right) \Pi_{2}\left(\omega_{n}, \vec{p}\right) D_{0}\left(\omega_{n}, \vec{p}\right) \\
& +D_{0}\left(\omega_{n}, \vec{p}\right) \Pi_{1}\left(\omega_{n}, \vec{p}\right) D_{0}\left(\omega_{n}, \vec{p}\right) \Pi_{1}\left(\omega_{n}, \vec{p}\right) D_{0}\left(\omega_{n}, \vec{p}\right) .
\end{aligned}
$$

Calculando a derivada funcional da expressão 3.62 utilizando 3.108 obtemos

$$
\begin{aligned}
\frac{\delta}{\delta D_{0}\left(\omega_{n}, \vec{p}\right)} \log Z_{2} & =4 \frac{\lambda^{2}}{48} \sum_{n_{k}, n_{q}} T^{2} \int \frac{d^{d-1} k}{(2 \pi)^{d-1}} \frac{d^{d-1} q}{(2 \pi)^{d-1}} \\
& \times D_{0}\left(\omega_{k}, \vec{k}\right) D_{0}\left(\omega_{q}, \vec{q}\right) D_{0}\left(\omega_{p}+\omega_{k}+\omega_{q}, \vec{p}+\vec{k}+\vec{q}\right) \\
& +2 \frac{\lambda^{2}}{16}\left[\sum_{n_{k}} T \int \frac{d^{d-1} k}{(2 \pi)^{d-1}} D_{0}\left(\omega_{k}, \vec{k}\right)\right]^{2} D_{0}\left(\omega_{p}, \vec{p}\right) \\
& +2 \frac{\lambda^{2}}{16} \sum_{n_{k}} T \int \frac{d^{d-1} k}{(2 \pi)^{d-1}} D_{0}\left(\omega_{k}, \vec{k}\right) \sum_{n_{l}} \int \frac{d^{d-1} l}{(2 \pi)^{d-1}}\left[D_{0}\left(\omega_{l}, \vec{l}\right)\right]^{2}
\end{aligned}
$$

Lembrando a expressão para $\Pi_{1}$ (3.107) escrevemos para parte redutível (figura 3.5 de 3.110 como

$$
\Pi_{1}\left(\omega_{n}, \vec{p}\right) D_{0}\left(\omega_{n}, \vec{p}\right) \Pi_{1}\left(\omega_{n}, \vec{p}\right)=\frac{\lambda^{2}}{4}\left[\sum_{n_{k}} T \int \frac{d^{d-1} k}{(2 \pi)^{d-1}} D_{0}\left(\omega_{k}, \vec{k}\right)\right]^{2} D_{0}\left(\omega_{p}, \vec{p}\right) .
$$




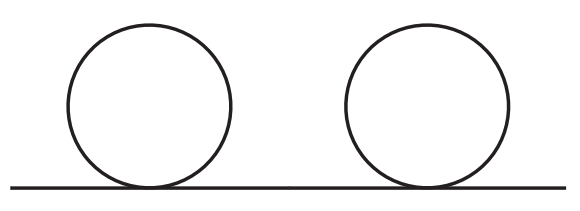

Figura 3.5: Contribuição redutível para o propagador.
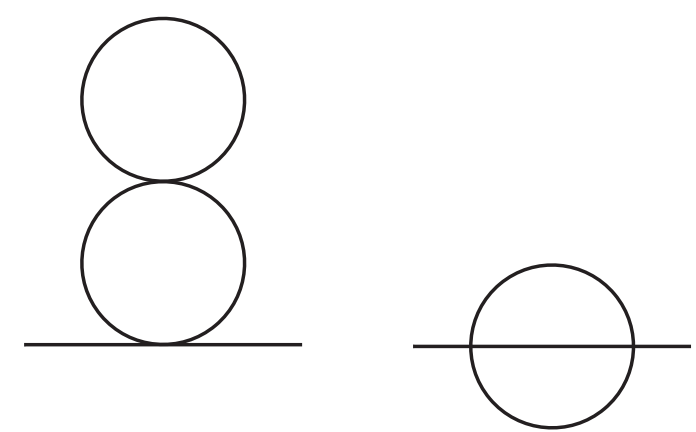

Figura 3.6: Auto-energia em ordem dois laços $\Pi_{2}$.

Desta forma, a parte redutíve $]^{1}$ de 3.110 se cancela e $\Pi_{2}$ pode ser escrito como

$$
\begin{aligned}
\Pi_{2}\left(\omega_{p}, \vec{p}\right) & =-\frac{\lambda^{2}}{6} \sum_{n_{k}, n_{q}} T^{2} \int \frac{d^{d-1} k}{(2 \pi)^{d-1}} \frac{d^{d-1} k}{(2 \pi)^{d-1}} \\
& \times D_{0}\left(\omega_{k}, \vec{k}\right) D_{0}\left(\omega_{q}, \vec{q}\right) D_{0}\left(\omega_{p}+\omega_{k}+\omega_{q}, \vec{p}+\vec{k}+\vec{q}\right) \\
& -\frac{\lambda^{2}}{4} \sum_{n_{k}} T \int \frac{d^{d-1} k}{(2 \pi)^{d-1}} D_{0}\left(\omega_{k}, \vec{k}\right) \\
& \times \sum_{n_{l}} \int \frac{d^{d-1} l}{(2 \pi)^{d-1}} D_{0}^{2}\left(\omega_{l}, \vec{l}\right) .
\end{aligned}
$$

A representação diagramática de $\Pi_{2}$ é mostrada na figura 3.6 .

Estabeleceremos agora que apenas diagramas irredutíveis contribuem para a auto energia a dois laços. Podemos mostrar agora que em geral $\Pi_{n}$ é uma soma de diagramas irredutíveis. Para isto vamos escrever a soma de todas as contribuições irredutíveis para o propagador como

$$
D_{I R}\left(\omega_{p}, \vec{p}\right)=-D_{0}\left(\omega_{p}, \vec{p}\right) \Pi_{I R}\left(\omega_{p}, \vec{p}\right) D_{0}\left(\omega_{p}, \vec{p}\right)
$$

A contribuição geral redutível para o propagador com $n$ partes irredutíveis será dada por (figura 3.7 )

$$
D_{n R}\left(\omega_{p}, \vec{p}\right)=\frac{C_{n}}{2^{n} n !}\left\{D_{0}\left(\omega_{p}, \vec{p}\right)\left[-\Pi_{I R}\left(\omega_{p}, \vec{p}\right)\right]\right\}^{n} D_{0}\left(\omega_{p}, \vec{p}\right) .
$$

\footnotetext{
${ }^{1} \mathrm{Um}$ diagrama é dito irredutível ou 1PI (usaremos a sigla em inglês "One Particle Irreducible") se não podemos separa-lo em dois diagramas cortando apenas uma linha interna. Caso contrário dizemos que o diagrama é redutível.
} 


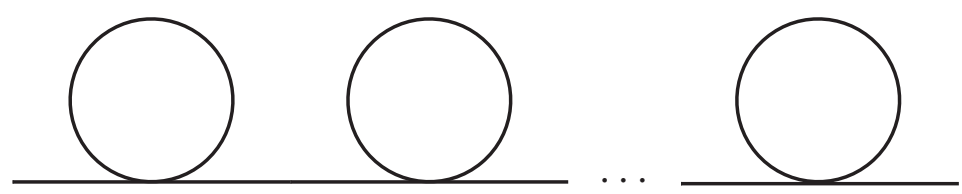

Figura 3.7: Contribuição redutível com $n$ inserções da auto energia

Onde $C_{n}$ é o fator combinatorial, $1 / n$ ! decorre da indistinguibilidade dos $\Pi_{I R}$ e $1 / 2^{n}$ é um fator de simetria das pernas externas de cada um dos $\Pi_{I R}$. O sinal - decorre do fato que $\Pi$ é dado pelo negativo do diagrama de Feynman correspondente. O fator combinatorial é dado por $n$ ! devido a permutação dos $\Pi$, vezes $2^{n}$ da troca dos propagadores externos a cada um dos $\Pi$. Deste modo a contribuição redutível com $n$ partes irredutíveis para o propagador será escrita como

$$
D_{n R}\left(\omega_{p}, \vec{p}\right)=\left[-D_{0}\left(\omega_{p}, \vec{p}\right) \Pi_{I R}\left(\omega_{p}, \vec{p}\right)\right]^{n} D_{0}\left(\omega_{p}, \vec{p}\right)
$$

Assim o propagador completo é escrito como

$$
D\left(\omega_{p}, \vec{p}\right)=\sum_{n=0}^{\infty}\left[-D_{0}\left(\omega_{p}, \vec{p}\right) \Pi_{I R}\left(\omega_{p}, \vec{p}\right)\right]^{n} D_{0}\left(\omega_{p}, \vec{p}\right) .
$$

A equação (3.104) juntamente com (3.117) nos permitem escrever

$$
\Pi_{I R}=\Pi
$$

ou seja, apenas diagramas irredutíveis contribuem para $\Pi$.

\subsection{A auto-energia}

A contribuição de primeira ordem para П é dada por 3.109

$$
\Pi_{1}=\frac{\lambda}{2} \sum_{n_{k}} T \int \frac{d^{d-1} k}{(2 \pi)^{d-1}} \frac{1}{\omega_{k}^{2}+\vec{k}^{2}+m^{2}} .
$$

A integral que aparece na auto-energia é da forma (3.84). Portanto, no caso de massa nula, usando 3.85 e 3.89 teremos

$$
\begin{aligned}
\Pi_{1} & =\frac{\lambda}{2} I_{0}+\lambda T^{d-2} \frac{\Gamma(d-2) \zeta(d-2)}{2^{d-1} \pi^{(d-1) / 2} \Gamma\left(\frac{d-1}{2}\right)} \\
& =\Pi_{0}+\Pi_{T},
\end{aligned}
$$




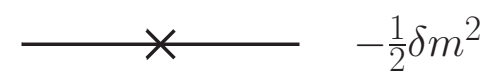

Figura 3.8: Vértice referente ao contra-termo de massa

com a parte térmica sendo

$$
\Pi_{T}=\lambda T^{d-2} \frac{\Gamma(d-2) \zeta(d-2)}{2^{d-1} \pi^{(d-1) / 2} \Gamma\left(\frac{d-1}{2}\right)},
$$

e em $d=4$ temos [KG06]

$$
\Pi_{T}=\frac{\lambda}{4 !} T^{2}(d=4)
$$

A forma explícita da parte de temperatura zero, que pode ser obtida de (3.86), possui a forma

$$
\Pi_{0}=\frac{\lambda}{2} \int \frac{d^{d} k_{E}}{(2 \pi)^{d}} \frac{1}{\left(k_{0}^{E}\right)^{2}+\vec{k}^{2}},
$$

e não será tratada neste trabalho ( para um tratamento de integrais em regularização dimensional veja Gom02, por exemplo). Este termo de temperatura zero apresenta uma divergência quadrática em quatro dimensões do espaço tempo, de forma que a massa precisa ser renormalizada. Introduzimos então um contra-termo na lagrangiana com a forma

$$
\mathcal{L}_{\delta m^{2}}=-\delta m^{2} \phi^{2}
$$

e reescrevemos a lagrangiana (3.1) como

$$
\begin{aligned}
\mathcal{L}_{r} & =\mathcal{L}+\mathcal{L}_{\delta m^{2}} \\
& =\frac{1}{2} \partial_{\mu} \phi \partial^{\mu} \phi-\frac{1}{2}\left(m^{2}\right) \phi^{2}-V(\phi)-\frac{1}{2} \delta m^{2} \phi^{2},
\end{aligned}
$$

onde o último termo é tratado como uma perturbação, com a regra de Feynman mostrada na figura 3.8. A parte de temperatura zero da auto-energia não pode mudar o polo do propagador (3.101) de forma que vamos impor

$$
\delta m^{2}+\Pi_{0}=0
$$




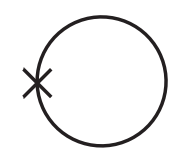

Figura 3.9: Contribuição do contra-termo de massa para $\log Z$.

de forma que o propagador completo da teoria fica livre de divergências. Deste modo chegamos na forma explícita para o contra-termo de massa, em ordem $\lambda$, como

$$
\delta m^{2}=-\frac{\lambda}{2} I_{0}
$$

Este contra-termo irá contribuir para o $\log (Z)$ a um laço. Usando (3.39), veremos que a contribuição em ordem $\lambda$, que está representada na figura 3.9 , possui a forma

$$
\log Z_{1}^{\delta m^{2}}=-\frac{1}{2} \delta m^{2}\left\langle\int_{0}^{\beta} d^{d} x \phi^{2}\right\rangle_{S_{0}}
$$

Na representação de Fourier podemos escrever

$$
\left\langle\int_{0}^{\beta} d^{d} x \phi^{2}\right\rangle_{S_{0}}=\sum_{n} V \int \frac{d^{d-1} p}{(2 \pi)^{d-1}} \beta^{2}\left\langle\tilde{\phi}_{-n}(-\vec{p}) \tilde{\phi}_{n}(\vec{p})\right\rangle_{S_{0}},
$$

e identificando o propagador 3.98 , obtemos

$$
\begin{aligned}
\log Z_{1}^{\delta m^{2}} & =-\frac{1}{2} \beta V \delta m^{2} T \sum_{n} \int \frac{d^{d-1} p}{(2 \pi)^{d-1}} \cdot D_{0}\left(\omega_{n}, \vec{p}\right) \\
& =-\frac{1}{2} \beta V \delta m^{2}\left(I_{0}+I_{T}\right) \\
& =\frac{\lambda}{4} \beta V\left(I_{0}^{2}+I_{0} I_{T}\right) .
\end{aligned}
$$

Portanto, juntando com o resultado de primeira ordem em (3.91), obtemos o resultado renormalizado

$$
\log Z_{1}^{r}=\log Z_{1}+\log Z_{1}^{\delta m^{2}}=-\frac{\lambda}{8} \beta V\left(I_{T}^{2}-I_{0}^{2}\right)
$$

nesta expressão, o termo de proporcional a $I_{0}^{2}$ contribuirá como uma constante independente da temperatura na pressão e será desprezado. Deste modo obtemos o resultado renormalizado dado por (3.92).

Este procedimento nos ensina uma lição. Para obter a pressão em uma dada ordem em $\lambda$ termos que renormalizar a teoria até a mesma ordem. Não foi preciso renormalizar a função de quatro pontos por que a primeira correção para lambda introduzira um contra-termo de ordem $\lambda^{2}$ que só entrará no calculo da pressão em ordem de três 
laços. Entretanto, a equação 3.126 mostra que renormalização da função de dois pontos é, em geral, trivial. Assim, podemos simplesmente ignorar os termos proporcionais a auto-energia a temperatura zero nas contribuições $\log Z$ em ordem $\lambda$, tal como feito na seção 3.5. sem perder nenhuma contribuição. Entretanto, a partir da ordem $\lambda^{2}$ teremos que renormalizar a teoria de maneira sistemática e, além disso, introduzir a lagrangiana efetiva na região dos "Hard Thermal Loops" para controlar as divergências infravermelhas [FST92, AZ94, AxZ95].

\subsection{Soma das divergências infravermelhas}

A presença de divergências infravermelhas faz com que a série perturbativa usual perca sentido, sendo necessário um procedimento de resoma, que consiste em um reordenamento em alguns termos da série perturbativa. Neste procedimento de resoma, consideramos uma série infinita de diagramas que, ordem à ordem, são dominantes no infravermelho. Embora o resultado deste procedimento seja finito no infravermelho, ele é não analítico na constante de acoplamento [KG06, Bel96]. Nesta seção veremos que o comportamento não analítico da série perturbativa depende qualitativamente da paridade da dimensão do espaço-tempo $d$ : quando $d$ é par a não-analiticidade é da forma $\lambda^{(d-1) / 2}$, enquanto para $d$ ímpar a não-analiticidade é apenas logarítmica [BFS12].

Como vimos, quando a massa é nula, a contribuição em ordem $\lambda^{2}$ para o logaritmo da função de partição dada é por (3.62). Usando o propagador de massa nula

$$
D_{0}\left(\omega_{k}, \vec{k}\right)=\frac{1}{\omega_{k}^{2}+\vec{k}^{2}}
$$

esta contribuição pode ser expressa na forma

$$
\begin{aligned}
\log Z_{2} & =\frac{\lambda^{2}}{48} \beta V \sum_{n_{k}, n_{p}, n_{l}} T^{3} \int \frac{d^{d-1} k}{(2 \pi)^{d-1}} \frac{d^{d-1} p}{(2 \pi)^{d-1}} \frac{d^{d-1} l}{(2 \pi)^{d-1}} \\
& \times D_{0}\left(\omega_{k}, \vec{k}\right) D_{0}\left(\omega_{p}, \vec{p}\right) D_{0}\left(\omega_{l}, \vec{l}\right) D_{0}\left(\omega_{k}+\omega_{p}+\omega_{l}, \vec{k}+\vec{p}+\vec{l}\right) \\
& +\frac{\lambda^{2}}{16} \beta V\left[\sum_{n_{k}} T \int \frac{d^{d-1} k}{(2 \pi)^{d-1}} D_{0}\left(\omega_{k}, \vec{k}\right)\right]^{2} \sum_{n_{l}} T \int \frac{d^{d-1} l}{(2 \pi)^{d-1}} D_{0}^{2}\left(\omega_{l}, \vec{l}\right) .
\end{aligned}
$$

O segundo termo da equação 3.133), que corresponde ao diagrama da figura 3.10 , pode ser reescrito como

$$
\frac{\beta V}{4} \Pi_{T}^{2} T \sum_{n_{q}} \int \frac{d^{d-1} q}{(2 \pi)^{d-1}}\left[\frac{1}{\omega_{q}^{2}+\vec{q}^{2}}\right]^{2}
$$




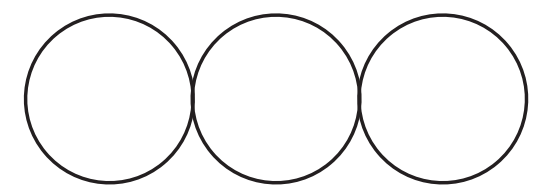

Figura 3.10: Contribuição ordem três laços para $\log Z$.

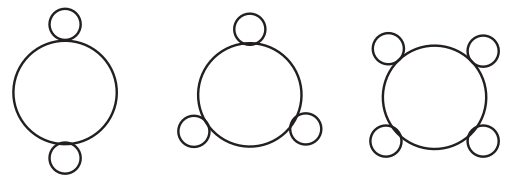

Figura 3.11: Diagramas anelares

onde estamos supondo que fizemos a renormalização de massa, tal como na seção anterior, de tal maneira que apenas a parte térmica da auto energia irá contribuir. Em quatro dimensões, o modo estático da soma de Matsubara $n_{q}=0$ possui um comportamento linearmente divergente no infravermelho $(\vec{q} \rightarrow 0)$. Desta forma a parte dominante no infravermelho de 3.133 é dada pelo diagrama da figura 3.10 .

Em geral [KG06, Bel96], em uma dada ordem em $\lambda$, os diagramas dominantes no infravermelho são os diagramas formados por inserções sucessivas da auto energia $\Pi_{T}$ tais como os diagramas mostrados na figura 3.11. Note que o grau de divergência é aumentado a cada inserção de $\Pi_{T}$.

Cientes disto, vamos obter a expressão analítica de um particular diagrama mostrado na figura 3.12, que chamaremos de diagrama anelar, em ordem $N+1$ laços $(N$ inserções de $\left.\Pi_{1}\right)$. O fator combinatorial é calculado da seguinte forma: temos um fator $(N-1) ! / 2$ de ordenar as $N$ inserções de $\Pi_{T}$ em um círculo e um fator $2^{N}$ de trocar as duas linhas externas de cada $\Pi_{T}$. Temos que dividir por um fator $N$ ! da indistinguibilidade dos $\Pi_{T}$ e $2^{N}$ da simetria de $\Pi_{T}$. Levando tudo isto em conta, o fator combinatorial deste diagrama é $1 /(2 N)$. Desta forma, escrevemos a expressão analítica deste diagrama como

$$
\frac{\beta V}{2} \sum_{n} T \int \frac{d^{d-1} p}{(2 \pi)^{d-1}} \frac{\left(-D_{0}\left(\omega_{n}, \vec{p}\right) \Pi_{T}\right)^{N}}{N},
$$

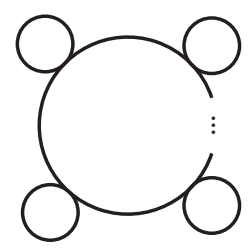

Figura 3.12: Diagrama anelar de ordem $N+1$ laços 
e o sinal negativo vem da mesma razão que os sinal negativo em (3.115), ou seja, a auto energia é dada pelo negativo do diagrama de Feynman correspondente. O modo estático deste diagrama possui a forma

$$
R_{N}=\frac{V}{2} \int \frac{d^{d-1} p}{(2 \pi)^{d-1}} \frac{1}{N}\left[-\frac{\Pi_{T}}{\vec{p}^{2}}\right]^{N}
$$

Por contagem de potências, temos que esta quantidade é divergente no infravermelho para $N \geq E(d / 2)$, onde $E(d / 2)$ é a parte inteira de $d / 2$, isto é

$$
E\left(\frac{d}{2}\right)=\left\{\begin{array}{lll}
\frac{d}{2}, & d & \text { par } \\
\frac{d-1}{2}, & d & \text { impar. }
\end{array}\right.
$$

Deste modo, somando sobre os termos dominantes no infravermelho em cada ordem em $\lambda$, obtemos

$$
\log Z_{\text {rings }}=\frac{V}{2} \sum_{N=E(d / 2)}^{\infty} \int \frac{d^{d-1} p}{(2 \pi)^{d-1}} \frac{1}{N}\left[-\frac{\Pi_{T}}{\vec{p}^{2}}\right]^{N}
$$

Fatorando a integração nos ângulos, e fazendo uma mudança de variáveis

$$
x=\frac{|\vec{p}|}{\Pi_{T}^{1 / 2}}
$$

podemos reescrever esta contribuição como

$$
\log Z_{\text {rings }}=\frac{V \Pi_{T}^{(d-1) / 2} \Omega_{d-1}}{(2 \pi)^{d-1}} \sum_{N=E(d / 2)}^{\infty} \int_{0}^{\infty} d x(-1)^{N} x^{d-2} \frac{1}{2 N x^{2 N}} .
$$

Neste ponto é conveniente separar nosso estudo em dois casos.

\subsubsection{Caso $d$ par}

Neste caso a equação 3.140 tem a forma

$$
\log Z_{\text {rings }}^{d \text { par }}=\frac{V \Pi_{T}^{(d-1) / 2} \Omega_{d-1}}{(2 \pi)^{d-1}} \sum_{N=d / 2}^{\infty} \int_{0}^{\infty} d x(-1)^{N} x^{d-2} \frac{1}{2 N x^{2 N}} .
$$

Fazendo uma integração por partes na integral na integral resultante teremos

$$
\sum_{N=d / 2}^{\infty} \int_{0}^{\infty} d x(-1)^{N} x^{d-2} \frac{1}{2 N x^{2 N}}=\sum_{N=d / 2}^{\infty}\left\{\left.\frac{x^{d-1}}{d-1} \frac{(-1)^{N}}{2 N x^{2 N}}\right|_{0} ^{\infty}+\int_{0}^{\infty} d x \frac{x^{d-1}}{d-1} \frac{(-1)^{N}}{x^{2 N+1}}\right\}
$$


Neste caso, o termo de superfície se anula. Para ver isso vamos computar os limites. Primeiro $x$ tendendo a infinito é fácil de perceber que o limite se anula por simples contagem de potências, obtendo

$$
\sum_{N=d / 2}^{\infty} \frac{x^{d-1}}{d-1} \frac{(-1)^{N}}{2 N x^{2 N}} \longrightarrow 0 \quad(x \rightarrow \infty) .
$$

Já para $x$ tendendo a zero, é conveniente reescrever

$$
\sum_{N=d / 2}^{\infty} \frac{x^{d-1}}{d-1} \frac{(-1)^{N}}{2 N x^{2 N}}=-\frac{x^{d-1}}{2(d-1)} \log \left(1+\frac{1}{x^{2}}\right)-\frac{x^{d-1}}{2(d-1)} \sum_{N=1}^{d / 2-1} \frac{(-1)^{N}}{N x^{2 N}}
$$

O segundo termo se anula trivialmente para $x \rightarrow 0$, enquanto o primeiro termo apresenta uma indeterminação no limite. No entanto, podemos usar a regra de L'Hôpital para ver que o limite se anula, de modo que teremos

$$
\sum_{N=d / 2}^{\infty} \frac{x^{d-1}}{d-1} \frac{(-1)^{N}}{2 N x^{2 N}} \longrightarrow 0 \quad(x \rightarrow 0)
$$

o que implica que o termo de superfície da equação 3.142 é nulo.

Resta ainda resolver uma integral na equação 3.142. Para tanto, vamos comutar a soma com a integral para reescreve

$$
\begin{aligned}
\sum_{N=d / 2}^{\infty} \int_{0}^{\infty} d x(-1)^{N} x^{d-2} \frac{1}{2 N x^{2 N}} & =\sum_{N=d / 2}^{\infty} \int_{0}^{\infty} d x \frac{x^{d-1}}{d-1} \frac{(-1)^{N}}{x^{2 N+1}} \\
& =\frac{1}{d-1} \int_{0}^{\infty} d x \frac{1}{x^{2}} \sum_{N=d / 2}^{\infty} \frac{(-1)^{N}}{x^{2(N-d / 2)}} \\
& =\frac{(-1)^{d / 2}}{d-1} \int_{0}^{\infty} d x \frac{1}{x^{2}} \sum_{N=0}^{\infty} \frac{(-1)^{N}}{x^{2 N}} \\
& =\frac{(-1)^{d / 2}}{d-1} \int_{0}^{\infty} d x \frac{1}{1+x^{2}} \\
& =\frac{(-1)^{d / 2}}{d-1} \frac{1}{2} \int_{-\infty}^{\infty} d x \frac{1}{1+x^{2}} \\
& =\frac{(-1)^{d / 2} \pi}{2(d-1)}
\end{aligned}
$$

de modo que teremos

$$
\log Z_{\text {rings }}^{d \text { par }}=\frac{V \Pi_{T}^{(d-1) / 2} \Omega_{d-1}}{(2 \pi)^{d-1}} \frac{(-1)^{d / 2} \pi}{2(d-1)}=\frac{V(-1)^{d / 2} \Pi_{T}{ }^{(d-1) / 2}}{2^{d} \pi^{(d-3) / 2} \Gamma\left(\frac{d+1}{2}\right)},
$$

que exibe um comportamento não analítico tipo $\lambda^{(d-1) / 2}$ na constante de acoplamento. A 
pressão pode ser escrita como

$$
P_{\text {rings }}^{d \text { par }}=\frac{T(-1)^{d / 2} \Pi_{T}^{(d-1) / 2}}{2^{d} \pi^{(d-3) / 2} \Gamma\left(\frac{d+1}{2}\right)} .
$$

Em particular, em um espaço-tempo de 4 dimensões, usando o resultado da auto-energia 3.122 obtemos

$$
P_{\text {rings }}^{d=4}=\left(\frac{\lambda}{4 !}\right)^{3 / 2} \frac{T^{4}}{12 \pi},
$$

e assim, juntando os resultados (3.31), 3.93 e 3.149 obtemos a pressão até ordem $\lambda^{3 / 2}$ com a forma [KG06]

$$
P=\frac{T^{4} \pi^{2}}{90}-\frac{\lambda}{4 !} \frac{T^{4}}{48}+\left(\frac{\lambda}{4 !}\right)^{3 / 2} \frac{T^{4}}{12 \pi}
$$

\subsubsection{Caso $d$ impar}

No caso $d$ impar a soma 3.140 possui a forma

$$
\log Z_{\text {rings }}^{\text {dimpar }}=\frac{V \Pi_{T}^{(d-1) / 2} \Omega_{d-1}}{(2 \pi)^{d-1}} \sum_{N=(d-1) / 2}^{\infty} \int_{0}^{\infty} d x(-1)^{N} x^{d-2} \frac{1}{2 N x^{2 N}} .
$$

Fazendo também neste caso uma integração por partes teremos

$$
\begin{aligned}
\sum_{N=(d-1) / 2}^{\infty} \int_{0}^{\infty} d x(-1)^{N} x^{d-2} \frac{1}{2 N x^{2 N}} & =\left.\sum_{N=(d-1) / 2}^{\infty} \frac{x^{d-1}}{d-1} \frac{(-1)^{N}}{2 N x^{2 N}}\right|_{0} ^{\infty} \\
& +\sum_{N=(d-1) / 2}^{\infty} \int_{0}^{\infty} d x \frac{x^{d-1}}{d-1} \frac{(-1)^{N}}{x^{2 N+1}}
\end{aligned}
$$

mas aqui o termo de superfície é não nulo, pois no limite para $x$ tendendo ao infinito segue que

$$
\sum_{N=(d-1) / 2}^{\infty} \frac{x^{d-1}}{d-1} \frac{(-1)^{N}}{2 N x^{2 N}} \longrightarrow \frac{(-1)^{(d-1) / 2}}{(d-1)^{2}} \quad x \rightarrow \infty .
$$

Usando um argumento semelhante ao usado no caso $d$ par, vemos que para $x \rightarrow 0$ vemos que o limite se anula

$$
\begin{aligned}
\sum_{N=(d-1) / 2}^{\infty} \frac{x^{d-1}}{d-1} \frac{(-1)^{N}}{2 N x^{2 N}} & =-\frac{x^{d-1}}{2(d-1)} \log \left(1+\frac{1}{x^{2}}\right)-\frac{x^{d-1}}{2(d-1)} \sum_{N=1}^{(d-1) / 2-1} \frac{(-1)^{N}}{N x^{2 N}} \\
& \longrightarrow 0 \quad x \rightarrow 0,
\end{aligned}
$$


de modo que o termo de superfície de (3.152) é dado pela equação (3.153). Assim, substituindo (3.153) em 3.152), e seguindo passos semelhantes aos usado em (3.146), podemos ver que

$$
\begin{aligned}
\sum_{N=(d-1) / 2}^{\infty} \int_{0}^{\infty} d x(-1)^{N} x^{d-2} \frac{1}{2 N x^{2 N}} & =\frac{(-1)^{(d-1) / 2}}{(d-1)^{2}}+\sum_{N=(d-1) / 2}^{\infty} \int_{0}^{\infty} d x \frac{x^{d-1}}{d-1} \frac{(-1)^{N}}{x^{2 N+1}} \\
& =\frac{(-1)^{(d-1) / 2}}{(d-1)^{2}}+\frac{(-1)^{(d-1) / 2}}{(d-1)} \int_{0}^{\infty} d x \frac{x}{1+x^{2}}
\end{aligned}
$$

A integral resultante é logaritmicamente divergente no ultravioleta 2 , Para trata-la, introduzimos uma escala corte $\mu$ na integral no momento. Portanto, lembrando da mudança de variáveis 3.139 teremos

$$
\sum_{N=(d-1) / 2}^{\infty} \int_{0}^{\mu / \Pi_{T}^{1 / 2}} d x(-1)^{N} x^{d-2} \frac{1}{2 N x^{2 N}}=\frac{(-1)^{(d-1) / 2}}{(d-1)^{2}}+\frac{(-1)^{(d-1) / 2}}{2(d-1)} \log \left(1+\frac{\mu^{2}}{\Pi_{T}}\right),
$$

e substituindo na equação (3.151), obtemos

$$
\log Z_{\text {rings }}^{d \text { par }}=\frac{V(-1)^{(d-1) / 2} \Pi_{T}^{(d-1) / 2}}{2^{d} \pi^{(d-3) / 2} \Gamma\left(\frac{d+1}{2}\right)}\left[\frac{2}{d-1}+\log \left(1+\frac{\mu^{2}}{\Pi_{T}}\right)\right]
$$

que possui um comportamento não analítico tipo logarítmico na constante de acoplamento. A escala $\mu$ é, em princípio, arbitrária. Entretanto, no limite de altas temperaturas, esperamos que $\mu$ seja da ordem da temperatura.

\footnotetext{
${ }^{2}$ Essa divergência é esperada pois a série (3.151) começa com um termo logaritmicamente divergente, tanto no ultravioleta, quanto no infravermelho.
} 


\section{Eletrodinâmica Quântica a Temperatura Finita em d dimensões}

Neste capítulo estudaremos a Eletrodinâmica Quântica (QED)1a temperatura finita em um espaço-tempo d-dimensional no formalismo do tempo imaginário. Os resultados obtidos neste capítulo são aplicáveis a regimes físicos em que a temperatura é elevada em relação a quaisquer outras escalas de massa do problema. De maneira análoga ao que ocorre com o campo escalar, veremos que as divergências infravermelhas presentes em diagramas que contribuem para pressão introduzem uma não-analiticidade na série perturbativa, e ainda veremos que este comportamento não-analítico depende da paridade da dimensão do espaço-tempo. Se a dimensão do espaço-tempo $d$ for par, a nãoanaliticidade se da na forma $\left(e^{2}\right)^{(d-1) / 2}$, enquanto se $d$ for ímpar, a não-analiticidade é apenas logarítmica [BFS12].

\subsection{A função de partição da Eletrodinâmica Quântica}

Combinando os termos de Dirac e de campos de calibre obtidos no capítulo 2.2 (veja as seções 2.2.3 e 2.2.5), a lagrangiana total da QED, já fixado o calibre de Feynman 2 $\xi=1$, juntamente com os fantasmas de Faddeev-Popov é dada por

$$
\mathcal{L}=-\frac{1}{4}\left(\partial_{\mu} A_{\nu}-\partial_{\nu} A_{\mu}\right)^{2}+i \bar{\psi} \gamma^{\mu}\left(\partial_{\mu}-i e A_{\mu}\right) \psi-\frac{1}{2}\left(\partial_{\mu} A^{\mu}\right)^{2}+\partial_{\mu} \bar{C} \partial^{\mu} C
$$

e a função de partição é definida por

$$
Z=\int\left(d A^{\mu}\right)(d \bar{\psi})(d \psi)(d \bar{C})(d C) \exp \left(\int_{0}^{\beta} d^{d} x \mathcal{L}_{E}\right)
$$

\footnotetext{
${ }^{1}$ Usaremos a sigla em inglês.

${ }^{2}$ As quantidades físicas devem ser independentes de calibre.
} 
Onde,

$$
\int_{0}^{\beta} d^{d} x=\int_{0}^{\beta} d \tau \int d^{d-1} x,
$$

e $\mathcal{L}_{E}$ é a lagrangiana no formalismo do tempo imaginário $x_{0}=-i \tau$. A integração funcional (4.2) se da sobre modos periódicos dos campos $A^{\mu}$ e $C$, e sobre modos anti-periódicos do campo $\psi$.

Definimos as regras de Feynman para QED na figura 4.1 seguindo as convenções de fatores combinatoriais definidas em [tHV74]. Iremos considerar apenas férmions de massa nula e iremos desprezar o potencial químico, pois as contribuições das massas dos férmions e do potencial químico são subdominantes no limite de altas temperaturas, por análise dimensional.

Veremos explicitamente na Seção 4.2 que, conforme mostrado em [Ber74], diferentemente do que ocorre a temperatura zero, a temperatura finita mesmo em teorias de calibre abelianas fantasma de Faddeev-Popov $C$ são de importância fundamental na correta contagem de graus de liberdade em um calibre covariante. Em qualquer calibre tipo Lorentz, tal como definido na seção 2.2.5, como calibre de Feynman, por exemplo, a lagrangiana de Maxwell com termo de fixação de calibre reproduz $d$ cópias da lagrangiana escalar, evidenciando a presença de graus de liberdade espúrios que devem ser subtraídos pela presença de campos fantasmas (em um espaço-tempo $d$-dimensional o fóton possui $d-2$ graus de liberdade). Neste calibre a temperatura zero, a integração funcional sobre os campos fantasmas se fatora contribuindo como uma constante multiplicativa no funcional gerador que pode ser desprezada. A temperatura finita a situação é mais complicada. Apesar do campo fantasma não se acoplar com os campos físicos, temos que os diagramas de vácuo para o campo fantasma serão dependentes da temperatura e contribuirão para pressão em ordem mais baixa, conforme veremos na próxima seção.

Iremos adotar representação de dimensão mínima para as matrizes de Dirac em que elas são de ordem $2^{E(d / 2)} \times 2^{E(d / 2)}$, onde $E(d / 2)$, que esta definida na equação 3.137$)$, é o maior inteiro menor que $d / 2$.

\subsection{Pressão ordem de um laço}

A parte livre da função de partição se fatora em contribuições independentes dos campos de calibre, campos fantasma e campos de Dirac. Os diagramas que contribuem para a função de partição estão representados na figura 4.2. A respectiva representação 

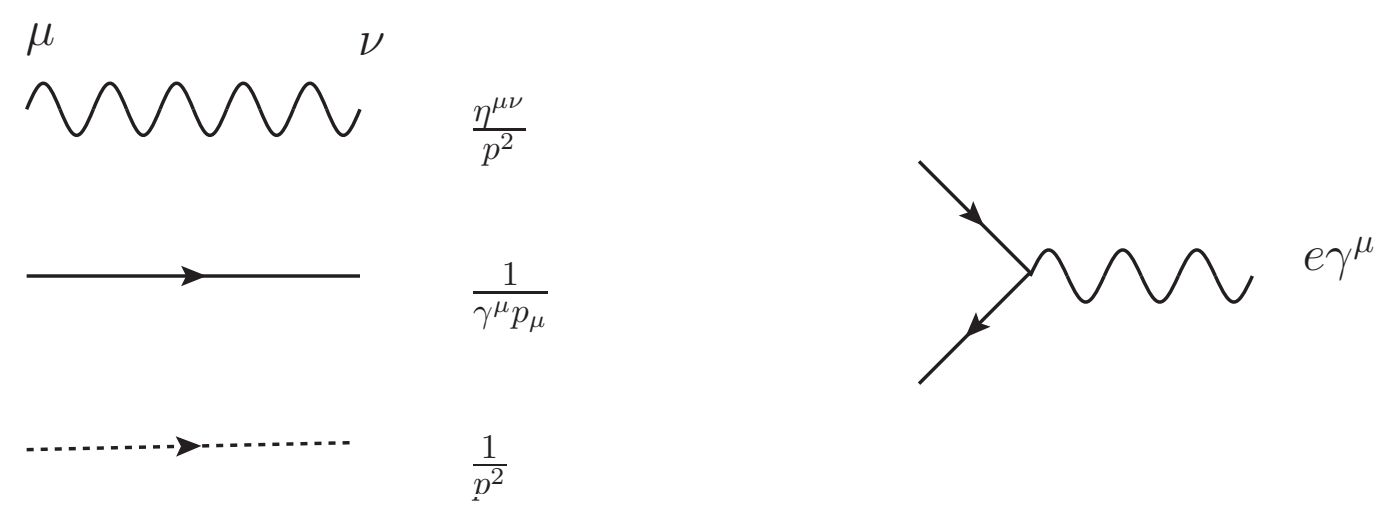

Figura 4.1: Regras de Feynman
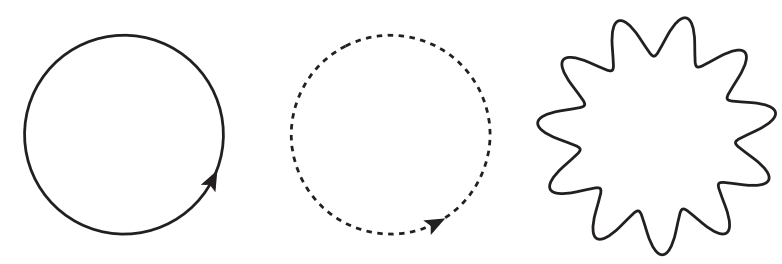

Figura 4.2: Pressão ordem de um laço

analítica é dada por [veja as equações (2.149) e (3.14]

$$
\begin{aligned}
\log Z_{0} & =V \sum_{n} \int \frac{d^{d-1} p}{(2 \pi)^{d-1}} \operatorname{tr} \log \left\{\beta \gamma_{\mu} p^{\mu}\right\}+V \sum_{n} \int \frac{d^{d-1} p}{(2 \pi)^{d-1}} \log \left\{\beta^{2}\left[\left(\omega_{n}^{B}\right)^{2}+\vec{p}^{2}\right]\right\} \\
& +d\left(-\frac{1}{2}\right) V \sum_{n} \int \frac{d^{d-1} p}{(2 \pi)^{d-1}} \log \left\{\beta^{2}\left[\left(\omega_{n}^{B}\right)^{2}+\vec{p}^{2}\right]\right\}
\end{aligned}
$$

onde $p^{0}=i \omega_{n}^{F}=\frac{i(2 n+1) \pi}{\beta}$ e $\omega_{n}^{B}=\frac{2 n \pi}{\beta}$ são as frequências de Matsubara para férmions e bósons, respectivamente.

A contribuição bosônica para a função de partição pode ser obtida a partir da função de partição do campo escalar livre (3.28) como sendo

$$
\begin{aligned}
\log Z_{0}^{B} & =(d-2)\left[-\frac{1}{2} V \sum_{n} \int \frac{d^{d-1} p}{(2 \pi)^{d-1}} \log \left\{\beta^{2}\left[\left(\omega_{n}^{B}\right)^{2}+\vec{p}^{2}\right]\right\}\right] \\
& =(d-2) \frac{V T^{d-1} \zeta(d) \Gamma(d)}{2^{d-1} \pi^{(d-1) / 2} \Gamma\left(\frac{d+1}{2}\right)} .
\end{aligned}
$$

Vale a pena ressaltar que a contribuição dos fantasmas somadas com a contribuição dos fótons resultam em $d-2$ vezes o resultado obtido no caso do campo escalar neutro, mostrando explicitamente os $d-2$ graus de liberdade dos fótons. 
O termo vindo dos férmions

$$
V \sum_{n} \int \frac{d^{d-1} p}{(2 \pi)^{d-1}} \operatorname{tr} \log \beta \gamma^{\mu} p_{\mu}=\frac{V}{2} \operatorname{tr}(I) \sum_{n} \int \frac{d^{d-1} p}{(2 \pi)^{d-1}} \log \left\{[(2 n+1) \pi]^{2}+\beta^{2}|\vec{p}|^{2}\right\}
$$

pode ser avaliado de modo semelhante. A soma de Matsubara

$$
S_{f}=\sum_{n} \log \left\{[(2 n+1) \pi]^{2}+\beta^{2}|\vec{p}|^{2}\right\}
$$

também é divergente. Vamos então extrair apenas a dependência na temperatura usando o mesmo truque

$$
\frac{\partial S_{f}}{\partial\left(\beta^{2}|\vec{p}|^{2}\right)}=\frac{-1}{\beta^{2}} \sum_{n} \frac{1}{p_{0}^{2}-|\vec{p}|^{2}}
$$

e usando o teorema dos resíduos para transformar a soma em uma integral

$$
\frac{\partial S_{f}}{\partial\left(\beta^{2}|\vec{p}|^{2}\right)}=\frac{-1}{\beta} \int_{-i \infty+\epsilon}^{i \infty+\epsilon} \frac{d p_{0}}{2 \pi i} \frac{\tanh \left(\beta p_{0} / 2\right)}{p_{0}^{2}-\vec{p}^{2}} .
$$

Resolvendo a integral de resíduo teremos

$$
\frac{\partial S_{f}}{\partial(\beta|\vec{p}|)}=\tanh (\beta|\vec{p}| / 2)
$$

e portanto

$$
\frac{\partial S_{f}}{\partial(\beta|\vec{p}|)}=2 \log [\cosh (\beta|\vec{p}| / 2)]+d_{1}=\beta|\vec{p}|+2 \log \left(1+e^{-\beta|\vec{p}|}\right)+d_{2}
$$

onde, mais uma vez, podemos desprezar as constantes divergentes. Substituindo em 4.6, teremos

$$
V \sum_{n} \int \frac{d^{d-1} p}{(2 \pi)^{d-1}} \operatorname{tr} \log \beta \gamma^{\mu} p_{\mu}=V \operatorname{tr}(I) \int \frac{d^{d-1} p}{(2 \pi)^{d-1}}\left[\frac{\beta|\vec{p}|}{2}+\log \left(1+e^{-\beta \mid \vec{p}}\right)\right],
$$

onde as constantes aditivas independentes da temperatura foram desprezadas. O primeiro termo entre colchetes é o termo advindo do estado de vácuo do oscilador harmônico, que é divergente. Desprezando este termo a integral resultante pode ser resolvida em termos 
das funções gama de Euler e zeta de Riemann,

$$
\begin{aligned}
\int d^{d-1} p \log \left(1+e^{-\beta|\vec{p}|}\right) & =\frac{2 \pi^{(d-1) / 2} T^{d-1}}{\Gamma\left(\frac{d-1}{2}\right)} \int_{0}^{\infty} d x x^{d-2} \log \left(1+e^{-x}\right) \\
& =\frac{2 \pi^{(d-1) / 2} T^{d-1}}{\Gamma\left(\frac{d-1}{2}\right)} \int_{0}^{\infty} d x \frac{x^{d-1}}{d-1} \frac{e^{-x}}{1+e^{-x}} \\
& =\frac{\pi^{(d-1) / 2} T^{d-1}}{\Gamma\left(\frac{d+1}{2}\right)} \sum_{n=1}^{\infty} \frac{(-1)^{n+1}}{n^{d}} \int_{0}^{\infty} d x e^{-x} x^{d-1} \\
& =\frac{\pi^{(d-1) / 2} T^{d-1}}{\Gamma\left(\frac{d+1}{2}\right)}\left(1-2^{1-d}\right) \zeta(d) \Gamma(d),
\end{aligned}
$$

portanto, substituindo em 4.6 teremos

$$
V \sum_{n} \int \frac{d^{d-1} p}{(2 \pi)^{d-1}} \operatorname{tr} \log \beta \gamma^{\mu} p_{\mu}=\operatorname{tr}(I) \frac{1-2^{1-d}}{(2 \sqrt{\pi})^{d-1}} \frac{\Gamma(d) \zeta(d)}{\Gamma\left(\frac{d+1}{2}\right)} V T^{d-1} .
$$

Lembrando que estamos adotando a dimensionalidade mínima para as matrizes de Dirac, temos que $\operatorname{tr}(I)=2^{E(d / 2)}$, onde $E(d / 2)$ está definido na equação 3.137 .

Juntando os resultados dos férmions 4.14) e bósons (3.28), e substituindo em 4.4

$$
\log Z_{0}=T^{d-1} V \frac{\Gamma(d) \zeta(d)}{(2 \sqrt{\pi})^{d-1} \Gamma\left(\frac{d+1}{2}\right)}\left[(d-2)+2^{E(d / 2)}\left(1-2^{(1-d)}\right)\right],
$$

e a pressão $\log Z_{0}=\beta P_{0} V$ é escrita como

$$
P_{0}=T^{d} \frac{\Gamma(d) \zeta(d)}{(2 \sqrt{\pi})^{d-1} \Gamma\left(\frac{d+1}{2}\right)}\left[(d-2)+2^{E(d / 2)}\left(1-2^{(1-d)}\right)\right] .
$$

Enfatizando, o termo proporcional a $d-2$ vem da contribuição dos campos de calibre somada à contribuição dos fantasmas de Faddeev-Popov. Este termo reproduz $d-2$ vezes a pressão do campo escalar livre, mostrando os $d-2$ graus de liberdade dos fótons em $d$ dimensões do espaço tempo. Assim, a contribuição do campo fantasma é de fundamental importância na correta contagem de graus de liberdade em 4.4), conforme mostrado em [Ber74, de modo que em $d=4$ obtemos a pressão proporcional a $T^{4}$

$$
P_{0}=T^{4} \frac{11 \pi^{2}}{180}
$$

\subsection{Pressão ordem de dois laços}

O diagrama que contribui para a pressão em ordem de dois laços está mostrado na figura 4.3 


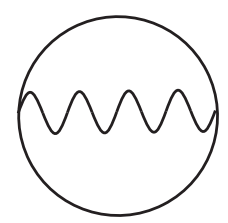

Figura 4.3: Pressão ordem de dois laços

A expressão analítica correspondente a este diagrama no calibre de Feynman é dada por

$$
\frac{\log Z_{2}}{\beta V}=-\frac{e^{2}}{2} T^{2} \sum_{n_{p} n_{q}} \int \frac{d^{d-1} p}{(2 \pi)^{d-1}} \frac{d^{d-1} q}{(2 \pi)^{d-1}} \operatorname{Tr}\left[\gamma^{\mu} \frac{1}{\not p} \gamma_{\mu} \frac{1}{\not d}\right] \frac{1}{(p-q)^{2}}
$$

Usando a identidade $1 / \not p=\gamma^{\mu} p_{\mu} / p^{2}$, reescrevemos

$$
\frac{\log Z_{2}}{\beta V}=-\frac{e^{2}}{2} T^{2} \sum_{n_{p} n_{q}} \int \frac{d^{d-1} p}{(2 \pi)^{d-1}} \frac{d^{d-1} q}{(2 \pi)^{d-1}} \operatorname{Tr}\left[\gamma^{\mu} \gamma^{\alpha} \gamma_{\mu} \gamma^{\beta}\right] \frac{p_{\alpha} q_{\beta}}{p^{2} q^{2}(p-q)^{2}}
$$

onde as somas de Matsubara se dão sobre modos ímpares, pois $p$ e $q$ são os momentos referentes as linha de férmions. Com a álgebra de Clifford e a ciclicidade do traço, podemos generalizar o cálculo do traço de quatro matrizes de Dirac para $d$ dimensões como segue:

$$
\begin{aligned}
\operatorname{Tr}\left(\gamma^{\mu} \gamma^{\alpha} \gamma^{\nu} \gamma^{\beta}\right) & =\operatorname{Tr}\left(\gamma^{\mu} \gamma^{\alpha}\left\{\gamma^{\nu}, \gamma^{\beta}\right\}\right)-\operatorname{Tr}\left(\gamma^{\mu} \gamma^{\alpha} \gamma^{\beta} \gamma^{\nu}\right) \\
& =\operatorname{Tr}\left(\gamma^{\mu} \gamma^{\alpha}\left\{\gamma^{\nu}, \gamma^{\beta}\right\}\right)-\operatorname{Tr}\left(\gamma^{\mu}\left\{\gamma^{\alpha}, \gamma^{\beta}\right\} \gamma^{\nu}\right)+\operatorname{Tr}\left(\gamma^{\mu} \gamma^{\beta} \gamma^{\alpha} \gamma^{\nu}\right) \\
& =\operatorname{Tr}\left(\gamma^{\mu} \gamma^{\alpha}\left\{\gamma^{\nu}, \gamma^{\beta}\right\}\right)-\operatorname{Tr}\left(\gamma^{\mu}\left\{\gamma^{\alpha}, \gamma^{\beta}\right\} \gamma^{\nu}\right)+\operatorname{Tr}\left(\left\{\gamma^{\mu}, \gamma^{\beta}\right\} \gamma^{\alpha} \gamma^{\nu}\right)-\operatorname{Tr}\left(\gamma^{\mu} \gamma^{\alpha} \gamma^{\nu} \gamma^{\beta}\right) \\
& =\frac{1}{4}\left[\operatorname{Tr}\left(\left\{\gamma^{\mu}, \gamma^{\alpha}\right\}\left\{\gamma^{\nu}, \gamma^{\beta}\right\}\right)-\operatorname{Tr}\left(\left\{\gamma^{\mu}, \gamma^{\nu}\right\}\left\{\gamma^{\alpha}, \gamma^{\beta}\right\}\right)+\operatorname{Tr}\left(\left\{\gamma^{\mu}, \gamma^{\beta}\right\}\left\{\gamma^{\alpha}, \gamma^{\nu}\right\}\right)\right] \\
& =2^{E(d / 2)}\left[\eta^{\mu \alpha} \eta^{\nu \beta}-\eta^{\mu \nu} \eta^{\alpha \beta}+\eta^{\mu \beta} \eta^{\nu \alpha}\right]
\end{aligned}
$$

Contraindo os índices $\mu$ e $\nu$,

$$
\operatorname{Tr}\left(\gamma^{\mu} \gamma^{\alpha} \gamma_{\mu} \gamma^{\beta}\right)=2^{E(d / 2)}(2-d) \eta^{\alpha \beta}
$$

e substituindo em 4.19) obtemos

$$
\frac{\log Z_{2}}{\beta V}=-\frac{e^{2}}{2} 2^{E(d / 2)}(2-d) T^{2} \sum_{n_{p} n_{q}} \int \frac{d^{d-1} p}{(2 \pi)^{d-1}} \frac{d^{d-1} q}{(2 \pi)^{d-1}} \frac{p \cdot q}{q^{2} p^{2}(p-q)^{2}} .
$$


Utilizando a identidade algébrica $p \cdot q=-1 / 2\left[(p-q)^{2}-q^{2}-p^{2}\right]$, reescrevemos

$$
\frac{\log Z_{2}}{\beta V}=\frac{e^{2}}{4} 2^{E(d / 2)}(2-d) T^{2} \sum_{n_{p} n_{q}} \int \frac{d^{d-1} p}{(2 \pi)^{d-1}} \frac{d^{d-1} q}{(2 \pi)^{d-1}}\left[\frac{1}{q^{2} p^{2}}-\frac{1}{p^{2}(p-q)^{2}}-\frac{1}{q^{2}(p-q)^{2}}\right] .
$$

Agora, temos que resolver duas integrais. A primeira é dada por

$$
\begin{aligned}
& T^{2} \sum_{n_{p} n_{q}} \int \frac{d^{d-1} p}{(2 \pi)^{d-1}} \frac{d^{d-1} q}{(2 \pi)^{d-1}} \frac{1}{q^{2}(p-q)^{2}} \\
& =T^{2} \sum_{n_{p} n_{q}} \int \frac{d^{d-1} p}{(2 \pi)^{d-1}} \frac{d^{d-1} q}{(2 \pi)^{d-1}} \frac{1}{\left.\left[\left(\omega_{q}^{F}\right)^{2}+\vec{q}^{2}\right)\right]\left[\left(\omega_{p}^{F}-\omega_{q}^{F}\right)^{2}+(\vec{p}-\vec{q})^{2}\right]}
\end{aligned}
$$

que pode ser fatorada em duas integrais sobre variáveis independentes fazendo uma mudança de variáveis na integral em $p$ da forma $p \rightarrow p+q$, obteremos

$$
T \sum_{n_{q}} \int \frac{d^{d-1} q}{(2 \pi)^{d-1}} \frac{1}{\left(\omega_{q}^{F}\right)^{2}+\vec{q}^{2}} T \sum_{n_{p}} \int \frac{d^{d-1} p}{(2 \pi)^{d-1}} \frac{1}{\left(\omega_{p}^{B}\right)^{2}+\vec{p}^{2}}
$$

Note que em (4.25) a soma de Matsubara em $p$ se dá sobre frequências de Matsubara bosônicas, pois a soma de duas frequências de Matsubara fermiônicas é uma frequência bosônica (a soma de dois números ímpares é um número par). A segunda integral que vamos resolver é

$$
T^{2} \sum_{n_{p} n_{q}} \int \frac{d^{d-1} p}{(2 \pi)^{d-1}} \frac{d^{d-1} q}{(2 \pi)^{d-1}} \frac{1}{q^{2} p^{2}}=\left\{T \sum_{n_{q}} \int \frac{d^{d-1} q}{(2 \pi)^{d-1}} \frac{1}{\left(\omega_{q}^{F}\right)^{2}+\vec{q}^{2}}\right\}^{2}
$$

Podemos as transformar somas de Matsubara em integrais no plano complexo usando o teorema dos resíduos, tal como mostrado no apêndice C, obtendo

$$
T \sum_{n_{q}} \int \frac{d^{d-1} q}{(2 \pi)^{d-1}} \frac{1}{-\left(i \omega_{q}^{F / B}\right)^{2}+\vec{q}^{2}}=\int_{-i \infty+\epsilon}^{i \infty+\epsilon} \frac{d q_{0}}{2 \pi i} \int \frac{d^{d-1} q}{(2 \pi)^{d-1}} \frac{1}{-\left(q_{0}\right)^{2}+\vec{q}^{2}}\left[1 \mp 2 N_{F / B}\left(q_{0}\right)\right],
$$

onde $N_{F / B}$ representa as distribuições de Fermi-Dirac e Bose-Einstein, dadas no apêndice C. O primeiro termo entre chaves é subdominante no limite de altas temperaturas e será desprezado, já o segundo termo pode ser avaliado usando o teorema dos resíduos

$$
\begin{aligned}
T \sum_{n_{q}} \int \frac{d^{d-1} q}{(2 \pi)^{d-1}} \frac{1}{-\left(i \omega_{q}^{F / B}\right)^{2}+\vec{q}^{2}} & =\mp \int \frac{d^{d-1} q}{(2 \pi)^{d-1}} \frac{N_{F / B}(|\vec{q}|)}{|\vec{q}|} \\
& =\mp \frac{2 \pi^{(d-1) / 2}}{\Gamma\left(\frac{d-1}{2}\right)(2 \pi)^{d-1}} \int_{0}^{\infty} d q q^{d-3} \frac{e^{-\beta q}}{1 \pm e^{-\beta q}}
\end{aligned}
$$




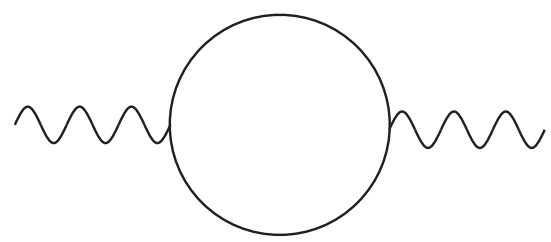

Figura 4.4: Auto-energia do fóton

A integral resultante pode ser escrita em temos das funções $\zeta$ de Riemann e $\Gamma$ de Euler, obtendo

$$
T \sum_{n_{q}} \int \frac{d^{d-1} q}{(2 \pi)^{d-1}} \frac{1}{-\left(i \omega_{q}^{F}\right)^{2}+\vec{q}^{2}}=\beta^{2-d}\left[\frac{\left(2^{4-d}-2\right) \zeta(d-2) \Gamma(d-2)}{\Gamma\left(\frac{d-1}{2}\right)(2 \sqrt{\pi})^{d-1}}\right],
$$

para o caso fermiônico e

$$
T \sum_{n_{q}} \int \frac{d^{d-1} q}{(2 \pi)^{d-1}} \frac{1}{-\left(i \omega_{q}^{F}\right)^{2}+\vec{q}^{2}}=\beta^{2-d}\left[\frac{2 \zeta(d-2) \Gamma(d-2)}{\Gamma\left(\frac{d-1}{2}\right)(2 \sqrt{\pi})^{d-1}}\right],
$$

para o caso bosônico. Substituindo 4.28 e 4.29 em 4.23), obtemos a pressão ordem de dois laços

$$
P_{2}=\frac{\log Z_{2}}{\beta V}=-e^{2} T^{2 d-4}\left\{2^{E(d / 2)}(d-2)\left(2^{3-d}-1\right)\left(2^{3-d}-3\right)\left[\frac{\Gamma(d-2) \zeta(d-2)}{\Gamma\left(\frac{d-1}{2}\right)(2 \sqrt{\pi})^{d-1}}\right]^{2}\right\} .
$$

e em $d=4$, obtemos o resultado conhecido proporcional a $T^{4}$ [Bel96, KG06,

$$
P_{2}=-e^{2} T^{4} \frac{5}{288} \quad(d=4)
$$

\subsection{Auto-energia dos fótons}

A auto-energia do fóton está representada na Figura 4.4 e possui a forma

$$
\Pi^{\mu \nu}(k)=e^{2} T \sum_{\omega_{n}^{F}} \int \frac{d^{d-1} Q}{(2 \pi)^{d-1}} \operatorname{Tr}\left(\gamma^{\mu} \frac{1}{\Phi} \gamma^{\nu} \frac{1}{\not-\not k}\right),
$$

onde $Q_{0}=i \omega_{n}^{F}=(2 n+1) \pi i T$, é a frequência de Matsubara de um férmion e $k_{0}=i \omega_{n}^{B}=$ $2 n \pi i T$ é a frequência de Matsubara de um bóson. Computando o traço temos

$$
\Pi^{\mu \nu}(k)=e^{2} 2^{E(d / 2)} T \sum_{\omega_{n}^{F}} \int \frac{d^{d-1} Q}{(2 \pi)^{d-1}} \frac{Q^{\mu}(Q-k)^{\nu}+Q^{\nu}(Q-k)^{\mu}-\eta^{\mu \nu} Q^{\alpha}(Q-k)_{\alpha}}{Q^{2}(Q-k)^{2}} .
$$


Por contagem de potências, os termos no numerador que são proporcionais ao momento externo serão subdominantes no limite de altas temperaturas. Logo

$$
\Pi^{\mu \nu}(k) \simeq e^{2} 2^{E(d / 2)} T \sum_{\omega_{n}^{F}} \int \frac{d^{d-1} Q}{(2 \pi)^{d-1}}\left[\frac{2 Q^{\mu} Q^{\nu}}{Q^{2}(Q-k)^{2}}-\frac{\eta^{\mu \nu}}{(Q-k)^{2}}\right] .
$$

Vamos agora avaliar as somas de Matsubara.

\subsubsection{As somas de Matsubara}

As somas de Matsubara que vamos avaliar possuem a forma

$$
S^{\mu \nu}=T \sum_{\omega_{n}^{F}} \frac{Q^{\mu} Q^{\nu}}{\left[Q_{0}^{2}-\vec{Q}^{2}\right]\left[\left(Q_{0}-k_{0}\right)^{2}-\vec{P}^{2}\right]}
$$

com a notação $\vec{P}=\vec{Q}-\vec{k}$. Usando o teorema dos resíduos, podemos escrever as somas de Matsubara como integrais no plano complexo

$$
S^{\mu \nu}=\int_{-i \infty+\epsilon}^{i \infty+\epsilon} \frac{d Q_{0}}{2 \pi i} \frac{1-2 N_{F}\left(Q_{0}\right)}{2}\left[\frac{Q^{\mu} Q^{\nu}}{\left[Q_{0}^{2}-\vec{Q}^{2}\right]\left[\left(Q_{0}-k_{0}\right)^{2}-\vec{P}^{2}\right]}+\left(Q_{0} \rightarrow-Q_{0}\right)\right] .
$$

Neste ponto é conveniente trabalhar componente a componente. Para as componentes espaciais teremos

$$
\begin{aligned}
S^{i j} & =\int_{-i \infty+\epsilon}^{i \infty+\epsilon} \frac{d Q_{0}}{2 \pi i} \frac{1-2 N_{F}\left(Q_{0}\right)}{2} Q^{i} Q^{j}\left[\frac{1}{\left[Q_{0}^{2}-\vec{Q}^{2}\right]\left[\left(Q_{0}-k_{0}\right)^{2}-\vec{P}^{2}\right]}+\left(Q_{0} \rightarrow-Q_{0}\right)\right] \\
& =\int_{-i \infty+\epsilon}^{i \infty+\epsilon} \frac{d Q_{0}}{2 \pi i} \frac{1-2 N_{F}\left(Q_{0}\right)}{2} Q^{i} Q^{j}\left[\frac{1}{\left[Q_{0}^{2}-\vec{Q}^{2}\right]\left[\left(Q_{0}-k_{0}\right)^{2}-\vec{P}^{2}\right]}+\left(k_{0} \rightarrow-k_{0}\right)\right] .
\end{aligned}
$$

Fechando o contorno de integração no semiplano $\operatorname{Re}\left(Q_{0}\right)>0$, podemos usar o teorema dos resíduos para obter

$$
\begin{aligned}
S^{i j} & =Q^{i} Q^{j}\left\{\frac{1-2 N_{F}(|\vec{Q}|)}{4|\vec{Q}|}\left(\frac{1}{-\left(|\vec{Q}|-k_{0}\right)^{2}+|\vec{P}|^{2}}+\frac{1}{-\left(|\vec{Q}|+k_{0}\right)^{2}+|\vec{P}|^{2}}\right)\right. \\
& \left.+\frac{1-2 N_{F}(|\vec{P}|)}{4|\vec{P}|}\left(\frac{1}{-\left(|\vec{P}|-k_{0}\right)^{2}+|\vec{Q}|^{2}}+\frac{1}{-\left(|\vec{P}|+k_{0}\right)^{2}+|\vec{Q}|^{2}}\right)\right\},
\end{aligned}
$$

onde usamos o fato que $k_{0}$ é a frequência de Matsubara de um bóson e a periodicidade 
da função de distribuição

$$
N_{F}\left(X \pm k_{0}\right)=\frac{1}{\exp (\beta[X+2 n \pi i T])+1}=N_{F}(X) .
$$

Usando a identidade algébrica

$$
\frac{1}{A^{2}-B^{2}}=\frac{1}{2 A}\left[\frac{1}{A+B}+\frac{1}{A-B}\right]
$$

podemos reescrever 4.38 como

$$
\begin{aligned}
S^{i j} & =Q^{i} Q^{j}\left[\frac{1-2 N_{F}(|\vec{Q}|)}{8|\vec{Q}||\vec{P}|}\left(\frac{1}{|\vec{P}|+|\vec{Q}|+k_{0}}+\frac{1}{|\vec{P}|-|\vec{Q}|+k_{0}}+\frac{1}{|\vec{P}|+|\vec{Q}|-k_{0}}+\frac{1}{|\vec{P}|-|\vec{Q}|-k_{0}}\right)\right. \\
& \left.+\frac{1-2 N_{F}(|\vec{P}|)}{8|\vec{Q}||\vec{P}|}\left(\frac{1}{|\vec{Q}|+|\vec{P}|+k_{0}}+\frac{1}{|\vec{Q}|-|\vec{P}|+k_{0}}+\frac{1}{|\vec{Q}|+|\vec{P}|-k_{0}}+\frac{1}{|\vec{Q}|-|\vec{P}|-k_{0}}\right)\right] .
\end{aligned}
$$

Reagrupando os termos de maneira conveniente

$$
\begin{aligned}
S^{i j} & =\frac{Q^{i} Q^{j}}{4|\vec{Q}||\vec{P}|}\left\{\left[1-N_{F}(|\vec{Q}|)-N_{F}(|\vec{P}|)\right]\left(\frac{1}{k_{0}+|\vec{Q}|+|\vec{P}|}-\frac{1}{k_{0}-|\vec{Q}|-|\vec{P}|}\right)\right. \\
& \left.+\left[N_{F}(|\vec{Q}|)-N_{F}(|\vec{P}|)\right]\left(\frac{1}{k_{0}+|\vec{Q}|-|\vec{P}|}-\frac{1}{k_{0}-|\vec{Q}|+|\vec{P}|}\right)\right\} .
\end{aligned}
$$

Como veremos, o limite de altas temperaturas de uma expressão como 4.42 pode ser obtido de maneira simples.

Para as componentes mistas de 4.36 teremos

$$
\begin{aligned}
S^{i 0} & =\int_{-i \infty+\epsilon}^{i \infty+\epsilon} \frac{d Q_{0}}{2 \pi i} \frac{1-2 N_{F}\left(Q_{0}\right)}{2} Q^{i} Q_{0}\left[\frac{1}{\left[Q_{0}^{2}-\vec{Q}^{2}\right]\left[\left(Q_{0}-k_{0}\right)^{2}-\vec{P}^{2}\right]}-\left(Q_{0} \rightarrow-Q_{0}\right)\right] \\
& =\int_{-i \infty+\epsilon}^{i \infty+\epsilon} \frac{d Q_{0}}{2 \pi i} \frac{1-2 N_{F}\left(Q_{0}\right)}{2} Q^{i}\left[\frac{Q_{0}}{\left[Q_{0}^{2}-\vec{Q}^{2}\right]\left[\left(Q_{0}-k_{0}\right)^{2}-\vec{P}^{2}\right]}-\left(k_{0} \rightarrow-k_{0}\right)\right] .
\end{aligned}
$$

Calculando as integrais de resíduo obtemos

$$
\begin{aligned}
S^{i 0} & =Q^{i}\left\{\frac{1-2 N_{F}(|\vec{Q}|)}{4|\vec{Q}|}\left(\frac{|\vec{Q}|}{-\left(|\vec{Q}|-k_{0}\right)^{2}+|\vec{P}|^{2}}-\frac{|\vec{Q}|}{-\left(|\vec{Q}|+k_{0}\right)^{2}+|\vec{P}|^{2}}\right)\right. \\
& \left.+\frac{1-2 N_{F}(|\vec{P}|)}{4|\vec{P}|}\left(\frac{|\vec{P}|+k_{0}}{-\left(|\vec{P}|+k_{0}\right)^{2}+|\vec{Q}|^{2}}-\frac{|\vec{P}|-k_{0}}{-\left(|\vec{P}|-k_{0}\right)^{2}+|\vec{Q}|^{2}}\right)\right\} .
\end{aligned}
$$

Usando as identidades algébricas

$$
\frac{1}{A^{2}-B^{2}}=\frac{1}{2 B}\left[\frac{1}{A-B}-\frac{1}{A+B}\right]=\frac{1}{2 A}\left[\frac{1}{A+B}+\frac{1}{A-B}\right],
$$


obtemos

$$
\begin{aligned}
S^{i 0} & =\frac{Q^{i}}{4|\vec{P}|}\left\{\left[1-N_{F}(|\vec{Q}|)-N_{F}(|\vec{P}|)\right]\left(\frac{-1}{k_{0}+|\vec{Q}|+|\vec{P}|}+\frac{-1}{k_{0}-|\vec{Q}|-|\vec{P}|}\right)\right. \\
& \left.-\left[N_{F}(|\vec{Q}|)-N_{F}(|\vec{P}|)\right]\left(\frac{1}{k_{0}-|\vec{Q}|+|\vec{P}|}+\frac{1}{k_{0}+|\vec{Q}|-|\vec{P}|}\right)\right\} .
\end{aligned}
$$

A componente puramente temporal de 4.36 pode ser obtida em termos das componentes espaciais em virtude da identidade algébrica

$$
Q^{2}=Q_{0}^{2}-\vec{Q}^{2}
$$

que relaciona $S^{00}$ com $S^{i j}$

\subsubsection{O limite de altas temperaturas}

Na expressão da auto-energia do fóton 4.34, a parte dependente da temperatura do termo proporcional a $\eta^{\mu \nu}$ pode ser obtida exatamente como

$$
\begin{aligned}
\int \frac{d^{d-1} Q}{(2 \pi)^{d-1}} T \sum_{\omega_{n}^{F}} \frac{1}{(Q-k)^{2}} & =\int \frac{d^{d-1} Q}{(2 \pi)^{d-1}} \int_{-i \infty+\epsilon}^{i \infty+\epsilon} \frac{d Q_{0}}{2 \pi i}\left[1-2 N_{F}\left(Q_{0}\right)\right] \frac{1}{Q_{0}^{2}-|\vec{Q}|^{2}} \\
& =\int \frac{d^{d-1} Q}{(2 \pi)^{d-1}} \frac{N_{F}(Q)}{Q}+\ldots
\end{aligned}
$$

onde $\cdots$ representa termos independentes da temperatura. A integral resultante pode ser facilmente resolvida em termos das funções zeta de Riemann e gama de Euler.

Já o outro termo

$$
\begin{aligned}
J^{\mu \nu} & =\int \frac{d^{d-1} Q}{(2 \pi)^{d-1}} T \sum_{\omega_{n}^{F}} \frac{Q^{\mu} Q^{\nu}}{Q^{2}(Q-k)^{2}} \\
& =\int \frac{d^{d-1} Q}{(2 \pi)^{d-1}} S^{\mu \nu}
\end{aligned}
$$

será avaliado no limite de altas temperaturas. Este termo é definido em termos da soma de Matsubara (4.36) $S^{\mu \nu}$ que calculamos na seção anterior. Vamos então obter o limite de altas temperaturas de $S^{\mu \nu}$.

O limite de altas temperaturas é caracterizado pela região dos "Hard Thermal Loops" que consiste na região de integração em que a escala do tri-momento do laço é muito maior que a escala do quadri-momento externo $Q \gg k$ [Bel96, FT90, BP90b], de tal maneira 
que

$$
|\vec{P}|=|\vec{Q}|+\epsilon
$$

com

$$
\epsilon=|\vec{P}|-|\vec{Q}| \simeq-\vec{k} \cdot \hat{Q}
$$

pequeno em relação a escala de temperatura. Assim teremos

$$
|\vec{P}|=\sqrt{(\vec{Q}-\vec{k})^{2}} \simeq|\vec{Q}|-\vec{k} \cdot \hat{Q}
$$

e

$$
N_{F}(|\vec{P}|) \simeq N_{F}(|\vec{Q}|)-\frac{d N_{F}(|\vec{Q}|)}{d|\vec{Q}|} \vec{k} \cdot \hat{Q}
$$

Para as componentes espacias de 4.36

$$
\begin{aligned}
S^{i j} & =\frac{Q^{i} Q^{j}}{4|\vec{Q}||\vec{P}|}\left\{-\left[N_{F}(|\vec{Q}|)+N_{F}(|\vec{P}|)\right]\left(\frac{1}{k_{0}+|\vec{Q}|+|\vec{P}|}-\frac{1}{k_{0}-|\vec{Q}|-|\vec{P}|}\right)\right. \\
& \left.+\left[N_{F}(|\vec{Q}|)-N_{F}(|\vec{P}|)\right]\left(\frac{1}{k_{0}+|\vec{Q}|-|\vec{P}|}-\frac{1}{k_{0}-|\vec{Q}|+|\vec{P}|}\right)\right\},
\end{aligned}
$$

na região de altas temperaturas teremos

$$
S^{i j} \simeq \frac{\hat{Q}^{i} \hat{Q}^{j}}{4}\left[-\frac{2 N_{F}(|\vec{Q}|)}{|\vec{Q}|}+\frac{d N_{F}(|\vec{Q}|)}{d|\vec{Q}|}\left(\frac{\vec{k} \cdot \hat{Q}}{k_{0}+\vec{k} \cdot \hat{Q}}-\frac{\vec{k} \cdot \hat{Q}}{k_{0}-\vec{k} \cdot \hat{Q}}\right)\right]
$$

Fazendo uma mudança de variáveis na integração radial do primeiro termo entre parêntesis temos

$$
\begin{aligned}
S^{i j} & \simeq \frac{\hat{Q}^{i} \hat{Q}^{j}}{4}\left[-\frac{2 N_{F}(|\vec{Q}|)}{|\vec{Q}|}-2 \frac{d N_{F}(|\vec{Q}|)}{d|\vec{Q}|} \frac{\vec{k} \cdot \hat{Q}}{k_{0}-\vec{k} \cdot \hat{Q}}\right] \\
& =\frac{\hat{Q}^{i} \hat{Q}^{j}}{4}\left[-\frac{2 N_{F}(|\vec{Q}|)}{|\vec{Q}|}+2 \frac{d N_{F}(|\vec{Q}|)}{d|\vec{Q}|}\left(1-\frac{k_{0}}{k_{0}-\vec{k} \cdot \hat{Q}}\right)\right],
\end{aligned}
$$


e substituindo em 4.48 vemos que

$$
\begin{aligned}
J^{i j} & \simeq \int \frac{d^{d-1} Q}{(2 \pi)^{d-1}} \frac{\hat{Q}^{i} \hat{Q}^{j}}{4}\left[\frac{-2 N_{F}(|\vec{Q}|)}{|\vec{Q}|}+2 \frac{d}{d|\vec{Q}|} N_{F}(|\vec{Q}|)\left(1-\frac{k_{0}}{k_{0}-\vec{k} \cdot \hat{Q}}\right)\right] \\
& =\int \frac{d^{d-1} Q}{(2 \pi)^{d-1}} \frac{\hat{Q}^{i} \hat{Q}^{j}}{2}\left[\frac{d}{d|\vec{Q}|} N_{F}(|\vec{Q}|)-\frac{N_{F}(|\vec{Q}|)}{|\vec{Q}|}\right] \\
& -\int \frac{d^{d-1} Q}{(2 \pi)^{d-1}} \frac{\hat{Q}^{i} \hat{Q}^{j}}{2} \frac{d}{d|\vec{Q}|} N_{F}(|\vec{Q}|) \frac{k_{0}}{k_{0}-\vec{k} \cdot \hat{Q}} .
\end{aligned}
$$

Para o primiro termo teremos

$$
\begin{aligned}
I^{i j} & =\int \frac{d^{d-1} Q}{(2 \pi)^{d-1}} \frac{\hat{Q}^{i} \hat{Q}^{j}}{2}\left[\frac{d}{d|\vec{Q}|} N_{F}(|\vec{Q}|)-\frac{N_{F}(|\vec{Q}|)}{|\vec{Q}|}\right] \\
& =\int \frac{d^{d-1} Q}{(2 \pi)^{d-1}} \frac{\hat{Q}^{i} \hat{Q}^{j}}{2}(1-d) \frac{N_{F}(|\vec{Q}|)}{|\vec{Q}|} \\
& =-\frac{\delta^{i j}}{2} \int \frac{d^{d-1} Q}{(2 \pi)^{d-1}} \frac{N_{F}(|\vec{Q}|)}{|\vec{Q}|},
\end{aligned}
$$

onde fizemos uma integração por partes na integração radial.

Portanto, substituindo 4.57), 4.56) e 4.47) em 4.34), obtemos

$$
\Pi^{i j} \simeq-e^{2} 2^{E(d / 2)} \int \frac{d^{d-1} Q}{(2 \pi)^{d-1}} \hat{Q}^{i} \hat{Q}^{j} \frac{d}{d|\vec{Q}|} N_{F}(|\vec{Q}|) \frac{k_{0}}{k_{0}-\vec{k} \cdot \hat{Q}},
$$

e então, fazendo uma integração por partes na integral radial, teremos

$$
\Pi^{i j} \simeq e^{2} 2^{E(d / 2)}(d-2) \int \frac{d^{d-1} Q}{(2 \pi)^{d-1}} \hat{Q}^{i} \hat{Q}^{j} \frac{N_{F}(|\vec{Q}|)}{|\vec{Q}|} \frac{k_{0}}{k_{0}-\vec{k} \cdot \hat{Q}}
$$

obtemos as componentes espaciais da auto-energia.

As componentes mistas da auto-energia são, usando (4.34),

$$
\begin{aligned}
\Pi^{i 0} & \simeq e^{2} 2^{E(d / 2)} \int \frac{d^{d-1} Q}{(2 \pi)^{d-1}} T \sum_{\omega_{n}^{F}} \frac{2 Q^{i} Q^{0}}{Q^{2}(Q-k)^{2}} \\
& =e^{2} 2^{E(d / 2)+1} \int \frac{d^{d-1} Q}{(2 \pi)^{d-1}} S^{i 0}
\end{aligned}
$$

onde a soma de Matsubara está expressa em 4.45. No limite de altas temperaturas o termo dominante de 4.45 possui a forma

$$
S^{i 0} \simeq \frac{\hat{Q}^{i}}{2} \frac{d}{d|\vec{Q}|} N_{F}(|\vec{Q}|)\left(1-\frac{k_{0}}{k_{0}-\vec{k} \cdot \hat{Q}}\right)
$$


O primeiro termo entre parêntesis é ímpar por $\vec{Q} \rightarrow-\vec{Q}$ e então se anula na integração angular. Substituindo em 4.60), temos

$$
\Pi^{i 0} \simeq-e^{2} 2^{E(d / 2)} \int \frac{d^{d-1} Q}{(2 \pi)^{d-1}} \hat{Q}^{i} \frac{d}{d|\vec{Q}|} N_{F}(|\vec{Q}|) \frac{k_{0}}{k_{0}-\vec{k} \cdot \hat{Q}},
$$

e fazendo uma integração por partes obtemos as componentes mistas da auto-energia como

$$
\Pi^{i 0} \simeq e^{2} 2^{E(d / 2)}(d-2) \int \frac{d^{d-1} Q}{(2 \pi)^{d-1}} \hat{Q}^{i} \frac{N_{F}(|\vec{Q}|)}{|\vec{Q}|} \frac{k_{0}}{k_{0}-\vec{k} \cdot \hat{Q}}
$$

As componentes puramente temporais de 4.48), são,

$$
J^{00}=\int \frac{d^{d-1} Q}{(2 \pi)^{d-1}} T \sum_{\omega_{n}^{F}} \frac{\left(Q^{0}\right)^{2}}{Q^{2}(Q-k)^{2}}
$$

e podem ser obtidas em termos das componentes espaciais usando a relação algébrica $\left(Q^{0}\right)^{2}=Q^{2}+\vec{Q}^{2}$, resultando em

$$
J^{00}=\int \frac{d^{d-1} Q}{(2 \pi)^{d-1}} T \sum_{\omega_{n}^{F}} \frac{1}{(Q-k)^{2}}+\delta_{i j} J^{i j} .
$$

Assim, substituindo 4.47, 4.56 e 4.57) em 4.65), teremos

$$
J^{00} \simeq-\frac{d-3}{2} \int \frac{d^{d-1} Q}{(2 \pi)^{d-1}} \frac{N_{F}(|\vec{Q}|)}{|\vec{Q}|}-\frac{1}{2} \int \frac{d^{d-1} Q}{(2 \pi)^{d-1}} \frac{d}{d|\vec{Q}|} N_{F}(|\vec{Q}|) \frac{k_{0}}{k_{0}-\vec{k} \cdot \hat{Q}}
$$

e portanto

$$
\Pi^{00} \simeq e^{2} 2^{E(d / 2)}(d-2) \int \frac{d^{d-1} Q}{(2 \pi)^{d-1}} \frac{N_{F}(|\vec{Q}|)}{|\vec{Q}|}\left[\frac{k_{0}}{k_{0}-\vec{k} \cdot \hat{Q}}-1\right]
$$

As componentes da auto-energia 4.59, 4.63) e 4.67) podem agora ser resumidas em uma única expressão

$$
\Pi^{\mu \nu}(k) \simeq e^{2} 2^{E(d / 2)}(d-2) \int \frac{d^{d-1} Q}{(2 \pi)^{d-1}} \frac{N_{F}(|\vec{Q}|)}{|\vec{Q}|}\left[\hat{Q}^{\mu} \hat{Q}^{\nu} \frac{k_{0}}{\hat{Q}^{\alpha} k_{\alpha}}-\delta_{0}^{\mu} \delta_{0}^{\nu}\right]
$$

com a notação $\hat{Q}^{\mu}=(1, \vec{Q} /|\vec{Q}|)$. A integração radial se fatora e pode ser resolvida de maneira simples, de modo que teremos

$$
\Pi^{\mu \nu}(k) \simeq 2 m^{2} \int \frac{d \Omega_{d-1}}{\Omega_{d-1}}\left[\hat{Q}^{\mu} \hat{Q}^{\nu} \frac{k_{0}}{\hat{Q}^{\alpha} k_{\alpha}}-\delta_{0}^{\mu} \delta_{0}^{\nu}\right]
$$


onde

$$
m^{2}=e^{2} T^{d-2} \frac{\Gamma(d-1)\left(1-2^{3-d}\right) \zeta(d-2)}{2^{d-E(d / 2)-1} \Gamma((d-1) / 2) \pi^{(d-1) / 2}},
$$

é a massa térmica do fóton ao quadrado e

$$
\Omega_{d-1}=\int d \Omega_{d-1}=\frac{2 \pi^{(d-1) / 2}}{\Gamma((d-1) / 2)},
$$

é o ângulo sólido em um espaço $(d-1)$-dimensional. Em $d=4$ temos

$$
\Pi^{\mu \nu}(k) \simeq 2 m^{2} \int \frac{d \Omega_{3}}{4 \pi}\left[\hat{Q}^{\mu} \hat{Q}^{\nu} \frac{k_{0}}{\hat{Q}^{\alpha} k_{\alpha}}-\delta_{0}^{\mu} \delta_{0}^{\nu}\right]
$$

e a massa térmica assume a forma

$$
m^{2}=\frac{e^{2} T^{2}}{6}
$$

\subsubsection{Base tensorial transversal}

Na presença de um banho térmico, a invariância de Lorentz é quebrada pelo vetor que caracteriza a quadri-velocidade do banho térmico, que será um vetor privilegiado. Desta maneira, teremos um referencial privilegiado em relação aos demais, que é o referencial em que o banho térmico está em repouso 3 , isto é, o referencial em que a quadri-velocidade do banho térmico possui a forma

$$
u^{\mu}=(1, \overrightarrow{0})=\delta_{0}^{\mu}
$$

Desta maneira, a auto-energia pode ser escrita em uma base que depende do momento $k$ e do quadri-vetor do banho térmico $u$ tal como

$$
\begin{aligned}
& t_{1}^{\mu \nu}=\eta^{\mu \nu} \\
& t_{2}^{\mu \nu}=k^{\mu} k^{\nu} \\
& t_{3}^{\mu \nu}=u^{\mu} k^{\nu}+k^{\mu} u^{\nu} \\
& t_{4}^{\mu \nu}=u^{\mu} u^{\nu} .
\end{aligned}
$$

A expressão 4.69 é transversal. Para ver isso, basta contrair 4.69 com $k$ obtendo

$$
\hat{Q}^{\nu} k_{0}-k_{0} \delta_{0}^{\mu}
$$

\footnotetext{
${ }^{3}$ Está implícito que usamos este referencial privilegiado sempre que escrevemos a soma de Masubara sobre a energia $Q_{0}=Q \cdot u$.
} 
de modo que a componente temporal deste vetor é nula e a componente espacial é ímpar por $\vec{Q} \rightarrow-\vec{Q}$ de onde vemos que

$$
k_{\mu} \Pi^{\mu \nu}(k)=0
$$

Mesmo fora da região de altas temperaturas a auto-energia do fóton é transversal. Para ver isso basta contrair a expressão 4.33 com o momento externo

$$
k_{\nu} \Pi^{\mu \nu}(k)=e^{2} 2^{E(d / 2)} T \sum_{\omega_{n}^{F}} \int \frac{d^{d-1} Q}{(2 \pi)^{d-1}}\left[\frac{Q^{\mu}\left(2 Q \cdot k-k^{2}\right)}{Q^{2}(Q-k)^{2}}-\frac{k^{\mu}}{(Q-k)^{2}}\right],
$$

e usar a identidade

$$
2 Q \cdot k-k^{2}=Q^{2}-(Q-k)^{2}
$$

de modo a obter

$$
k_{\nu} \Pi^{\mu \nu}(k)=e^{2} 2^{E(d / 2)} T \sum_{\omega_{n}^{F}} \int \frac{d^{d-1} Q}{(2 \pi)^{d-1}} \frac{Q^{\mu}-k^{\mu}}{(Q-k)^{2}} .
$$

No formalismo do tempo imaginário $k_{0}$ é uma frequência de Matsubara de um bóson, de modo que a mudança de variáveis $p \rightarrow p+k$ apenas reordena os termos na soma de Matsubara. Logo, fazendo esta mudança de variáveis obtemos que 4.79 se anula por integração simétrica, e a auto-energia dos fótons é transversal

$$
k_{\nu} \Pi^{\mu \nu}(k)=0
$$

Deste modo, é conveniente trabalhar em uma base tensorial que se beneficie da transversalidade da auto-energia. Construímos o primeiro elemento desta base como sendo o projetor no subespaço ortogonal a $k^{\mu}$

$$
T_{1}^{\mu \nu}=\eta^{\mu \nu}-\frac{k^{\mu} k^{\nu}}{k^{2}}
$$

A projeção do vetor que caracteriza o banho térmico $u$ neste subespaço tem a forma

$$
n^{\mu}=u_{\nu} T_{1}^{\mu \nu}=u^{\mu}-\frac{k \cdot u}{k^{2}} k^{\mu}
$$


de tal maneira que $k$ e $n$ são ortogonais. Deste modo definiremos a nova base como

$$
\begin{aligned}
& T_{1}^{\mu \nu}=\eta^{\mu \nu}-\frac{k^{\mu} k^{\nu}}{k^{2}} \\
& T_{2}^{\mu \nu}=\frac{k^{\mu} k^{\nu}}{k^{2}} \\
& T_{3}^{\mu \nu}=k^{\mu} n^{\nu}+n^{\mu} k^{\nu} \\
& T_{4}^{\mu \nu}=n^{\mu} n^{\nu} .
\end{aligned}
$$

nesta base, escrevemos a auto-energia do fóton como

$$
\Pi^{\mu \nu}=\sum_{i=1}^{4} c_{i} T_{i}^{\mu \nu} .
$$

Contraindo esta equação com $T_{2}^{\mu \nu}$ obtemos $c_{2}=0$. Em seguida, contraindo com $T_{3}^{\mu \nu}$ teremos $c_{3}=0$ de modo que teremos

$$
\Pi^{\mu \nu}=c_{1} T_{1}^{\mu \nu}+c_{4} T_{4}^{\mu \nu}
$$

em virtude da transversalidade.

Neste ponto, faremos uma segunda mudança de base para escrever a auto-energia em termos de dois projetores. O primeiro projetor

$$
P_{T}^{\mu \nu}=a_{1} T_{1}^{\mu \nu}+a_{4} T_{4}^{\mu \nu}
$$

é definido de modo a ser espacialmente transversal, o que significa que este tensor será ortogonal a parte espacial do momento. Desta maneria, vamos impor que

$$
\begin{aligned}
\sum_{i=1}^{d-1} k_{i} P_{T}^{i 0} & =a_{1} \sum_{i=1}^{d-1}\left[k_{i} \eta^{i 0}-k_{i} \frac{k^{i} k^{0}}{k^{2}}\right]+a_{4} \sum_{i=1}^{d-1}\left[k_{i}\left(u^{i}-\frac{u \cdot k k^{i}}{k^{2}}\right) n^{0}\right] \\
& =a_{1} \frac{k_{0} \vec{k}^{2}}{k^{2}}+a_{4} \frac{k_{0} \vec{k}^{2}}{k^{2}}\left(1-\frac{k_{0}^{2}}{k^{2}}\right) \\
& =0
\end{aligned}
$$

obtendo

$$
a_{4}=-\frac{a_{1}}{1-k_{0}^{2} / k^{2}}=\frac{k^{2}}{\vec{k}^{2}} a_{1}
$$

Agora, para conveniência futura, vamos impor que este projetor seja idempotente. Usando 
os produtos

$$
\begin{aligned}
T_{1}^{\mu \alpha} T_{1 \alpha}{ }^{\nu} & =\left(\eta^{\mu \alpha}-\frac{k^{\mu} k^{\alpha}}{k^{2}}\right)\left(\delta_{\alpha}^{\nu}-\frac{k_{\alpha} k^{\nu}}{k^{2}}\right)=T_{1}^{\mu \nu} \\
T_{4}^{\mu \alpha} T_{1 \alpha}{ }^{\nu} & =n^{\mu} n^{\alpha}\left(\delta_{\alpha}^{\nu}-\frac{k_{\alpha} k^{\nu}}{k^{2}}\right)=T_{4}^{\mu \nu} \\
T_{4}^{\mu \alpha} T_{4 \alpha}{ }^{\nu} & =n^{\mu}\left(u^{\alpha}-\frac{k \cdot u k^{\alpha}}{k^{2}}\right)\left(u_{\alpha}-\frac{k \cdot u k_{\alpha}}{k^{2}}\right) n^{\nu} \\
& =\left(1-\frac{k_{0}^{2}}{k^{2}}\right) T_{4}^{\mu \nu} \\
& =-\frac{\vec{k}^{2}}{k^{2}} T_{4}^{\mu \nu}
\end{aligned}
$$

e a expressão 4.87) obtemos

$$
\begin{aligned}
P_{T}^{\mu \alpha} P_{T \alpha}{ }^{\nu} & =a_{1}^{2} T_{1}^{\mu \alpha} T_{1 \alpha}{ }^{\nu}+2 a_{1} a_{4} T_{1}^{\mu \alpha} T_{4 \alpha}{ }^{\nu}+a_{4}^{2} T_{4}^{\mu \alpha} T_{4 \alpha}{ }^{\nu} \\
& =a_{1}^{2} T_{1}^{\mu \nu}+2 a_{1} a_{4} T_{4}^{\mu \nu}+a_{4}^{2}\left(1-\frac{k_{0}^{2}}{k^{2}}\right) T_{4}^{\mu \nu} \\
& =a_{1}\left(a_{1} T_{1}^{\mu \nu}+a_{4} T_{4}^{\mu \nu}\right) \\
& =a_{1} P_{T} .
\end{aligned}
$$

Assim, impondo $a_{1}=1$ vemos que $P_{T}^{\mu \nu}$ terá a seguinte forma idempotente

$$
P_{T}^{\mu \nu}=T_{1}^{\mu \nu}+\frac{k^{2}}{\vec{k}^{2}} T_{4}^{\mu \nu} .
$$

O segundo projetor

$$
P_{L}^{\mu \nu}=b_{1} T_{1}^{\mu \nu}+b_{4} T_{4}^{\mu \nu}
$$

será definido de modo a ser ortogonal a $P_{T}^{\mu \nu}$, isto é

$$
\begin{aligned}
P_{L}^{\mu \alpha} P_{T \alpha}{ }^{\nu} & =b_{1} T_{1}^{\mu \alpha} T_{1 \alpha}{ }^{\nu}+\left(b_{4}+b_{1} \frac{k^{2}}{\overrightarrow{k^{2}}}\right) T_{1}^{\mu \alpha} T_{4 \alpha}{ }^{\nu}+b_{4} \frac{k^{2}}{\vec{k}^{2}} T_{4}^{\mu \alpha} T_{4 \alpha}{ }^{\nu} \\
& =b_{1}\left(T_{1}^{\mu \nu}+\frac{k^{2}}{\overrightarrow{k^{2}}} T_{4}^{\mu \nu}\right) \\
& =0
\end{aligned}
$$

que implica em $b_{1}=0$. A condição de idempotência determina o parâmetro $b_{4}$. De fato,

$$
\begin{aligned}
P_{L}^{\mu \alpha} P_{L \alpha}{ }^{\nu} & =b_{4}^{2} T_{4}^{\mu \alpha} T_{4 \alpha}{ }^{\nu}=-b_{4}^{2} \frac{\vec{k}^{2}}{k^{2}} T_{4}^{\mu \alpha} \\
& =-b_{4} \frac{\vec{k}^{2}}{k^{2}} P_{L}^{\mu \nu}
\end{aligned}
$$


de modo que

$$
b_{4}=-\frac{k^{2}}{\overrightarrow{k^{2}}}
$$

Assim, os projetores possuem a forma

$$
\begin{aligned}
& P_{T}^{\mu \nu}=T_{1}^{\mu \nu}+\frac{k^{2}}{\overrightarrow{k^{2}}} T_{4}^{\mu \nu} \\
& P_{L}^{\mu \nu}=-\frac{k^{2}}{\vec{k}^{2}} T_{4}^{\mu \nu} .
\end{aligned}
$$

Em termos de componentes eles podem se escritos como

$$
\begin{aligned}
& P_{T}^{00}=P_{T}^{\mu 0}=P_{T}^{0 \mu}=0 \\
& P_{T}^{i j}=-\delta^{i j}+\frac{k^{i} k^{j}}{\vec{k}^{2}} \\
& P_{L}^{\mu \nu}=\left(\eta^{\mu \nu}-\frac{k^{\mu} k^{\nu}}{k^{2}}\right)-P_{T}^{\mu \nu} \\
& P_{L}^{00}=-\frac{\vec{k}^{2}}{k^{2}} \\
& P_{L}^{0 i}=-\frac{k_{0} k^{i}}{k^{2}} \\
& P_{L}^{i j}=-\frac{k_{0}^{2}}{k^{2}} \frac{k^{i} k^{j}}{\vec{k}^{2}} .
\end{aligned}
$$

Resumindo, na presença do banho térmico, o projetor no subespaço transversal

$$
\eta^{\mu \nu}-\frac{k^{\mu} k^{\nu}}{k^{2}}
$$

é decomposto em termos dos projetores $P_{T}$ e $P_{L}$, definidos em 4.96), ou seja,

$$
P_{T}^{\mu \nu}+P_{L}^{\mu \nu}=\eta^{\mu \nu}-\frac{k^{\mu} k^{\nu}}{k^{2}}
$$

Os projetores são transversais

$$
k_{\mu} P_{T}^{\mu \nu}=k_{\mu} P_{L}^{\mu \nu}=0
$$

ortogonais entre si

$$
P_{T}^{\mu \nu} P_{L \nu}{ }^{\alpha}=0,
$$

e idempotentes

$$
P_{T}^{\mu \nu} P_{T \nu}^{\alpha}=P_{T}^{\mu \alpha}, P_{L}^{\mu \nu} P_{L \nu}{ }^{\alpha}=P_{L}^{\mu \alpha} .
$$


Entretanto, apenas o projetor $P_{T}$ é espacialmente transversal

$$
\sum_{i} k^{i} P_{T}^{i \mu}=0
$$

Além disso, esses projetores possuem a norma dada por

$$
P_{T \nu}^{\mu} P_{T \mu}^{\nu}=P_{T \mu}^{\mu}=d-2, P_{L \nu}^{\mu} P_{L \mu}^{\nu}=P_{L \mu}^{\mu}=1 .
$$

O modo de propagação descrito pelo tensor $P_{T}$ é chamado de espacialmente transversal, enquanto o modo descrito por $P_{L}$ é chamado de espacialmente longitudinal, embora estes dois modos sejam transversais no espaço-tempo. Estes são os dois modos de propagação do fóton na presença de um meio térmico.

Nesta base a auto-energia do fóton possui a forma

$$
\Pi^{\mu \nu}=F P_{L}^{\mu \nu}+G P_{T}^{\mu \nu}
$$

onde

$$
F=\Pi^{\mu \nu} P_{L \mu \nu}=-2 m^{2} \frac{k^{2}}{\overrightarrow{k^{2}}} \int \frac{d \Omega_{d-1}}{\Omega_{d-1}} \frac{\vec{k} \cdot \hat{Q}}{k_{0}-\vec{k} \cdot \hat{Q}}
$$

e

$$
\begin{aligned}
G & =\frac{\Pi^{\mu \nu} P_{T \mu \nu}}{d-2} \\
& =-\frac{2 m^{2}}{d-2}\left[1-\frac{k^{2}}{\vec{k}^{2}} \int \frac{d \Omega_{d-1}}{\Omega_{d-1}} \frac{\vec{k} \cdot \hat{Q}}{k_{0}-\vec{k} \cdot \hat{Q}}\right] .
\end{aligned}
$$

\subsubsection{Limites estático e de grandes comprimentos de onda}

As componentes do projetor espacialmente transversal possuem a forma

$$
\begin{aligned}
& P_{T}^{\mu 0}=0 \\
& P_{T}^{i j}=-\delta^{i j}+\hat{k}^{i} \hat{k}^{j}
\end{aligned}
$$

de modo que este projetor depende apenas da direção do vetor $\vec{k}$, e assim ele não é sensível aos limites estático e de grandes comprimentos de onda. Já as componentes do projetor 
espacialmente longitudinal

$$
\begin{aligned}
P_{L}^{00} & =-\frac{\vec{k}^{2}}{k^{2}} \\
P_{L}^{0 i} & =-\frac{k_{0} k_{i}}{k^{2}}, \\
P_{L}^{i j} & =-\frac{k_{0}^{2}}{k^{2}} \frac{k^{i} k^{j}}{\vec{k}^{2}}
\end{aligned}
$$

são sensíveis a estes limites. No limite estático este projetor assume a forma

$$
P_{L}^{\mu \nu}\left(k_{0} \rightarrow 0\right)=u^{\mu} u^{\nu}
$$

e no limite de grandes comprimentos de onda temos

$$
\begin{aligned}
P_{L}^{\mu 0}(\lambda \rightarrow \infty) & =0, \\
P_{L}^{i j}(\lambda \rightarrow \infty) & =-\hat{k}^{i} \hat{k}^{j},
\end{aligned}
$$

de modo que o limites dos projetores são todos bem definidos e assim é perfeitamente consistente analisar o limite das componentes 4.105) e 4.106) isoladamente, desde que estes sejam finitos.

Em particular, o limite estático de 4.105) e 4.106 pode ser obtido de maneira trivial, de modo que teremos

$$
\Pi^{\mu \nu}\left(k_{0} \rightarrow 0\right)=-2 m^{2} u^{\mu} u^{\nu}
$$

O limite de grandes comprimentos de onda é mais delicado. Para obtê-lo vamos expandir em série de Taylor do integrando de 4.105 e 4.106 em torno de $|\vec{k}|=0$ de modo que

$$
\int \frac{d \Omega_{d-1}}{\Omega_{d-1}} \frac{\hat{Q} \cdot \vec{k}}{k_{0}-\hat{Q} \cdot \vec{k}}=\int \frac{d \Omega_{d-1}}{\Omega_{d-1}} \frac{\hat{Q} \cdot \vec{k}}{k_{0}}\left[1+\frac{\hat{Q} \cdot \vec{k}}{k_{0}}+\cdots\right],
$$

onde o primeiro termo é impar na integração angular, e assim

$$
\int \frac{d \Omega_{d-1}}{\Omega_{d-1}} \frac{\hat{Q} \cdot \vec{k}}{k_{0}-\hat{Q} \cdot \vec{k}} \simeq \int \frac{d \Omega_{d-1}}{\Omega_{d-1}}\left(\frac{\hat{Q} \cdot \vec{k}}{k_{0}}\right)^{2} .
$$

Portanto, no limite de grandes comprimentos de onda temos

$$
F(\lambda \rightarrow \infty) \simeq-2 m^{2} \int \frac{d \Omega_{d-1}}{\Omega_{d-1}}(\hat{k} \cdot \hat{Q})^{2}
$$


e

$$
G(\lambda \rightarrow \infty) \simeq-\frac{2 m^{2}}{d-2}\left[1-\int \frac{d \Omega_{d-1}}{\Omega_{d-1}}(\hat{k} \cdot \hat{Q})^{2}\right]
$$

A integral resultante está tabelada em termos da função beta. Usando algumas propriedades do ângulo sólido em dimensões arbitrárias (veja o apêndice D) escrevemos

$$
\int \frac{d \Omega_{d-1}}{\Omega_{d-1}}(\hat{Q} \cdot \hat{k})^{2}=\frac{\Omega_{d-2}}{\Omega_{d-1}} \int_{0}^{\pi} d \theta(\sin \theta)^{d-3}(\cos \theta)^{2}
$$

onde

$$
\Omega_{D}=\frac{2 \pi^{D / 2}}{\Gamma(D / 2)}
$$

é o ângulo sólido em um espaço $D$-dimensional. Fazendo $x=\cos \theta$ temos

$$
\begin{aligned}
\int \frac{d \Omega_{d-1}}{\Omega_{d-1}}(\hat{Q} \cdot \hat{k})^{2} & =\frac{\Omega_{d-2}}{\Omega_{d-1}} \int_{-1}^{1} d x\left(1-x^{2}\right)^{(d-4) / 2} x^{2} \\
& =\frac{\Omega_{d-2}}{\Omega_{d-1}} 2 \int_{0}^{1} d x\left(1-x^{2}\right)^{(d-4) / 2} x^{2} \\
& =\frac{\Omega_{d-2}}{\Omega_{d-1}} 2 \int_{0}^{1} d t(1-t)^{(d-4) / 2} t^{1 / 2}
\end{aligned}
$$

Usando a definição da função beta

$$
\mathcal{B}(a, b)=\int_{0}^{1} d t(1-t)^{b-1} t^{a-1}=\frac{\Gamma(a) \Gamma(b)}{\Gamma(a+b)},
$$

reescrevemos

$$
\begin{aligned}
\int \frac{d \Omega_{d-1}}{\Omega_{d-1}}(\hat{Q} \cdot \hat{k})^{2} & =\frac{\pi^{(d-2) / 2} \Gamma\left(\frac{d-1}{2}\right)}{\pi^{(d-1) / 2} \Gamma\left(\frac{d-2}{2}\right)} \frac{\Gamma(3 / 2) \Gamma\left(\frac{d-2}{2}\right)}{\Gamma\left(\frac{d+1}{2}\right)} \\
& =\frac{1}{d-1}
\end{aligned}
$$

e assim temos os seguintes limites

$$
\begin{aligned}
& F(\lambda \rightarrow \infty) \simeq-\frac{2 m^{2}}{d-1} \\
& G(\lambda \rightarrow \infty) \simeq-\frac{2 m^{2}}{d-1} .
\end{aligned}
$$

Substituindo na auto-energia, temos

$$
\begin{aligned}
& \Pi^{0 \mu}(\lambda \rightarrow \infty) \simeq 0 \\
& \Pi^{i j}(\lambda \rightarrow \infty) \simeq \frac{2 m^{2}}{d-1} \delta^{i j}
\end{aligned}
$$




\subsubsection{Expressão analítica}

A expressão geral da auto-energia em termos dos projetores pode ser escrita em termos da função hipergeométrica de Gauss. Para ver isso é conveniente escrever

$$
\begin{aligned}
\Pi^{\mu \nu} & =-2 m^{2} \frac{k^{2}}{\vec{k}^{2}}\left[\int \frac{d \Omega_{d-1}}{\Omega_{d-1}} \frac{\vec{k} \cdot \hat{Q}}{k_{0}-\vec{k} \cdot \hat{Q}}\right] P_{L}^{\mu \nu}-\frac{2 m^{2}}{d-2}\left[1-\frac{k^{2}}{\vec{k}^{2}} \int \frac{d \Omega_{d-1}}{\Omega_{d-1}} \frac{\vec{k} \cdot \hat{Q}}{k_{0}-\vec{k} \cdot \hat{Q}}\right] P_{T}^{\mu \nu} \\
& =-2 m^{2} \frac{k^{2}}{\vec{k}^{2}}\left[\int \frac{d \Omega_{d-1}}{\Omega_{d-1}} \frac{1}{1-z \hat{k} \cdot \hat{Q}}-1\right] P_{L}^{\mu \nu}-\frac{2 m^{2}}{d-2}\left[1-\frac{k^{2}}{\vec{k}^{2}}\left(\int \frac{d \Omega_{d-1}}{\Omega_{d-1}} \frac{1}{1-z \hat{k} \cdot \hat{Q}}-1\right)\right] P_{T}^{\mu \nu} \\
& =-2 m^{2} \frac{k^{2}}{\vec{k}^{2}}[\mathcal{I}-1] P_{L}^{\mu \nu}-\frac{2 m^{2}}{d-2}\left[1-\frac{k^{2}}{\vec{k}^{2}}(\mathcal{I}-1)\right] P_{T}^{\mu \nu}
\end{aligned}
$$

$\operatorname{com} z=|\vec{k}| / k_{0}$ e a integral $I$ está definida no apêndice D em (D.1). Usando a expressão analítica (D.5) teremos

$$
\begin{aligned}
\Pi^{\mu \nu} & =-2 m^{2} \frac{k^{2}}{\overrightarrow{k^{2}}}\left[{ }_{2} F_{1}\left(\frac{1}{2}, 1 ; \frac{d-1}{2} ; \frac{\vec{k}^{2}}{k_{0}^{2}}\right)-1\right] P_{L}^{\mu \nu} \\
& -\frac{2 m^{2}}{d-2}\left\{1-\frac{k^{2}}{\vec{k}^{2}}\left[{ }_{2} F_{1}\left(\frac{1}{2}, 1 ; \frac{d-1}{2} ; \frac{\vec{k}^{2}}{k_{0}^{2}}\right)-1\right]\right\} P_{T}^{\mu \nu} .
\end{aligned}
$$

Obviamente, esta expressão contem tanto o limite de grandes comprimentos de onda quanto o limite estático. O limite de grandes comprimentos de onda pode ser obtido diretamente da forma 4.124 escrevendo a representação integral

$$
{ }_{2} F_{1}(a, b ; c ; \xi)=\frac{\Gamma(c)}{\Gamma(c-b) \Gamma(b)} \int_{0}^{1} d t \frac{(1-t)^{c-b-1} t^{b-1}}{(1-\xi t)^{a}} .
$$

No limite de $\xi$ pequeno usando a definição da função Beta escrevemos

$$
\begin{aligned}
{ }_{2} F_{1}(a, b ; c ; \xi) & \simeq 1+a \xi \frac{\Gamma(b+1) \Gamma(c)}{\Gamma(c+1) \Gamma(b)} \\
& =1+\xi \frac{a b}{c}
\end{aligned}
$$

de modo que teremos

$$
{ }_{2} F_{1}\left(\frac{1}{2}, 1 ; \frac{d-1}{2} ; \xi\right) \simeq 1+\xi \frac{1}{d-1}
$$

e no limite de grandes comprimentos de onda obtemos

$$
\frac{k^{2}}{\overrightarrow{k^{2}}}\left[{ }_{2} F_{1}\left(\frac{1}{2}, 1 ; \frac{d-1}{2} ; \frac{\vec{k}^{2}}{k_{0}^{2}}\right)-1\right] \rightarrow \frac{1}{d-1} .
$$

Assim usando o limite de grandes comprimentos de onda dos projetores 4.107) e 4.110 
obtemos

$$
\Pi^{0 \mu}(\lambda \rightarrow \infty)=0 \quad \Pi^{i j}(\lambda \rightarrow \infty)=\frac{2 m^{2}}{d-1} \delta^{i j}
$$

que é idêntico à 4.122 .

Já para o limite estático é mais conveniente usar a representação (D.8), de modo que a auto-energia assume a forma

$$
\begin{aligned}
\Pi^{\mu \nu} & =-2 m^{2} \frac{k^{2}}{\overrightarrow{k^{2}}}\left[\frac{k_{0}^{2}}{k^{2}}{ }_{2} F_{1}\left(\frac{1}{2}, \frac{d-3}{2}, \frac{d-1}{2},-\frac{|\vec{k}|^{2}}{k^{2}}\right)-1\right] P_{L}^{\mu \nu} \\
& -\frac{2 m^{2}}{d-2}\left\{1-\frac{k^{2}}{\vec{k}^{2}}\left[\frac{k_{0}^{2}}{\vec{k}^{2}}{ }_{2} F_{1}\left(\frac{1}{2}, \frac{d-3}{2} ; \frac{d-1}{2} ;-\frac{\vec{k}^{2}}{k^{2}}\right)-1\right]\right\} P_{T}^{\mu \nu} .
\end{aligned}
$$

Usando novamente a representação integral da função hipergeométrica de Gauss 4.125, podemos ver facilmente que $k_{0}^{2}{ }_{2} F_{1}\left(1 / 2,(d-3) / 2 ;(d-1) / 2 ;-\vec{k}^{2} / k^{2}\right) \rightarrow 0$ quando $k_{0} \rightarrow 0$, e assim, usando o limite estático do projetor 4.109 obtemos

$$
\Pi^{\mu \nu}\left(k_{0} \rightarrow 0\right)=-2 m^{2} u^{\mu} u^{\nu}
$$

\subsection{A soma das divergências infravermelhas para a pressão}

Nesta seção vamos estudar a soma das divergências infravermelhas para a pressão. Quando a dimensionalidade do espaço-tempo é par veremos que as divergências infravermelhas introduzem uma não-analiticidade na série perturbativa na forma $\left(e^{2}\right)^{(d-1) / 2}$. Entretanto quando a dimensionalidade do espaço-tempo é ímpar a não-analiticidade é apenas logarítmica BFS12].

Vamos agora obter a soma das divergências infravermelhas para pressão. Em ordem de $N+1$ laços $(N \geq 2)$, o diagrama mais divergente no infravermelho é dado pelo diagrama anelar do tipo mostrado na figura 4.5, em que primeiro se corrige o propagador por meio de $N$ inserções da auto-energia em ordem de um laço, e em seguida junta-se as linhas externas do propagador corrigido.

O fator combinatorial do diagrama anelar com $N+1$ laços é contado da seguinte forma: um fator $(N-1) ! / 2$ de se ordenar $N$ inserções da auto-energia em um circulo, um fator $2^{N}$ de se trocar as linhas externas de cada auto-energia inserida, um fator $1 / N$ ! da indistinguibilidade das auto-energias inseridas e um fator $1 / 2^{N}$ como fator de simetria da 

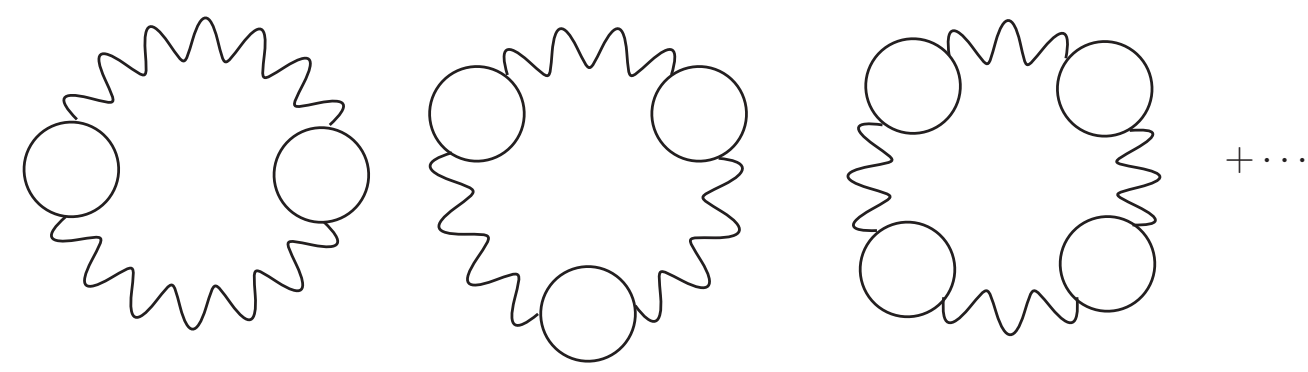

Figura 4.5: Diagramas anelares

auto-energia. Logo, o fator combinatorial é dado por

$$
\frac{1}{2 N}
$$

O integrando do diagrama anelar com $N$ inserções da auto-energia é dado por

$$
\frac{(-1)^{N}}{\left(p^{2}\right)^{N}} \Pi_{\nu_{1}}^{\mu_{1}} \Pi_{\nu_{2}}^{\nu_{1}} \Pi_{\nu_{3}}^{\nu_{2}} \ldots \Pi^{\nu_{N-2}}{ }_{\nu_{N-1}} \Pi^{\nu_{N-1}}{ }_{\mu_{1}} .
$$

Usando a expressão 4.104 para a auto-energia, e usando as propriedades 4.100, 4.101) e 4.103), reescrevemos o integrando acima como

$$
\frac{1}{\left(p^{2}\right)^{N}}\left[F^{N}+(d-2) G^{N}\right]
$$

Esta expressão mostra que, ordem a ordem, o modo estático dos diagramas anelares constituem a parte dominante no infravermelho. O diagrama anelar com $N$ inserções da auto-energia será então

$$
R_{N}=V \sum_{p_{o}} \int \frac{d^{d-1} p}{(2 \pi)^{d-1}} \frac{F^{N}+(d-2) G^{N}}{2 N\left(p^{2}\right)^{N}}
$$

A parte dominante no infravermelho deste diagrama é dada pelo modo estático, que pode ser obtido usando 4.131,

$$
R_{N}^{(e)}=V \int \frac{d^{d-1} p}{(2 \pi)^{d-1}} \frac{\left(2 m^{2}\right)^{N}}{2 N\left(-|\vec{p}|^{2}\right)^{N}}
$$

Neste ponto é mais conveniente estudar os casos de $d$ par e ímpar separadamente.

\subsubsection{Caso $d$ par}

Para $d$ par, o diagrama 4.136 é divergente no infravermelho para $N \geq d / 2$. Como estamos tomando o modo estático, a parte ultravioleta não será controlada pela distribuição de Bose-Einstein ou Fermi-Dirac como ocorre quando estamos considerando todos 
os termos da soma de Matsubara. Desta maneira, o modo estático de diagramas com $N<d / 2$ terá um comportamento ultravioleta incontrolável, embora a soma de Matsubara completa do diagrama seja finita. Portanto, considerando o caso $d$ par, a soma das divergências infravermelhas será

$$
\log Z_{\text {rings }}^{d \text { par }}=\sum_{N=d / 2}^{\infty} R_{N}^{(e)}=V \sum_{N=d / 2}^{\infty} \int \frac{d^{d-1} p}{(2 \pi)^{d-1}} \frac{\left(2 m^{2}\right)^{N}}{2 N\left(-|\vec{p}|^{2}\right)^{N}}
$$

Calculando as integrais angulares e comutando a soma com a integral radial, obtemos

$$
\log Z_{\text {rings }}^{d \text { par }}=\frac{V \Omega_{d-1}}{(2 \pi)^{d-1}} \int_{0}^{\infty} d p p^{d-2} \sum_{N=d / 2}^{\infty} \frac{(-1)^{N}\left(2 m^{2}\right)^{N}}{2 N p^{2 N}}
$$

Fazendo uma integração por partes

$\log Z_{\text {rings }}^{d \text { par }}=\frac{V \Omega_{d-1}}{(2 \pi)^{d-1} 2(d-1)}\left[\left(p^{d-1} \sum_{N=d / 2}^{\infty} \frac{\left(-2 m^{2}\right)^{N}}{N p^{2 N}}\right)_{0}^{\infty}-\int_{0}^{\infty} d p p^{d-1} \sum_{N=d / 2}^{\infty} \frac{-2\left(-2 m^{2}\right)^{N}}{p^{2 N+1}}\right]$,

podemos observar que o termo de superfície não dará contribuição pois

$$
p^{d-1} \sum_{N=d / 2}^{\infty} \frac{\left(-2 m^{2}\right)^{N}}{N p^{2 N}} \rightarrow 0(p \rightarrow \infty)
$$

$\mathrm{e}$

$$
p^{d-1} \sum_{N=d / 2}^{\infty} \frac{\left(-2 m^{2}\right)^{N}}{N p^{2 N}}=p^{d-1}\left[-\log \left(1+2 m^{2} / p^{2}\right)-\sum_{N=1}^{d / 2-1} \frac{\left(-2 m^{2}\right)^{N}}{N p^{2 N}}\right] \rightarrow 0(p \rightarrow 0)
$$

de modo que resta apenas uma integral para avaliarmos

$$
\log Z_{\text {rings }}^{d \text { par }}=\frac{V \Omega_{d-1}}{(2 \pi)^{d-1}(d-1)} \int_{0}^{\infty} d p p^{d-1} \sum_{N=d / 2}^{\infty} \frac{\left(-2 m^{2}\right)^{N}}{p^{2 N+1}}
$$

Usando a serie binomial, reescrevemos

$$
\begin{aligned}
\log Z_{\text {rings }}^{d \text { par }} & =\frac{V \Omega_{d-1}\left(-2 m^{2}\right)^{d / 2}}{(2 \pi)^{d-1}(d-1)} \int_{0}^{\infty} d p \frac{1}{p^{2}} \sum_{N=d / 2}^{\infty}\left(\frac{-2 m^{2}}{p^{2}}\right)^{N-d / 2} \\
& =\frac{V \Omega_{d-1}\left(-2 m^{2}\right)^{d / 2}}{(2 \pi)^{d-1}(d-1)} \int_{0}^{\infty} d p \frac{1}{p^{2}} \frac{1}{1+2 m^{2} / p^{2}} \\
& =\frac{(-1)^{d / 2} V \Omega_{d-1}\left(2 m^{2}\right)^{(d-1) / 2}}{(2 \pi)^{d-1}(d-1)} \int_{0}^{\infty} d x \frac{1}{1+x^{2}}
\end{aligned}
$$


A integral que restou pode ser calculada usando o teorema dos resíduos como

$$
\int_{0}^{\infty} \frac{d x}{1+x^{2}}=\frac{1}{2} \int_{-\infty}^{\infty} \frac{d x}{1+x^{2}}=\frac{1}{2} \oint_{C} \frac{d x}{1+x^{2}}=\frac{\pi}{2}
$$

onde o contorno de integração $C$ é obtido fechando o contorno no semiplano $\operatorname{Im}(x)>0$.

Com isto, obtemos uma forma fechada para a soma das divergências infravermelhas para pressão no caso $d$ par:

$$
\log Z_{\text {rings }}^{d \text { par }}=\frac{(-1)^{d / 2} V \Omega_{d-1}\left(2 m^{2}\right)^{(d-1) / 2} \pi}{2(2 \pi)^{(d-1)}(d-1)},
$$

e a pressão será

$$
P_{\text {rings }}^{d \text { par }}=\frac{(-1)^{d / 2} T \Omega_{d-1}\left(2 m^{2}\right)^{(d-1) / 2} \pi}{2(2 \pi)^{(d-1)}(d-1)},
$$

Em quatro dimensões, teremos Bel96]

$$
\log Z_{\text {rings }}^{d=4}=\frac{V}{12 \pi}\left[\frac{T^{2} e^{2}}{3}\right]^{3 / 2} \quad(d=4),
$$

e

$$
P_{\text {rings }}^{d=4}=\frac{T}{12 \pi}\left[\frac{T^{2} e^{2}}{3}\right]^{3 / 2} \quad(d=4)
$$

\subsubsection{Caso $d$ ímpar}

Quando a dimensão do espaço-tempo é ímpar, o diagrama anelar com $N$ inserções da auto-energia

$$
R_{N}^{(e)}=V \int \frac{d^{d-1} p}{(2 \pi)^{d-1}} \frac{\left(2 m^{2}\right)^{N}}{2 N\left(-|\vec{p}|^{2}\right)^{N}}
$$

será divergente no infravermelho para $N \geq \frac{d-1}{2}$. Neste caso a soma das divergências infravermelhas possui a forma

$$
\begin{aligned}
\log Z_{\text {rings }}^{\text {dímpar }} & =\sum_{N=(d-1) / 2}^{\infty} R_{N}^{(e)}=V \sum_{N=(d-1) / 2}^{\infty} \int \frac{d^{d-1} p}{(2 \pi)^{d-1}} \frac{\left(2 m^{2}\right)^{N}}{2 N\left(-|\vec{p}|^{2}\right)^{N}} \\
& =\frac{V \Omega_{d-1}\left(2 m^{2}\right)^{(d-1) / 2}}{(2 \pi)^{d-1}} \sum_{N=(d-1) / 2}^{\infty} \int_{0}^{\infty} d x x^{d-2} \frac{(-1)^{N}}{2 N x^{2 N}} .
\end{aligned}
$$

Note que no caso de $d$ par a soma começa com um divergência infravermelha linear, enquanto no cado $d$ ímpar a soma começa com uma divergência logarítmica, de modo 
que para $d$ ímpar as divergências infravermelhas são mais fracas. Note também que o primeiro termo da série 4.150 diverge logaritmicamente também no ultravioleta, de modo que precisaremos introduzir um corte na integral no $(d-1)$-momento.

A integral que teremos que tratar tem a forma

$$
I=\sum_{N=(d-1) / 2}^{\infty} \int_{0}^{\infty} d x x^{d-2} \frac{(-1)^{N}}{2 N x^{2 N}}
$$

Fazendo uma integração por partes obtemos

$$
I=\left.\sum_{N=(d-1) / 2}^{\infty} \frac{x^{d-1}}{d-1} \frac{(-1)^{N}}{2 N x^{2 N}}\right|_{0} ^{\infty}+\sum_{N=(d-1) / 2}^{\infty} \int_{0}^{\infty} d x \frac{x^{d-1}}{d-1} \frac{(-1)^{N}}{x^{2 N+1}}
$$

Neste caso o termo de superfície não se anulará, porem será finito pois

$$
\sum_{N=(d-1) / 2}^{\infty} \frac{x^{d-1}}{d-1} \frac{(-1)^{N}}{2 N x^{2 N}} \rightarrow \frac{(-1)^{(d-1) / 2}}{(d-1)^{2}}(x \rightarrow \infty)
$$

$\mathrm{e}$

$$
\sum_{N=(d-1) / 2}^{\infty} \frac{x^{d-1}}{d-1} \frac{(-1)^{N}}{2 N x^{2 N}}=\frac{x^{d-1}}{d-1}\left[\sum_{N=1}^{(d-3) / 2} \frac{(-1)^{N}}{2 N x^{2 N}}-\log \left(1+\frac{1}{x^{2}}\right)\right] \rightarrow 0(x \rightarrow 0) .
$$

Deste modo teremos

$$
\begin{aligned}
I & =\frac{(-1)^{(d-1) / 2}}{d-1}\left[\frac{1}{d-1}+\sum_{N=(d-1) / 2}^{\infty} \int_{0}^{\infty} d x \frac{1}{x} \frac{(-1)^{N-(d-1) / 2}}{x^{2[N-(d-1) / 2]}}\right] \\
& =\frac{(-1)^{(d-1) / 2}}{(d-1)}\left[\frac{1}{d-1}+\int_{0}^{\infty} \frac{d x}{x} \frac{1}{1+x^{-2}}\right]
\end{aligned}
$$

Conforme esperado, a integral resultante é logaritmicamente divergente no ultra-violeta de modo que introduziremos um corte $\mu$ na integral sobre a parte espacial do momento na primeira linha de 4.150, obtendo

$$
I=\frac{(-1)^{(d-1) / 2}}{d-1}\left[\frac{1}{d-1}+\frac{1}{2} \log \left(1+\frac{\mu^{2}}{2 m^{2}}\right)\right]
$$

e a divergência logarítmica de 4.150 se apresenta no resultado final. Com isso obtemos 


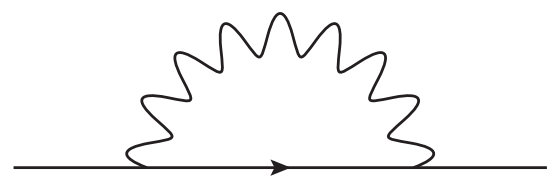

Figura 4.6: Auto-energia dos elétrons.

para a soma das divergências infravermelhas

$$
\begin{aligned}
\log Z_{\text {rings }}^{\text {dimpar }} & =\frac{V \Omega_{d-1}\left(2 m^{2}\right)^{(d-1) / 2}(-1)^{(d-1) / 2}}{2(2 \pi)^{d-1}(d-1)}\left[\frac{2}{d-1}+\log \left(1+\frac{\mu^{2}}{2 m^{2}}\right)\right] \\
& =\frac{V(-1)^{(d-1) / 2}\left(2 m^{2}\right)^{(d-1) / 2}}{2^{d} \pi^{(d-1) / 2} \Gamma\left(\frac{d+1}{2}\right)}\left[\frac{2}{d-1}+\log \left(1+\frac{\mu^{2}}{2 m^{2}}\right)\right],
\end{aligned}
$$

que contém uma não-analiticidade logarítmica na constante de acoplamento, que aparece na massa térmica do fóton. Usando a relação $\log Z=\beta P V$, obtemos o seguinte resultado para a pressão

$$
P_{\text {rings }}^{d \text { ímpar }}=\frac{T(-1)^{(d-1) / 2}\left(2 m^{2}\right)^{(d-1) / 2}}{2^{d} \pi^{(d-1) / 2} \Gamma\left(\frac{d+1}{2}\right)}\left[\frac{2}{d-1}+\log \left(1+\frac{\mu^{2}}{2 m^{2}}\right)\right] .
$$

\subsection{Auto-energia dos elétrons}

A auto-energia dos elétrons está representada na Figura 4.6. No calibre de Feynman temos a seguinte expressão analítica

$$
\begin{aligned}
\Sigma(k) & =e^{2} T \sum_{\omega_{n}^{B}} \int \frac{d^{d-1} Q}{(2 \pi)^{d-1}} \gamma^{\mu} \frac{1}{\not k-\emptyset} \gamma_{\mu} \frac{1}{Q^{2}} \\
& =e^{2} \gamma^{\mu} \gamma_{\nu} \gamma_{\mu} T \sum_{\omega_{n}^{B}} \int \frac{d^{d-1} Q}{(2 \pi)^{d-1}} \frac{(k-Q)^{\nu}}{(k-Q)^{2} Q^{2}},
\end{aligned}
$$

onde $Q_{0}=i \omega_{n}^{B}=2 n \pi i T$ é a frequência de Matsubara de um bóson e $k_{0}=i \omega_{n}^{F}=$ $(2 n+1) \pi i T$ é a frequência de Matsubara de um férmion. Estamos interessados em obter o termo dominante no limite de altas temperaturas das funções de Green. Por argumentos dimensionais, temos que os termos no numerador que são proporcionais ao momento externo serão subdominantes no limite de altas temperaturas e serão negligenciados. Logo no limite de altas temperaturas a seguinte aproximação é justificada

$$
\Sigma(k) \simeq e^{2} \gamma^{\mu} \gamma_{\nu} \gamma_{\mu} T \sum_{\omega_{n}^{B}} \int \frac{d^{d-1} Q}{(2 \pi)^{d-1}} \frac{-Q^{\nu}}{(k-Q)^{2} Q^{2}} .
$$

Feito isso vamos analisar as somas de Matsubara. 


\subsubsection{Soma de Matsubara}

As somas de Mastubara

$$
J^{\mu}=T \sum_{\omega_{n}^{B}} \frac{-Q^{\mu}}{(k-Q)^{2} Q^{2}},
$$

podem ser transformadas em um integral de contorno no plano complexo $Q_{0}$, com a ajuda do teorema dos resíduos

$$
\begin{aligned}
J^{\mu} & =\int_{-i \infty+\epsilon}^{i \infty+\epsilon} \frac{d Q_{0}}{2 \pi i}\left[\frac{1}{2}+N_{B}\left(Q_{0}\right)\right] \\
& \times\left[\frac{-Q^{\mu}}{(k-Q)^{2} Q^{2}}+\left(Q_{0} \rightarrow-Q_{0}\right)\right] .
\end{aligned}
$$

O termo independente da função de distribuição térmica, que é o termo de temperatura zero após a rotação de Wick, não será tratado aqui.

Neste ponto é conveniente trabalhar componente por componente. Para a componente temporal teremos

$$
\begin{aligned}
J_{T}^{0} & =\int_{-i \infty+\epsilon}^{i \infty+\epsilon} \frac{d Q_{0}}{2 \pi i} N_{B}\left(Q_{0}\right)\left[\frac{-Q_{0}}{(k-Q)^{2} Q^{2}}+\left(Q_{0} \rightarrow-Q_{0}\right)\right] \\
& =\int_{-i \infty+\epsilon}^{i \infty+\epsilon} \frac{d Q_{0}}{2 \pi i} N_{B}\left(Q_{0}\right)\left[\frac{-Q_{0}}{\left(Q_{0}^{2}-\vec{Q}^{2}\right)\left[\left(Q_{0}-k_{0}\right)^{2}-\vec{P}^{2}\right]}+\left(Q_{0} \rightarrow-Q_{0}\right)\right] \\
& =\int_{-i \infty+\epsilon}^{i \infty+\epsilon} \frac{d Q_{0}}{2 \pi i} N_{B}\left(Q_{0}\right)\left[\frac{-Q_{0}}{\left(Q_{0}^{2}-\vec{Q}^{2}\right)\left[\left(Q_{0}-k_{0}\right)^{2}-\vec{P}^{2}\right]}-\left(k_{0} \rightarrow-k_{0}\right)\right]
\end{aligned}
$$

com a notação $\vec{P}=\vec{Q}-\vec{k}$. Fechando o contorno de integração no semi-plano $\operatorname{Re}\left(Q_{0}\right)>0$ e usando o teorema dos resíduos avaliamos a integral como

$$
\begin{aligned}
& J_{T}^{0}=\frac{N_{B}(|\vec{Q}|)}{2\left[\left(|\vec{Q}|-k_{0}\right)^{2}-|\vec{P}|^{2}\right]}-\frac{N_{B}(|\vec{Q}|)}{2\left[\left(|\vec{Q}|+k_{0}\right)^{2}-|\vec{P}|^{2}\right]} \\
& +\frac{N_{B}\left(|\vec{P}|+k_{0}\right)\left(|\vec{P}|+k_{0}\right)}{2|\vec{P}|\left[\left(|\vec{P}|+k_{0}\right)^{2}-|\vec{Q}|^{2}\right]}-\frac{N_{B}\left(|\vec{P}|-k_{0}\right)\left(|\vec{P}|-k_{0}\right)}{2|\vec{P}|\left[\left(|\vec{P}|-k_{0}\right)^{2}-|\vec{Q}|^{2}\right]} .
\end{aligned}
$$

O fato que $k_{0}$ é a frequência de Matsubara de um férmion implica em

$$
N_{B}\left(|\vec{P}| \pm k_{0}\right)=\frac{1}{\exp (\beta[|\vec{p}| \pm i(2 n+1) \pi T])-1}=\frac{-1}{e^{\beta|\vec{p}|}+1},
$$

$\log O$

$$
N_{B}\left(|\vec{P}| \pm k_{0}\right)=-N_{F}(|\vec{P}|)
$$


de tal maneira que reescrevemos

$$
\begin{aligned}
& J_{T}^{0}=\frac{N_{B}(|\vec{Q}|)}{2\left[\left(|\vec{Q}|-k_{0}\right)^{2}-|\vec{P}|^{2}\right]}-\frac{N_{B}(|\vec{Q}|)}{2\left[\left(|\vec{Q}|+k_{0}\right)^{2}-|\vec{P}|^{2}\right]} \\
& -\frac{N_{F}(|\vec{P}|)\left(|\vec{P}|+k_{0}\right)}{2|\vec{P}|\left[\left(|\vec{P}|+k_{0}\right)^{2}-|\vec{Q}|^{2}\right]}+\frac{N_{F}(|\vec{P}|)\left(|\vec{P}|-k_{0}\right)}{2|\vec{P}|\left[\left(|\vec{P}|-k_{0}\right)^{2}-|\vec{Q}|^{2}\right]} .
\end{aligned}
$$

Lançando mão de identidades algébricas

$$
\begin{aligned}
& \frac{|\vec{P}|+k_{0}}{|\vec{P}|\left[\left(|\vec{P}|+k_{0}\right)^{2}-|\vec{Q}|^{2}\right]}=\frac{1}{2|\vec{P}|}\left[\frac{1}{|\vec{P}|+k_{0}-|\vec{Q}|}+\frac{1}{|\vec{P}|+k_{0}+|\vec{Q}|}\right], \\
& \frac{1}{\left[\left(|\vec{Q}|-k_{0}\right)^{2}-|\vec{P}|^{2}\right]}=\frac{1}{2|\vec{P}|}\left[\frac{1}{|\vec{Q}|-k_{0}-|\vec{P}|}-\frac{1}{|\vec{Q}|-k_{0}+|\vec{P}|}\right],
\end{aligned}
$$

teremos

$$
\begin{aligned}
J^{0} & =\frac{N_{B}(|\vec{Q}|)}{4|\vec{P}|}\left[\frac{1}{|\vec{Q}|-k_{0}-|\vec{P}|}-\frac{1}{|\vec{Q}|-k_{0}+|\vec{P}|}-\frac{1}{|\vec{Q}|+k_{0}-|\vec{P}|}+\frac{1}{|\vec{Q}|+k_{0}+|\vec{Q}|}\right] \\
& -\frac{N_{F}(|\vec{P}|)}{4|\vec{P}|}\left[\frac{1}{|\vec{P}|+k_{0}-|\vec{Q}|}+\frac{1}{|\vec{P}|+k_{0}+|\vec{Q}|}-\frac{1}{|\vec{P}|-k_{0}-|\vec{Q}|}-\frac{1}{|\vec{P}|-k_{0}+|\vec{Q}|}\right] .
\end{aligned}
$$

Agrupando os termos de maneira conveniente obtemos

$$
\begin{aligned}
J^{0} & =\frac{N_{B}(|\vec{Q}|)-N_{F}(|\vec{P}|)}{4|\vec{P}|}\left[\frac{1}{k_{0}+|\vec{Q}|+|\vec{P}|}+\frac{1}{k_{0}-|\vec{Q}|-|\vec{P}|}\right] \\
& -\frac{N_{F}(|\vec{Q}|)+N_{B}(|\vec{P}|)}{4|\vec{P}|}\left[\frac{1}{k_{0}+|\vec{P}|-|\vec{Q}|}+\frac{1}{k_{0}+|\vec{Q}|-|\vec{P}|}\right] .
\end{aligned}
$$

Agora nós iremos trabalhar com as componentes espaciais da soma de Matsubara

$$
J^{i}=\int_{-i \infty+\epsilon}^{i \infty+\epsilon} \frac{d Q_{0}}{2 \pi i} N_{B}\left(Q_{0}\right)\left[\frac{-Q^{i}}{\left(Q_{0}^{2}-\vec{Q}^{2}\right)\left[\left(Q_{0}-k_{0}\right)^{2}-\vec{P}^{2}\right]}+\left(Q_{0} \rightarrow-Q_{0}\right)\right] .
$$

De maneira completamente análoga ao que foi feito na componente temporal, nós podemos fechar o contorno no semiplano $\operatorname{Re}\left(Q_{0}\right)>0$, fazer as integrais de resíduo e usar 4.168 
para obter depois de alguma álgebra

$$
\begin{aligned}
J^{i} & =N_{B}(|\vec{Q}|) Q^{i}\left[\frac{1}{2|\vec{Q}|\left[\left(|\vec{Q}|-k_{0}\right)^{2}-|\vec{P}|^{2}\right]}+\frac{1}{2|\vec{Q}|\left[\left(|\vec{Q}|+k_{0}\right)^{2}-|\vec{P}|^{2}\right]}\right] \\
& -N_{F}(|\vec{P}|) Q^{i}\left[\frac{1}{2|\vec{P}|\left[\left(|\vec{P}|+k_{0}\right)^{2}-|\vec{Q}|^{2}\right]}+\frac{1}{2|\vec{P}|\left[\left(|\vec{P}|-k_{0}\right)^{2}-|\vec{Q}|^{2}\right]}\right] \\
& =\frac{N_{B}(|\vec{Q}|) Q^{i}}{4|\vec{Q}||\vec{P}|}\left[\frac{1}{|\vec{Q}|-k_{0}-|\vec{P}|}-\frac{1}{|\vec{Q}|-k_{0}+|\vec{P}|}+\frac{1}{|\vec{Q}|+k_{0}-|\vec{P}|}-\frac{1}{|\vec{Q}|+k_{0}+|\vec{P}|}\right] \\
& -\frac{N_{F}(|\vec{P}|) Q^{i}}{4|\vec{Q}||\vec{P}|}\left[\frac{1}{|\vec{P}|+k_{0}-|\vec{Q}|}-\frac{1}{|\vec{P}|+k_{0}+|\vec{Q}|}+\frac{1}{|\vec{P}|-k_{0}-|\vec{Q}|}-\frac{1}{|\vec{P}|-k_{0}+|\vec{Q}|}\right] \\
& =\frac{\left[N_{B}(|\vec{Q}|)-N_{F}(|\vec{P}|)\right] Q^{i}}{4|\vec{Q}||\vec{P}|}\left[\frac{1}{k_{0}-|\vec{Q}|-|\vec{P}|}-\frac{1}{k_{0}+|\vec{Q}|+|\vec{P}|}\right] \\
& -\frac{\left[N_{F}(|\vec{Q}|)+N_{B}(|\vec{P}|)\right] Q^{i}}{4|\vec{Q}||\vec{P}|}\left[\frac{1}{k_{0}+|\vec{P}|-|\vec{Q}|}-\frac{1}{k_{0}+|\vec{Q}|-|\vec{P}|}\right] .
\end{aligned}
$$

\subsubsection{A região dos "Hard Thermal Loops"}

Agora nós iremos obter o limite de altas temperaturas das expressões 4.170 e 4.172). Devido a presença da distribuição térmica, no limite de altas temperaturas as integrais das expressões 4.170 e 4.172 serão dominadas pela região em que o momento do laço é grande em comparação com os momentos externos que é chamada de região dos "Hard Thermal Loops" (HTL) [Bel96, FT90, BP90b]. Deste modo, na região dos HTL nos teremos $|\vec{k}| \ll|\vec{Q}|$, e assim teremos a aproximação

$$
|\vec{P}|=|\vec{Q}-\vec{k}|=|\vec{Q}|+\epsilon
$$

onde

$$
\epsilon=|\vec{P}|-|\vec{Q}|=-\hat{Q} \cdot \vec{k}+\ldots
$$

com $\epsilon$ pequeno (em comparação com a escala temperatura).

Primeiro, para a componente temporal

$$
\begin{aligned}
J^{0} & =\frac{N_{B}(|\vec{Q}|)-N_{F}(|\vec{P}|)}{4|\vec{P}|}\left[\frac{1}{k_{0}+|\vec{Q}|+|\vec{P}|}+\frac{1}{k_{0}-|\vec{Q}|-|\vec{P}|}\right] \\
& -\frac{N_{F}(|\vec{P}|)+N_{B}(|\vec{Q}|)}{4|\vec{P}|}\left[\frac{1}{k_{0}+|\vec{P}|-|\vec{Q}|}+\frac{1}{k_{0}+|\vec{Q}|-|\vec{P}|}\right],
\end{aligned}
$$


na região dos HTL vemos, por contagem de potências, que o primeiro termos é subdominante no limite de altas temperaturas, e para o segundo termo teremos

$$
J^{0} \simeq-\frac{N_{F}(|\vec{Q}|)+N_{B}(|\vec{Q}|)}{4|\vec{Q}|}\left[\frac{1}{k_{0}-\hat{Q} \cdot \vec{k}}+\frac{1}{k_{0}+\hat{Q} \cdot \vec{k}}\right]
$$

Fazendo $\vec{k} \rightarrow-\vec{k}$ na integração angular do segundo termo entre colchetes, podemos reescrever de uma maneira mais compacta,

$$
J^{0} \simeq-\frac{N_{F}(|\vec{Q}|)+N_{B}(|\vec{Q}|)}{2|\vec{Q}|} \frac{1}{k_{0}-\hat{Q} \cdot \vec{k}} .
$$

Para as componentes espaciais,

$$
\begin{aligned}
J^{i} & =\frac{N_{B}(|\vec{Q}|)-N_{F}(|\vec{P}|)}{4|\vec{P}||\vec{Q}|}\left[\frac{Q^{i}}{k_{0}-|\vec{Q}|-|\vec{P}|}-\frac{Q^{i}}{k_{0}+|\vec{P}|+|\vec{Q}|}\right] \\
& -\frac{N_{F}(|\vec{P}|)+N_{B}(|\vec{Q}|)}{4|\vec{P}||\vec{Q}|}\left[\frac{Q^{i}}{k_{0}+|\vec{P}|-|\vec{Q}|}-\frac{Q^{i}}{k_{0}+|\vec{Q}|-|\vec{P}|}\right],
\end{aligned}
$$

temos que o primeiro termo é sub-dominante, tal que

$$
J^{i} \simeq \frac{N_{F}(|\vec{Q}|)+N_{B}(|\vec{Q}|)}{4|\vec{Q}|}\left[\frac{\hat{Q}^{i}}{k_{0}+\hat{Q} \cdot \vec{k}}-\frac{\hat{Q}^{i}}{k_{0}-\hat{Q} \cdot \vec{k}}\right]
$$

Fazendo uma mudança de variáveis na integração angular do primeiro termo entre colchetes, obtemos

$$
J^{i} \simeq-\frac{N_{F}(|\vec{Q}|)+N_{B}(|\vec{Q}|)}{2|\vec{Q}|} \frac{\hat{Q}^{i}}{k_{0}-\hat{Q} \cdot \vec{k}} .
$$

Os resultados 4.177) e 4.180 podem ser escritos juntos em uma notação compacta

$$
J^{\mu}=-\frac{N_{F}(|\vec{Q}|)+N_{B}(|\vec{Q}|)}{2|\vec{Q}|} \frac{\hat{Q}^{\mu}}{k_{0}-\hat{Q} \cdot \vec{k}},
$$

onde $\hat{Q}^{\mu}=\left(1, \hat{Q}^{i}\right)$.

\subsubsection{Integração radial}

Substituindo 4.181 em 4.160 e usando a álgebra das matrizes de Dirac

$$
\begin{aligned}
\gamma^{\mu} \gamma_{\nu} \gamma_{\mu} & =\gamma^{\mu}\left\{\gamma_{\nu}, \gamma_{\mu}\right\}-\gamma^{\mu} \gamma_{\mu} \gamma_{\nu} \\
& =(2-d) \gamma_{\nu}
\end{aligned}
$$


obtemos

$$
\Sigma(k) \simeq e^{2}(2-d) \gamma_{\nu} \int \frac{d^{d-1} Q}{(2 \pi)^{d-1}} \frac{N_{F}(|\vec{Q}|)+N_{B}(|\vec{Q}|)}{2|\vec{Q}|} \frac{-\hat{Q}^{\nu}}{k_{0}-\hat{Q} \cdot \vec{k}} .
$$

A integração radial é fatorada de modo que temos

$$
\Sigma(k) \simeq \frac{e^{2} T^{d-2}(d-2) \Omega_{d-1} \gamma_{\nu}}{2(2 \pi)^{d-1}} \int_{0}^{\infty} d y y^{d-3}\left(\frac{1}{e^{y}-1}+\frac{1}{e^{y}+1}\right) \int \frac{d \Omega_{d-1}}{\Omega_{d-1}} \frac{\hat{Q}^{\nu}}{k_{0}-\hat{Q} \cdot \vec{k}} .
$$

Resolvendo as integrais como

$$
\int_{0}^{\infty} d y y^{d-3} \frac{1}{e^{y}-1}=\zeta(d-2) \Gamma(d-2)
$$

e

$$
\int_{0}^{\infty} d y y^{d-3} \frac{1}{e^{y}+1}=\left(1-2^{3-d}\right) \zeta(d-2) \Gamma(d-2)
$$

escrevemos

$$
\Sigma(k) \simeq \frac{e^{2} T^{d-2}\left(2-2^{3-d}\right) \zeta(d-2) \Gamma(d-1) \Omega_{d-1} \gamma_{\mu}}{2(2 \pi)^{d-1}} \int \frac{d \Omega_{d-1}}{\Omega_{d-1}} \frac{\hat{Q}^{\mu}}{k_{0}-\hat{Q} \cdot \vec{k}} .
$$

É conveniente definir a massa térmica do elétron como

$$
m_{F}^{2}=e^{2} T^{d-2} \frac{\left(2-2^{3-d}\right) \zeta(d-2) \Gamma(d-1)}{2^{d-1} \pi^{(d-1) / 2} \Gamma\left(\frac{d-1}{2}\right)}
$$

de tal maneira que

$$
\Sigma(k) \simeq m_{F}^{2} \int \frac{d \Omega_{d-1}}{\Omega_{d-1}} \frac{\hat{\phi}}{k_{0}-\hat{Q} \cdot \vec{k}}
$$

No caso $d=4$ teremos $[$ Bel96]

$$
m_{F}^{2}=\frac{e^{2} T^{2}}{8}, \quad(d=4)
$$

e a integração angular é trivial fornecendo o resultado conhecido Bel96]

$$
\Sigma(k) \simeq \frac{m_{F}^{2}}{|\vec{k}|}\left\{\gamma_{0} \log \left(\frac{k_{0}+|\vec{k}|}{k_{0}-|\vec{k}|}\right)+\vec{\gamma} \cdot \hat{k}\left[1-\frac{k_{0}}{2|\vec{k}|} \log \left(\frac{k_{0}+|\vec{k}|}{k_{0}-|\vec{k}|}\right)\right]\right\}, \quad(d=4)
$$




\subsubsection{Integração angular}

Como vimos, a auto-energia do elétron é expressa em termos da integral vetorial

$$
\mathcal{J}^{\mu}=\int \frac{d \Omega_{d-1}}{\Omega_{d-1}} \frac{\hat{Q}^{\mu}}{k_{0}-\hat{Q} \cdot \vec{k}} .
$$

Para a componente temporal teremos

$$
\begin{aligned}
\mathcal{J}^{0} & =\int \frac{d \Omega_{d-1}}{\Omega_{d-1}} \frac{1}{k_{0}-\hat{Q} \cdot \vec{k}} \\
& =\frac{1}{k_{0}} \mathcal{I},
\end{aligned}
$$

onde $\mathcal{I}$ é a integral escalar

$$
\mathcal{I}=\int \frac{d \Omega_{d-1}}{\Omega_{d-1}} \frac{k_{0}}{k_{0}-\hat{Q} \cdot \vec{k}} .
$$

As componentes espaciais também podem ser expressas em termos da mesma integral escalar

$$
\begin{aligned}
\mathcal{J}^{i} & =\int \frac{d \Omega_{d-1}}{\Omega_{d-1}} \frac{\hat{Q}^{i}}{k_{0}-\hat{Q} \cdot \vec{k}} \\
& =\frac{k^{i}}{|\vec{k}|^{2}}(\mathcal{I}-1) .
\end{aligned}
$$

Alternativamente, podemos expressar a integral vetorial em termos de uma base constituída pelo vetor que caracteriza o banho térmico $u^{\mu}$ e pelo momento externo

$$
\mathcal{J}^{\mu}=u^{\mu} \frac{k_{0}}{|\vec{k}|^{2}}\left(1-\frac{k^{2}}{k_{0}^{2}} \mathcal{I}\right)+\frac{k^{\mu}}{|\vec{k}|^{2}}(\mathcal{I}-1) .
$$

A integral $\mathcal{I}$ pode ser expressa em termos da função hipergeométrica de Gauss tal como feito no Apêndice $\mathrm{D}$ como

$$
\begin{aligned}
\mathcal{I} & ={ }_{2} F_{1}\left(\frac{1}{2}, 1 ; \frac{d-1}{2} ; \frac{\vec{k}^{2}}{k_{0}^{2}}\right) \\
& =\frac{k_{0}^{2}}{k^{2}}{ }_{2} F_{1}\left(\frac{1}{2}, \frac{d-3}{2}, \frac{d-1}{2},-\frac{|\vec{k}|^{2}}{k^{2}}\right) .
\end{aligned}
$$

Deste modo, substituindo 4.196 e 4.197) em 4.189 obtemos a representação analítica da auto-energia do elétron em termos da função hipergeométrica de Gauss. 


\subsubsection{Limite estático}

No formalismo do tempo imaginário, a componente temporal do momento externo do férmion é uma frequência de Matsubara

$$
k_{0}=i(2 n+1) \pi T \text {, }
$$

que é diferente de zero para todo $n$. Como a temperatura é elevada, não faz sentido considerar o limite desta energia tendendo a zero pois ela é um múltiplo discreto da temperatura. Entretanto, depois que calculamos a soma de Matsubara e usamos a relação (4.166), podemos, de maneira consistente, fazer uma continuação analítica na energia externa para valores arbitrários no plano complexo. Assim, neste ponto é consistente avaliar o limite estático da auto-energia do férmion.

Para obter o limite estático, é mais conveniente trabalhar componente por componente. Para a componente temporal temos

$$
\begin{aligned}
\mathcal{J}^{0} & =\frac{1}{k_{0}}+\frac{1}{k_{0}} \int \frac{d \Omega_{d-1}}{\Omega_{d-1}} \frac{\hat{Q} \cdot \vec{k}}{k_{0}-\hat{Q} \cdot \vec{k}} \\
& =\frac{1}{k_{0}}-\frac{1}{k_{0}} \int \frac{d \Omega_{d-1}}{\Omega_{d-1}} \frac{1}{1-k_{0} / \hat{Q} \cdot \vec{k}} .
\end{aligned}
$$

Fazendo uma expansão em série de Taylor para $k_{0}$ pequeno obtemos

$$
\mathcal{J}^{0}=-\frac{1}{k_{0}} \int \frac{d \Omega_{d-1}}{\Omega_{d-1}}\left[\frac{k_{0}}{\hat{Q} \cdot \vec{k}}+\left(\frac{k_{0}}{\hat{Q} \cdot \vec{k}}\right)^{2}+\cdots\right]
$$

vemos que o primeiro termo entre colchetes se anula na integração angular, de tal maneira que tomando o limite $k_{0} \rightarrow 0$ esta componente irá se anular. Logo,

$$
\mathcal{J}^{0}\left(k_{0} \rightarrow 0\right)=0
$$

Para as componentes espaciais

$$
\mathcal{J}^{i}=\frac{\hat{k}^{i}}{|\vec{k}|} \int \frac{d \Omega_{d-1}}{\Omega_{d-1}} \frac{\hat{Q} \cdot \vec{k}}{k_{0}-\hat{Q} \cdot \vec{k}},
$$

temos que o limite estático é completamente bem definido

$$
\mathcal{J}^{i}\left(k_{0} \rightarrow 0\right)=-\frac{\hat{k}^{i}}{|\vec{k}|}
$$


Desde modo, a auto-energia do elétron no limite estático adquire a forma simples

$$
\Sigma\left(k_{0}=0\right)=-m_{F}^{2} \frac{\vec{\gamma} \cdot \vec{k}}{\vec{k}^{2}} .
$$

Alternativamente, podemos obter o limite estático diretamente da representação analítica 4.196 usando a segunda linha de 4.197 e o fato de que a função hipergeométrica de Gauss é regular quando o seu último argumento tende a 1.

\subsubsection{Limite de grandes comprimentos de onda}

O limite de grandes comprimento de onda também pode ser obtido de maneira semelhante, tratando a integral angular componente por componente. Para as componentes espaciais

$$
\mathcal{J}^{i}=\int \frac{d \Omega_{d-1}}{\Omega_{d-1}} \frac{\hat{Q}^{i}}{k_{0}-\hat{Q} \cdot \vec{k}},
$$

vemos que o limite de grandes comprimentos de onda é bem definido e se anula pois é ímpar na integração angular

$$
\mathcal{J}^{i}(\lambda \rightarrow \infty)=0
$$

Para a componente temporal

$$
\mathcal{J}^{0}=\int \frac{d \Omega_{d-1}}{\Omega_{d-1}} \frac{1}{k_{0}-\hat{Q} \cdot \vec{k}}
$$

o limite de grandes comprimentos de onda possui a forma

$$
\mathcal{J}^{0}(\lambda \rightarrow \infty)=\frac{1}{k_{0}}
$$

de modo que

$$
\Sigma(\lambda \rightarrow \infty)=m_{F}^{2} \frac{\gamma_{0}}{k_{0}} .
$$

Obviamente, o limite de grandes comprimentos de onda também pode ser obtido da expressão analítica 4.196), só que neste caso usando a primeira linha de 4.197) e o fato que a hipergeométrica de Gauss tende a 1 quando o seu último argumento se anula. 


\subsubsection{Invariância de calibre}

Nesta seção vamos mostrar que a auto-energia dos elétrons é invariante de calibre no limite de altas temperaturas. Em um calibre covariante geral temos

$$
\begin{aligned}
\Sigma_{\xi}(k) & =e^{2} T \sum_{\omega_{n}^{B}} \int \frac{d^{d-1} Q}{(2 \pi)^{d-1}} \gamma^{\mu} \frac{1}{\not k-\varnothing} \gamma^{\nu} \frac{\eta_{\mu \nu}-(1-\xi) Q_{\mu} Q_{\nu} / k^{2}}{Q^{2}} \\
& =e^{2} T \sum_{\omega_{n}^{B}} \int \frac{d^{d-1} Q}{(2 \pi)^{d-1}} \gamma^{\mu} \gamma^{\alpha} \gamma^{\nu} \frac{(k-Q)_{\alpha}\left[\eta_{\mu \nu}-(1-\xi) Q_{\mu} Q_{\nu} / Q^{2}\right]}{(k-Q)^{2} Q^{2}}
\end{aligned}
$$

No calibre de Feynman, $\xi=1$. Para mostrar a invariância de calibre nós teremos que mostrar que o termo

$$
\Xi=T \sum_{\omega_{n}^{B}} \int \frac{d^{d-1} Q}{(2 \pi)^{d-1}} \frac{\gamma^{\mu} \gamma^{\alpha} \gamma^{\nu}(k-Q)_{\alpha} Q_{\mu} Q_{\nu}}{(k-Q)^{2}\left(Q^{2}\right)^{2}},
$$

é subdominante no limite de altas temperaturas. Para ver isso basta notar que

$$
\Xi=T \sum_{\omega_{n}^{B}} \int \frac{d^{d-1} Q}{(2 \pi)^{d-1}} \gamma^{\mu} \gamma^{\alpha} \gamma^{\nu}\left[\frac{(k-Q)_{\alpha} k_{\mu} Q_{\nu}}{(k-Q)^{2}\left(Q^{2}\right)^{2}}-\frac{(k-Q)_{\alpha}(k-Q)_{\mu} Q_{\nu}}{(k-Q)^{2}\left(Q^{2}\right)^{2}}\right],
$$

onde o primeiro termo é subdominante no limite de altas temperaturas, e o segundo termo

$$
\begin{aligned}
& -T \sum_{\omega_{n}^{B}} \int \frac{d^{d-1} Q}{(2 \pi)^{d-1}} \frac{\gamma^{\mu} \gamma^{\alpha} \gamma^{\nu}(k-Q)_{\alpha}(k-Q)_{\mu} Q_{\nu}}{(k-Q)^{2}\left(Q^{2}\right)^{2}} \\
& =-T \sum_{\omega_{n}^{B}} \int \frac{d^{d-1} Q}{(2 \pi)^{d-1}} \frac{\gamma^{\nu} Q_{\nu}}{\left(Q^{2}\right)^{2}}=0,
\end{aligned}
$$

se anula por integração simétrica. Portanto, o termo dependente de calibre da auto-energia dos elétrons é subdominante no limite de altas temperaturas.

\subsection{Função de vértice}

A função de vértice na QED, representada na figura 4.7 possui a forma

$$
e \Gamma^{\mu}\left(P_{1}, P_{2}\right)=e^{3} T \sum_{k_{0}} \int \frac{d^{d-1} k}{(2 \pi)^{d-1}} \gamma^{\alpha} \frac{1}{\not P_{1}-\not k} \gamma^{\mu} \frac{1}{P_{2}-\not k} \gamma_{\alpha} \frac{1}{k^{2}},
$$




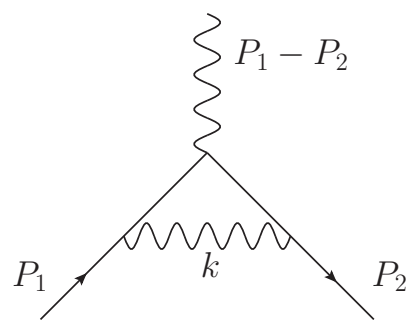

Figura 4.7: Função de vértice na QED

com $k_{0}=2 n \pi i T$ sendo a frequência de Matsubara do fóton interno. Esta expressão pode ser reescrita como

$$
\Gamma^{\mu}\left(P_{1}, P_{2}\right)=e^{2} T \sum_{k_{0}} \int \frac{d^{d-1} k}{(2 \pi)^{d-1}} \gamma^{\alpha} \frac{\not P_{1}-\not k}{\left(k-P_{1}\right)^{2}} \gamma^{\mu} \frac{\not P_{2}-\not k}{\left(k-P_{2}\right)^{2}} \gamma_{\alpha} \frac{1}{k^{2}} .
$$

Os termos no numerador que são proporcionais ao momento externo serão subdominantes no limite de altas temperaturas de modo que teremos

$$
\Gamma^{\mu}\left(P_{1}, P_{2}\right) \simeq e^{2} T \sum_{k_{0}} \int \frac{d^{d-1} k}{(2 \pi)^{d-1}} \frac{\gamma^{\alpha} \not k \gamma^{\mu} \not k \gamma_{\alpha}}{\left(k-P_{1}\right)^{2}\left(k-P_{2}\right)^{2} k^{2}} .
$$

usando a álgebra das matrizes de Dirac obtemos

$$
\Gamma^{\mu}\left(P_{1}, P_{2}\right) \simeq e^{2}(2-d) T \sum_{k_{0}} \int \frac{d^{d-1} k}{(2 \pi)^{d-1}} \frac{2 \not k k^{\mu}-\gamma^{\mu} k^{2}}{\left(k-P_{1}\right)^{2}\left(k-P_{2}\right)^{2} k^{2}} .
$$

Para o termo proporcional a $k^{2}$ temos a seguinte soma de Matsubara

$$
\Upsilon=\int \frac{d^{d-1} k}{(2 \pi)^{d-1}} T \sum_{k_{0}=i \omega_{B}} \frac{1}{\left(k-P_{1}\right)^{2}\left(k-P_{2}\right)^{2}} .
$$

Fazendo uma mudança de variáveis na integração em $k$ reescrevemos

$$
\Upsilon=\int \frac{d^{d-1} q}{(2 \pi)^{d-1}} T \sum_{q_{0}=i \omega_{F}} \frac{1}{q^{2}(q-P)^{2}},
$$

com a notação $P=P_{2}-P_{1}$. Deste modo, para a parte dependente da temperatura teremos

$$
\Upsilon_{\beta}=-\int \frac{d^{d-1} q}{(2 \pi)^{d-1}} \int_{-i \infty+\epsilon}^{i \infty+\epsilon} \frac{d q_{0}}{2 \pi i} N_{F}\left(q_{0}\right)\left(\frac{1}{\left(q_{0}^{2}-\vec{q}^{2}\right)\left[\left(q_{0}-P_{0}\right)^{2}-(\vec{q}-\vec{P})^{2}\right]}+\left(q_{0} \rightarrow-q_{0}\right)\right) .
$$

fechando o contorno de integração no semi-plano $\operatorname{Re}\left(q_{0}\right)>0$ e usando o teorema dos 
resíduos obtemos

$$
\begin{aligned}
\Upsilon_{\beta} & =\int \frac{d^{d-1} q}{(2 \pi)^{d-1}}\left[\frac{N_{F}(|\vec{q}|)}{2|\vec{q}|}\left(\frac{1}{\left(|\vec{q}|-P_{0}\right)^{2}-(\vec{q}-\vec{P})^{2}}+\frac{1}{\left(|\vec{q}|+P_{0}\right)^{2}-(\vec{q}-\vec{P})^{2}}\right)\right. \\
& \left.+\frac{N_{F}(|\vec{q}-\vec{P}|)}{2|\vec{q}-\vec{P}|}\left(\frac{1}{\left(|\vec{q}-\vec{P}|+P_{0}\right)^{2}-\vec{q}^{2}}+\frac{1}{\left(|\vec{q}-\vec{P}|-P_{0}\right)^{2}-\vec{q}^{2}}\right)\right]
\end{aligned}
$$

Usando a identidade algébrica

$$
\frac{1}{A^{2}-B^{2}}=\frac{1}{2 B}\left[\frac{1}{A-B}-\frac{1}{A+B}\right]
$$

reescrevemos

$$
\begin{aligned}
\Upsilon_{\beta} & =\int \frac{d^{d-1} q}{(2 \pi)^{d-1}}\left[\frac{N_{F}(|\vec{q}|)+N_{F}(|\vec{q}-\vec{P}|)}{4|\vec{q}||\vec{q}-\vec{P}|}\left(\frac{1}{q_{0}-|\vec{q}-\vec{P}|-\vec{P}}-\frac{1}{q_{0}+|\vec{q}-\vec{P}|+\vec{P}}\right)\right. \\
& \left.+\frac{N_{F}(|\vec{q}-\vec{P}|)-N_{F}(|\vec{q}|)}{4|\vec{q}||\vec{q}-\vec{P}|}\left(\frac{1}{q_{0}+|\vec{q}-\vec{P}|-\vec{P}}-\frac{1}{q_{0}-|\vec{q}-\vec{P}|+\vec{P}}\right)\right] .
\end{aligned}
$$

Na região de HTL teremos

$$
|\vec{q}-\vec{P}| \simeq|\vec{q}|-\vec{P} \cdot \hat{q}
$$

e

$$
N_{F}(|\vec{q}-\vec{P}|) \simeq N_{F}(|\vec{q}|)-\vec{P} \cdot \hat{q} \frac{d N_{F}(|\vec{q}|)}{d|\vec{q}|}
$$

Logo, o termo dominante no limite de altas temperaturas será

$$
\Upsilon_{\beta} \simeq \int \frac{d^{d-1} q}{(2 \pi)^{d-1}}\left[\frac{N_{F}(|\vec{q}|)}{|\vec{q}|^{3}}+\frac{N_{F}(|\vec{q}|)}{2|\vec{q}|^{2}} \frac{\vec{P} \cdot \hat{q}}{q_{0}-\vec{P} \cdot \hat{q}}\right]_{q_{0}=|\vec{q}|} .
$$

Esta integral possui um comportamento análogo a função de dois pontos para a teoria $\phi^{3}$ [BF12]. Logo, ela possui um comportamento do tipo $\log (T)$ em $d=4$ e será subdominante no limite de altas temperaturas.

Assim teremos

$$
\Gamma^{\mu}\left(P_{1}, P_{2}\right) \simeq e^{2}(2-d) \sum_{k_{0}} \int \frac{d^{d-1} k}{(2 \pi)^{d-1}} \frac{2 \not k k^{\mu}}{\left(k-P_{1}\right)^{2}\left(k-P_{2}\right)^{2} k^{2}} .
$$

Deste modo, para a função de vértice a soma de Matsubara que vamos avaliar é

$$
\Theta^{\mu \nu}=\sum_{k_{0}=i \omega_{n}^{B}} \frac{k^{\mu} k^{\nu}}{k^{2}\left(k-P_{1}\right)^{2}\left(k-P_{2}\right)^{2}} .
$$


Com a ajuda do teorema de resíduos, esta soma pode ser escrita como uma integral no plano complexo e a parte térmica será

$$
\Theta^{\mu \nu}=\int_{-i \infty+\epsilon}^{i \infty+\epsilon} \frac{d k_{0}}{2 \pi i} N_{B}\left(k_{0}\right)\left[\frac{k^{\mu} k^{\nu}}{\left(k_{0}^{2}-\vec{k}^{2}\right)\left[\left(k_{0}-P_{10}^{2}\right)-w_{1}^{2}\right]\left[\left(k_{0}-P_{20}^{2}\right)-w_{2}^{2}\right]}+\left(k_{0} \rightarrow-k_{0}\right)\right]
$$

onde $P_{s 0}$ é a componente temporal do quadri-vetor $P_{s}$ e $w_{s}=\left|\vec{k}-\vec{P}_{s}\right|$, para $s=1,2$.

Para as componentes espaciais teremos

$$
\begin{aligned}
\Theta_{\beta}^{i j} & =-k^{i} k^{j}\left\{\frac{N_{B}(|\vec{k}|)}{2|\vec{k}|}\left[\frac{1}{\left[\left(|\vec{k}|-P_{10}\right)^{2}-w_{1}^{2}\right]\left[\left(|\vec{k}|-P_{20}\right)^{2}-w_{2}^{2}\right]}+\left(P_{10}, P_{20} \rightarrow-P_{10},-P_{20}\right)\right]\right. \\
& -\frac{N_{F}\left(w_{1}\right)}{2 w_{1}}\left[\frac{1}{\left[\left(w_{1}+P_{10}\right)^{2}-|\vec{k}|^{2}\right]\left[\left(w_{1}+P_{10}-P_{20}\right)^{2}-w_{2}^{2}\right]}+\left(P_{10}, P_{20} \rightarrow-P_{10},-P_{20}\right)\right] \\
& \left.-\frac{N_{F}\left(w_{2}\right)}{2 w_{2}}\left[\frac{1}{\left[\left(w_{2}+P_{20}\right)^{2}-|\vec{k}|^{2}\right]\left[\left(w_{2}+P_{20}-P_{10}\right)^{2}-w_{1}^{2}\right]}+\left(P_{10}, P_{20} \rightarrow-P_{10},-P_{20}\right)\right]\right\} .
\end{aligned}
$$

Usando a identidade algébrica

$$
\begin{aligned}
\frac{1}{A^{2}-B^{2}} \frac{1}{C^{2}-D^{2}} & =\frac{1}{4 B D}\left[\frac{1}{A-B} \frac{1}{C-D}+\frac{1}{A+B} \frac{1}{C+D}\right. \\
& \left.-\frac{1}{A-B} \frac{1}{C+D}-\frac{1}{A+B} \frac{1}{C-D}\right]
\end{aligned}
$$

Como temos termos do tipo $N_{B}(|\vec{k}|)$ e $N_{F}\left(w_{s}\right)$ teremos que no limite de altas temperaturas

$$
N_{F}\left(w_{s}\right)=N_{F}(|\vec{k}|)
$$

e em (4.231) apenas o termo com duas diferenças no denominador serão dominantes no limite de altas temperaturas (veja o cálculo do limite de altas temperaturas da autoenergia dos férmions na seção 4.6.2 e assim

$$
\frac{1}{A^{2}-B^{2}} \frac{1}{C^{2}-D^{2}} \simeq \frac{1}{4 B D} \frac{1}{A-B} \frac{1}{C-D}
$$


e assim temos

$$
\begin{aligned}
\Theta^{i j} & =-\frac{k^{i} k^{j}}{8|\vec{k}| \omega_{1} \omega_{2}}\left\{N_{B}(|\vec{k}|)\left[\frac{1}{\left(|\vec{k}|-P_{10}-w_{1}\right)\left(|\vec{k}|-P_{20}-w_{2}\right)}+\left(P_{10}, P_{20} \rightarrow-P_{10},-P_{20}\right)\right]\right. \\
& -N_{F}\left(w_{1}\right)\left[\frac{1}{\left(w_{1}+P_{10}-|\vec{k}|\right)\left(w_{1}+P_{01}-P_{02}-w_{2}\right)}+\left(P_{10}, P_{20} \rightarrow-P_{10},-P_{20}\right)\right] \\
& \left.-N_{F}\left(w_{2}\right)\left[\frac{1}{\left(w_{2}+P_{20}-|\vec{k}|\right)\left(w_{2}+P_{02}-P_{01}-w_{1}\right)}+\left(P_{10}, P_{20} \rightarrow-P_{10},-P_{20}\right)\right]\right\},
\end{aligned}
$$

e assim no limite de altas temperaturas teremos

$$
\begin{aligned}
\Theta_{\beta}^{i j} & \simeq-\frac{\hat{k}^{i} \hat{k}^{j}}{4|\vec{k}|}\left[\frac{N_{B}(|\vec{k}|)}{\left(P_{10}-\vec{P}_{1} \cdot \hat{k}\right)\left(P_{20}-\vec{P}_{2} \cdot \hat{k}\right)}-\frac{N_{F}(|\vec{k}|)}{\left(P_{10}-\vec{P}_{1} \cdot \hat{k}\right)\left[P_{10}-P_{02}-\left(\vec{P}_{1}-\vec{P}_{2}\right) \cdot \hat{k}\right]}\right. \\
& \left.-\frac{N_{F}(|\vec{k}|)}{\left(P_{10}-\vec{P}_{1} \cdot \hat{k}\right)\left[P_{10}-P_{02}-\left(\vec{P}_{1}-\vec{P}_{2}\right) \cdot \hat{k}\right]}\right]
\end{aligned}
$$

que pode ser reescrito como

$$
\Theta_{\beta}^{i j} \simeq-\frac{\hat{k}^{i} \hat{k}^{j}}{4|\vec{k}|} \frac{N_{B}(|\vec{k}|)+N_{F}(|\vec{k}|)}{\left(P_{10}-\vec{P}_{1} \cdot \hat{k}\right)\left(P_{20}-\vec{P}_{2} \cdot \hat{k}\right)}
$$

Para as componentes mistas teremos

$$
\Theta_{\beta}^{0 i}=\int_{-i \infty+\epsilon}^{i \infty+\epsilon} \frac{d k_{0}}{2 \pi i} N_{B}\left(k_{0}\right) k^{i}\left[\frac{k_{0}}{\left(k_{0}^{2}-\vec{k}^{2}\right)\left[\left(k_{0}-P_{10}^{2}\right)-w_{1}^{2}\right]\left[\left(k_{0}-P_{20}^{2}\right)-w_{2}^{2}\right]}+\left(k_{0} \rightarrow-k_{0}\right)\right],
$$

e usando o teorema dos resíduos

$$
\begin{aligned}
\Theta_{\beta}^{0 i} & =-k^{i}\left\{\frac{N_{B}(|\vec{k}|)}{2|\vec{k}|}\left[\frac{|\vec{k}|}{\left[\left(|\vec{k}|-P_{10}\right)^{2}-w_{1}^{2}\right]\left[\left(|\vec{k}|-P_{20}\right)^{2}-w_{2}^{2}\right]}-\left(P_{10}, P_{20} \rightarrow-P_{10},-P_{20}\right)\right]\right. \\
& -\frac{N_{F}\left(w_{1}\right)}{2 w_{1}}\left[\frac{w_{1}+P_{10}}{\left[\left(w_{1}+P_{10}\right)^{2}-|\vec{k}|^{2}\right]\left[\left(w_{1}+P_{10}-P_{20}\right)^{2}-w_{2}^{2}\right]}-\left(P_{10}, P_{20} \rightarrow-P_{10},-P_{20}\right)\right] \\
& \left.-\frac{N_{F}\left(w_{2}\right)}{2 w_{2}}\left[\frac{w_{2}+P_{20}}{\left[\left(w_{2}+P_{20}\right)^{2}-|\vec{k}|^{2}\right]\left[\left(w_{2}+P_{20}-P_{10}\right)^{2}-w_{1}^{2}\right]}-\left(P_{10}, P_{20} \rightarrow-P_{10},-P_{20}\right)\right]\right\} .
\end{aligned}
$$

Neste caso, para o primeiro termo vamos usar a mesma identidade 4.231, mas para o 
segundo e o terceiro termo usaremos

$$
\begin{aligned}
\frac{1}{A^{2}-B^{2}} \frac{1}{C^{2}-D^{2}} & =\frac{1}{4 B C}\left[\frac{1}{A-B} \frac{1}{C-D}+\frac{1}{A-B} \frac{1}{C+D}\right. \\
& \left.-\frac{1}{A+B} \frac{1}{C-D}-\frac{1}{A+B} \frac{1}{C+D}\right]
\end{aligned}
$$

e aqui os mesmos argumentos valem. Portanto, apenas termo com duas diferenças serão dominantes no limite de altas temperaturas de modo que teremos

$$
\begin{aligned}
\Theta^{0 i} & =-\frac{k^{i}}{8 \omega_{1} \omega_{2}}\left\{N_{B}(|\vec{k}|)\left[\frac{1}{\left(|\vec{k}|-P_{10}-w_{1}\right)\left(|\vec{k}|-P_{20}-w_{2}\right)}-\left(P_{10}, P_{20} \rightarrow-P_{10},-P_{20}\right)\right]\right. \\
& -N_{F}\left(w_{1}\right)\left[\frac{1}{\left(w_{1}+P_{10}-|\vec{k}|\right)\left(w_{1}+P_{01}-P_{02}-w_{2}\right)}-\left(P_{10}, P_{20} \rightarrow-P_{10},-P_{20}\right)\right] \\
& \left.-N_{F}\left(w_{2}\right)\left[\frac{1}{\left(w_{2}+P_{20}-|\vec{k}|\right)\left(w_{2}+P_{02}-P_{01}-w_{1}\right)}-\left(P_{10}, P_{20} \rightarrow-P_{10},-P_{20}\right)\right]\right\} .
\end{aligned}
$$

O limite de altas temperaturas o termo dominante será

$$
\Theta_{\beta}^{0 i} \simeq-\frac{\hat{k}^{i}}{4|\vec{k}|} \frac{N_{B}(|\vec{k}|)+N_{F}(|\vec{k}|)}{\left(P_{10}-\vec{P}_{1} \cdot \hat{k}\right)\left(P_{20}-\vec{P}_{2} \cdot \hat{k}\right)}
$$

Para a componente temporal, usamos a identidade

$$
k_{0}^{2}=k^{2}+\vec{k}^{2}
$$

para escrever

$$
\Theta^{00}=\eta_{i j} \Theta^{i j}+\Upsilon
$$

e como o segundo termo é subdominante no limite de altas temperaturas, temos

$$
\Theta^{00} \simeq-\frac{1}{4|\vec{k}|} \frac{N_{B}(|\vec{k}|)+N_{F}(|\vec{k}|)}{\left(P_{10}-\vec{P}_{1} \cdot \hat{k}\right)\left(P_{20}-\vec{P}_{2} \cdot \hat{k}\right)}
$$

e assim teremos

$$
\Theta^{\mu \nu} \simeq \frac{\hat{k}^{\mu} \hat{k}^{\nu}}{4|\vec{k}|} \frac{N_{B}(|\vec{k}|)+N_{F}(|\vec{k}|)}{\left(P_{10}-\vec{P}_{1} \cdot \hat{k}\right)\left(P_{20}-\vec{P}_{2} \cdot \hat{k}\right)}
$$

com a notação $\hat{k}^{\mu}=(1, \hat{k})$. Deste modo teremos

$$
\Gamma^{\mu}\left(P_{1}, P_{2}\right) \simeq e^{2}(2-d) \int \frac{d^{d-1} k}{(2 \pi)^{d-1}} \frac{k \hat{k}^{\mu}}{2|\vec{k}|} \frac{N_{B}(|\vec{k}|)+N_{F}(|\vec{k}|)}{\left(P_{10}-\vec{P}_{1} \cdot \hat{k}\right)\left(P_{20}-\vec{P}_{2} \cdot \hat{k}\right)},
$$




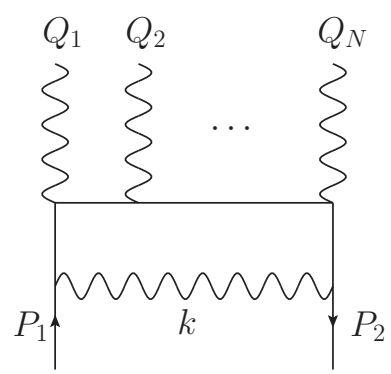

Figura 4.8: Função de vértice com $N$ bósons e 2 férmions na QED.

e a integral radial é a mesma que no caso da auto-energia do elétron (veja seção 4.6.3) de modo que teremos

$$
\Gamma^{\mu}\left(P_{1}, P_{2}\right) \simeq-m_{F}^{2} \int \frac{d \Omega_{d-1}}{\Omega_{d-1}} \frac{\hat{k} \hat{k}^{\mu}}{\left(P_{10}-\vec{P}_{1} \cdot \hat{k}\right)\left(P_{20}-\vec{P}_{2} \cdot \hat{k}\right)} .
$$

Esta expressão para função de vértice de tres pontos está relacionada com a função de dois férmions por uma identidade de Ward

$$
\left(P_{1}-P_{2}\right)_{\mu} \Gamma^{\mu}\left(P_{1}, P_{2}\right)=\Sigma\left(P_{1}\right)-\Sigma\left(P_{2}\right)
$$

que é um caso particular das identidades de Ward que deduziremos na próxima seção.

\subsection{Identidades de Ward}

A identidade 4.248) é um caso especial das identidades de Ward. Vamos deduzir uma classe das identidades de Ward que relacionam as funções de vértice de $N$ e $N-1$ bósons externos ambas com 2 férmions externos (as funções de vértice com 2 férmions externos são as únicas funções de vértices que são dominantes no limite de altas temperaturas no caso da QED [Bel96]). A função de vértice na QED com $N$ linhas bosônicas externas possui a forma

$$
\begin{aligned}
\Gamma_{\mu_{1} \cdots \mu_{N}}\left(P_{1}, P_{2}, Q_{1}, \ldots, Q_{N}\right) & =\sum_{\text {permt. }} e^{2} T \sum_{k_{0}} \int \frac{d^{d-1} p}{(2 \pi)^{d-1}} \gamma^{\alpha} \frac{1}{\not P_{1}-\not k} \gamma_{\mu_{1}} \frac{1}{\not P_{1}+Q_{1}-\not k} \cdots \\
& \times \gamma_{\mu_{N}} \frac{1}{P_{1}+\phi_{1}+\cdots+\phi_{N}-k} \gamma_{\alpha} \frac{1}{k^{2}} \\
& =\sum_{\text {permt. }} \Gamma_{\mu_{1} \cdots \mu_{N}}^{(N)}\left(P_{1}, P_{2}, Q_{1}, \ldots, Q_{N}\right)
\end{aligned}
$$

onde a soma se da sobre todas permutações das linhas externas bosônicas e $k_{0}=2 n \pi i T$ é a frequência de Matsubara de um bóson. A conservação do quadri-momento fornece o 
vínculo

$$
Q_{1}+\cdots+Q_{N}=P_{2}-P_{1}
$$

Usando o fato que os termos do numerador proporcionais aos momentos externos serão subdominantes no limite de altas temperaturas temos

$$
\begin{aligned}
\Gamma_{\mu_{1} \cdots \mu_{N}}^{(N)}\left(P_{1}, P_{2}, Q_{1}, \ldots, Q_{N}\right) & \simeq e^{2} T \sum_{k_{0}} \int \frac{d^{d-1} k}{(2 \pi)^{d-1}}(-1)^{N+1} \gamma^{\alpha} \gamma_{\lambda_{1}} \gamma_{\mu_{1}} \cdots \gamma_{\lambda_{N}} \gamma_{\mu_{N}} \gamma_{\lambda_{N+1}} \gamma_{\alpha} \\
& \frac{k^{\lambda_{1}} \cdots k^{\lambda_{N+1}}}{\left(P_{1}-k\right)^{2}\left(P_{1}+Q_{1}-k\right)^{2} \cdots\left(P_{1}+Q_{1}+\cdots+Q_{N}-k\right)^{2} k^{2}}
\end{aligned}
$$

Usando

$$
\gamma_{\lambda_{1}} \gamma_{\mu_{1}} \gamma_{\lambda_{2}} k^{\lambda_{1}} k^{\lambda_{2}}=2 \not k k_{\mu_{1}}-k^{2} \gamma_{\mu_{1}}
$$

e notando que o termo proporcional a $k^{2}$ será subdominante no limite de altas temperaturas assim como em 4.218, e assin no limite de altas temperaturas teremos

$$
\gamma_{\lambda_{1}} \gamma_{\mu_{1}} \gamma_{\lambda_{2}} k^{\lambda_{1}} k^{\lambda_{2}} \simeq 2 \not k k_{\mu_{1}}
$$

e repetindo o mesmo raciocínio obtemos

$$
\gamma_{\lambda_{1}} \gamma_{\mu_{1}} \cdots \gamma_{\lambda_{N}} \gamma_{\mu_{N}} \gamma_{\lambda_{N+1}} k^{\lambda_{1}} \cdots k^{\lambda_{N+1}} \simeq 2^{N} \not k k_{\mu_{1}} \cdots k_{\mu_{N}}
$$

e

$$
\gamma^{\alpha} \gamma_{\lambda_{1}} \gamma_{\mu_{1}} \cdots \gamma_{\lambda_{N}} \gamma_{\mu_{N}} \gamma_{\lambda_{N+1}} \gamma_{\alpha} k^{\lambda_{1}} \cdots k^{\lambda_{N+1}} \simeq(2-d) 2^{N} \not k k_{\mu_{1}} \cdots k_{\mu_{N}}
$$

de modo que

$$
\begin{aligned}
\Gamma_{\mu_{1} \cdots \mu_{N}}^{(N)}\left(P_{1}, P_{2}, Q_{1}, \ldots, Q_{N}\right) & \simeq e^{2}(2-d) 2^{N}(-1)^{N+1} T \sum_{k_{0}} \int \frac{d^{d-1} k}{(2 \pi)^{d-1}} \\
& \frac{\not k k_{\mu_{1}} \cdots k_{\mu_{N}}}{\left(P_{1}-k\right)^{2}\left(P_{1}+Q_{1}-k\right)^{2} \cdots\left(P_{1}+Q_{1}+\cdots+Q_{N}-k\right)^{2} k^{2}} .
\end{aligned}
$$


Como a expressão 4.256 é simétrica nos índices $\mu_{1} \cdots \mu_{N}$, a soma sobre a permutação sobre as linhas externas pode ser escrita como

$$
\Gamma_{\mu_{1} \cdots \mu_{N}}\left(P_{1}, P_{2}, Q_{1}, \ldots, Q_{N}\right)=\sum_{\substack{i_{1}, \cdots, i_{N}=1 \\ i_{1} \neq \cdots \neq i_{N}}}^{N} \Gamma_{\mu_{1} \cdots \mu_{N}}^{(N)}\left(P_{1}, P_{2}, Q_{i_{1}}, \ldots, Q_{i_{N}}\right)
$$

Para verificar a identidade de Ward é conveniente reescrever a soma sobre as permutações como

$$
\begin{aligned}
\Gamma_{\mu_{1} \cdots \mu_{N}}\left(P_{1}, P_{2}, Q_{1}, \ldots, Q_{N}\right) & =\sum_{\substack{i_{2}, \cdots, i_{N}=2 \\
i_{2} \neq \cdots \neq i_{N}}}^{N}\left[\Gamma_{\mu_{1} \cdots \mu_{N}}^{(N)}\left(P_{1}, P_{2}, Q_{1}, Q_{i_{2}} \ldots, Q_{i_{N}}\right)\right. \\
& \left.+\cdots+\Gamma_{\mu_{1} \cdots \mu_{N}}^{(N)}\left(P_{1}, P_{2}, Q_{i_{2}}, \ldots, Q_{i_{N}}, Q_{1}\right)\right] .
\end{aligned}
$$

Contraindo 4.256 com $Q_{1}^{\mu_{1}}$ e usando que no limite de altas temperaturas temos para o numerador

$$
Q_{1} \cdot k \simeq \frac{1}{2}\left[\left(Q_{j_{1}}+\cdots+Q_{j_{l}}-k\right)^{2}-\left(Q_{j_{1}}+\cdots+Q_{j_{l}}+Q_{1}-k\right)^{2}\right]
$$

teremos

$$
\begin{aligned}
& Q_{1}^{\mu_{1}} \Gamma_{\mu_{1} \cdots \mu_{N}}^{(N)}\left(P_{1}, P_{2}, Q_{1}, Q_{i_{2}} \ldots, Q_{i_{N}}\right)=\Gamma_{\mu_{2} \cdots \mu_{N}}^{(N-1)}\left(P_{1}, P_{2}, Q_{i_{2}}+Q_{1} \ldots, Q_{i_{N}}\right) \\
&-\Gamma_{\mu_{2} \cdots \mu_{N}}^{(N-1)}\left(P_{1}+Q_{1}, P_{2}, Q_{i_{2}} \ldots, Q_{i_{N}}\right), \\
& Q_{1}^{\mu_{1}} \Gamma_{\mu_{1} \cdots \mu_{N}}^{(N)}\left(P_{1}, P_{2}, Q_{i_{2}} \ldots, Q_{i_{n}}, Q_{1}, Q_{i_{n+1}}, \ldots, Q_{i_{N}}\right) \\
&=\Gamma_{\mu_{2} \cdots \mu_{N}}^{(N-1)}\left(P_{1}, P_{2}, Q_{i_{2}} \ldots, Q_{i_{n}}, Q_{i_{n+1}}+Q_{1} \ldots, Q_{i_{N}}\right) \\
&-\Gamma_{\mu_{2} \cdots \mu_{N}}^{(N-1)}\left(P_{1}, P_{2}, Q_{i_{2}} \ldots, Q_{i_{n}}+Q_{1}, Q_{i_{n+1}} \ldots, Q_{i_{N}}\right)
\end{aligned}
$$

e

$$
\begin{aligned}
Q_{1}^{\mu_{1}} \Gamma_{\mu_{1} \cdots \mu_{N}}^{(N)}\left(P_{1}, P_{2}, Q_{i_{2}} \ldots, Q_{i_{N}}, Q_{1}\right) & =\Gamma_{\mu_{2} \cdots \mu_{N}}^{(N-1)}\left(P_{1}, P_{2}-Q_{1}, Q_{i_{2}} \ldots, Q_{i_{N}}\right) \\
& -\Gamma_{\mu_{2} \cdots \mu_{N}}^{(N-1)}\left(P_{1}, P_{2}, Q_{i_{2}} \ldots, Q_{i_{N}}+Q_{1}\right) .
\end{aligned}
$$

Deste modo, substituindo 4.260, 4.261) e 4.262 em 4.258, obtemos

$$
\begin{aligned}
Q_{1}^{\mu_{1}} \Gamma_{\mu_{1} \cdots \mu_{N}}\left(P_{1}, P_{2}, Q_{1}, \ldots, Q_{N}\right) & =\sum_{\substack{i_{2}, \cdots, i_{N}=2 \\
i_{2} \neq \cdots \neq i_{N}}}^{N}\left[\Gamma_{\mu_{2} \cdots \mu_{N}}^{(N-1)}\left(P_{1}, P_{2}-Q_{1}, Q_{i_{2}} \ldots, Q_{i_{N}}\right)\right. \\
& \left.-\Gamma_{\mu_{2} \cdots \mu_{N}}^{(N-1)}\left(P_{1}+Q_{1}, P_{2}, Q_{i_{2}} \ldots, Q_{i_{N}}\right)\right]
\end{aligned}
$$


e reconhecendo a função de $N-1$ pontos, temos a identidade de Ward

$$
\begin{aligned}
Q_{1}^{\mu_{1}} \Gamma_{\mu_{1} \cdots \mu_{N}}\left(P_{1}, P_{2}, Q_{1}, \ldots, Q_{N}\right) & =\Gamma_{\mu_{2} \cdots \mu_{N}}\left(P_{1}, P_{2}-Q_{1}, Q_{i_{2}} \ldots, Q_{i_{N}}\right) \\
& -\Gamma_{\mu_{2} \cdots \mu_{N}}\left(P_{1}+Q_{1}, P_{2}, Q_{i_{2}} \ldots, Q_{i_{N}}\right)
\end{aligned}
$$

\subsection{Lagrangiana efetiva térmica}

No limite de altas temperaturas, apenas as funções de dois pontos dos fótons e dos elétrons e as funções de vértice de $N$-fótons e dois elétrons sobrevivem. Assim, a lagrangiana efetiva dos fótons é trivial de ser obtida em termos auto-energia dos fótons 4.69

$$
\Pi^{\mu \nu}(k) \simeq 2 m^{2} \int \frac{d \Omega_{d-1}}{\Omega_{d-1}}\left[\hat{Q}^{\mu} \hat{Q}^{\nu} \frac{k_{0}}{\hat{Q}^{\alpha} k_{\alpha}}-\delta_{0}^{\mu} \delta_{0}^{\nu}\right],
$$

e assin no espaço dos momentos teremos

$$
\begin{aligned}
\mathcal{L}_{\gamma} & =\frac{1}{2} A_{\mu}(k) \Pi^{\mu \nu}(k) A_{\nu}(k) \\
& =m^{2} \int \frac{d \Omega_{d-1}}{\Omega_{d-1}}\left[A \cdot \hat{Q} \frac{k_{0}}{\hat{Q}^{\alpha} k_{\alpha}} A \cdot \hat{Q}+A_{0}^{2}\right]
\end{aligned}
$$

e no espaço de configurações teremos

$$
\mathcal{L}_{\gamma}=m^{2} \int \frac{d \Omega_{d-1}}{\Omega_{d-1}}\left[A \cdot \hat{Q} \frac{\partial_{0}}{\hat{Q}^{\alpha} \partial_{\alpha}} A \cdot \hat{Q}+A_{0}^{2}\right] .
$$

Já para o setor dos elétrons uma lagrangiana efetiva que gera a função de dois pontos possui a forma

$$
\begin{aligned}
\mathcal{L}_{F}^{(2)} & =\bar{\psi}(k) \Sigma(k) \psi(-k) \\
& =m_{F}^{2} \bar{\psi}(k) \int \frac{d \Omega_{d-1}}{\Omega_{d-1}} \frac{\hat{\phi}}{\hat{Q}^{\alpha} k_{\alpha}} \psi(-k)
\end{aligned}
$$

e no espaço de configurações escrevemos

$$
\mathcal{L}_{F}^{(2)}=m_{F}^{2} \bar{\psi} \int \frac{d \Omega_{d-1}}{\Omega_{d-1}} \frac{i \hat{\phi}}{\hat{Q}^{\alpha} \partial_{\alpha}} \psi .
$$

As identidades de Ward entre as funções de Green envolvendo dois elétrons fixa a ação efetiva do setor de elétrons a ser independente de calibre. Assim tendo a informação sobre a lagrangiana que gera a função de dois pontos, a lagrangiana efetiva do setor de elétrons 
fica fixada a ser

$$
\mathcal{L}_{F}=m_{F}^{2} \bar{\psi} \int \frac{d \Omega_{d-1}}{\Omega_{d-1}} \frac{i \hat{\phi}}{\hat{Q}^{\alpha} D_{\alpha}} \psi
$$

onde

$$
D_{\alpha}=\partial_{\alpha}-i e A_{\alpha}
$$

é a derivada covariante. 


\section{$5 \quad$ O limite de altas temperaturas em campos de fundo estáticos}

Neste capítulo revisaremos o método do campo de fundo [Abb82 e veremos como, a temperatura finita, a pressão como função do campo externo está relacionada com a ação efetiva térmica como funcional com campo de fundo. Em seguida, veremos que, no limite de altas temperaturas, as funções de Green estáticas podem ser obtidas supondo que todas as componentes do momento externo são nulas, no caso em que os campos externos são bosônicos. Este fato já era conhecido para as funções de dois e três pontos 1PI em ordem de um laço [FPT09]. Recentemente, mostramos que o mesmo vale para a todas amplitudes 1PI em ordem de um laço e para a auto-energia em ordem de dois laços [BS12b]. Apresentaremos esta dedução no presente capítulo e veremos que nosso formalismo é geral o suficiente para tratar as teorias de calibre da QED, Yang-Mills e gravitação. Estes resultados implicam que, no espaço de configurações, podemos obter a ação efetiva estática de um campo bosônico externo calculando diagramas de vácuo para a pressão na presença de um campo externo constante, fato este que será explorado nos dois capítulos que seguem.

\subsection{O método do campo de fundo}

A temperatura finita, o funcional gerador das funções de Green térmicas é a função de partição na presença de uma fonte

$$
Z[J]=\int \mathcal{D} \phi \exp \left\{S_{E}[\phi]+J_{i} \phi^{i}\right\}
$$

onde estamos usando a notação compacta de DeWitt [DeW65],

$$
J_{i} \phi^{i}=\int_{0}^{\beta} d^{d} x J_{A}(x) \phi^{A}(x),
$$


e $A$ é um conjunto de índices sobre graus de liberdade internos arbitrários. O valor esperado no ensemble [veja as equações 2.120) e 2.115] ] será então

$$
\left\langle T_{\tau}\left[\phi^{i_{1}} \cdots \phi^{i_{n}}\right]\right\rangle_{S_{E}}=\left.\frac{1}{Z[0]} \frac{\delta^{n}}{\delta J_{i_{1}} \cdots \delta J_{i_{n}}} Z[J]\right|_{J=0},
$$

onde, na notação de DeWitt, $\phi^{i_{n}}$ representa

$$
\phi^{i_{n}}=\phi^{A_{n}}\left(\vec{x}_{n}, \tau_{n}\right)
$$

As funções de Green conexas (veja a seção 3.3) são geradas pelo funcional

$$
W[J]=\log Z[J]
$$

O campo clássico é definido como o valor esperado

$$
\phi_{c}^{i}=\frac{\delta}{\delta J_{i}} W[J]=\left\langle T_{\tau}\left[\phi^{i}\right]\right\rangle_{J}
$$

onde definimos que

$$
\left\langle T_{\tau}\left[\phi^{i}\right]\right\rangle_{J}=\frac{1}{Z[J]} \int \mathcal{D} \phi \exp \left\{S_{E}+J_{j} \phi^{j}\right\} \phi^{i}
$$

é o valor esperado do campo na presença da fonte $J$. A ação efetiva é um funcional do campo clássico definida como a transformada de Legendre de $W[J]$ na forma

$$
\Gamma\left[\phi_{c}^{i}\right]=W[J]-J_{i} \phi_{c}^{i}
$$

A ação efetiva é o funcional gerador das funções de Green 1PI. Entretanto, a ação efetiva é muito difícil de ser computada diretamente da definição. Veremos agora que um caminho para obter a ação efetiva é o método do campo de fundo.

No método do campo de fundo, vamos supor que o sistema está na presença de um campo puramente clássico $\phi_{E}^{i}$, cujas fontes e a dinâmica são exteriores ao sistema em questão. Neste caso, o funcional gerador das funções de Green se generaliza para

$$
Z\left[J ; \phi_{E}\right]=\int \mathcal{D} \phi \exp \left\{S_{E}\left[\phi+\phi_{E}\right]+J_{i} \phi^{i}\right\}
$$

que é um funcional das fontes e também do campo externo. Fazendo uma mudança de variáveis na integração funcional $\phi \rightarrow \phi+\phi_{E}$, podemos escrever este funcional em termos do funcional (5.1) como

$$
Z\left[J ; \phi_{E}\right]=Z[J] \exp \left(-J_{i} \phi_{E}^{i}\right) .
$$

O funcional gerador das funções de Green conexas também se generaliza de maneira 
natural para

$$
W\left[J ; \phi_{E}\right]=\log Z\left[J ; \phi_{E}\right]=W[J]-J_{i} \phi_{E}^{i} .
$$

O campo clássico na presença do campo externo se modifica para $\bar{\phi}_{c}^{i}$, e é escrito como

$$
\bar{\phi}_{c}^{i}=\frac{\delta}{\delta J_{i}} W\left[J ; \phi_{E}\right]=\phi_{c}^{i}-\phi_{E}^{i}
$$

com $\phi_{c}^{i}$ definido em (5.6). A ação efetiva é então escrita como a transformação de Legendre de $W\left[J ; \phi_{E}\right]$

$$
\Gamma\left[\bar{\phi}_{c}, \phi_{E}\right]=W\left[J ; \phi_{E}\right]-J_{i} \bar{\phi}_{c}^{i}=W[J]-J_{i}\left(\bar{\phi}_{c}^{i}+\phi_{E}^{i}\right)=W[J]-J_{i} \phi_{c}^{i}=\Gamma\left[\phi_{c}\right],
$$

onde usamos (5.11) e 5.12). Substituindo 5.12 em (5.13) obtemos

$$
\Gamma\left[\bar{\phi}_{c}, \phi_{E}\right]=\Gamma\left[\bar{\phi}_{c}+\phi_{E}\right]
$$

que leva à

$$
\Gamma\left[\phi_{E}\right]=\Gamma\left[0, \phi_{E}\right]
$$

Esta equação implica que calculando diagramas de vácuo para $\Gamma\left[0, \phi_{E}\right]$ na presença do campo externo obtemos o funcional gerador das funções de Green 1PI, $\Gamma\left[\phi_{E}\right]$, ou ação efetiva, como funcional do campo de fundo. Assim, calculando contribuições 1PI para a pressão na presença do campo externo, obtemos todas as amplitudes 1PI da teoria.

\subsection{Limite de altas temperaturas das funções de Green estáticas}

Na presença de um campo externo bosônico, podemos mostrar que no limite de altas temperaturas as funções de Green estáticas podem ser obtidas supondo que todas as componentes do momento externo são nulas [FPT09, BS12b]. Nosso argumento será suficientemente geral para englobar as teorias escalar, de Yang-Mills e da Gravitação. Em ordem de um laço faremos um procedimento iterativo para provar esta identidade para todas as funções de Green 1PI, e em ordem de dois laços consideraremos a auto-energia.

\subsubsection{Auto-energia em ordem um laço}

Nesta seção vamos considerar a auto-energia em ordem um laço. Neste caso existem duas topologias que contribuem. Uma delas, que está representada na figura 5.1, é independente do momento externo, sendo trivial a identidade entre a função de Green estática 


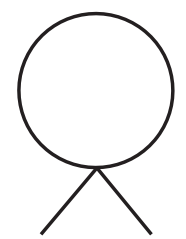

Figura 5.1: Contribuição independente do momento externo para a auto-energia em ordem de um laço.

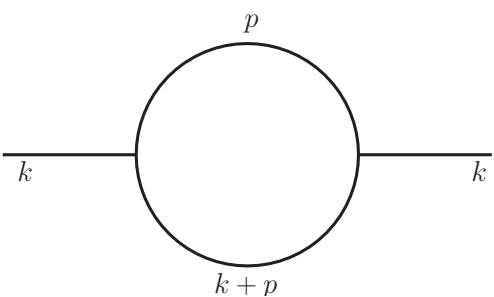

Figura 5.2: Contribuição dependente do momento externo para a auto-energia em ordem de um laço.

e de momento externo nulo. A segunda topologia que contribui para a auto-energia em ordem de um laço está mostrada na figura 5.2. A expressão analítica desse diagrama possui a forma

$$
\Pi=T \sum_{p_{0}} \frac{V\left(p^{\mu}, k^{\mu}\right)}{\left(p_{0}^{2}-\vec{p}^{2}\right)\left[\left(p_{0}-k_{0}\right)^{2}-(\vec{p}-\vec{k})^{2}\right]},
$$

onde estaremos omitindo a integral na parte espacial do momento, pois ela não será relevante para os argumentos que seguem. A função $V$ representa componentes tensoriais que dependem do tipo de vértices da teoria. A componente temporal do momento possui a forma $p_{0}=i \omega_{n}^{B / F}$, onde $\omega_{n}^{B / F}$ é uma frequência de Matsubara de um bóson ou férmion, dependendo da paridade da respectiva linha. Assim, usando os resultado do apêndice C, a soma de Matsubara pode ser escrita como

$$
T \sum_{p_{0}=i \omega_{n}^{B / F}}=\oint_{C} \frac{d p_{0}}{2 \pi i} \operatorname{coth}^{ \pm 1}\left(\beta p_{0} / 2\right),
$$

onde o contorno $C=C_{1} \cup C_{2}$ está representado na figura 5.3 (a). Para a parte dependente da temperatura, podemos fazer

$$
\operatorname{coth}^{ \pm 1}\left(\beta p_{0} / 2\right) \rightarrow \pm 2 N_{B / F}\left(p_{0}\right)
$$

onde $N_{B / F}$ representa as distribuições térmicas de Bose-Einstein e Fermi-Dirac, respectivamente. 
No limite estático $\left(k_{0}=0\right)$, a amplitude $(5.16)$ pode ser reescrita de maneira compacta como

$$
\Pi_{e}=\oint_{C} \frac{d p_{0}}{2 \pi i} \frac{f\left(p_{0}\right)}{\left(p_{0}^{2}-x^{2}\right)\left(p_{0}^{2}-w^{2}\right)},
$$

onde $x=|\vec{p}|$ e $w=|\vec{p}+\vec{k}|$.

Considerando apenas a parte dependente da temperatura, o numerador $f\left(p_{0}\right)$ possui a forma

$$
f\left(p_{0}\right)=N_{p}\left(p_{0}\right) V\left(p^{\mu}\right)
$$

Enfatizando, $N_{p}\left(p_{0}\right)$ é a função de distribuição de Fermi-Dirac ou Bose-Einstein, da paridade da linha de momento $p$. No contorno $C_{2}$ é conveniente escrever $f\left(p_{0}\right) \rightarrow$ $-N_{p}\left(-p_{0}\right) V\left(p^{\mu}\right)$, que é válido para os termos dependentes da temperatura, isto é, efetivamente temos a igualdade

$$
f\left(p_{0}\right)=\operatorname{sgn}\left(p_{0}\right) N_{p}\left(\left|p_{0}\right|\right) V\left(p^{\mu}\right),
$$

onde sgn representa a função sinal.

A função $V\left(p^{\mu}\right)$ depende do modelo em questão e, em geral, depende do quadrimomento externo $k$. Para as teorias de calibre da QED, de Yang-Mills ou gravitação, as componentes de $V$ são polinômios nas componentes de $p$ e $k$. Por contagem de potências, os termos proporcionais ao momento externo no numerador serão subdominantes no limite de altas temperaturas. Então, podemos fazer $k=0$ em $V$ [Bel96, KG06]. Nos argumentos que seguem, apenas será explicitada a dependência em $f$ na componente temporal do momento $p_{0}$.

Usando o comportamento assintótico de $N_{p}\left(p_{0}\right)$, podemos fechar o contorno de integração $C$ no infinito como mostrado na figura 5.3 (b), independentemente do comportamento assintótico da função $V\left(p^{\mu}\right)$, pois este será polinomial.

Portanto, pelo teorema do resíduo teremos

$$
\begin{aligned}
\Pi_{e} & =-\left[\frac{f(x)}{2 x\left(x^{2}-w^{2}\right)}+(x \rightarrow-x)+\frac{f(w)}{2 w\left(w^{2}-x^{2}\right)}+(w \rightarrow-w)\right] \\
& =-\frac{1}{x^{2}-w^{2}}\left[\frac{f(x)}{2 x}-\frac{f(w)}{2 w}+(x, w \rightarrow-x,-w)\right] .
\end{aligned}
$$

No limite de altas temperaturas, a amplitude será dominada pela região em que o momento externo é muito maior que o momento de integração, ou seja região dos "Hard Thermal Loops" (HTL) [Bel96, KG06]. Assim podemos escrever $w=x+\epsilon$, onde $\epsilon=w-x \ll x$, e fazer uma expansão em torno de $\epsilon=0$. Procedendo desta maneira, vemos que o termo 


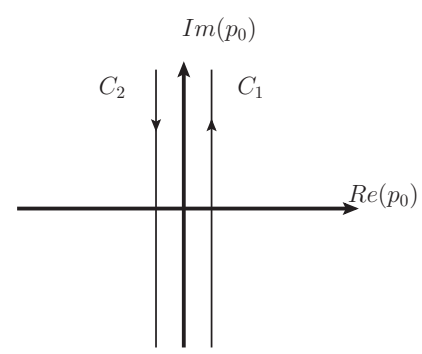

(a)

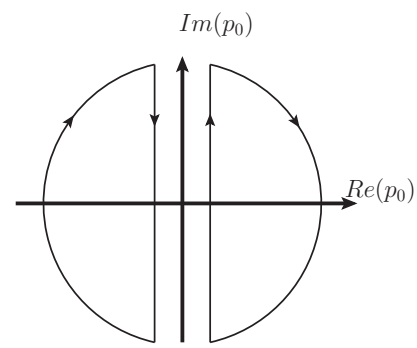

(b)

Figura 5.3: Contorno de integração no plano complexo $p_{0}$.

dominante tem a forma

$$
\frac{1}{x^{2}-w^{2}}\left[\frac{f(x)}{2 x}-\frac{f(w)}{2 w}\right] \simeq \frac{1}{2 x} \frac{d}{d x} \frac{f(x)}{2 x} .
$$

Isto fornece a contribuição dominante no limite de altas temperaturas da auto-energia estática como sendo

$$
\Pi_{e}=-\left[\frac{1}{2 x} \frac{d}{d x} \frac{f(x)}{2 x}+(x \rightarrow-x)\right]
$$

Já no caso de momento externo nulo, a auto-energia assume a forma

$$
\Pi_{0}=\oint_{C} \frac{d p_{0}}{2 \pi i} \frac{f\left(p_{0}\right)}{\left(p_{0}^{2}-x^{2}\right)^{2}}
$$

Usando o teorema dos resíduos, podemos novamente integrar $p_{0}$. Para tanto, devemos notar que aqui $x$ será um polo duplo, de modo que a integral fornece

$$
\Pi_{0}=-\left[\lim _{p_{0} \rightarrow x} \frac{d}{d p_{0}} \frac{f\left(p_{0}\right)}{\left(p_{0}+x\right)^{2}}+(x \rightarrow-x)\right]
$$

e avaliando o limite teremos

$$
\lim _{p_{0} \rightarrow x} \frac{d}{d p_{0}} \frac{f\left(p_{0}\right)}{\left(p_{0}+x\right)^{2}}=\frac{1}{4 x^{2}} \frac{d}{d x} f(x)-\frac{f(x)}{4 x^{3}}=\frac{1}{2 x} \frac{d}{d x} \frac{f(x)}{2 x} .
$$


Assim, no limite de momento externo nulo a auto-energia assumi a forma

$$
\Pi_{0}=-\left[\frac{1}{2 x} \frac{d}{d x} \frac{f(x)}{2 x}+(x \rightarrow-x)\right]
$$

que é idêntico ao limite estático (5.24). Isto mostra que para a topologia mostrada na figura 5.2 podemos obter o limite estático supondo que todas as componentes do momento externo são nulas, no limite de altas temperaturas. Levando em conta o diagrama independente do momento externo, representado na figura 5.1. vemos que o mesmo vale em geral para a auto-energia em ordem um laço.

Para os diagramas mostrados na figura 5.6 como os laços não se sobrepõem, a mesma identidade segue de uma dedução análoga, pois nestes diagramas as integrais dos laços serão independentes.

\subsubsection{Procedimento iterativo para amplitudes em um laço}

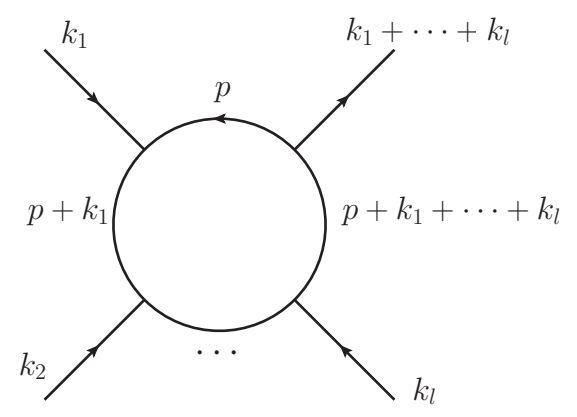

Figura 5.4: Amplitude 1PI com $l+1$ vértices. Está implícito que algumas das linhas externas podem representar um conjunto de linhas entrando no mesmo vértice.

Vamos considerar uma função de Green 1PI em ordem um laço, com um número arbitrário de linhas externas. A figura 5.4 mostra um diagrama contendo $l+1$ vértices. Para uma topologia geral, $k_{i}$ denota a soma $\operatorname{dos} n_{i}=1,2 \ldots$ quadri-momentos associados com as $n_{i}$ linhas inseridas no vértice $\left(n_{i}+2\right)$ pontos, de modo que o número total de linhas externas ao diagrama é $\sum_{i} n_{i} \geq l+1$.

No limite estático, quando a componente temporal de todos os quadri-momentos externos se anula $\left(k_{0 i}=0\right)$, depois de transformarmos a soma de Matsubara em uma integral usando (5.17), esta amplitude pode ser escrita como

$$
\mathcal{A}_{e}^{l+1}=\oint_{C} \frac{d p_{0}}{2 \pi i} \frac{\bar{f}\left(p_{0}\right)}{\left(p_{0}^{2}-x^{2}\right)} \prod_{n=1}^{l} \frac{1}{p_{0}^{2}-w_{n}^{2}},
$$


onde estamos usando a notação

$$
w_{n}=\left|\vec{p}+\vec{k}_{1}+\cdots+\vec{k}_{n}\right|
$$

e $x=|\vec{p}|$. No limite de altas temperaturas a função $\bar{f}\left(p_{0}\right)$ possui propriedades semelhantes a $f\left(p_{0}\right)$ na equação 5.20 , sendo o produto de uma distribuição térmica com um tensor independente do momento externo.

Fazendo a integral em $p_{0}$ com a ajuda do teorema dos resíduos, usando o contorno de integração mostrado na figura 5.3 (b), obtemos

$$
\begin{aligned}
\mathcal{A}_{e}^{l+1} & =-\left\{\frac{\bar{f}(x)}{2 x} \prod_{n=1}^{l} \frac{1}{x^{2}-w_{n}^{2}}+\sum_{m=1}^{l} \frac{\bar{f}\left(w_{m}\right)}{2 w_{m}\left(w_{m}^{2}-x^{2}\right)} \prod_{\substack{n=1 \\
n \neq m}}^{l} \frac{1}{w_{m}^{2}-w_{n}^{2}}\right\} \\
& +\left\{\left(x, w_{j}\right) \rightarrow\left(-x,-w_{j}\right)\right\}, \quad j=1, \cdots, l .
\end{aligned}
$$

Para obter o limite de altas temperaturas desta amplitude, vamos considerar um procedimento iterativo. Na região dos HTL, todos os momentos externos $\vec{k}_{i}$ são muito menores que o quadri-momento $p$. A fim de obter a aproximação HTL, nossa estratégia será fazer um procedimento gradual considerando cada momento externo $\vec{k}_{i}$ pequeno comparado com $\vec{p}$, construindo assim um raciocínio indutivo.

Vamos primeiro explicitar a dependência em $w_{1}$, de modo que (5.31) pode ser reescrita como

$$
\begin{aligned}
\mathcal{A}_{e}^{l+1} & =-\left\{\frac{1}{x^{2}-w_{1}^{2}}\left(\frac{\bar{f}(x)}{2 x} \prod_{n=2}^{l} \frac{1}{x^{2}-w_{n}^{2}}-\frac{\bar{f}\left(w_{1}\right)}{2 w_{1}} \prod_{n=2}^{l} \frac{1}{w_{1}^{2}-w_{n}^{2}}\right)\right. \\
& \left.+\sum_{m=2}^{l} \frac{\bar{f}\left(w_{m}\right)}{2 w_{m}\left(w_{m}^{2}-x^{2}\right)\left(w_{m}^{2}-w_{1}^{2}\right)} \prod_{\substack{n=2 \\
n \neq m}}^{l} \frac{1}{w_{m}^{2}-w_{n}^{2}}\right\} \\
& +\left\{\left(x, w_{j}\right) \rightarrow\left(-x,-w_{j}\right)\right\} \quad j=1, \cdots, l
\end{aligned}
$$

Agora vamos considerar o momento $\vec{k}_{1}$ muito menor que o momento do laço $\vec{p}$, de modo que, procedendo como na sub-seção anterior, nos podemos escrever $w_{1}=x+\epsilon$ e expandir 
em torno de $\epsilon=0$. Isto fornece

$$
\begin{aligned}
\mathcal{A}_{e}^{l+1} & \simeq-\left\{\frac{1}{2 x} \frac{\partial}{\partial x}\left(\frac{\bar{f}(x)}{2 x} \prod_{n=2}^{l} \frac{1}{x^{2}-\bar{w}_{n}^{2}}\right)+\sum_{m=2}^{l} \frac{\bar{f}\left(\bar{w}_{m}\right)}{2 \bar{w}_{m}\left(\bar{w}_{m}^{2}-x^{2}\right)^{2}} \prod_{\substack{n=2 \\
n \neq m}}^{l} \frac{1}{\bar{w}_{m}^{2}-\bar{w}_{n}^{2}}\right\} \\
& +\left\{\left(x, \bar{w}_{j}\right) \rightarrow\left(-x,-\bar{w}_{j}\right)\right\}, \quad j=2, \cdots, l, \\
& =-\frac{1}{2 x} \frac{\partial}{\partial x}\left\{\frac{\bar{f}(x)}{2 x} \prod_{n=2}^{l} \frac{1}{x^{2}-\bar{w}_{n}^{2}}+\sum_{m=2}^{l} \frac{\bar{f}\left(\bar{w}_{m}\right)}{2 \bar{w}_{m}\left(\bar{w}_{m}^{2}-x^{2}\right)} \prod_{\substack{n=2 \\
n \neq m}}^{l} \frac{1}{\bar{w}_{m}^{2}-\bar{w}_{n}^{2}}\right\} \\
& +\left\{\left(x, \bar{w}_{j}\right) \rightarrow\left(-x,-\bar{w}_{j}\right)\right\} \quad j=2 \ldots l,
\end{aligned}
$$

com a notação

$$
\bar{w}_{n}=\left|\vec{p}+\vec{k}_{2}+\cdots+\vec{k}_{n}\right|, \quad n=2, \cdots, l .
$$

Usando o teorema do resíduo podemos escrever (5.33) como

$$
\begin{aligned}
\mathcal{A}_{e}^{l+1} & \simeq \frac{1}{2 x} \frac{\partial}{\partial x} \oint_{C} \frac{d p_{0}}{2 \pi i} \frac{\bar{f}\left(p_{0}\right)}{\left(p_{0}^{2}-x^{2}\right)} \prod_{n=2}^{l} \frac{1}{p_{0}^{2}-\bar{w}_{n}^{2}} \\
& =\oint_{C} \frac{d p_{0}}{2 \pi i} \frac{\bar{f}\left(p_{0}\right)}{\left(p_{0}^{2}-x^{2}\right)^{2}} \prod_{n=2}^{l} \frac{1}{p_{0}^{2}-\bar{w}_{n}^{2}},
\end{aligned}
$$

onde a equivalência com 5.33 pode ser verificada computando e integral de resíduo na primeira linha de (5.35).

Neste ponto, note que se fizermos $\vec{k}_{1}=0$ na amplitude estática 5.29 , iremos obter o mesmo resultado 5.35). Portanto, este é um caso especial da identidade entre a função de Green estática e de momento externo nulo e será a base da indução. Note que, no caso particular em que $l=1$, as equações 5.29$)\left(\operatorname{com} \vec{k}_{1}=0\right)$ e 5.35 verificam explicitamente esta identidade para a auto-energia, como feito na sub-seção anterior.

Para completar o raciocínio indutivo, vamos supor agora que a amplitude, com $\vec{k}_{1}$, $\cdots, \vec{k}_{n-1}$ muito menor que o momento do laço $\vec{p}$, pode ser aproximada por

$$
\mathcal{A}_{e}^{l+1} \simeq \oint_{C} \frac{d p_{0}}{2 \pi i} \frac{\bar{f}\left(p_{0}\right)}{\left(p_{0}^{2}-x^{2}\right)^{n}} \prod_{i=n}^{l} \frac{1}{p_{0}^{2}-v_{i}^{2}},
$$


onde

$$
v_{i}=\left|\vec{p}+\vec{k}_{n}+\cdots+\vec{k}_{i}\right| \quad i=n, \cdots, l,
$$

e notamos que (5.36) é a amplitude (5.29) com os momentos $\vec{k}_{1}, \cdots, \vec{k}_{n-1}$ nulos. Logo, partindo de (5.36), vamos provar que, quando o momento $\vec{k}_{n}$ é também muito menor que $\vec{p}$, obtemos (5.36) com $n \rightarrow n+1$ [veja equações (5.51) e (5.54)], isto é, com $\vec{k}_{n}$ nulo.

Usando o teorema dos resíduos e notando que o ponto $p_{0}=x$ é um polo de ordem $n$, vemos que a integração em $p_{0}$ na equação 5.36 fornece

$$
\begin{aligned}
\mathcal{A}_{e}^{l+1} & \simeq-\left\{\lim _{p_{0} \rightarrow x} \frac{1}{(n-1) !}\left(\frac{\partial}{\partial p_{0}}\right)^{n-1} \frac{\bar{f}\left(p_{0}\right)}{\left(p_{0}+x\right)^{n}} \prod_{i=n}^{l} \frac{1}{p_{0}^{2}-v_{i}^{2}}\right. \\
& \left.+\sum_{j=n}^{l} \frac{\bar{f}\left(v_{j}\right)}{2 v_{j}\left(v_{j}^{2}-x^{2}\right)^{n}} \prod_{\substack{i=n \\
i \neq j}}^{l} \frac{1}{v_{j}^{2}-v_{i}^{2}}\right\}+\left\{\left(x, v_{m}\right) \rightarrow\left(-x,-v_{m}\right)\right\}, m=2, \cdots, l, \\
& =-\left\{\lim _{p_{0} \rightarrow x} \frac{1}{(n-1) !}\left(\frac{\partial}{\partial p_{0}}\right)^{n-1} \frac{\bar{f}\left(p_{0}\right)}{\left(p_{0}+x\right)^{n}\left(p_{0}^{2}-v_{n}^{2}\right)} \prod_{i=n+1}^{l} \frac{1}{p_{0}^{2}-v_{i}^{2}}\right. \\
& \left.+\frac{\bar{f}\left(v_{n}\right)}{2 v_{n}\left(v_{n}^{2}-x^{2}\right)^{n}} \prod_{i=n+1}^{l} \frac{1}{v_{n}^{2}-v_{i}^{2}}+\sum_{j=n+1}^{l} \frac{v_{j}}{2 v_{j}\left(v_{j}^{2}-x^{2}\right)^{n}\left(v_{j}^{2}-v_{n}^{2}\right)} \prod_{i=n+1}^{l} \frac{1}{v_{j}^{2}-v_{i}^{2}}\right\} \\
& +\left\{\left(x, v_{m}\right) \rightarrow\left(-x,-v_{m}\right)\right\} m=n, \cdots, l .
\end{aligned}
$$

O primeiro termo em 5.38 pode ser escrito como

$$
\begin{aligned}
T_{1} & =\lim _{p_{0} \rightarrow x} \frac{1}{(n-1) !}\left(\frac{\partial}{\partial p_{0}}\right)^{n-1} \frac{h\left(p_{0}\right)}{\left(p_{0}+x\right)^{n}\left(p_{0}^{2}-v_{n}^{2}\right)} \\
& =\lim _{p_{0} \rightarrow x} \frac{1}{(n-1) !}\left(\frac{\partial}{\partial p_{0}}\right)^{n-1} \frac{h\left(p_{0}\right)}{\left(p_{0}+x\right)^{n}\left(p_{0}+v_{n}\right)\left(p_{0}-v_{n}\right)}
\end{aligned}
$$

onde introduzimos

$$
h\left(p_{0}\right)=\bar{f}\left(p_{0}\right) \prod_{i=n+1}^{l} \frac{1}{p_{0}^{2}-v_{i}^{2}}
$$

Usando a regra de Leibniz, temos uma analogia com a formula binomial na forma

$$
\left(\frac{\partial}{\partial p_{0}}\right)^{n-1}\left[A\left(p_{0}\right) B\left(p_{0}\right)\right]=\sum_{t=0}^{n-1} \frac{(n-1) !}{t !(n-1-t) !}\left[\left(\frac{\partial}{\partial p_{0}}\right)^{t} A\left(p_{0}\right)\right]\left[\left(\frac{\partial}{\partial p_{0}}\right)^{n-1-t} B\left(p_{0}\right)\right]
$$


Substituindo 5.41 em (5.39) temos

$$
T_{1}=\sum_{t=0}^{n-1} \frac{1}{t !(n-1-t) !} \lim _{p_{0} \rightarrow x}\left[\left(\frac{\partial}{\partial p_{0}}\right)^{n-1-t} \frac{1}{p_{0}-v_{n}}\right]\left[\left(\frac{\partial}{\partial p_{0}}\right)^{t} \frac{h\left(p_{0}\right)}{\left(p_{0}+x\right)^{n}\left(p_{0}+v_{n}\right)}\right]
$$

e calculando explicitamente a derivada teremos

$$
\begin{aligned}
T_{1} & =\sum_{t=0}^{n-1} \frac{1}{t !(n-1-t) !} \lim _{p_{0} \rightarrow x}\left[\frac{(-1)^{n-t-1}(n-t-1) !}{\left(p_{0}-v_{n}\right)^{n-t}}\right]\left[\left(\frac{\partial}{\partial p_{0}}\right)^{t} \frac{h\left(p_{0}\right)}{\left(p_{0}+x\right)^{n}\left(p_{0}+v_{n}\right)}\right] \\
& =\sum_{t=0}^{n-1} \frac{1}{t !} \lim _{p_{0} \rightarrow x}\left[\frac{-1}{\left(v_{n}-p_{0}\right)^{n-t}}\right]\left[\left(\frac{\partial}{\partial p_{0}}\right)^{t} \frac{h\left(p_{0}\right)}{\left(p_{0}+x\right)^{n}\left(p_{0}+v_{n}\right)}\right] \\
& =\sum_{t=0}^{n-1} \frac{1}{t !} \frac{-1}{\left(v_{n}-x\right)^{n-t}} \lim _{p_{0} \rightarrow x}\left(\frac{\partial}{\partial p_{0}}\right)^{t} \frac{h\left(p_{0}\right)}{\left(p_{0}+x\right)^{n}\left(p_{0}+v_{n}\right)} .
\end{aligned}
$$

Agora, no limite de altas temperaturas, quando o momento $\vec{k}_{n}$ é muito menor que o momento de integração $\vec{p}$, escrevemos $v_{n}=x+\epsilon$. Assim, o termo dominante na equação 5.43 é

$$
T_{1} \simeq-\sum_{t=0}^{n-1} \frac{1}{t !\left(v_{n}-x\right)^{n-t}} \lim _{p_{0} \rightarrow x}\left(\frac{\partial}{\partial p_{0}}\right)^{t} \frac{h\left(p_{0}\right)}{\left(p_{0}+x\right)^{n+1}}
$$

De maneira similar, usando a equação 5.40 , o segundo termo na equação 5.38 pode ser escrito como

$$
T_{2}=\frac{1}{\left(v_{n}-x\right)^{n}} \frac{h\left(v_{n}\right)}{2 v_{n}\left(v_{n}+x\right)^{n}} .
$$

No limite de altas temperaturas este termo pode ser aproximado por

$$
T_{2} \simeq \frac{1}{\left(v_{n}-x\right)^{n}} \frac{h\left(v_{n}\right)}{\left(v_{n}+x\right)^{n+1}} .
$$

Fazendo uma expansão em série de Taylor, temos

$$
T_{2} \simeq \frac{1}{\left(v_{n}-x\right)^{n}} \sum_{t=0}^{\infty} \frac{\left(v_{n}-x\right)^{t}}{t !} \lim _{v_{n} \rightarrow x}\left(\frac{\partial}{\partial v_{n}}\right)^{t} \frac{h\left(v_{n}\right)}{\left(v_{n}+x\right)^{n+1}}
$$

que pode ser reescrito como

$$
T_{2} \simeq \sum_{t=0}^{\infty} \frac{1}{t !\left(v_{n}-x\right)^{n-t}} \lim _{p_{0} \rightarrow x}\left(\frac{\partial}{\partial p_{0}}\right)^{t} \frac{h\left(p_{0}\right)}{\left(p_{0}+x\right)^{n+1}}
$$


Combinando as equações (5.44) e (5.48), teremos

$$
T_{1}+T_{2} \simeq \sum_{t=n}^{\infty} \frac{1}{t !\left(v_{n}-x\right)^{n-t}} \lim _{p_{0} \rightarrow x}\left(\frac{\partial}{\partial p_{0}}\right)^{t} \frac{h\left(p_{0}\right)}{\left(p_{0}+x\right)^{n+1}},
$$

e, por contagem de potências, o primeiro termo será o termo dominante no limite de altas temperaturas. Logo, ignorando todas as contribuições sub-dominantes no limite de altas temperaturas, nos obtemos

$$
\begin{aligned}
T_{1}+T_{2} & \simeq \frac{1}{n !} \lim _{p_{0} \rightarrow x}\left(\frac{\partial}{\partial p_{0}}\right)^{n} \frac{h\left(p_{0}\right)}{\left(p_{0}+x\right)^{n+1}} \\
& \simeq \frac{1}{n !} \lim _{p_{0} \rightarrow x}\left(\frac{\partial}{\partial p_{0}}\right)^{n} \frac{\bar{f}\left(p_{0}\right)}{\left(p_{0}+x\right)^{n+1}} \prod_{i=n+1}^{l} \frac{1}{p_{0}^{2}-\bar{v}_{i}^{2}}
\end{aligned}
$$

onde

$$
\bar{v}_{i}=\left|\vec{p}+\vec{k}_{n+1}+\cdots+\vec{k}_{i}\right|, i=n+1, \cdots, l
$$

O último termo na equação (5.38) possui e seguinte forma no limite de altas temperaturas

$$
T_{3} \simeq \sum_{j=n+1}^{l} \frac{\bar{f}\left(\bar{v}_{j}\right)}{2 \bar{v}_{j}\left(\bar{v}_{j}^{2}-x^{2}\right)^{n+1}} \prod_{\substack{i=n+1 \\ i \neq j}}^{l} \frac{1}{\bar{v}_{j}^{2}-\bar{v}_{i}^{2}}
$$

Combinando as equações 5.50 e 5.52 , obtemos

$$
\begin{aligned}
\mathcal{A}_{e}^{l+1} & \simeq-\left\{\frac{1}{n !} \lim _{p_{0} \rightarrow x}\left(\frac{\partial}{\partial p_{0}}\right)^{n} \frac{\bar{f}\left(p_{0}\right)}{\left(p_{0}+x\right)^{n+1}} \prod_{i=n+1}^{l} \frac{1}{p_{0}^{2}-\bar{v}_{i}^{2}}\right. \\
& \left.+\sum_{j=n+1}^{l} \frac{\bar{f}\left(\bar{v}_{j}\right)}{2 \bar{v}_{j}\left(\bar{v}_{j}^{2}-x^{2}\right)^{n+1}} \prod_{\substack{i=n+1 \\
i \neq j}}^{l} \frac{1}{\bar{v}_{j}^{2}-\bar{v}_{i}^{2}}\right\} \\
& +\left\{\left(x, \bar{v}_{m}\right) \rightarrow\left(-x,-\bar{v}_{m}\right)\right\} \quad m=n+1, \ldots, l .
\end{aligned}
$$

Finalmente usando o teorema dos resíduos teremos

$$
\mathcal{A}_{e}^{l+1} \simeq \oint_{C} \frac{d p_{0}}{2 \pi i} \frac{\bar{f}\left(p_{0}\right)}{\left(p_{0}^{2}-x^{2}\right)^{n+1}} \prod_{i=n+1}^{l} \frac{1}{p_{0}^{2}-\bar{v}_{i}^{2}},
$$

que é a amplitude (5.36) com $p_{n}=0$, o que completa o raciocínio indutivo para todas as amplitudes 1PI na região dos HTL. 
Uma consequência direta da prova anterior é que podemos escrever

$$
\mathcal{A}_{e}^{l+1} \simeq \oint_{C} \frac{d p_{0}}{2 \pi i} \frac{\bar{f}\left(p_{0}\right)}{\left(p_{0}^{2}-x^{2}\right)^{l+1}}=\mathcal{A}_{0}^{l+1},
$$

o que generaliza o resultado obtido em [FPT09]. Logo, podemos escrever uma amplitude 1PI estática em ordem um laço, no limite de altas temperaturas, como segue

$$
\begin{aligned}
\mathcal{A}_{e}^{l+1} & \simeq \frac{1}{l !}\left(\frac{1}{2 x} \frac{\partial}{\partial x}\right)^{l} \oint_{C} \frac{d p_{0}}{2 \pi i} \frac{\bar{f}\left(p_{0}\right)}{p_{0}^{2}-x^{2}} \\
& =\frac{1}{l !}\left(\frac{1}{2 x} \frac{\partial}{\partial x}\right)^{l} \frac{\bar{f}(x)}{2 x}+(x \rightarrow-x)
\end{aligned}
$$

\subsection{Auto-energia em ordem dois laços}
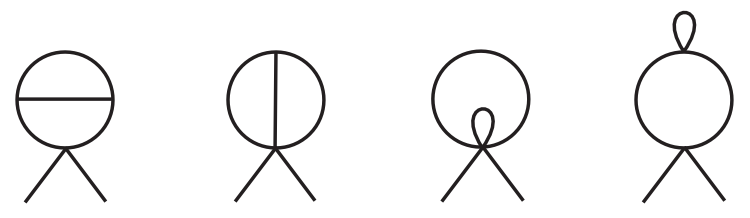

Figura 5.5: Topologias independentes do momento em ordem de dois laços.

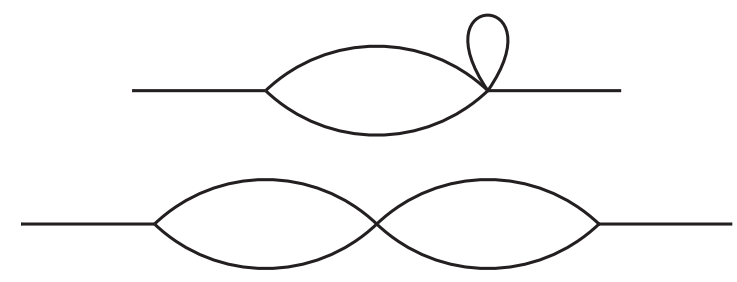

Figura 5.6: Topologias que podem ser reduzidas ao caso de um laço.

Nesta seção vamos considerar as contribuições em ordem de dois laços para a autoenergia. As topologias mostradas na figura 5.5 são independente do momento externo, de modo que a identidade entre as funções de Green estática e de momento externo nulo no limite de altas temperaturas é trivial. Já para as topologias mostradas na figura 5.6

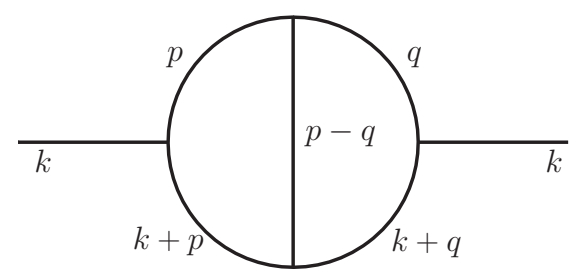

Figura 5.7: Primeira topologia para a auto-energia em ordem dois laços. 
os laços não se sobrepõe e a dedução desta identidade segue os mesmos passos da autoenergia em ordem de um laço. Nesta seção levaremos em conta as outras topologias que são dependentes do momento externo e não podem ser reduzidas ao caso de um laço. Existem cinco topologias deste tipo. Nós vamos apresentar aqui os detalhes de duas delas, que representam todas as técnicas necessárias e ilustram os aspectos principais da nossa analise. As outras três topologias serão consideradas no apêndice E.

\subsubsection{Primeira topologia}

Vamos primeiro considerar a contribuição mostrada na figura 5.7. No limite estático a amplitude pode ser escrita como

$$
\Pi_{e}^{A}=\oint_{C} \frac{d p_{0}}{2 \pi i} \oint_{C} \frac{d q_{0}}{2 \pi i} \frac{f^{A}\left(p_{0}, q_{0}\right)}{\left(p_{0}^{2}-x^{2}\right)\left(p_{0}^{2}-w^{2}\right)\left[\left(p_{0}-q_{0}\right)^{2}-z^{2}\right]\left(q_{0}^{2}-y^{2}\right)\left(q_{0}^{2}-v^{2}\right)},
$$

onde $x=|\vec{p}|, y=|\vec{q}|, w=|\vec{p}+\vec{k}|, v=|\vec{q}+\vec{k}|$ e $z=|\vec{p}-\vec{q}|$. Para a contribuição dominante no limite de altas temperaturas, precisamos apenas considerar as contribuições que envolvem o produto de duas distribuições térmicas, de modo que a função $f^{A}\left(p_{0}, q_{0}\right)$ possui a forma

$$
f^{A}\left(p_{0}, q_{0}\right)=N_{p}\left(p_{0}\right) N_{q}\left(q_{0}\right) V\left(p^{\mu}, q^{\nu}\right)
$$

e as mesmas considerações feitas sobre o limite de altas temperaturas da função $f\left(p_{0}\right)$, na seção 5.2.1. também vale para $f^{A}\left(p_{0}, q_{0}\right)$. Logo, no limite de altas temperaturas, $V$ será um tensor cujas componentes são polinômios que não dependem do momento externo.

Usando o teorema dos resíduos para avaliar a integral em $p_{0}$, obtemos

$$
\begin{aligned}
\Pi_{e}^{A} & =-\oint_{C} \frac{d q_{0}}{2 \pi i}\left\{\frac{f^{A}\left(x, q_{0}\right)}{2 x\left(x^{2}-w^{2}\right)\left[\left(x-q_{0}\right)^{2}-z^{2}\right]\left(q_{0}^{2}-y^{2}\right)\left(q_{0}^{2}-v^{2}\right)}\right. \\
& +\frac{f^{A}\left(w, q_{0}\right)}{2 w\left(w^{2}-x^{2}\right)\left[\left(w-q_{0}\right)^{2}-z^{2}\right]\left(q_{0}^{2}-y^{2}\right)\left(q_{0}^{2}-v^{2}\right)} \\
& \left.+\frac{f^{A}\left(q_{0}+z, q_{0}\right)}{\left.\left.2 z\left[\left(q_{0}+z\right)^{2}-x^{2}\right)\right]\left[\left(q_{0}+z\right)^{2}-w^{2}\right)\right]\left(q_{0}^{2}-y^{2}\right)\left(q_{0}^{2}-v^{2}\right)}\right\} \\
& +\{(x, w, z) \rightarrow(-x,-w,-z)\} .
\end{aligned}
$$


Similarmente, a integração em $q_{0}$ fornece

$$
\begin{aligned}
\Pi_{e}^{A} & =\left\{\left[\frac{f^{A}(x, x+z)}{4 z x\left(x^{2}-w^{2}\right)\left[(x+z)^{2}-y^{2}\right]\left[(x+z)^{2}-v^{2}\right]}\right.\right. \\
& \left.+\frac{f^{A}(w, w+z)}{4 z w\left(w^{2}-x^{2}\right)\left[(w+z)^{2}-y^{2}\right]\left[(w+z)^{2}-v^{2}\right]}+(z \rightarrow-z)\right] \\
& +\left[\frac{f^{A}(x, y)}{4 x y\left(x^{2}-w^{2}\right)\left[(x-y)^{2}-z^{2}\right]\left(y^{2}-v^{2}\right)}+\frac{f^{A}(w, y)}{4 w y\left(w^{2}-x^{2}\right)\left[(w-y)^{2}-z^{2}\right]\left(y^{2}-v^{2}\right)}\right. \\
& \left.+\frac{f^{A}(y+z, y)}{4 z y\left[(y+z)^{2}-x^{2}\right]\left[(y+z)^{2}-w^{2}\right]\left(y^{2}-v^{2}\right)}+(y \rightarrow-y)\right] \\
& +\left[\frac{f^{A}(x, v)}{4 x v\left(x^{2}-w^{2}\right)\left[(x-v)^{2}-z^{2}\right]\left(v^{2}-y^{2}\right)}+\frac{f^{A}(v+z, v)}{4 w v(w)}\right] \\
& \left.+\frac{f^{A}(x, x-z)}{4 z v\left[(v+z)^{2}-x^{2}\right]\left[(v+z)^{2}-w^{2}\right]\left(v^{2}-y^{2}\right)}+(v \rightarrow-v)\right] \\
& +\left[\frac{f^{A}(w, w-z)}{4 z x\left(x^{2}-w^{2}\right)\left[(x-z)^{2}-y^{2}\right]\left[(x-z)^{2}-v^{2}\right]}+(x \rightarrow-x)\right] \\
& \left.+\left[\frac{\left.y^{2}\right)}{4 z w\left(w^{2}-x^{2}\right)\left[(w-z)^{2}-y^{2}\right]\left[(w-z)^{2}-v^{2}\right]}+(w \rightarrow-w)\right]\right\} \\
& +\{[(x, w, z) \rightarrow(-x,-w,-z)]\} .
\end{aligned}
$$

A região dos HTL é caracterizada por $q \gg k$ e $p \gg k$, e portanto escrevemos $w=x+\epsilon$ e $v=y+\delta, \operatorname{com} \epsilon=w-x \ll x$ e $\delta=v-y \ll y$. Fazendo uma expansão em série em torno de $\epsilon=0$ e $\delta=0$, todos os termos na equação (5.60) podem ser tratados usando as expressões que seguem

$$
\begin{aligned}
& \frac{1}{x^{2}-w^{2}}\left[\frac{f^{A}(x, x+z)}{4 z x\left[(x+z)^{2}-y^{2}\right]\left[(x+z)^{2}-v^{2}\right]}-\frac{f^{A}(w, w+z)}{4 z w\left[(w+z)^{2}-y^{2}\right]\left[(w+z)^{2}-v^{2}\right]}\right] \\
& \simeq \frac{1}{2 x} \frac{\partial}{\partial x}\left[\frac{f^{A}(x, x+z)}{4 z x\left[(x+z)^{2}-y^{2}\right]^{2}}\right], \\
& \frac{1}{y^{2}-v^{2}}\left[\frac{f^{A}(y+z, y)}{4 z y\left[(y+z)^{2}-x^{2}\right]\left[(y+z)^{2}-w^{2}\right]}-\frac{f^{A}(v+z, v)}{4 z v\left[(v+z)^{2}-x^{2}\right]\left[(v+z)^{2}-w^{2}\right]}\right] \\
& \simeq \frac{1}{2 y} \frac{\partial}{\partial y}\left[\frac{f^{A}(y+z, y)}{4 z y\left[(y+z)^{2}-x^{2}\right]^{2}}\right], \\
& \frac{1}{x^{2}-w^{2}}\left[\frac{f^{A}(x, x-z)}{4 z x\left[(x-z)^{2}-y^{2}\right]\left[(x-z)^{2}-v^{2}\right]}-\frac{f^{A}(w, w-z)}{4 z w\left[(w-z)^{2}-y^{2}\right]\left[(w-z)^{2}-v^{2}\right]}\right] \\
& \simeq \frac{1}{2 x} \frac{\partial}{\partial x}\left[\frac{f^{A}(x, x-z)}{4 z x\left[(x-z)^{2}-y^{2}\right]^{2}}\right],
\end{aligned}
$$




$$
\begin{aligned}
\frac{1}{x^{2}-w^{2}} \frac{1}{y^{2}-v^{2}}[g(x, y)-g(w, y)-g(x, v)+g(w, v)] & \simeq \frac{1}{2 x} \frac{\partial}{\partial x} \frac{1}{y^{2}-v^{2}}[g(x, y)-g(x, v)] \\
& \simeq \frac{1}{4 x y} \frac{\partial^{2}}{\partial x \partial y} g(x, y),
\end{aligned}
$$

com

$$
g(x, y)=\frac{f^{A}(x, y)}{4 x y\left[(x-y)^{2}-z^{2}\right]} .
$$

Substituindo as aproximações (5.61), (5.62), 5.63) e 5.64) de volta na equação 5.60 obtemos a aproximação de altas temperaturas para a amplitude estática como sendo

$$
\begin{aligned}
\Pi_{e}^{A} & \simeq\left\{\frac{1}{2 x} \frac{\partial}{\partial x}\left[\frac{f^{A}(x, x+z)}{4 z x\left[(x+z)^{2}-y^{2}\right]^{2}}\right]+[z \rightarrow-z]+\frac{1}{2 x} \frac{\partial}{\partial x}\left[\frac{f^{A}(x, x-z)}{4 z x\left[(x-z)^{2}-y^{2}\right]^{2}}\right]\right. \\
& +[x \rightarrow-x]+\frac{1}{2 y} \frac{\partial}{\partial y}\left[\frac{f^{A}(y+z, y)}{4 z y\left[(y+z)^{2}-x^{2}\right]^{2}}\right]+[y \rightarrow-y] \\
& \left.+\frac{1}{4 x y} \frac{\partial^{2}}{\partial x \partial y} \frac{f^{A}(x, y)}{4 x y\left[(x-y)^{2}-z^{2}\right]}+[y \rightarrow-y]\right\}+\{(x, z) \rightarrow(-x,-z)\}
\end{aligned}
$$

Vamos considerar agora o limite de momento externo nulo. Neste caso, a amplitude 5.57) se reduz à

$$
\Pi_{0}^{A}=\oint_{C} \frac{d p_{0}}{2 \pi i} \oint_{C} \frac{d q_{0}}{2 \pi i} \frac{f^{A}\left(p_{0}, q_{0}\right)}{\left(p_{0}^{2}-x^{2}\right)^{2}\left[\left(p_{0}-q_{0}\right)^{2}-z^{2}\right]\left(q_{0}^{2}-y^{2}\right)^{2}} .
$$

Usando o teorema dos resíduos, a integração em $p_{0}$ fornece

$$
\begin{aligned}
\Pi_{0}^{A} & =-\oint_{C} \frac{d q_{0}}{2 \pi i}\left\{\frac{f^{A}\left(q_{0}+z, q_{0}\right)}{2 z\left[\left(q_{0}+z\right)^{2}-x^{2}\right]^{2}\left(q_{0}^{2}-y^{2}\right)^{2}}+(z \rightarrow-z)\right. \\
& \left.+\lim _{p_{0} \rightarrow x} \frac{\partial}{\partial p_{0}} \frac{f^{A}\left(p_{0}, q_{0}\right)}{\left(p_{0}+x\right)^{2}\left[\left(p_{0}-q_{0}\right)^{2}-z^{2}\right]\left(q_{0}^{2}-y^{2}\right)^{2}}+(x \rightarrow-x)\right\} \\
& =-\oint_{C} \frac{d q_{0}}{2 \pi i}\left\{\left[\frac{f^{A}\left(q_{0}+z, q_{0}\right)}{2 z\left[\left(q_{0}+z\right)^{2}-x^{2}\right]^{2}\left(q_{0}^{2}-y^{2}\right)^{2}}+\frac{1}{2 x} \frac{\partial}{\partial x} \frac{f^{A}\left(x, q_{0}\right)}{2 x\left[\left(x-q_{0}\right)^{2}-z^{2}\right]\left(q_{0}^{2}-y^{2}\right)^{2}}\right]\right. \\
& +[(x, z) \rightarrow(-x,-z)]\} .
\end{aligned}
$$

Similarmente, a integração em $q_{0}$ produz

$$
\begin{aligned}
\Pi_{0}^{A} & =\left\{\lim _{q_{0} \rightarrow x-z} \frac{\partial}{\partial q_{0}} \frac{f^{A}\left(q_{0}+z, q_{0}\right)}{2 z\left(q_{0}+z+x\right)^{2}\left(q_{0}^{2}-y^{2}\right)^{2}}+(x \rightarrow-x)\right. \\
& +\lim _{q_{0} \rightarrow y} \frac{\partial}{\partial q_{0}} \frac{f^{A}\left(q_{0}+z, q_{0}\right)}{2 z\left[\left(q_{0}+z\right)^{2}-x^{2}\right]^{2}\left(q_{0}+y\right)^{2}}+(y \rightarrow-y) \\
& +\frac{1}{2 x} \frac{\partial}{\partial x} \lim _{q_{0} \rightarrow y} \frac{\partial}{\partial q_{0}} \frac{f^{A}\left(x, q_{0}\right)}{2 x\left[\left(x-q_{0}\right)^{2}-z^{2}\right]\left(q_{0}+y\right)^{2}}+(y \rightarrow-y) \\
& \left.+\frac{1}{2 x} \frac{\partial}{\partial x} \frac{f^{A}(x, x+z)}{4 x z\left[(x+z)^{2}-y^{2}\right]^{2}}+(z \rightarrow-z)\right\}+\{(x, z) \rightarrow(-x,-z)\}
\end{aligned}
$$




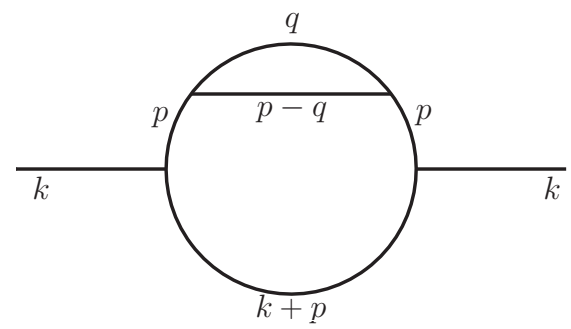

Figura 5.8: Segunda topologia para a auto-energia em ordem dois laços.

e avaliando os limites temos

$$
\begin{aligned}
\Pi_{0}^{A} & =\left\{\frac{1}{2 x} \frac{\partial}{\partial x} \frac{f^{A}(x, x-z)}{4 z x\left[(x-z)^{2}-y^{2}\right]^{2}}+(x \rightarrow-x)+\frac{1}{2 y} \frac{\partial}{\partial y} \frac{f^{A}(y+z, y)}{4 z y\left[(y+z)^{2}-x^{2}\right]^{2}}+(y \rightarrow-y)\right. \\
& \left.+\frac{1}{4 x y} \frac{\partial^{2}}{\partial x \partial y} \frac{f^{A}(x, y)}{4 x y\left[(x-y)^{2}-z^{2}\right]}+(y \rightarrow-y)+\frac{1}{2 x} \frac{\partial}{\partial x} \frac{f^{A}(x, x+z)}{4 x z\left[(x+z)^{2}-y^{2}\right]^{2}}+(z \rightarrow-z)\right\} \\
& +\{(x, z) \rightarrow(-x,-z)\}
\end{aligned}
$$

que é o mesmo do limite estático (5.66). Logo, quando os campos externos são estáticos, o limite de altas temperaturas da amplitude mostrada na figura 5.7 pode ser obtido supondo que todas as componentes do momento externo são nulas. É importante notar que obtivemos esta identidade mesmo antes de fazer a integral na parte espacial dos momentos $\vec{p}$ e $\vec{q}$.

\subsubsection{Segunda topologia}

Nesta seção iremos tratar a topologia mostrada na figura 5.8. No limite estático esta amplitude tem a forma

$$
\Pi_{e}^{B}=\oint_{C} \frac{d p_{0}}{2 \pi i} \oint_{C} \frac{d q_{0}}{2 \pi i} \frac{f^{B}\left(p_{0}, q_{0}\right)}{\left(p_{0}^{2}-x^{2}\right)^{2}\left(p_{0}^{2}-w^{2}\right)\left[\left(p_{0}-q_{0}\right)^{2}-z^{2}\right]\left(q_{0}^{2}-y^{2}\right)} .
$$

Neste caso introduzimos as quantidades $x=|\vec{p}|, y=|\vec{q}|, w=|\vec{p}+\vec{k}|$ e $z=|\vec{p}-\vec{q}|$. No limite de altas temperaturas o numerador $f^{B}$ possui as mesmas propriedades que $f^{A}$ na equação (5.58). A integração em $p_{0}$ e $q_{0}$ pode ser feita, como no caso anterior, usando o teorema dos resíduos, fornecendo 


$$
\begin{aligned}
\Pi_{e}^{B} & =\left\{\left[\frac{1}{2 x} \frac{\partial}{\partial x} \frac{f^{B}(x, x-z)}{4 x z\left(x^{2}-w^{2}\right)\left[(x-z)^{2}-y^{2}\right]}+(x \rightarrow-x)\right]+\left[\frac{f^{B}(w, w+z)}{4 z w\left(w^{2}-x^{2}\right)^{2}\left[(w+z)^{2}-y^{2}\right]}\right.\right. \\
& \left.+\frac{1}{2 x} \frac{\partial}{\partial x} \frac{f^{B}(x, x+z)}{4 x z\left(x^{2}-w^{2}\right)\left[(x+z)^{2}-y^{2}\right]}+(z \rightarrow-z)\right] \\
& +\left[\frac{f^{B}(w, w-z)}{4 z w\left(w^{2}-x^{2}\right)^{2}\left[(w-z)^{2}-y^{2}\right]}+(w \rightarrow-w)\right] \\
& +\left[\frac{f^{B}(y+z, y)}{4 z y\left[(y+z)^{2}-x^{2}\right]^{2}\left[(y+z)^{2}-w^{2}\right]}+\frac{f^{B}(w, y)}{4 w y\left(w^{2}-x^{2}\right)^{2}\left[(w-y)^{2}-z^{2}\right]}\right. \\
& \left.\left.+\frac{1}{2 x} \frac{\partial}{\partial x} \frac{f^{B}(x, y)}{4 y x\left(x^{2}-w^{2}\right)\left[(x-y)^{2}-z^{2}\right]}+(y \rightarrow-y)\right]\right\}+\{(x, w, z) \rightarrow(-x,-w,-z)\} .
\end{aligned}
$$

Na região dos HTL nos teremos $w=x+\epsilon$, com $\epsilon \ll x$. Expandindo $g(w)$ em série de Taylor em torno de $w=x$ até ordem segunda ordem, vemos que no limite de altas temperaturas teremos a aproximação

$$
\begin{aligned}
\frac{g(w)}{\left(w^{2}-x^{2}\right)^{2}}+\frac{1}{2 x} \frac{\partial}{\partial x} \frac{g(x)}{x^{2}-w^{2}} & \simeq \frac{g(x)}{\left(w^{2}-x^{2}\right)^{2}}+\frac{1}{(w+x)\left(w^{2}-x^{2}\right)} \frac{\partial}{\partial x} g(x) \\
& +\frac{1}{2(w+x)^{2}} \frac{\partial^{2}}{\partial x^{2}} g(x)-\frac{1}{\left(x^{2}-w^{2}\right)^{2}} g(x)+\frac{1}{2 x\left(x^{2}-w^{2}\right)} \frac{\partial}{\partial x} g(x) \\
& \simeq-\frac{1}{8 x^{3}} \frac{\partial}{\partial x} g(x)+\frac{1}{8 x^{2}} \frac{\partial^{2}}{\partial x^{2}} g(x)
\end{aligned}
$$

Substituindo a aproximação 5.73 na equação 5.72 temos

$$
\begin{aligned}
\Pi_{e}^{B} & \simeq\left\{\left[\left(\frac{1}{8 x^{2}} \frac{\partial^{2}}{\partial x^{2}}-\frac{1}{8 x^{3}} \frac{\partial}{\partial x}\right) \frac{f^{B}(x, x-z)}{4 x z\left[(x-z)^{2}-y^{2}\right]}+(x \rightarrow-x)\right]\right. \\
& +\left[\left(\frac{1}{8 x^{2}} \frac{\partial^{2}}{\partial x^{2}}-\frac{1}{8 x^{3}} \frac{\partial}{\partial x}\right) \frac{f^{B}(x, x+z)}{4 x z\left[(x+z)^{2}-y^{2}\right]}+(z \rightarrow-z)\right] \\
& \left.+\left[\left(\frac{1}{8 x^{2}} \frac{\partial^{2}}{\partial x^{2}}-\frac{1}{8 x^{3}} \frac{\partial}{\partial x}\right) \frac{f^{B}(x, y)}{4 y x\left[(x-y)^{2}-z^{2}\right]}+\frac{f^{B}(y+z, y)}{4 z y\left[(y+z)^{2}-x^{2}\right]^{3}}+(y \rightarrow-y)\right]\right\} \\
& +\{(x, z) \rightarrow(-x,-z)\} .
\end{aligned}
$$

É conveniente usar também a identidade

$$
\left(\frac{1}{8 x^{2}} \frac{\partial^{2}}{\partial x^{2}}-\frac{1}{8 x^{3}} \frac{\partial}{\partial x}\right) \frac{h(x)}{x}=\left(\frac{3}{8 x^{5}}-\frac{3}{8 x^{4}} \frac{\partial}{\partial x}+\frac{1}{8 x^{3}} \frac{\partial^{2}}{\partial x^{2}}\right) h(x),
$$


de modo que a amplitude estática tem a forma final

$$
\begin{aligned}
\Pi_{e}^{B} & \simeq\left\{\left[\left(\frac{3}{8 x^{5}}-\frac{3}{8 x^{4}} \frac{\partial}{\partial x}+\frac{1}{8 x^{3}} \frac{\partial^{2}}{\partial x^{2}}\right) \frac{f^{B}(x, x-z)}{4 z\left[(x-z)^{2}-y^{2}\right]}+(x \rightarrow-x)\right]\right. \\
& +\left[\left(\frac{3}{8 x^{5}}-\frac{3}{8 x^{4}} \frac{\partial}{\partial x}+\frac{1}{8 x^{3}} \frac{\partial^{2}}{\partial x^{2}}\right) \frac{f^{B}(x, x+z)}{4 z\left[(x+z)^{2}-y^{2}\right]}+(z \rightarrow-z)\right] \\
& +\left[\left(\frac{3}{8 x^{5}}-\frac{3}{8 x^{4}} \frac{\partial}{\partial x}+\frac{1}{8 x^{3}} \frac{\partial^{2}}{\partial x^{2}}\right) \frac{f^{B}(x, y)}{4 y\left[(x-y)^{2}-z^{2}\right]}+\frac{f^{B}(y+z, y)}{4 z y\left[(y+z)^{2}-x^{2}\right]^{3}}\right. \\
& +(y \rightarrow-y)]\}+\{(x, z) \rightarrow(-x,-z)\} .
\end{aligned}
$$

Vamos agora considerar o limite de momento externo nulo. Neste caso, a amplitude mostrada na figura 5.8 se reduz à

$$
\Pi_{0}^{B}=\oint_{C} \frac{d p_{0}}{2 \pi i} \oint_{C} \frac{d q_{0}}{2 \pi i} \frac{f^{B}\left(p_{0}, q_{0}\right)}{\left(p_{0}^{2}-x^{2}\right)^{3}\left[\left(p_{0}-q_{0}\right)^{2}-z^{2}\right]\left(q_{0}^{2}-y^{2}\right)} .
$$

Integrando em $p_{0}$ obtemos

$$
\begin{aligned}
\Pi_{0}^{B} & =-\oint_{C} \frac{d q_{0}}{2 \pi i}\left\{\frac{f^{B}\left(q_{0}+z, q_{0}\right)}{2 z\left[\left(q_{0}+z\right)^{2}-x^{2}\right]^{3}\left(q_{0}^{2}-y^{2}\right)}+(z \rightarrow-z)\right. \\
& \left.+\frac{1}{2} \lim _{p_{0} \rightarrow x} \frac{\partial^{2}}{\partial p_{0}^{2}} \frac{f^{B}\left(p_{0}, q_{0}\right)}{\left(p_{0}+x\right)^{3}\left[\left(p_{0}-q_{0}\right)^{2}-z^{2}\right]\left(q_{0}^{2}-y^{2}\right)}+(x \rightarrow-x)\right\}
\end{aligned}
$$

e calculando o limite, vemos que

$$
\begin{aligned}
\Pi_{0}^{B} & =\oint_{C} \frac{d q_{0}}{2 \pi i}\left\{\left[\left(\frac{3}{16 x^{5}}-\frac{3}{16 x^{4}} \frac{\partial}{\partial x}+\frac{1}{16 x^{3}} \frac{\partial^{2}}{\partial x^{2}}\right) \frac{f^{B}\left(x, q_{0}\right)}{\left[\left(x-q_{0}\right)^{2}-z^{2}\right]\left(q_{0}^{2}-y^{2}\right)}\right.\right. \\
& \left.\left.+\frac{f^{B}\left(q_{0}+z, q_{0}\right)}{2 z\left[\left(q_{0}+z\right)^{2}-x^{2}\right]^{3}\left(q_{0}^{2}-y^{2}\right)}\right]+[(x, z) \rightarrow(-x,-z)]\right\} .
\end{aligned}
$$


Finalmente, integrando $q_{0}$, teremos

$$
\begin{aligned}
\Pi_{0}^{B} & =\left\{\left[\frac{1}{2} \lim _{q_{0} \rightarrow x-z} \frac{\partial^{2}}{\partial q_{0}^{2}} \frac{f^{B}\left(q_{0}+z, q_{0}\right)}{2 z\left(q_{0}+z+x\right)^{3}\left(q_{0}^{2}-y^{2}\right)}+(x \rightarrow-x)\right]\right. \\
& +\left[\frac{f^{B}(y+z, y)}{4 z y\left[(y+z)^{2}-x^{2}\right]^{3}}+(y \rightarrow-y)\right] \\
& +\left[\left(\frac{3}{16 x^{5}}-\frac{3}{16 x^{4}} \frac{\partial}{\partial x}+\frac{1}{16 x^{3}} \frac{\partial^{2}}{\partial x^{2}}\right) \frac{f^{B}(x, y)}{2 y\left[(x-y)^{2}-z^{2}\right]}+(y \rightarrow-y)\right] \\
& \left.+\left[\left(\frac{3}{16 x^{5}}-\frac{3}{16 x^{4}} \frac{\partial}{\partial x}+\frac{1}{16 x^{3}} \frac{\partial^{2}}{\partial x^{2}}\right) \frac{f^{B}(x, x+z)}{2 z\left[(x+z)^{2}-y^{2}\right]}+(z \rightarrow-z)\right]\right\} \\
& +\{(x, z) \rightarrow(-x,-z)\} \\
& =\left\{\left[\left(\frac{3}{16 x^{5}}-\frac{3}{16 x^{4}} \frac{\partial}{\partial x}+\frac{1}{16 x^{3}} \frac{\partial^{2}}{\partial x^{2}}\right) \frac{f^{B}(x, x-z)}{2 z\left[(x-z)^{2}-y^{2}\right]}+(x \rightarrow-x)\right]\right. \\
& +\left[\frac{f^{B}(y+z, y)}{4 z y\left[(y+z)^{2}-x^{2}\right]^{3}}+(y \rightarrow-y)\right] \\
& +\left[\left(\frac{3}{16 x^{5}}-\frac{3}{16 x^{4}} \frac{\partial}{\partial x}+\frac{1}{16 x^{3}} \frac{\partial^{2}}{\partial x^{2}}\right) \frac{f^{B}(x, y)}{2 y\left[(x-y)^{2}-z^{2}\right]}+(y \rightarrow-y)\right] \\
& \left.+\left[\left(\frac{3}{16 x^{5}}-\frac{3}{16 x^{4}} \frac{\partial}{\partial x}+\frac{1}{16 x^{3}} \frac{\partial^{2}}{\partial x^{2}}\right) \frac{f^{B}(x, x+z)}{2 z\left[(x+z)^{2}-y^{2}\right]}+(z \rightarrow-z)\right]\right\} \\
& +\{(x, z) \rightarrow(-x,-z)\},
\end{aligned}
$$

que é idêntico ao resultado obtido na equação 5.76. Assim, quando os campos externos são estáticos, podemos computar o limite de altas temperaturas do diagrama mostrado na figura 5.8 supondo que todas as componentes do momento externo são nulas.

Portanto, os resultados desta seção, juntamente com os resultados do apêndice E, mostram que podemos computar as funções de Green estáticas no limite de altas temperaturas supondo que todas as componentes do momento são nulas, para todas as amplitudes 1PI em ordem um laço e para a auto-energia em ordem dois laços, para campos externos bosônicos. No contexto do método do campo de fundo introduzido na seção 5.1, vemos que podemos obter a ação efetiva de um campo bosônico externo considerando diagramas do vácuo para a pressão na presença de um campo constante, no limite de altas temperaturas. Nos dois próximos capítulos veremos na prática exemplos de como os resultados deste capítulo podem ser de fato aplicados para obter ações efetivas estáticas no contexto do método do campo de fundo. 


\section{QED em um campo de Maxwell externo e estático}

No capítulo 5 vimos que podemos obter o limite de altas temperaturas da ação efetiva estática de um campo bosônico calculando diagramas de vácuo para a pressão na presença de um campo independente do espaço-tempo. Com um exemplo concreto deste fato, iremos obter neste capítulo a ação efetiva para um campo de Maxwell interagindo com um plasma da QED até ordem de dois laços.

\subsection{QED em um campo eletromagnético externo}

\subsubsection{Ordem um laço}

A lagrangiana da QED no calibre de Feynman (veja a seção 4.1) possui a seguinte forma

$$
\mathcal{L}=-\frac{1}{4}\left(\partial_{\mu} \mathcal{A}_{\nu}-\partial_{\nu} \mathcal{A}_{\mu}\right)^{2}+i \bar{\psi} \gamma^{\mu}\left(\partial_{\mu}-i e \mathcal{A}_{\mu}\right) \psi-\frac{1}{2}\left(\partial^{\mu} \mathcal{A}_{\mu}\right)^{2}+\left(\partial_{\mu} \bar{C}\right)\left(\partial^{\mu} C\right)
$$

No método do campo de fundo vamos separar o campo de Maxwell $\mathcal{A}_{\mu}$ como

$$
\mathcal{A}_{\mu}=A_{\mu}+A_{\mu}^{C}
$$

onde $A_{\mu}^{C}$ representa um campo clássico e $A_{\mu}$ representa um campo quântico. Vamos supor que as fontes do campo clássicos $A_{\mu}^{C}$ são exteriores ao nosso problema, de modo que ele será tratado como um campo de fundo. Suponhamos também que o campo externo varia muito lentamente em relação a escala de tempo definida pelo inverso da temperatura, de modo que podemos aproximar $A_{\mu}^{C}$ como sendo um campo estático.

Como vimos no capítulo anterior, quando temos um campo externo estático, podemos computar o limite de altas temperaturas das funções de Green supondo que todas as componentes do momento externo são nulas. No espaço de configurações isto implica que 

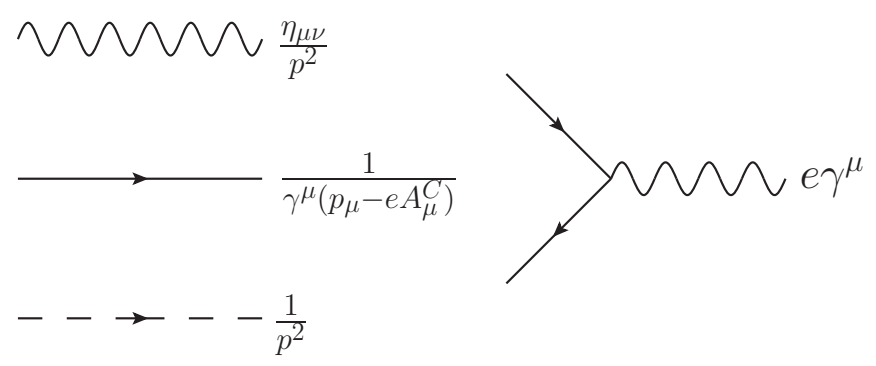

Figura 6.1: Regras de Feynman para a QED na presença de um campo de Maxwell externo.

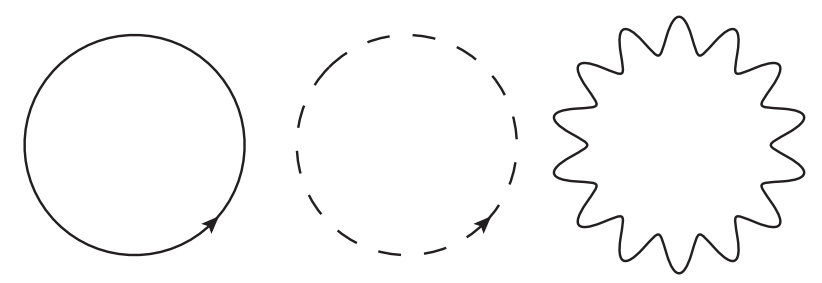

Figura 6.2: Contribuição em ordem de um laço para a pressão.

podemos considerar que o campo externo é independente do espaço tempo. Fazendo isto, a lagrangiana pode ser reescrita como

$$
\mathcal{L}=-\frac{1}{4}\left(\partial_{\mu} A_{\nu}-\partial_{\nu} A_{\mu}\right)^{2}+i \bar{\psi} \gamma^{\mu}\left[\left(\partial_{\mu}-i e A_{\mu}^{C}\right)-i e A_{\mu}\right] \psi-\frac{1}{2}\left(\partial^{\mu} A_{\mu}\right)^{2}+\left(\partial_{\mu} \bar{C}\right)\left(\partial^{\mu} C\right)
$$

e as regras de Feynman para esta teoria estão representadas na figura 6.1. Em ordem de um laço, os diagramas para a pressão estão representados na figura 6.2. Apenas o diagrama envolvendo o campo do férmion será uma função do campo de fundo $A_{\mu}^{C}$. Segundo a equação (5.15), podemos identificar a pressão com a lagrangiana efetiva. Portanto, a primeira contribuição para a Lagrangiana efetiva estática será

$$
\mathcal{L}_{\text {eff }}^{(1)}=\frac{1}{\beta V} \log \operatorname{Det} \beta i \gamma^{\mu}\left(p_{\mu}-e A_{\mu}^{C}\right)
$$

No formalismo do tempo imaginário nos temos

$$
\begin{aligned}
\mathcal{L}_{\text {eff }}^{(1)} & =\frac{2^{E(d / 2)-1}}{\beta V} \operatorname{Tr} \log \left[-\beta^{2}\left(p_{\mu}-e A_{\mu}^{C}\right)^{2}\right] \\
& =T 2^{E(d / 2)-1} \sum_{n} \int \frac{d^{d-1} p}{(2 \pi)^{d-1}} \log \left[-\beta^{2}\left(p_{\mu}-e A_{\mu}^{C}\right)^{2}\right],
\end{aligned}
$$

onde, $2^{E(d / 2)}$ é a dimensionalidade das matrizes de Dirac em um espaço-tempo $d$-dimensional, e $E(d / 2)$ é a parte inteira de $d / 2$, isto é, é o maior inteiro menor ou igual à $d / 2$. Fazendo uma mudança de variáveis na integral em $\vec{p}$ podemos eliminar a dependência em $\vec{A}$, ob- 
tendo

$$
\mathcal{L}_{\text {eff }}^{(1)}=\frac{T 2^{E(d / 2)-1}}{(2 \pi)^{d-1}} \sum_{n} \int d^{d-1} p \log \left\{\beta^{2}\left[-\left(i \omega_{n}-e A_{0}^{C}\right)^{2}+\vec{p}^{2}\right]\right\},
$$

onde $\omega_{n}=(2 n+1) \pi T$ é a frequência de Matsubara do férmion. Vale a pena notar que a lagrangiana efetiva estática depende apenas de $A_{0}^{C}$. A quantidade $e A_{0}^{C}$ tem um papel análogo ao de um potencial químico. Podemos reescrever a soma de Matsubara como

$$
\begin{aligned}
& \sum_{n} \log \left\{\beta^{2}\left[-\left(i \omega_{n}-e A_{0}^{C}\right)^{2}+\vec{p}^{2}\right]\right\} \\
& =\sum_{n} \frac{1}{2}\left\{\log \left\{\beta^{2}\left[\omega_{n}^{2}+2 i \omega_{n} e A_{0}^{C}-\left(e A_{0}^{C}\right)^{2}+\vec{p}^{2}\right]\right\}+\log \left\{\beta^{2}\left[\omega_{n}^{2}-2 i \omega_{n} e A_{0}^{C}-\left(e A_{0}^{C}\right)^{2}+\vec{p}^{2}\right]\right\}\right\} \\
& =\sum_{n} \frac{1}{2} \log \left\{\beta^{4}\left[\left(\omega_{n}^{2}+\vec{p}^{2}-\left(e A_{0}^{C}\right)^{2}\right)^{2}+4\left(e A_{0}^{C}\right)^{2} \omega_{n}^{2}\right]\right\} \\
& =\sum_{n} \frac{1}{2}\left\{\log \left\{\beta^{2}\left[\omega_{n}^{2}+\left(|\vec{p}|-e A_{0}^{C}\right)^{2}\right]\right\}+\log \left\{\beta^{2}\left[\omega_{n}^{2}+\left(|\vec{p}|+e A_{0}^{C}\right)^{2}\right]\right\}\right\}
\end{aligned}
$$

Portanto, temos que tratar as somas de Matsubara

$$
\begin{aligned}
S_{ \pm} & =\sum_{n} \log \left\{\beta^{2}\left[\omega_{n}^{2}+\left(|\vec{p}| \pm e A_{0}^{C}\right)^{2}\right]\right\} \\
& =\sum_{n}\left\{\int_{1}^{\beta^{2}\left(|\vec{p}| \pm e A_{0}^{C}\right)^{2}} d x^{2} \frac{1}{-[i(2 n+1) \pi]^{2}+x^{2}}+\log \left\{-[i(2 n+1) \pi]^{2}+1\right\}\right\} .
\end{aligned}
$$

Desprezando os termos independentes de $A_{0}^{C}$ temos

$$
S_{ \pm}=\int_{1}^{\beta^{2}\left(|\vec{p}| \pm e A_{0}^{C}\right)^{2}} d x^{2} \sum_{n} \frac{1}{-[i(2 n+1) \pi]^{2}+x^{2}}
$$

e agora a soma pode ser transformada em uma integral no plano complexo (veja apêndice C) fornecendo

$$
\begin{aligned}
S_{ \pm} & =\int_{1}^{\beta^{2}\left(|\vec{p}| \pm e A_{0}^{C}\right)^{2}} d x^{2}\left(\int_{-i \infty+\epsilon}^{i \infty+\epsilon} d z+\int_{-i \infty-\epsilon}^{+i \infty-\epsilon} d z\right) \frac{1}{4 \pi i} \tanh (z / 2) \frac{1}{-z^{2}+x} \\
& =\int_{1}^{\beta^{2}\left(|\vec{p}| \pm e A_{0}^{C}\right)^{2}} d x^{2} \frac{\tanh (x / 2)}{2 x} d x\left(1-\frac{2}{e^{x}+1}\right) \\
& =\int_{1}^{\beta\left(|\vec{p}| \pm e A_{0}^{C}\right)} d \log \left(1+e^{-\beta\left(|\vec{p}| \pm e A_{0}^{C}\right)}\right) \\
& =\beta\left(|\vec{p}| \pm e A_{0}^{C}\right)+2
\end{aligned}
$$


e assim teremos

$$
\mathcal{L}_{\text {eff }}^{(1)}=\frac{T 2^{E(d / 2)-1}}{(2 \pi)^{d-1}} \int d^{d-1} p\left\{\beta|\vec{p}|+\log \left(1+e^{-\beta\left(|\vec{p}|+e A_{0}^{C}\right)}\right)+\log \left(1+e^{-\beta\left(|\vec{p}|-e A_{0}^{C}\right)}\right)\right\},
$$

Desprezando a energia do ponto zero do oscilador que não depende do campo de fundo, temos

$$
\mathcal{L}_{e f f}^{(1)}=\frac{T^{d} 2^{E(d / 2)-1} \Omega_{d-1}}{(2 \pi)^{d-1}} \int_{0}^{\infty} d x x^{d-2}\left\{\log \left(1+e^{-\left(x+\beta e A_{0}^{C}\right)}\right)+\log \left(1+e^{-\left(x-\beta e A_{0}^{C}\right)}\right)\right\},
$$

onde $\Omega_{d-1}$ é o ângulo sólido. Neste ponto é conveniente fazer uma expansão no campo externo usando

$\log \left(1+e^{-(x+y)}\right)=\sum_{i=0}^{\infty} \frac{y^{i}}{i !} \lim _{y \rightarrow 0} \frac{d^{i}}{d y^{i}} \log \left(1+e^{-(x+y)}\right)=\log \left(1+e^{-x}\right)+\sum_{i=1}^{\infty} \frac{y^{i}}{i !} \frac{d^{i}}{d x^{i}} \log \left(1+e^{-x}\right)$

e portanto

$$
\log \left(1+e^{-(x+y)}\right)+\log \left(1+e^{-(x-y)}\right)=2 \log \left(1+e^{-x}\right)+2 \sum_{i=1}^{\infty} \frac{y^{2 i}}{(2 i) !} \frac{d^{2 i}}{d x^{2 i}} \log \left(1+e^{-x}\right) .
$$

Substituindo 6.14 em 6.12 e desprezando os termos que não dependem do campo externo obtemos

$$
\mathcal{L}_{\text {eff }}^{(1)}=\frac{2^{E(d / 2)} \Omega_{d-1}}{(2 \pi)^{d-1}} \sum_{i=1}^{\infty}\left[\frac{T^{d-2 i}\left(e A_{0}^{C}\right)^{2 i}}{(2 i) !} \int_{0}^{\infty} d x x^{d-2} \frac{d^{2 i}}{d x^{2 i}} \log \left(1+e^{-x}\right)\right] .
$$

A integral resultante está tabelada em termos das funções gama de Euler e zeta de Riemann, como segue

$$
\begin{aligned}
I & =\int_{0}^{\infty} d x x^{d-2} \frac{d^{2 i}}{d x^{2 i}} \log \left(1+e^{-x}\right)=-\int_{0}^{\infty} d x x^{d-2} \frac{d^{2 i-1}}{d x^{2 i-1}} \frac{1}{e^{x}-1} \\
& =-\int_{0}^{\infty} d x x^{d-2} \frac{d^{2 i-1}}{d x^{2 i-1}} \sum_{j=1}^{\infty}(-1)^{j+1} e^{-j x}=\int_{0}^{\infty} d x x^{d-2} \sum_{j=1}^{\infty}(-1)^{j+1} j^{2 i-1} e^{-j x} \\
& =\sum_{j=1}^{\infty} \frac{(-1)^{j+1}}{j^{d-2 i}} \int_{0}^{\infty} d x x^{d-2} e^{-x}=\left(1-2^{1+2 i-d}\right) \zeta(d-2 i) \Gamma(d-1),
\end{aligned}
$$




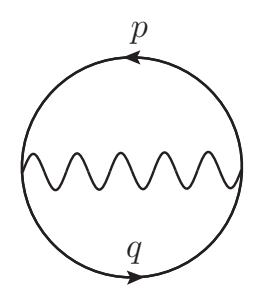

Figura 6.3: Contribuição de ordem dois laços para a pressão.

e assim substituindo em 6.15 obtemos

$$
\begin{aligned}
\mathcal{L}_{\text {eff }}^{(1)} & =\frac{2^{E(d / 2)} \Omega_{d-1} \Gamma(d-1)}{(2 \pi)^{d-1}} \sum_{i=1}^{\infty} \frac{T^{d-2 i}\left(e A_{0}^{C}\right)^{2 i}}{(2 i) !}\left(1-2^{1+2 i-d}\right) \zeta(d-2 i) \\
& =\frac{\Gamma(d-1)}{2^{d-E(d / 2)-2} \Gamma\left(\frac{d-1}{2}\right) \pi^{(d-1) / 2}} \sum_{i=1}^{\infty} \frac{T^{d-2 i}\left(e A_{0}^{C}\right)^{2 i}}{(2 i) !}\left(1-2^{1+2 i-d}\right) \zeta(d-2 i) .
\end{aligned}
$$

Em particular para a função de dois pontos teremos

$$
\left.\frac{\delta^{2}}{\delta A_{\mu}^{C} \delta A_{\nu}^{C}} \mathcal{L}_{e f f}^{(1)}\right|_{A^{C}=0}=u^{\mu} u^{\nu} \frac{e^{2} T^{d-2} \Gamma(d-1)\left(1-2^{3-d}\right) \zeta(d-2)}{2^{d-E(d / 2)-2} \Gamma\left(\frac{d-1}{2}\right) \pi^{(d-1) / 2}}=2 m^{2} u^{\mu} u^{\nu},
$$

onde $m^{2}$ é a massa térmica do fóton 4.70). Portanto, comparando com 4.111, verificamos que a lagrangiana efetiva estática (6.17) de fato gera corretamente a auto-energia estática, isto é

$$
\left.\frac{\delta^{2}}{\delta A_{\mu}^{C} \delta A_{\nu}^{C}} \mathcal{L}_{e f f}^{(1)}\right|_{A^{C}=0}=-\Pi^{\mu \nu}\left(k_{0}=0\right)
$$

A identidade $\zeta(-2 j)=0$ para $j=1,2, \ldots$ implica que para $d$ par apenas potências não negativas da temperatura $T$ contribuem para (6.17). Entretanto, para $d$ ímpar os termos com potências negativas da temperaturas na equação 6.17) devem ser suprimidos do ponto de vista físico, pois estamos no limite de altas temperaturas.

\subsubsection{Ordem de dois laços}

Em ordem de dois laços, o diagrama que contribui para a pressão está representado na figura 6.3. Logo, no formalismo do tempo imaginário temos a seguinte expressão analítica associada para a lagrangiana efetiva estática

$$
\mathcal{L}_{\text {eff }}^{(2)}=-\frac{e^{2} T^{2}}{2} \sum_{n_{p} n_{q}} \int \frac{d^{d-1} p}{(2 \pi)^{d-1}} \frac{d^{d-1} q}{(2 \pi)^{d-1}} \operatorname{tr}\left(\gamma^{\mu} \frac{1}{\mathbb{\pi}_{p}} \gamma_{\mu} \frac{1}{\pi_{q}}\right) \frac{1}{(p-q)^{2}},
$$


onde introduzimos a notação $\pi_{p}^{\mu}=p^{\mu}-e A^{\mu}$ e $\pi_{q}^{\mu}=q^{\mu}-e A^{\mu}$. Calculando o traço obtemos

$$
\mathcal{L}_{\text {eff }}^{(2)}=e^{2} T^{2} 2^{E(d / 2)-1}(d-2) \sum_{n_{p} n_{q}} \int \frac{d^{d-1} p}{(2 \pi)^{d-1}} \frac{d^{d-1} q}{(2 \pi)^{d-1}} \frac{\pi_{p} \cdot \pi_{q}}{\pi_{p}^{2} \pi_{q}^{2}(p-q)^{2}} .
$$

e usando a identidade algébrica

$$
\begin{aligned}
\pi_{p} \cdot \pi_{q} & =-\frac{1}{2}\left[\left(\pi_{p}-\pi_{q}\right)^{2}-\pi_{p}^{2}-\pi_{q}^{2}\right] \\
& =-\frac{1}{2}\left[(p-q)^{2}-\pi_{p}^{2}-\pi_{q}^{2}\right]
\end{aligned}
$$

temos

$$
\begin{aligned}
\mathcal{L}_{\text {eff }}^{(2)} & =e^{2} T^{2} 2^{E(d / 2)-2}(2-d) \sum_{n_{p} n_{q}} \int \frac{d^{d-1} p}{(2 \pi)^{d-1}} \frac{d^{d-1} q}{(2 \pi)^{d-1}} \\
& \times\left[\frac{1}{\pi_{p}^{2} \pi_{q}^{2}}-\frac{1}{\pi_{q}^{2}(p-q)^{2}}-\frac{1}{\pi_{p}^{2}(p-q)^{2}}\right]
\end{aligned}
$$

Fazendo algumas mudanças de variáveis na integração espacial em $\vec{p}$ e $\vec{q}$ podemos eliminar a dependência em $\vec{A}$, obtendo

$$
\begin{aligned}
\mathcal{L}_{\text {eff }}^{(2)} & =e^{2} T^{2} 2^{E(d / 2)-2}(2-d)\left\{\left[\sum_{\omega_{n}^{F}} \int \frac{d^{d-1} p}{(2 \pi)^{d-1}} \frac{1}{-\left(i \omega_{n}^{F}-e A_{0}^{C}\right)^{2}+\vec{p}^{2}}\right]^{2}\right. \\
& \left.-2\left[\sum_{\omega_{n}^{F}} \int \frac{d^{d-1} p}{(2 \pi)^{d-1}} \frac{1}{-\left(i \omega_{n}^{F}-e A_{0}^{C}\right)^{2}+\vec{p}^{2}}\right]\left[\sum_{\omega_{n}^{B}} \int \frac{d^{d-1} k}{(2 \pi)^{d-1}} \frac{1}{-\left(i \omega_{n}^{B}\right)^{2}+\vec{k}^{2}}\right]\right\} . \\
& =e^{2} 2^{E(d / 2)-2}(2-d)\left[I_{F}\left(I_{F}-2 I_{B}\right)\right] .
\end{aligned}
$$

Fazendo soma de Matsubara do termo bosônico temos

$$
\begin{aligned}
I_{B} & =T \sum_{\omega_{n}^{B}} \int \frac{d^{d-1} k}{(2 \pi)^{d-1}} \frac{1}{-\left(i \omega_{n}^{B}\right)^{2}+\vec{k}^{2}} \\
& =\int \frac{d^{d-1} k}{(2 \pi)^{d-1}} \frac{1}{4 \pi i}\left[\int_{-i \infty+\epsilon}^{i \infty+\epsilon} d k_{0}+\int_{i \infty-\epsilon}^{-i \infty-\epsilon} d k_{0}\right] \frac{\operatorname{coth}\left(\beta p_{0} / 2\right)}{-k_{0}^{2}+\vec{k}^{2}} \\
& =\int \frac{d^{d-1} k}{(2 \pi)^{d-1}} \frac{1+2 N_{B}(|\vec{k}|)}{2|\vec{k}|}
\end{aligned}
$$


e para os termos fermiônicos obtemos

$$
\begin{aligned}
I_{F} & =T \sum_{\omega_{n}^{F}} \int \frac{d^{d-1} p}{(2 \pi)^{d-1}} \frac{1}{-\left(i \omega_{n}^{F}-e A_{0}^{C}\right)^{2}+\vec{p}^{2}} \\
& =\int \frac{d^{d-1} p}{(2 \pi)^{d-1}} \frac{1}{4 \pi i}\left[\int_{-i \infty+\epsilon}^{i \infty+\epsilon} d p_{0}+\int_{i \infty-\epsilon}^{-i \infty-\epsilon} d p_{0}\right] \frac{\tanh \left(\beta p_{0} / 2\right)}{-\left(p_{0}-e A_{0}^{C}\right)^{2}+\vec{p}^{2}} \\
& =\int \frac{d^{d-1} p}{(2 \pi)^{d-1}} \frac{1}{2}\left[\frac{\tanh \left(\beta\left(|\vec{p}|+e A_{0}^{C}\right) / 2\right)}{2|\vec{p}|}+\frac{\tanh \left(\beta\left(|\vec{p}|-e A_{0}^{C}\right) / 2\right)}{2|\vec{p}|}\right] \\
& =\int \frac{d^{d-1} p}{(2 \pi)^{d-1}} \frac{1-N_{F}\left(|\vec{p}|+e A_{0}\right)-N_{F}\left(|\vec{p}|-e A_{0}\right)}{2|\vec{p}|}
\end{aligned}
$$

Os termos proporcionais a unidade em 6.25 e 6.26), dão origem a termos que são independentes e lineares nas distribuição térmica em (6.24). O termo que é independente da distribuição térmica é justamente a contribuição de temperatura zero para a energia de vácuo e pode ser desprezado. Os termos lineares nas distribuições térmicas serão subdominantes no limite de altas temperaturas [KG06]. Assim podemos ignorar os termos proporcionais a unidade em 6.25) e 6.26). Para o termo bosônico, a parte puramente térmica fornece

$$
\begin{aligned}
I_{B} & =\int \frac{d^{d-1} k}{(2 \pi)^{d-1}} \frac{N_{B}(|\vec{k}|)}{|\vec{k}|} \\
& =\frac{T^{d-2} \Omega_{d-1}}{(2 \pi)^{d-1}} \int_{0}^{\infty} d x x^{d-3} \frac{1}{e^{x}-1} \\
& =\frac{T^{d-2} \Omega_{d-1} \Gamma(d-2) \zeta(d-2)}{(2 \pi)^{d-1}}
\end{aligned}
$$

e para o termo fermiônico teremos

$$
\begin{aligned}
I_{F} & =-\int \frac{d^{d-1} p}{(2 \pi)^{d-1}} \frac{N_{F}\left(|\vec{p}|+e A_{0}^{C}\right)+N_{F}\left(|\vec{p}|-e A_{0}^{C}\right)}{2|\vec{p}|} \\
& =-\frac{T^{d-2} \Omega_{d-1}}{2(2 \pi)^{d-1}} \int_{0}^{\infty} d x x^{d-3}\left(\frac{1}{e^{x+\beta e A_{0}^{C}}+1}+\frac{1}{e^{x-\beta e A_{0}^{C}}+1}\right)
\end{aligned}
$$

Para avaliar a integral resultante, faremos uma expansão em série de Taylor no termo entre parênteses na forma

$$
I_{F}=-\frac{T^{d-2} \Omega_{d-1}}{2(2 \pi)^{d-1}} \int_{0}^{\infty} d x x^{d-3} 2 \sum_{n=0}^{\infty} \frac{\left(\beta e A_{0}^{C}\right)^{2 n}}{(2 n) !} \frac{d^{2 n}}{d x^{2 n}} \frac{1}{e^{x}+1} .
$$


Agora podemos fazer a integral em termos de funções especiais

$$
\begin{aligned}
J & =\int_{0}^{\infty} d x x^{d-3} \frac{d^{2 n}}{d x^{2 n}} \frac{1}{e^{x}+1} \\
& =\int_{0}^{\infty} d x x^{d-3} \frac{d^{2 n}}{d x^{2 n}} \sum_{j=1}^{\infty}(-1)^{j+1} e^{-j x} \\
& =\sum_{j=1}^{\infty} \frac{(-1)^{j+1}}{j^{d-2 n-2}} \int_{0}^{\infty} d x x^{d-3} e^{-x} \\
& =\left(1-2^{2 n-d+3}\right) \zeta(d-2 n-2) \Gamma(d-2),
\end{aligned}
$$

e assim teremos

$$
I_{F}=-\frac{\Gamma(d-2) \Omega_{d-1}}{(2 \pi)^{d-1}} \sum_{n=0}^{\infty} T^{d-2 n-2} \frac{\left(e A_{0}^{C}\right)^{2 n}}{(2 n) !}\left(1-2^{2 n-d+3}\right) \zeta(d-2 n-2) .
$$

Substituindo 6.31) e 6.27) em 6.24) obtemos

$$
\begin{aligned}
\mathcal{L}_{\text {eff }}^{(2)} & =\frac{e^{2} \Omega_{d-1}^{2} 2^{E(d / 2)-2}(2-d)[\Gamma(d-2)]^{2}}{(2 \pi)^{2 d-2}}\left\{\sum_{n=0}^{\infty} T^{d-2 n-2} \frac{\left(e A_{0}^{C}\right)^{2 n}}{(2 n) !}\left(1-2^{2 n-d+3}\right) \zeta(d-2 n-2)\right\} \\
& \times\left\{2 T^{d-2} \zeta(d-2)+\sum_{n=0}^{\infty} T^{d-2 n-2} \frac{\left(e A_{0}^{C}\right)^{2 n}}{(2 n) !}\left(1-2^{2 n-d+3}\right) \zeta(d-2 n-2)\right\} \\
& =\frac{e^{2}[\Gamma(d-2)]^{2}(2-d)}{2^{2 d-E(d / 2)-2} \pi^{d-1}\left[\Gamma\left(\frac{d-1}{2}\right)\right]^{2}}\left\{\sum_{n=0}^{\infty} T^{d-2 n-2} \frac{\left(e A_{0}^{C}\right)^{2 n}}{(2 n) !}\left(1-2^{2 n-d+3}\right) \zeta(d-2 n-2)\right\} \\
& \times\left\{2 T^{d-2} \zeta(d-2)+\sum_{n=0}^{\infty} T^{d-2 n-2} \frac{\left(e A_{0}^{C}\right)^{2 n}}{(2 n) !}\left(1-2^{2 n-d+3}\right) \zeta(d-2 n-2)\right\} .
\end{aligned}
$$

Para $d=4$ a série possui apenas dois termos pois $\zeta(-2 j)=0$ para $j=1,2, \ldots$, e assim teremos

$$
\begin{aligned}
\sum_{n=0}^{\infty} T^{d-2 n-2} \frac{\left(e A_{0}^{C}\right)^{2 n}}{(2 n) !}\left(1-2^{2 n-d+3}\right) \zeta(d-2 n-2) & =T^{2}\left(1-2^{-1}\right) \zeta(2)+\left(e A_{0}\right)^{2} \frac{1-2}{2} \zeta(0) \\
& =\frac{T^{2} \pi^{2}}{12}+\frac{\left(e A_{0}\right)^{2}}{4} .
\end{aligned}
$$

Portanto para $d=4$ temos

$$
\begin{aligned}
\mathcal{L}_{\text {eff }}^{(2)} & =-\frac{2 e^{2}[\Gamma(2)]^{2}}{2^{4} \pi^{3}\left[\Gamma\left(\frac{3}{2}\right)\right]^{2}}\left[\frac{T^{2} \pi^{2}}{12}+\frac{\left(e A_{0}\right)^{2}}{4}\right]\left[\frac{2 T^{2} \pi^{2}}{6}+\frac{T^{2} \pi^{2}}{12}+\frac{\left(e A_{0}\right)^{2}}{4}\right] \\
& =-\frac{e^{2}}{288 \pi^{4}}\left(T^{2} \pi^{2}+3\left(e A_{0}\right)^{2}\right)\left(5 T^{2} \pi^{2}+3\left(e A_{0}\right)^{2}\right) \\
& =-\frac{e^{2}}{288 \pi^{4}}\left(5 T^{4} \pi^{4}+18 T^{2}\left(e A_{0}\right)^{2} \pi^{2}+9\left(e A_{0}\right)^{4} \pi^{4}\right) .
\end{aligned}
$$


Logo, a auto-energia estática em ordem dois laços possui então o seguinte limite de altas temperaturas

$$
\begin{aligned}
\Pi_{2}^{\mu \nu}\left(k_{0}=0\right) & =\left.\frac{\delta^{2} \mathcal{L}_{\text {eff }}^{(2)}}{\delta A_{\mu}^{C} \delta A_{\nu}^{C}}\right|_{A^{C}=0} \\
& =-\frac{e^{4} T^{2 d-6}[\Gamma(d-2)]^{2}(2-d)\left(1-2^{5-d}\right) \zeta(d-4) \zeta(d-2)\left(4-2^{4-d}\right)}{2^{2 d-E(d / 2)-2} \pi^{d-1}\left[\Gamma\left(\frac{d-1}{2}\right)\right]^{2}} u^{\mu} u^{\nu}
\end{aligned}
$$

Usando a expressão 6.17 vemos que até ordem de dois laços a auto-energia estática para $d=4$ tem a forma

$$
\Pi^{\mu \nu}\left(k_{0}=0\right)=-\left[\frac{e^{2} T^{2}}{3}-\frac{T^{2} e^{4}}{8 \pi^{2}}\right] u^{\mu} u^{\nu}, \quad(d=4),
$$

e a massa térmica do fóton tem a forma

$$
m^{2}=\frac{e^{2} T^{2}}{6}-\frac{T^{2} e^{4}}{16 \pi^{2}}, \quad(d=4)
$$




\section{Campos térmicos na presença de um campo gravitacional}

Neste capítulo, estudaremos campos térmicos na presença de um campo gravitacional. No contexto do método do campo de fundo, vamos considerar um campo gravitacional estático. As fontes do campo gravitacional são campos de matéria exteriores ao nosso sistema térmico e no sistema de repouso do banho térmico a métrica não depende explicitamente do tempo. Logo, podemos utilizar os métodos introduzidos no capítulo 5 para obter a ação efetiva estática de teoria. Isto será feito em dois contextos distintos. Primeiro, no caso do campo escalar livre a ação efetiva será obtida até ordem de um laço [BS12a]. Depois, para um plasma da QED obteremos a ação efetiva ate ordem dois laços.

\subsection{Campo escalar livre}

\subsubsection{Método perturbativo}

No apêndice G vimos como escrever as derivadas covariantes de campos com spin arbitrários em espaços curvos, a partir de sua respectiva representação do grupo de Lorentz no espaço localmente euclideano. Em particular, no caso do campo escalar por transformação de Lorentz, não exite a necessidade de introduzir tais derivadas covariante. Deste modo, para um campo escalar livre sem massa, minimamente acoplado com o campo gravitacional, a ação possui então a forma simples

$$
S=\int d^{d} x \sqrt{-g} \frac{1}{2} g^{\mu \nu} \partial_{\mu} \phi \partial_{\nu} \phi
$$

onde denotamos $\sqrt{-g}=\sqrt{|\operatorname{det} g|}=\sqrt{(-1)^{d-1} \operatorname{det} g}$. Como vimos no capítulo 5 , podemos obter o limite de altas temperaturas da ação efetiva de um campo externo e estático considerando configurações de campo independentes do espaço-tempo. Portanto, como estamos restritos ao caso de uma métrica que não depende do tempo no sistema de repouso 
do banho térmico, iremos supor que a métrica é constante.

A função de partição neste caso será então

$$
Z=\int \mathcal{D} \phi \exp \left\{\int_{0}^{\beta} d^{d} x \sqrt{-g} \frac{1}{2} g^{\mu \nu} \partial_{\mu} \phi \partial_{\nu} \phi\right\},
$$

com a integração funcional se dando sobre modos periódicos do campo. Portanto usando os resultados da seção 5.1, temos que a lagrangiana efetiva pode ser escrita como o determinante funcional

$$
\mathcal{L}=\frac{T}{V} \log \left[\operatorname{Det}\left(-\beta^{2} p_{\mu} \sqrt{-g} g^{\mu \nu} p_{\nu}\right)\right]^{-1 / 2}
$$

Considerando um método perturbativo, vamos escrever o campo de gráviton, denotado por $\tilde{h}^{\mu \nu}$, como em [Gol58], na forma

$$
\sqrt{-g} g^{\mu \nu} \equiv \tilde{g}^{\mu \nu}=\eta^{\mu \nu}+\tilde{h}^{\mu \nu}
$$

onde teremos o campo do gráviton $\tilde{h}^{\mu \nu}$, em uma métrica de fundo de Minkowski $\eta^{\mu \nu}$. Substituindo a expansão (7.4) na equação (7.3), obtemos

$$
\mathcal{L}=\frac{T}{V} \log \left[\operatorname{Det}\left(-\beta^{2} p^{2}\right) \operatorname{Det}\left(1+\frac{1}{p^{2}} p_{\mu} \tilde{h}^{\mu \nu} p_{\nu}\right)\right]^{-1 / 2},
$$

com a notação $p^{2}=\eta^{\mu \nu} p_{\mu} p_{\nu}$. Como estamos considerando uma métrica constante, o campo do gráviton comuta com a derivada, de modo que teremos

$$
\begin{aligned}
\mathcal{L}^{\text {stat. }} & =T \log \left[\operatorname{Det}\left(-\beta^{2} p^{2}\right) \operatorname{Det}\left(1+\tilde{h}^{\mu \nu} \frac{p_{\mu} p_{\nu}}{p^{2}}\right)\right]^{-1 / 2} \\
& =\mathcal{L}^{(0)}-\frac{1}{2} \frac{T}{V} \log \operatorname{Det}\left(1+\tilde{h}^{\mu \nu} \frac{p_{\mu} p_{\nu}}{p^{2}}\right)
\end{aligned}
$$

onde $\mathcal{L}^{(0)}$ é a pressão do campo escalar livre [KG06, Bel96], que foi obtida no capítulo 3 como

$$
\mathcal{L}^{(0)}=\frac{\Gamma[d] \zeta(d)}{2^{d-2} \pi^{(d-1) / 2} \Gamma\left(\frac{d-1}{2}\right)(d-1)} T^{d}
$$

Para a parte dependente do campo gravitacional teremos

$$
\mathcal{L}^{I}=-\frac{1}{2} \frac{T}{V} \log \operatorname{Det}\left(1+\tilde{h}^{\mu \nu} \frac{p_{\mu} p_{\nu}}{p^{2}}\right) .
$$


Usando a relação $\log \operatorname{Det} A=\operatorname{Tr} \log A$, no formalismo do tempo imaginário obtemos

$$
\mathcal{L}^{I}=-\frac{T}{2} \sum_{n=-\infty}^{\infty} \int \frac{d^{d-1} p}{(2 \pi)^{d-1}} \log \left(1+\tilde{h}^{\mu \nu} \frac{p_{\mu} p_{\nu}}{p^{2}}\right)
$$

onde $p^{2}=\eta^{\mu \nu} p_{\mu} p_{\nu}=p_{0}^{2}-\vec{p}^{2}=-(2 \pi n T)^{2}-\vec{p}^{2}$.

Fazendo uma expansão do logaritmo na forma $\log (1+x)=x-x^{2} / 2+x^{3} / 3+\cdots$ em 7.9 obtemos uma expansão em potências de $\tilde{h}^{\mu \nu}$ na forma

$$
\begin{aligned}
\mathcal{L}^{I} & =-\frac{T}{2} \sum_{n=-\infty}^{\infty} \int \frac{d^{d-1} p}{(2 \pi)^{d-1}}\left(\tilde{h}^{\mu \nu} \frac{p_{\mu} p_{\nu}}{p^{2}}-\frac{\tilde{h}^{\mu \nu} \tilde{h}^{\alpha \beta}}{2} \frac{p_{\mu} p_{\nu} p_{\alpha} p_{\beta}}{p^{4}}+\cdots\right) \\
& =\tilde{h}^{\mu \nu} I_{\mu \nu}+\frac{1}{2} \tilde{h}^{\mu \nu} \tilde{h}^{\alpha \beta} I_{\mu \nu \alpha \beta}+\cdots,
\end{aligned}
$$

onde

$$
I_{\mu \nu}=-\frac{T}{2} \sum_{n=-\infty}^{\infty} \int \frac{d^{d-1} p}{(2 \pi)^{d-1}} \frac{p_{\mu} p_{\nu}}{p^{2}}
$$

e

$$
I_{\mu \nu \alpha \beta}=\frac{T}{2} \sum_{n=-\infty}^{\infty} \int \frac{d^{d-1} p}{(2 \pi)^{d-1}} \frac{p_{\mu} p_{\nu} p_{\alpha} p_{\beta}}{p^{4}} .
$$

Podemos interpretar cada termo na equação 7.10 como a amplitude 1PI estática de $n$ pontos ( que pode ser identificada com a respectiva amplitude de momento nulo) em ordem de um laço, contraída com $n$ campos $\tilde{h}^{\mu \nu}$. O cálculo dos dois primeiros termos em 7.10 está apresentado no apêndice $\mathrm{H}$.

Combinando as equações (7.6), (7.10), (H.2), (H.6), (H.8) e (H.14), a lagrangiana efetiva pode ser escrita como segue

$$
\mathcal{L}^{\text {stat. }}=\mathcal{L}^{(0)}+\tilde{\Gamma}_{\mu \nu} \tilde{h}^{\mu \nu}+\frac{1}{2} \tilde{\Pi}_{\mu \nu \alpha \beta} \tilde{h}^{\mu \nu} \tilde{h}^{\alpha \beta}+\cdots
$$

onde

$$
\tilde{\Gamma}_{\mu \nu}=\frac{\mathcal{L}^{(0)}}{2}\left(d u_{\mu} u_{\nu}-\eta_{\mu \nu}\right)
$$

e

$$
\tilde{\Pi}_{\mu \nu \alpha \beta}=\mathcal{L}^{(0)}\left[\tilde{\Gamma}_{\mu \nu} \tilde{\Gamma}_{\alpha \beta}+\tilde{\Gamma}_{\mu \alpha} \tilde{\Gamma}_{\nu \beta}+\tilde{\Gamma}_{\mu \beta} \tilde{\Gamma}_{\nu \alpha}-\frac{d(d-1)}{2} u_{\mu} u_{\nu} u_{\alpha} u_{\beta}\right]
$$

Ambos resultados da função de um ponto (7.14 e da função de dois pontos (7.15) são exatamente os mesmos obtidos das amplitudes estáticas no limite de altas temperaturas 


\section{BF98.}

Podemos verificar que existem identidades de Weyl relacionando $\tilde{\Pi}_{\mu \nu \alpha \beta} \operatorname{com} \tilde{\Gamma}_{\mu \nu}$, na forma

$$
\begin{aligned}
\eta^{\mu \nu} \tilde{\Gamma}_{\mu \nu} & =0, \\
\eta^{\mu \nu} \tilde{\Pi}_{\mu \nu \alpha \beta} & =-\tilde{\Gamma}_{\mu \nu} .
\end{aligned}
$$

Estas identidades são uma consequência da invariância conforme da lagrangiana efetiva (7.3) pela transformação $\sqrt{-g} g^{\mu \nu} \rightarrow(1+\epsilon) \sqrt{-g} g^{\mu \nu}$, que é equivalente à $\tilde{h}^{\mu \nu} \rightarrow$ $\tilde{h}^{\mu \nu}+\epsilon \tilde{h}^{\mu \nu}+\epsilon \eta^{\mu \nu}$, com $\epsilon$ infinitesimal. Mesmo no caso não estático, é conhecido que as identidades de Weyl são satisfeitas pelo limite de altas temperaturas das amplitudes térmicas [BF93a]. Esta é uma informação importante que vincula a lagrangiana efetiva a ser invariante conforme mesmo fora do limite estático, embora sua forma explícita ainda não seja conhecida.

Podemos verificar que $\mathcal{L}^{\text {stat }}$ é independente da representação do campo do gráviton. Como um exemplo, alternativamente podemos definir o campo do gráviton como

$$
\bar{h}^{\mu \nu}=g^{\mu \nu}-\eta^{\mu \nu}
$$

A relação entre as representações $\tilde{h}^{\mu \nu}$ e $\bar{h}^{\mu \nu}$ pode ser escrita como

$$
\tilde{h}^{\mu \nu}=\bar{h}^{\mu \nu}-\eta^{\mu \nu}\left(\frac{\bar{h}}{2}-\frac{1}{4} \bar{h}_{\alpha \beta} \bar{h}^{\beta \alpha}-\frac{\bar{h}^{2}}{8}\right)-\frac{\bar{h}}{2} \bar{h}^{\mu \nu}+\mathcal{O}\left(\bar{h}^{3}\right),
$$

onde os índices são abaixados e levantados pela métrica de Minkowski. Inserindo a equação 7.18 em 7.13 e usando as identidades de Weyl 7.16 podemos ver que $\mathcal{L}^{\text {stat. }}$ possui a mesma forma quando o campo do gráviton é definido como $\bar{h}^{\mu \nu}$. Esta independência na parametrização do campo do gráviton é de fato esperada para uma quantidade física, tal como a lagrangiana efetiva, mesmo quando a transformação no campo não é induzida por um simples reescalonamento da métrica, como no exemplo anterior. Em geral, o campo e as amplitudes se transformam de uma maneira a manter a invariância da lagrangiana efetiva [BF93a].

De fato, transformando o campo do gráviton para $h_{\mu \nu}=g_{\mu \nu}-\eta_{\mu \nu}$, obtemos

$$
\mathcal{L}^{\text {stat. }}=\mathcal{L}^{(0)}+\Gamma^{\mu \nu} h_{\mu \nu}+\frac{1}{2} \Pi^{\mu \nu \alpha \beta} h_{\mu \nu} h_{\alpha \beta}+\cdots
$$


onde

$$
\Gamma^{\mu \nu}=-\frac{\mathcal{L}^{(0)}}{2}\left(d u^{\mu} u^{\nu}-\eta^{\mu \nu}\right)
$$

e

$$
\Pi^{\mu \nu \alpha \beta}=\mathcal{L}^{(0)}\left[\Gamma^{\mu \nu} \Gamma^{\alpha \beta}-\frac{1}{4}\left(\eta^{\mu \alpha} \eta^{\nu \beta}+\eta^{\mu \beta} \eta^{\nu \alpha}\right)+\frac{d}{2} u^{\mu} u^{\nu} u^{\alpha} u^{\beta}\right]
$$

que estão de acordo com as amplitudes estáticas obtidas em [Reb91]. As amplitudes $\Gamma^{\mu \nu}$ e $\Pi^{\mu \nu \alpha \beta}$ também satisfazem as identidades de Weyl $(7.16)$. Vale a pena ressaltar que enquanto o resultado (7.21) uma simetria bosônica $(\mu \nu) \leftrightarrow(\alpha \beta)$ associada com a métrica (além das simetrias $\mu \leftrightarrow \nu$ e $\alpha \leftrightarrow \beta$ ), a equação 7.15 possui uma simetria tensorial mais ampla, em todos os índices, que pode ser antecipada da equação 7.12 .

\subsubsection{Métrica estática geral}

Agora vamos considerar um cenário físico mais geral em que a métrica de fundo continua sendo estática, mas não é necessariamente próxima à métrica de Minkowski. Usando novamente a relação $\log \operatorname{Det} A=\operatorname{Tr} \log A$ no contexto do formalismo do tempo imaginário, a equação (7.3) fornece

$$
\mathcal{L}=-\frac{T}{2} \sum_{n} \int \frac{d^{d-1} p}{(2 \pi)^{d-1}} \log \left(-\beta^{2} p_{\mu} \tilde{g}^{\mu \nu} p_{\nu}\right)
$$

Usando que no limite de altas temperaturas a métrica estática pode ser aproximada por uma métrica constante, a lagrangiana efetiva pode ser escrita como segue

$$
\mathcal{L}^{\text {stat. }}=-\frac{T}{2} \sum_{n} \int \frac{d^{d-1} p}{(2 \pi)^{d-1}}\left\{\log \left[-\beta^{2}\left(g^{00} p_{0}^{2}+2 g^{0 i} p_{0} p_{i}+g^{i j} p_{i} p_{j}\right)\right]+\log (\sqrt{-g})\right\},
$$

onde escrevemos a métrica em termos de suas componentes. Usando a regularização zeta, vemos que os termos independentes do momento se anulam pois

$$
\sum_{n=-\infty}^{\infty} 1 \approx \lim _{\delta \rightarrow 0} \sum_{n=-\infty}^{\infty} \frac{1}{n^{\delta}}=\lim _{\delta \rightarrow 0}[1+2 \zeta(\delta)] \rightarrow 0
$$

onde usamos que $\zeta(0)=-1 / 2$. Portanto escrevemos

$$
\mathcal{L}^{\text {stat. }}=-\frac{T}{2} \sum_{n} \int \frac{d^{d-1} p}{(2 \pi)^{d-1}} \log \left[-\beta^{2}\left(g^{00} p_{0}^{2}+2 g^{0 i} p_{0} p_{i}+g^{i j} p_{i} p_{j}\right)\right] .
$$


Vamos agora fazer um mudança de variáveis na forma

$$
p_{i} \rightarrow p_{i}^{\prime}=M_{i j} p_{j}+f_{i} p_{0}
$$

onde $M$ é uma matriz simétrica. Impondo a condição

$$
p_{i}^{\prime} p_{i}^{\prime}=g^{i j} p_{i} p_{j}+2 g^{0 i} p_{0} p_{i}+f_{i} f_{i} p_{0}^{2}
$$

obtemos

$$
\left\{\begin{array}{l}
M_{i}{ }^{j} M_{i}^{k}=g^{j k} \\
f_{i} M_{i}^{j}=g^{0 j}
\end{array} .\right.
$$

Como a matriz $M$ é simétrica por hipótese, a primeira linha de 7.28 implica que o jacobiano da transformação (7.26) tem a forma

$$
J=\left|\frac{\partial p_{i}}{\partial p_{j}^{\prime}}\right|=\frac{1}{\sqrt{\operatorname{det} \mathbf{g}}}
$$

onde $\mathbf{g}$ representa a matriz com componentes $g^{i j}$, isto é, é a parte espacial da métrica. A segunda linha de 7.28 implica em

$$
f_{i}=\left(M^{-1}\right)_{i j} g^{0 j}
$$

de tal maneira que, usando novamente a primeira linha de 7.28 , teremos

$$
f_{i} f_{i}=\left(M^{-1}\right)_{i j} g^{0 j}\left(M^{-1}\right)_{i k} g^{0 k}=\left(\mathbf{g}^{-1}\right)_{j k} g^{0 j} g^{0 k}
$$

onde $\left(\mathbf{g}^{-1}\right)_{j k}$ representa a inversa da parte espacial da métrica, isto é, $g^{i j}\left(\mathbf{g}^{-1}\right)_{j k}=\delta_{k}^{i}$.

Portanto, a lagrangiana efetiva pode ser escrita como

$$
\mathcal{L}^{\text {stat. }}=-\frac{T}{2} \frac{1}{\sqrt{\operatorname{detg}}} \sum_{n} \int \frac{d^{d-1} p^{\prime}}{(2 \pi)^{d-1}} \log \left\{-\beta^{2}\left[\left(g^{00}-f^{i} f^{i}\right) p_{0}^{2}+p_{i}^{\prime} p_{j}^{\prime}\right]\right\},
$$

onde as entradas da matriz g são $g^{i j}$. Fazendo a transformação

$$
p_{i} \rightarrow \sqrt{\frac{g^{00}-f^{j} f^{j}}{-1}} p_{i}
$$

obtemos

$$
\mathcal{L}^{\text {stat. }}=\mathcal{L}^{(0)} \frac{\left(g^{00}-\left(\mathbf{g}^{-1}\right)_{i j} g^{0 i} g^{0 j}\right)^{\frac{d-1}{2}}}{\sqrt{(-1)^{d-1} \operatorname{det} \mathbf{g}}} .
$$

Podemos verificar de maneira simples que a lagrangiana efetiva (7.34 de fato gera os 
resultados perturbativos da seção passada. De fato, usando a equação (7.4), a contribuição de primeira ordem vinda da equação (7.34) é simplesmente

$$
\mathcal{L}^{(1)}=\frac{\mathcal{L}^{(0)}}{2}\left(d \tilde{h}^{00}-\tilde{h}\right)
$$

que é o mesmo termo de primeira ordem (7.13). De manira análoga, para o termo de segunda ordem de 7.34 possui a forma

$$
\mathcal{L}^{(2)}=\frac{1}{8} \mathcal{L}^{(0)}\left[d(d-2)\left(\tilde{h}^{00}\right)^{2}-2 d \tilde{h}^{00} \tilde{h}+\tilde{h}^{2}+2\left(\tilde{h}_{\mu \nu} \tilde{h}^{\mu \nu}\right)_{\tilde{h}_{0 i}=0}-4(d-1) \tilde{h}_{0 i} \tilde{h}^{0 i}\right],
$$

que também está de acordo com o resultado de segunda ordem (7.13). Novamente, em virtude da invariância conforme da lagrangiana efetiva, o resultado é invariante por $g^{\mu \nu} \rightarrow$ $\tilde{g}^{\mu \nu} \equiv \sqrt{-g} g^{\mu \nu}$, como pode ser antecipado de 7.22 .

Podemos expressar o resultado exato (7.34) de uma maneira explicitamente covariante nas componentes da métrica. Usando a identidade $g^{\mu \alpha} g_{\alpha \nu}=\delta_{\nu}^{\mu}$ é imediato que

$$
g^{i 0} g_{00}+g^{i j} g_{j 0}=0
$$

e

$$
g^{00} g_{00}+g^{0 i} g_{i 0}=1
$$

Contraindo 7.37 com $\left(\mathbf{g}^{-1}\right)_{k i}$ implica em

$$
g_{i 0}=-\left(\mathbf{g}^{-1}\right)_{i k} g^{k 0} g_{00}
$$

e substituindo 7.39 em 7.38 , obtemos

$$
g^{00}-\left(\mathbf{g}^{-1}\right)^{i j} g^{0 i} g^{0 j}=\left(g_{00}\right)^{-1}
$$

Substituindo 7.40 em 7.34 teremos

$$
\mathcal{L}^{\text {stat. }}=\mathcal{L}^{(0)} \frac{\sqrt{(-1)^{d-1} g_{00} \operatorname{det} \mathbf{g}^{-1}}}{\left(g_{00}\right)^{d / 2}} .
$$

Para chegar a uma forma mais compacta, vamos expandir o determinante em termos dos cofatores na forma

$$
\operatorname{det} g^{\mu \nu}=\sum_{\mu} C^{0 \mu} g^{0 \mu}=C^{00} g^{00}+\sum_{i} C^{0 i} g^{0 i}
$$

Da álgebra linear, sabemos que o cofator pode ser escrito em termos do determinante da 
submatriz,

$$
C^{00}=(-1)^{0+0} \operatorname{det} g^{i j}=\operatorname{det} \mathbf{g}
$$

ou em termos do elemento transposto da inversa

$$
C^{0 i}=\operatorname{det}\left(g^{\mu \nu}\right) g_{i 0}=-\operatorname{det}\left(g^{\mu \nu}\right)\left(\mathbf{g}^{-1}\right)_{i k} g^{k 0} g_{00},
$$

onde usamos (7.39). Deste modo, substituindo (7.43) e (7.44) em (7.42) e isolando det $g^{\mu \nu}$ teremos

$$
\operatorname{det} g^{\mu \nu}=\frac{g^{00} \operatorname{det} \mathbf{g}}{1+\left(\mathbf{g}^{-1}\right)_{i j} g^{0 i} g^{j 0} g_{00}}=\frac{\operatorname{det} \mathbf{g}}{g_{00}},
$$

onde, na última igualdade foi usado o resultado 7.40 . Invertendo 7.45 obtemos

$$
\operatorname{det} g_{\mu \nu}=g_{00} \operatorname{det} \mathbf{g}^{-1}
$$

Portanto, a equação (7.41) fornece

$$
\mathcal{L}^{\text {stat. }}=\mathcal{L}^{(0)} \frac{\sqrt{-g}}{\left(g_{00}\right)^{d / 2}}
$$

que é consistente com o resultado em $d=4$ [Reb91]. Esta lagrangiana efetiva pode ser obtida usando a técnica dos núcleos de calor!̣ restrita a um espaço-tempo estático, em um referencial em que $g_{0 i}=d^{2}$ dAO95].

Como presença do banho térmico quebra a invariância por transformações gerais de coordenadas, o que é evidente devido a presença da soma de Matsubara na equação $7.22]^{3}$, é fisicamente relevante computar a ação efetiva para valores gerais de $g_{0 i}$, tal como considerado aqui. Supondo que o banho térmico está livre da ação de forças, temos que impor que o banho térmico está se movendo sobre uma geodésica tipo tempo, assim no referencial de repouso do banho térmico, $g_{0 i}$ não se anula em geral, mesmo para espaçostempos estáticos.

\footnotetext{
1 Tradução livre de "heat-kernel".

${ }^{2} \mathrm{Na}$ nomenclatura usual de relatividade geral d'I92, um espaço-tempo é dito estático se existe um sistema de coordenadas em que a métrica é independente do tempo e $g_{0 i}=0$, o que é equivalente a dizer que o espaço-tempo admite um vetor de Killing tipo tempo. No contexto do presente trabalho dizemos que a métrica é estática se ela não depende da variável temporal.

${ }^{3} \mathrm{O}$ fato da soma em 7.22 se dar sobre $p_{0}$ implica que estamos no referencial de repouso do banho térmico.
} 


\subsection{QED em um campo gravitacional estático}

\subsubsection{Definição do modelo}

Nesta seção vamos considerar a QED na presença de uma métrica de fundo estática, e as mesmas considerações sobre o campo gravitacional feitas na seção passada também se aplicam aqui. Portanto, para obter a ação efetiva estática do campo gravitacional no limite de altas temperaturas, podemos considerar configurações de campo constantes (veja capítulo 5). Assim, consideraremos a QED com uma métrica de fundo constante para obter a ação efetiva estática do campo gravitacional interagindo com um plasma da QED, no limite de altas temperaturas.

Para uma métrica de fundo constante, temos que a conexão de spin, definida no apêndice G [veja a equação G.92], se anula. Logo, a derivada covariante para qualquer representação do grupo de Lorentz [veja equação (G.111)] coincide com a derivada ordinária. Portanto, a única modificação na lagrangiana será a mudança $\eta_{\mu \nu} \rightarrow g_{\mu \nu}$. Por consistência, devemos mudar também a álgebra de Clifford

$$
\left\{\bar{\gamma}^{\mu}, \bar{\gamma}^{\nu}\right\}=2 g^{\mu \nu}
$$

para que a equação de Dirac livre seja consistente com a equação de Kein-Gordon, a menos de correções de curvatura [PT09]. As matrizes $\bar{\gamma}^{\mu}$ são as matrizes de Dirac modificadas pela presença da métrica.

Usaremos a mesma notação do apêndice G, segundo a qual os índices $\mu, \nu, \cdots$ representam índices de transformações gerais de coordenadas enquanto $a, b, \cdots$ representam índices de Lorentz locais. Portanto, os índices $\mu, \nu, \cdots$ são levantados e abaixados pela métrica $g_{\mu \nu}$, enquanto os índices $a, b, \cdots$ possuem o produto escalar dado pela métrica $\eta_{a b}$.

Depois destas considerações, a densidade lagrangiana do modelo no calibre de Feynman será

$$
\mathcal{L}=\sqrt{-g}\left[i \bar{\psi} g^{\mu \nu} \bar{\gamma}_{\mu}\left(\partial_{\nu}-i e A_{\nu}\right) \psi-\frac{1}{4} g^{\mu \nu} g^{\alpha \beta} F_{\mu \alpha} F_{\nu \beta}-\frac{1}{2}\left(g^{\mu \nu} \partial_{\mu} A_{\nu}\right)^{2}+g^{\mu \nu} \partial_{\mu} \bar{C} \partial_{\nu} C\right] .
$$

No formalismo das tetradas temos

$$
g^{\mu \nu}=E_{a}{ }^{\mu} E_{b}{ }^{\nu} \eta^{a b}
$$



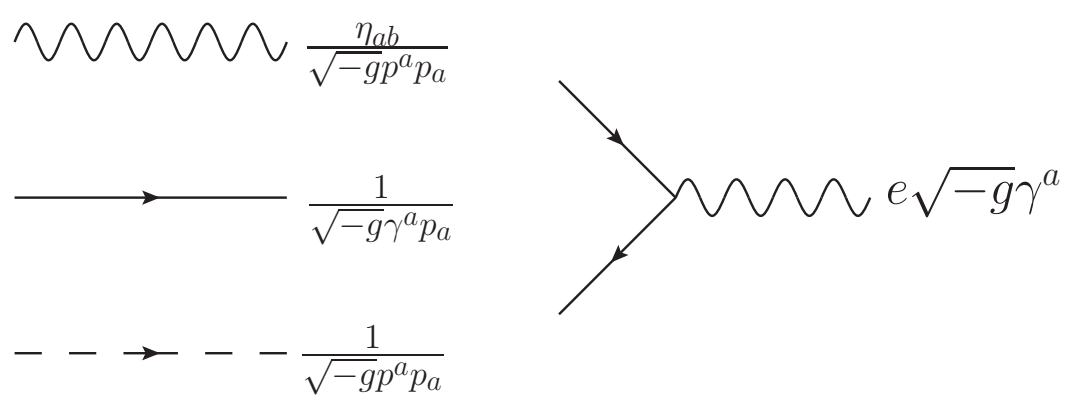

Figura 7.1: Regras de Feynman

e para todos os vetores usaremos a notação

$$
g^{\mu \nu} \bar{\gamma}_{\mu} \partial_{\nu}=\eta^{a b} \gamma_{a} \partial_{b}
$$

onde

$$
\gamma_{a}=E_{a}{ }^{\mu} \bar{\gamma}_{\mu}
$$

A álgebra das matrizes $\gamma_{a}$ pode ser obtida de (7.48) como segue

$$
\left\{\gamma_{a}, \gamma_{b}\right\}=2 E_{a}{ }^{\mu} E_{b}{ }^{\nu} g_{\mu \nu}=2 E_{a}{ }^{\mu} E_{b}{ }^{\nu} e^{c}{ }_{\mu} e^{d}{ }_{\nu} \eta_{c d}=2 \eta_{a b}
$$

onde usamos a definição da tetrada G.58 e o fato que a matriz $E_{a}{ }^{\mu}$ é a inversa de $e^{a}{ }_{\mu}$ [veja as equações G.54 e G.55]. Logo, $\gamma_{a}$ satisfazem a álgebra de Clifford usual e são uma representação das matrizes de Dirac.

Na notação das tetradas a lagrangiana (7.49) assume a forma

$$
\mathcal{L}=\sqrt{-g}\left[i \bar{\psi} \gamma^{a}\left(\partial_{a}-i e A_{a}\right) \psi-\frac{1}{4} F_{a b} F^{a b}-\frac{1}{2}\left(\partial_{a} A^{a}\right)^{2}+\partial_{a} \bar{C} \partial^{a} C\right]
$$

Considerando $A^{a}$ como campo quântico no formalismo da integração funciona $\rrbracket^{4}$ obtemos as regras de Feynman para o modelo que estão expressas na figura 7.1 .

\subsubsection{Lagrangiana efetiva em ordem de 1 laço}

Os diagramas que contribuem para a pressão em ordem 1 laço estão expressos na figura 7.2. Usando a correspondência entre a lagrangiana efetiva estática e a pressão como função do campo constante, obtemos a seguinte contribuição de ordem de um laço

\footnotetext{
${ }^{4}$ Definindo um observador como um ente que viaja sobre uma geodésica tipo tempo, dotado de um sistema de coordenadas localmente inercial, vemos que o campo projetado na base da tetrada é a escolha mais natural para campo físico.
} 


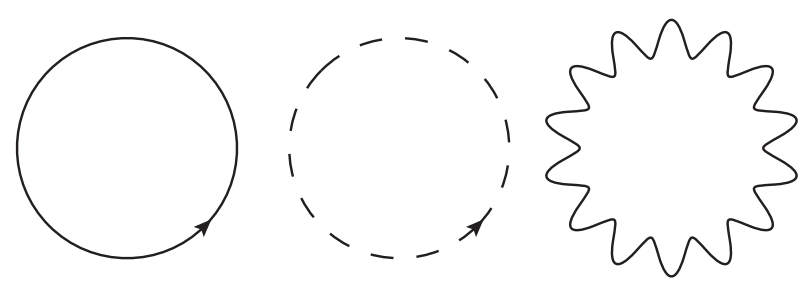

Figura 7.2: Pressão ordem 1 laço

para a lagrangiana efetiva

$$
\begin{aligned}
\mathcal{L}_{1} & =T \sum_{p_{0}} \int \frac{d^{d-1} p}{(2 \pi)^{d-1}} \operatorname{tr} \log \left(\beta g^{\mu \nu} \bar{\gamma}_{\mu} p_{\nu}\right)+T \sum_{q_{0}} \int \frac{d^{d-1} q}{(2 \pi)^{d-1}} \log \left(-\beta^{2} g^{\mu \nu} q_{\mu} q_{\nu}\right) \\
& +d\left(-\frac{1}{2}\right) T \sum_{q_{0}} \int \frac{d^{d-1} q}{(2 \pi)^{d-1}} \log \left(-\beta^{2} g^{\mu \nu} q_{\mu} q_{\nu}\right),
\end{aligned}
$$

onde a primeira soma-integral representa a contribuição do férmions, a segunda a contribuição dos fantasmas e a última a contribuição dos fótons. Desta maneira, $p_{0}=$ $i(2 n+1) \pi T$ é uma frequência de Matsubara de um férmion, enquanto $q_{0}=i 2 n \pi T$ é a frequência de Matsubara de um bóson.

Para a contribuição dos férmions é mais simples fazer a álgebra das matrizes de Dirac na base das tetradas, obtendo

$$
\begin{aligned}
\mathcal{L}_{1}^{F} & =T \sum_{p_{0}} \int \frac{d^{d-1} p}{(2 \pi)^{d-1}} \operatorname{tr} \log \left(\beta \gamma^{a} p_{a}\right) \\
& =\frac{T}{2} \sum_{p_{0}} \int \frac{d^{d-1} p}{(2 \pi)^{d-1}} \operatorname{tr} \log \left(\beta^{2} \gamma^{a} \gamma^{b} p_{a} p_{b}\right) \\
& =\frac{T}{2} \sum_{p_{0}} \int \frac{d^{d-1} p}{(2 \pi)^{d-1}} \operatorname{tr} \log \left(\beta^{2} \eta^{a b} p_{a} p_{b}\right) \\
& =\frac{T}{2} \sum_{p_{0}} \int \frac{d^{d-1} p}{(2 \pi)^{d-1}} \operatorname{tr} \log \left(\beta^{2} g^{\mu \nu} p_{\mu} p_{\nu}\right) .
\end{aligned}
$$

Vamos abrir em componentes da métrica e usar que $\operatorname{tr} I=2^{E(d / 2)}$, onde $E(d / 2)$ é a dimensão das matrizes de Dirac, que é o maior inteiro menor ou igual a $d / 2$, para escrever

$$
\mathcal{L}_{1}^{F}=\frac{T}{2} 2^{E(d / 2)} \sum_{p_{0}} \int \frac{d^{d-1} p}{(2 \pi)^{d-1}} \log \left\{\beta^{2}\left[g^{00}\left(p_{0}\right)^{2}+2 g^{0 i} p_{0} p_{i}+g^{i j} p_{i} p_{j}\right]\right\} .
$$

Fazendo as mesmas manipulações algébricas que levaram de 7.22 até 7.41 obtemos

$$
\mathcal{L}_{1}^{F}=2^{E(d / 2)} \frac{\sqrt{-g}}{g_{00}^{d / 2}} \frac{T}{2} \sum_{p_{0}} \int \frac{d^{d-1} p}{(2 \pi)^{d-1}} \log \left\{-\beta^{2}\left[\left(p_{0}\right)^{2}-p_{i} p_{i}\right]\right\},
$$


onde $g=(-1)^{d} \operatorname{det} g_{\mu \nu}$. A soma-integral que restou é contribuição para pressão vinda dos férmions no espaço de Minkowski d-dimensional. Podemos avaliar esta soma-integral de maneira usual (veja seção 4.2) obtendo

$$
2^{E(d / 2)} \frac{T}{2} \sum_{p_{0}} \int \frac{d^{d-1} p}{(2 \pi)^{d-1}} \log \left\{-\beta^{2}\left[\left(p_{0}\right)^{2}-p_{i} p_{i}\right]\right\}=T^{d} \frac{\Gamma(d) \zeta(d) 2^{E(d / 2)}\left(1-2^{1-d}\right)}{(2 \sqrt{\pi})^{d-1} \Gamma\left(\frac{d+1}{2}\right)},
$$

de modo que obtemos a contribuição dos férmions para a pressão em ordem um laço como sendo

$$
\mathcal{L}_{1}^{F}=\frac{\sqrt{-g}}{g_{00}^{d / 2}}\left[T^{d} \frac{\Gamma(d) \zeta(d) 2^{E(d / 2)}\left(1-2^{1-d}\right)}{(2 \sqrt{\pi})^{d-1} \Gamma\left(\frac{d+1}{2}\right)}\right],
$$

onde $\Gamma$ é a função gama de Euler, $\zeta$ é a função zeta de Rienman.

A contribuição dos bósons para a pressão pode ser escrita como

$$
\begin{aligned}
\mathcal{L}_{1}^{B} & =\frac{2-d}{2} T \sum_{q_{0}} \int \frac{d^{d-1} q}{(2 \pi)^{d-1}} \log \left(-\beta^{2} q^{a} q_{a}\right) \\
& =\frac{2-d}{2} T \sum_{q_{0}} \int \frac{d^{d-1} q}{(2 \pi)^{d-1}} \log \left(-\beta^{2} g^{\mu \nu} q_{\mu} q_{\nu}\right) .
\end{aligned}
$$

Repetindo as mudanças de variáveis que levaram de 7.22 à 7.41 obtemos

$$
\mathcal{L}_{1}^{B}=\frac{\sqrt{-g}}{g_{00}^{d / 2}}\left[\frac{2-d}{2} T \sum_{q_{0}} \int \frac{d^{d-1} q}{(2 \pi)^{d-1}} \log \left(-\beta^{2} \eta^{\mu \nu} q_{\mu} q_{\nu}\right)\right]
$$

em que a quantidade entre chaves é a pressão dos bósons no espaço de Minkowski, que pode ser obtida com técnicas usuais (veja seção 4.2), como

$$
\frac{2-d}{2} T \sum_{q_{0}} \int \frac{d^{d-1} q}{(2 \pi)^{d-1}} \log \left(-\beta^{2} \eta^{\mu \nu} q_{\mu} q_{\nu}\right)=(d-2) T^{d} \frac{\Gamma(d) \zeta(d)}{(2 \sqrt{\pi})^{d-1} \Gamma\left(\frac{d+1}{2}\right)},
$$

onde estamos desprezando a energia do ponto zero do oscilador harmônico. Logo, a pressão dos bósons livres será

$$
\mathcal{L}_{1}^{B}=\frac{\sqrt{-g}}{g_{00}^{d / 2}}(d-2) T^{d} \frac{\Gamma(d) \zeta(d)}{(2 \sqrt{\pi})^{d-1} \Gamma\left(\frac{d+1}{2}\right)} .
$$

Juntando os resultados 7.64 e 7.60 obtemos a lagrangiana efetiva em ordem um laço

$$
\mathcal{L}_{1}=\frac{\sqrt{-g}}{g_{00}^{d / 2}} T^{d} \frac{\Gamma(d) \zeta(d)}{(2 \sqrt{\pi})^{d-1} \Gamma\left(\frac{d+1}{2}\right)}\left[(d-2)+2^{E(d / 2)}\left(1-2^{1-d}\right)\right]
$$




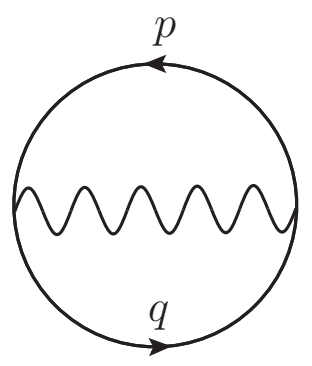

Figura 7.3: Pressão ordem 2 laços

\subsubsection{Lagrangiana efetiva em ordem de 2 laços}

O diagrama que contribui para a lagrangiana efetiva em ordem 2 laços está representado na figura 7.3, e possui expressão analítica dada por

$$
\mathcal{L}_{2}=\frac{e^{2} T^{2}}{2} \sum_{p_{0} q_{0}} \int \frac{d^{d-1} p}{(2 \pi)^{d-1}} \frac{d^{d-1} q}{(2 \pi)^{d-1}} \operatorname{tr}\left(\bar{\gamma}^{\mu} \frac{1}{\bar{\gamma}^{\nu} p_{\nu}} \bar{\gamma}_{\mu} \frac{1}{\bar{\gamma}^{\alpha} p_{\alpha}}\right) \frac{1}{\sqrt{-g}(p-q)^{\gamma}(p-q)_{\gamma}},
$$

onde $q_{0}$ e $p_{0}$ são frequências de Matsubara fermiônicas. Novamente, é conveniente fazer a álgebra das matrizes de Dirac na base das tetradas. Usando

$$
\frac{1}{\gamma^{a} p_{a}}=\frac{\gamma^{a} p_{a}}{p^{c} p_{c}}
$$

reescrevemos

$$
\mathcal{L}_{2}=\frac{e^{2} T^{2}}{2 \sqrt{-g}} \sum_{p_{0} q_{0}} \int \frac{d^{d-1} p}{(2 \pi)^{d-1}} \frac{d^{d-1} q}{(2 \pi)^{d-1}} \operatorname{tr}\left(\gamma^{a} \gamma^{b} \gamma_{a} \gamma^{d}\right) \frac{p_{b} q_{d}}{\left(p^{b} p_{b}\right)\left(q^{c} q_{c}\right)(p-q)^{f}(p-q)_{f}} .
$$

Usando a álgebra de Clifford (7.53), podemos avaliar o traço das matrizes de Dirac de maneira usual como

$$
\operatorname{tr}\left(\gamma^{a} \gamma^{b} \gamma_{a} \gamma^{d}\right)=2^{E(d / 2)}(2-d) \eta^{b d}
$$

de modo que teremos

$$
\mathcal{L}_{2}=\frac{e^{2} T^{2}}{2 \sqrt{-g}} 2^{E(d / 2)}(2-d) \sum_{p_{0} q_{0}} \int \frac{d^{d-1} p}{(2 \pi)^{d-1}} \frac{d^{d-1} q}{(2 \pi)^{d-1}} \frac{p^{a} q_{a}}{\left(p^{b} p_{b}\right)\left(q^{c} q_{c}\right)(p-q)^{d}(p-q)_{d}} .
$$

Lançando mão da identidade algébrica

$$
p^{a} q_{a}=\frac{1}{2}\left[p^{a} p_{a}+q^{a} q_{a}-(p-q)^{a}(p-q)_{a}\right],
$$


obtemos

$$
\begin{aligned}
\mathcal{L}_{2} & =\frac{e^{2} T^{2}}{4 \sqrt{-g}} 2^{E(d / 2)}(2-d) \sum_{p_{0} q_{0}} \int \frac{d^{d-1} p}{(2 \pi)^{d-1}} \frac{d^{d-1} q}{(2 \pi)^{d-1}}\left[\frac{1}{\left(p^{a} p_{a}\right)(p-q)^{b}(p-q)_{b}}\right. \\
& \left.+\frac{1}{\left(q^{a} q_{a}\right)(p-q)^{b}(p-q)_{b}}-\frac{1}{\left(p^{a} p_{a}\right)\left(q^{b} q_{b}\right)}\right] \\
& =\frac{e^{2} 2^{E(d / 2)}(2-d)}{4 \sqrt{-g}} 2^{E(d / 2)}(2-d)\left[2 T^{2} \sum_{p_{0} k_{0}} \int \frac{d^{d-1} p}{(2 \pi)^{d-1}} \frac{d^{d-1} k}{(2 \pi)^{d-1}} \frac{1}{\left(p^{a} p_{a}\right)\left(k^{a} k_{a}\right)}\right. \\
& \left.-T^{2} \sum_{p_{0} q_{0}} \int \frac{d^{d-1} p}{(2 \pi)^{d-1}} \frac{d^{d-1} q}{(2 \pi)^{d-1}} \frac{1}{\left(p^{a} p_{a}\right)\left(q^{b} q_{b}\right)}\right]
\end{aligned}
$$

Em 7.72 as integrais se fatoram, de modo que temos que tratar duas integrais simples

$$
I_{1}=T \sum_{p_{0}} \int \frac{d^{d-1} p}{(2 \pi)^{d-1}} \frac{1}{g^{\mu \nu} p_{\mu} p_{\nu}}
$$

e

$$
I_{2}=T \sum_{k_{0}} \int \frac{d^{d-1} k}{(2 \pi)^{d-1}} \frac{1}{g^{\mu \nu} k_{\mu} k_{\nu}},
$$

onde $p_{0}$ é a frequências de Matsubara de férmions, enquanto $k_{0}$ é uma frequência de Matsubara de um bóson. Vamos começar tratando $I_{1}$. Abrindo em componentes do tensor métrico, temos

$$
I_{1}=T \sum_{p_{0}} \int \frac{d^{d-1} p}{(2 \pi)^{d-1}} \frac{1}{g^{00}\left(p_{0}\right)^{2}+2 g^{0 i} p_{0} p_{i}+g^{i j} p_{i} p_{j}}
$$

fazendo a mudança de variáveis (7.26) e usando (7.40) e (7.46), teremos

$$
\begin{aligned}
I_{1} & =\frac{1}{\sqrt{(-1)^{d-1} \operatorname{det} \mathbf{g}}} T \sum_{p_{0}} \int \frac{d^{d-1} p}{(2 \pi)^{d-1}} \frac{1}{\left(g_{00}\right)^{-1} p_{0}^{2}-|\vec{p}|^{2}} \\
& =\sqrt{\frac{g_{00}}{(-1)^{d-1} \operatorname{det} \mathbf{g}}}\left(g_{00}\right)^{1-d / 2} T \sum_{p_{0}} \int \frac{d^{d-1} p}{(2 \pi)^{d-1}} \frac{1}{p_{0}^{2}-|\vec{p}|^{2}} \\
& =\frac{\sqrt{-g}}{\left(g_{00}\right)^{d / 2-1}} T \sum_{p_{0}} \int \frac{d^{d-1} p}{(2 \pi)^{d-1}} \frac{1}{p_{0}^{2}-|\vec{p}|^{2}} .
\end{aligned}
$$

A integral resultante já foi tratada na seção 4.3, de modo que a parte térmica possui a forma

$$
I_{1}=\frac{\sqrt{-g}}{\left(g_{00}\right)^{d / 2-1}} T^{d-2}\left(2-2^{4-d}\right) \frac{\Gamma(d-2) \zeta(d-2)}{\Gamma\left(\frac{d-1}{2}\right)(2 \sqrt{\pi})^{d-1}}
$$


Argumentos semelhantes também se aplicam para a integral $I_{2}$, de modo que teremos

$$
\begin{aligned}
I_{2} & =T \sum_{k_{0}} \int \frac{d^{d-1} k}{(2 \pi)^{d-1}} \frac{1}{k^{a} k_{a}} \\
& =\frac{\sqrt{-g}}{\left(g_{00}\right)^{d / 2-1}} T \sum_{k_{0}} \int \frac{d^{d-1} k}{(2 \pi)^{d-1}} \frac{1}{k_{0}^{2}-|\vec{k}|^{2}} \\
& =\frac{\sqrt{-g}}{\left(g_{00}\right)^{d / 2-1}} T^{d-2}(-2) \frac{\Gamma(d-2) \zeta(d-2)}{\Gamma\left(\frac{d-1}{2}\right)(2 \sqrt{\pi})^{d-1}}
\end{aligned}
$$

Com estes resultados, escrevemos a lagrangiana efetiva ordem dois laços é escrita como

$$
\mathcal{L}_{2}=\frac{\sqrt{-g}}{\left(g_{00}\right)^{d-2}} T^{2 d-4} e^{2} 2^{E(d / 2)-2}(2-d)\left[\frac{\Gamma(d-2) \zeta(d-2)}{\Gamma\left(\frac{d-1}{2}\right)(2 \sqrt{\pi})^{d-1}}\right]^{2}\left(2-2^{4-d}\right)\left(6-2^{4-d}\right) .
$$

Este resultado pode ser identificado com a correção em ordem dois laços para a pressão no limite de altas temperaturas. O resultado é idêntico à pressão em ordem dois laços no espaço de Minkowski 4.30, a menos a um fator de $\sqrt{-g}$ devido ao fato da pressão ser uma densidade, e corrigindo a temperatura pela temperatura de Tolman [TE30]. 


\section{Conclusão}

No presente trabalho, estudamos efeitos térmicos em teorias de campos. Primeiro, fizemos uma revisão dos conceito básicos de teoria de campos a temperatura finita Bel96, KG06. Generalizamos para um espaço-tempo $d$-dimensional os principais resultados perturbativos da teoria escalar com interação $\phi^{4}$ e da teoria de calibre da QED.

Nestes contextos, calculamos a soma das divergências infravermelhas para a pressão. Verificamos que o comportamento não-analítico da série perturbativa depende qualitativamente da dimensionalidade do espaço-tempo. Quando $d$ é par a não-analiticidade é do tipo $\lambda^{(d-1) / 2}$, onde $\lambda$ é a constante de acoplamento, enquanto no caso em que $d$ é ímpar a não-analiticidade é apenas logarítmica [BFS12].

No método do campo de fundo a temperatura finita, notamos que a pressão na presença de um campo externo pode ser identificada com a lagrangiana efetiva deste campo. Em seguida, mostramos que no limite de altas temperaturas podemos obter as funções de Green estáticas supondo que todas as componentes do momento externo são nulas, para todas as amplitudes 1PI em ordem de um laço e para a auto-energia em ordem de dois laços [BS12b, FPT09]. Aplicado ao método do campo de fundo, este resultado implica que podemos obter a lagrangiana de um campo externo estático calculando a pressão na presença de um campo de fundo constante, no limite de altas temperaturas.

Aplicamos este método em três contextos distintos. Obtivemos o limite de altas temperaturas da lagrangiana efetiva do campo gravitacional interagindo com partículas escalares térmicas [BS12a], em ordem de um laço. Consideremos a QED na presença de campos externos em dois contextos distintos. Primeiro consideramos um plasma da QED na presença de um campo de Maxwell externo, e obtivemos a lagrangiana efetiva estática do campo de Maxwell até ordem de dois laços, no limite de altas temperaturas. Depois consideramos um plasma da QED interagindo com um campo gravitacional externo, e obtivemos a lagrangiana efetiva estática do campo gravitacional até ordem de dois laços, no limite de altas temperaturas. Os resultados da QED na presença de campo externos 
ainda não foram publicados e estão em fase de preparação. 


\section{APÊEDICE A - Integrais funcionais}

Nesta seção, seguiremos [KG06] e definiremos a integração funcional na rede e no caso de uma representação em uma base do espaços das funções, tal como a base de Fourier.

Na rede, dividimos o espaço em células de volume $\Delta v_{l}$ e as integrais são escritas em termos de somas de Riemann como

$$
I[f]=g\left[\int d^{d-1} x f(\vec{x})\right]=\left[\sum_{l} \Delta v_{l} f\left(\vec{x}_{l}\right)\right] .
$$

Neste caso, a integração funcional de $I[f]$ possui a forma

$$
Z=\int \mathcal{D} f I[f]=\prod_{m} \int_{-\infty}^{\infty} d f\left(\vec{x}_{m}\right) g\left[\sum_{l} \Delta v_{l} f\left(\vec{x}_{l}\right)\right],
$$

e a generalização para funcionais mais gerais é imediata.

Muitas vezes, a expressão na rede não é o meio mais conveniente de expressar os funcionais em teorias de campos. Como veremos no decorrer do texto, a base de Fourier diagonaliza a parte quadrática da ação. Motivado por isso, consideremos o caso em que escrevemos os campos em uma base do espaço das funções, tal como

$$
f(\vec{x})=\sum_{k} f_{k} h_{k}(\vec{x})
$$

onde os $h_{k}(\vec{x})$ formam um conjunto completo. O índice $k$ pode eventualmente ser contínuo, com a soma recaindo em uma integral. Nesta expansão, o funcional $I$ assume a forma

$$
I[f]=g\left[\sum_{k} f_{k} \int d^{d-1} x h_{k}(\vec{x})\right] .
$$

Neste contexto, variar a forma funcional de $f$ é equivalente a variar os coeficientes da expansão $f_{k}$. Logo, nesta base, a integração funcional é escrita como

$$
Z=\int \mathcal{D} f I[f]=\prod_{q} \int_{-\infty}^{\infty} d f_{q} g\left[\sum_{k} f_{k} \int d^{d-1} x h_{k}(\vec{x})\right]
$$


e novamente a generalização para funcionais mais gerais é imediata. 


\section{APÊNDICE B - Variáveis de Grassmann}

\section{B.1 Integrais gaussianas}

As componentes do campo de Dirac satisfazem regras de anti-comutação de tempos iguais

$$
\left\{\psi_{a}(\vec{x}), \psi_{b}^{\dagger}(\vec{y})\right\}=\hbar \delta_{a b} \delta(\vec{x}-\vec{y})
$$

e

$$
\left\{\psi_{a}(\vec{x}), \psi_{b}(\vec{y})\right\}=\left\{\psi_{a}^{\dagger}(\vec{x}), \psi_{b}^{\dagger}(\vec{y})\right\}=0
$$

Deste modo, no limite clássico $(\hbar \rightarrow 0)$, o campo de Dirac é descrito por variáveis anticomutantes ou variáveis de Grassmann.

Considere um conjunto de variáveis $\theta_{i}$ e $\theta_{i}^{\dagger}$ que satisfazem a álgebra

$$
\left\{\theta_{i}, \theta_{j}\right\}=\left\{\theta_{i}, \theta_{j}^{\dagger}\right\}=\left\{\theta_{i}^{\dagger}, \theta_{j}^{\dagger}\right\}=0
$$

e em particular teremos que

$$
\theta_{i}^{2}=0
$$

Assim, assumindo que as funções de variáveis de Grassmann admitem uma expansão em série de Taylor, obtemos que a forma mais geral para uma função de uma variável de Grassmann $f(\theta)$ possui a forma

$$
f(\theta)=a+b \theta
$$

A integração sobre variáveis de Grassmann é definida com regras idênticas a da operação de derivada

$$
\int d \theta=0
$$


e

$$
\int d \theta \theta=1
$$

de modo que teremos

$$
\int d \theta f(\theta)=b
$$

Para uma função de $N$ variáveis de Grassmann $f\left(\theta_{i}\right)$, para $i=1, \cdots, N$, a série de Taylor terá a forma

$$
f\left(\theta_{i}\right)=a+\sum_{i} b_{i} \theta_{i}+\cdots+d \theta_{1} \cdots \theta_{N}
$$

ou seja, irá até ordem $N$.

A integral mais importante que teremos será a integral Gaussinana. O caso mais simples será

$$
J_{1}=\int d \theta^{\dagger} d \theta e^{-\theta^{\dagger} \lambda \theta}
$$

de modo que teremos

$$
J_{1}=\int d \theta^{\dagger} d \theta\left(1-\theta^{\dagger} \lambda \theta\right)
$$

O primeiro temos entre parênteses se anula usando B.6 e o segundo termo fornece

$$
J_{1}=-\int d \theta^{\dagger} d \theta \theta^{\dagger} \lambda \theta=\lambda
$$

Considerando $M_{i j}$ uma matriz $2 \times 2$, temos

$$
J_{2}=\int d \theta_{1}^{\dagger} d \theta_{1} \theta_{2}^{\dagger} d \theta_{2} \exp \left(-\sum_{i, j} \theta_{i}^{\dagger} M_{i j} \theta_{j}\right)
$$

expandindo em Taylor e usando (B.6) obtemos

$$
J_{2}=\int d \theta_{1}^{\dagger} d \theta_{1} \theta_{2}^{\dagger} d \theta_{2} \frac{1}{2}\left(-\sum_{i, j} \theta_{i}^{\dagger} M_{i j} \theta_{j}\right)^{2},
$$

e usando que $\theta_{i}^{2}=0$, obtemos

$$
\begin{aligned}
J_{2}=\int d \theta_{1}^{\dagger} d \theta_{1} \theta_{2}^{\dagger} d \theta_{2} \frac{1}{2} & \left(\theta_{1}^{\dagger} M_{11} \theta_{1} \theta_{2}^{\dagger} M_{22} \theta_{2}+\theta_{1}^{\dagger} M_{11} \theta_{1} \theta_{2}^{\dagger} M_{22} \theta_{2}\right. \\
& \left.+\theta_{1}^{\dagger} M_{12} \theta_{2} \theta_{2}^{\dagger} M_{21} \theta_{1}+\theta_{2}^{\dagger} M_{21} \theta_{1} \theta_{1}^{\dagger} M_{12} \theta_{2}\right)
\end{aligned}
$$


e computando a integral obtemos

$$
J_{2}=\operatorname{det} M
$$

Já no caso $N$ dimensional vamos usar a notação sintética

$$
\int \mathcal{D}\left(\vec{\theta}^{\dagger}, \vec{\theta}\right)=\int d \theta_{1}^{\dagger} d \theta_{1} \cdots d \theta_{N}^{\dagger} d \theta_{N}
$$

e a gaussiana terá a forma

$$
J_{N}=\int \mathcal{D}\left(\overrightarrow{\theta^{\dagger}}, \vec{\theta}\right) \exp \left(-\sum_{i, j=1}^{N} \theta_{i}^{\dagger} M_{i j} \theta_{j}\right),
$$

e usando (B.6) teremos

$$
J_{N}=\int \mathcal{D}\left(\overrightarrow{\theta^{\dagger}}, \vec{\theta}\right) \frac{(-1)^{N}}{N !}\left(\sum_{i, j=1}^{N} \theta_{i}^{\dagger} M_{i j} \theta_{j}\right)^{N} .
$$

Dos termos entre parênteses apenas o termos que possuem todos os índices diferentes irão contribuir, de modo que teremos

$$
J_{N}=\int \mathcal{D}\left(\overrightarrow{\theta^{\dagger}}, \vec{\theta}\right) \frac{(-1)^{N}}{N !}\left(\sum_{\substack{i_{1} \cdots i_{N} \\ \text { permut }}} \sum_{\substack{j_{1} \cdots j_{N} \\ \text { permut }}} \theta_{i_{1}}^{\dagger} M_{i_{1} j_{1}} \theta_{j_{1}} \cdots \theta_{i_{N}}^{\dagger} M_{i_{N} j_{N}} \theta_{j_{N}}\right)
$$

onde a soma

$$
\sum_{\substack{j_{1} \cdots j_{N} \\ \text { permut }}}
$$

se da sobre $j_{1}, \cdots, j_{N}$ sendo todas as permutações de $1, \cdots, N$. Como os pares $\theta_{i}^{\dagger} \theta_{j}$ comutam entre si, isto é

$$
\theta_{i}^{\dagger} \theta_{j} \theta_{l}^{\dagger} \theta_{m}=\theta_{l}^{\dagger} \theta_{m} \theta_{i}^{\dagger} \theta_{j},
$$

a primeira soma sobre as permutações pode ser computada fornecendo

$$
J_{N}=\int \mathcal{D}\left(\overrightarrow{\theta^{\dagger}}, \vec{\theta}\right) \frac{(-1)^{N}}{N !}\left(N ! \sum_{\substack{j_{1} \cdots j_{N} \\ \text { permut }}} \theta_{1}^{\dagger} M_{1 j_{1}} \theta_{j_{1}} \cdots \theta_{N}^{\dagger} M_{N j_{N}} \theta_{j_{N}}\right)
$$


Usando que a acada permutação

$$
\theta_{1}^{\dagger} \theta_{i} \theta_{2}^{\dagger} \theta_{j}=-\theta_{1}^{\dagger} \theta_{j} \theta_{2}^{\dagger} \theta_{i}
$$

iremos ganhar um sinal obtemos,

$$
J_{N}=\int \mathcal{D}\left(\vec{\theta}^{\dagger}, \vec{\theta}\right)(-1)^{N} \theta_{1}^{\dagger} \theta_{1} \cdots \theta_{N}^{\dagger} \theta_{N}\left(\sum_{\substack{j_{1} \cdots j_{N} \\ \text { permut }}}(-1)^{p} M_{1 j_{1}} \cdots M_{N j_{N}}\right),
$$

onde $(-1)^{p}$ é o sinal da permutação. A quantidade entre parênteses é a definição do determinante, de modo que calculando a integral resultante obtemos

$$
J_{N}=\int \mathcal{D}\left(\overrightarrow{\theta^{\dagger}}, \vec{\theta}\right) \exp \left(-\sum_{i, j} \theta_{i}^{\dagger} M_{i j} \theta_{j}\right)=\operatorname{det} M
$$

\section{B.2 Integrais de trajetória para um grau de liberdade fermiônico}

Um oscilador harmônico fermiônico é descrito pelos operadores de criação e aniquilação $\alpha^{\dagger}$ e $\alpha$, que satisfazem as regras de anti-comutação

$$
\left\{\alpha, \alpha^{\dagger}\right\}=1
$$

$\mathrm{e}$

$$
\alpha^{2}=\left(\alpha^{\dagger}\right)^{2}=0 .
$$

A hamiltoniana do oscilador fermiônico possui a forma

$$
H=\hbar \omega\left(\alpha^{\dagger} \alpha-\frac{1}{2}\right),
$$

de tal maneira que os estados

$$
\alpha|0\rangle=0,
$$

e

$$
|1\rangle=\alpha^{\dagger}|0\rangle,
$$


são auto estados da hamiltoniana. Além disso eles são os únicos estados deste espaço de Hilbert pois

$$
\alpha^{\dagger}|1\rangle=0
$$

e

$$
\alpha|1\rangle=\alpha \alpha^{\dagger}|0\rangle=\left\{\alpha, \alpha^{\dagger}\right\}|0\rangle=|0\rangle
$$

e não dispomos de outros operadores para construir mais estados, além do operador número

$$
n=\alpha^{\dagger} \alpha
$$

do qual os estados $|0\rangle$ e $|1\rangle$ são auto vetores com auto valor 0 e 1 respectivamente. Este operador número possui a propriedade de ser idempotente, isto é

$$
n^{2}=\alpha^{\dagger} \alpha \alpha^{\dagger} \alpha=\alpha^{\dagger}\left\{\alpha, \alpha^{\dagger}\right\} \alpha=n
$$

A função de partição pode então ser definida como o traço

$$
Z=\operatorname{tr} e^{-\beta H}
$$

que pode ser calculada de maneira simples como

$$
Z=\left\langle 0\left|e^{-\beta H}\right| 0\right\rangle+\left\langle 1\left|e^{-\beta H}\right| 1\right\rangle=e^{-\beta \hbar \omega / 2}+e^{\beta \hbar \omega / 2}=2 \cosh (\beta \hbar \omega / 2),
$$

entretanto, para aplicações na teoria de campos será útil escrever essa função de partição em na representação de integração funcional.

Para tanto iremos introduzir a noção de estados coerentes. Sejam $c$ e $c^{*}$ números de Grassmann que são anti-comutantes não apenas entre si mas também com os operadores $\alpha$ e $\alpha$ 怆. O estado coerente é definido como

$$
|c\rangle=e^{-c \alpha^{\dagger}}|0\rangle=|0\rangle-c|1\rangle
$$

de tal maneira que

$$
\alpha|c\rangle=-\alpha c|1\rangle=c|0\rangle=c|c\rangle
$$

\footnotetext{
${ }^{1}$ Como os números de Grassmann anti-comutam com os operadores de criação e aniquilação eles serão construídos de modo a comutar com o estado $|1\rangle$ e anti-comutar com o estado $|0\rangle$.
} 
de modo que o "ket" $|c\rangle$ é autovetor do operador de aniquilação $\alpha$

$$
\alpha|c\rangle=c|c\rangle
$$

O "bra" correspondente a este estado será

$$
\langle c|=\langle 0| e^{-\alpha c^{*}}=\langle 0|-\langle 1| c^{*},
$$

de modo que este "bra" é auto estado de $\alpha^{\dagger}$

$$
\langle c| \alpha^{\dagger}=\langle 0| c^{*}=\langle c| c^{*}
$$

com um auto valor $c^{*}$.

Do mesmo modo que no caso bosônico (veja as seções 2.2.1 e 2.2.2), para construir a representação da da função de partição no formalismo funcional, precisamos de representações integrais do traço e de resoluções da unidade. Usando os estados coerentes podemos definir estes objetos usando as propriedades de integração sobre variáveis de Grassmann. Primeiramente, avaliando a integral

$$
\int d c^{*} d c e^{-c^{*} c}|c\rangle\left\langlec \left|=\int d c^{*} d c\left(1-c^{*} c\right)(|0\rangle-c|1\rangle)\left(\langle 0|-\langle 1| c^{*}\right),\right.\right.
$$

usando (B.6) e (B.7), vemos que apenas os termos proporcionais a $c^{*} c$ darão contribuição não nula, e assim teremos

$$
\int d c^{*} d c e^{-c^{*} c}|c\rangle\left\langle c\left|=\int d c^{*} d c\left(-c^{*} c\right)(|0\rangle\langle 0|+| 1\rangle\langle 1|)=\right| 0\right\rangle\langle 0|+| 1\rangle\langle 1|,
$$

e, portanto, chegamos a uma resolução da unidade

$$
\int d c^{*} d c e^{-c^{*} c}|c\rangle\langle c|=1
$$

De maneira análoga, podemos definir o traço para um operado $O$ que comuta $2^{2} \operatorname{com} c$ e $c^{*}$ como

$$
\begin{aligned}
\int d c^{*} d c e^{-c^{*} c}\langle-c|O| c\rangle & =\int d c^{*} d c\left(1-c^{*} c\right)\left(\left\langle0\left|+\langle 1| c^{*}\right) O(|0\rangle-c|1\rangle)\right.\right. \\
& =\int d c^{*} d c\left(-c^{*} c\right)(\langle 0|O| 0\rangle+\langle 1|O| 1\rangle) \\
& =\langle 0|O| 0\rangle+\langle 1|O| 1\rangle \\
& =\operatorname{tr} O
\end{aligned}
$$

\footnotetext{
${ }^{2}$ Qualquer operador bilinear do tipo $\alpha^{\dagger} \alpha$ comuta com $c$ e $c^{*}$, que certamente é o caso da hamiltoniana e do operador número.
} 
Agora vamos construir o formalismo da integração funcional para férmions usando primeiramente $\mathrm{B} .46$ ) para escrever

$$
Z=\operatorname{tr} e^{-\beta H}=\int d c_{0}^{*} d c_{0} e^{-c^{*} c}\left\langle-c_{0}\left|e^{-\beta H}\right| c_{0}\right\rangle
$$

e vamos dividir o intervalo $\hbar \beta$ em $N$ partes de de tamanho $\epsilon$ de modo que teremos

$$
Z=\int d c_{0}^{*} d c_{0} e^{-c_{0}^{*} c_{0}}\left\langle-c_{0}\left|e^{-\frac{1}{\hbar} \epsilon H} \cdots e^{-\frac{1}{\hbar} \epsilon H}\right| c_{0}\right\rangle
$$

com

$$
\hbar \beta=N \epsilon
$$

inserindo $N$ resoluções da unidade B.45 teremos

$$
Z=\left.\int \mathcal{D}\left(c^{*}, c\right) \prod_{i=0}^{N} e^{-c_{i+1}^{*} c_{i+1}}\left\langle c_{i+1}\left|e^{-\frac{1}{\hbar} \epsilon H}\right| c_{i}\right\rangle\right|_{\substack{c_{N+1}=-c_{0} \\ c_{N+1}^{*}=-c_{0}^{*}}}
$$

Resta então avaliar os elementos de matriz

$$
\left\langle c_{i+1}\left|e^{-\frac{1}{\hbar} \epsilon H}\right| c_{i}\right\rangle
$$

Para tanto, note que as equações (B.40) e B.42 implicam em

$$
\left\langle c_{i+1}|H| c_{i}\right\rangle=\left\langle c_{i+1} \mid c_{i}\right\rangle \hbar \omega\left(c_{i+1}^{*} c_{i}-\frac{1}{2}\right)=\left\langle c_{i+1} \mid c_{i}\right\rangle H\left(c_{i+1}^{*}, c_{i}\right),
$$

e usando que

$$
\left\langle c_{i+1} \mid c_{i}\right\rangle=\left(\left\langle0\left|-\langle 1| c_{i+1}^{*}\right)\left(|0\rangle-c_{i}|1\rangle\right)=\left(1+c_{i+1}^{*} c_{i}\right)=e^{c_{i+1}^{*} c_{i}}\right.\right.
$$

vemos que os elementos de matriz possuem a forma

$$
\left\langle c_{i+1}\left|e^{-\frac{1}{\hbar} \epsilon H}\right| c_{i}\right\rangle=\exp \left\{-\frac{1}{\hbar} \epsilon\left[H\left(c_{i+1}^{*}, c_{i}\right)-\hbar \frac{c_{i+1}^{*} c_{i}}{\epsilon}\right]\right\} .
$$

Substituindo B.54 em (B.50, temos

$$
\begin{aligned}
Z & =\int \mathcal{D}\left(c^{*}, c\right) \prod_{i=0}^{N} \exp \left\{-\frac{1}{\hbar} \epsilon\left[H\left(c_{i+1}^{*}, c_{i}\right)+\hbar c_{i+1}^{*} \frac{c_{i+1}-c_{i}}{\epsilon}\right]\right\} \mid \begin{array}{l}
c_{N+1}=-c_{0} \\
c_{N+1}^{*}=-c_{0}^{*} \\
c_{i=1}^{*}
\end{array} \\
& =\left.\int \mathcal{D}\left(c^{*}, c\right) \exp \left\{-\frac{1}{\hbar} \sum_{i=0}^{N} \epsilon\left[H\left(c_{i+1}^{*}, c_{i}\right)+\hbar c_{i+1}^{*} \frac{c_{i+1}-c_{i}}{\epsilon}\right]\right\}\right|_{\substack{c_{N+1}=-c_{0} \\
c_{N+1}^{*}=-c_{0}^{*}}}
\end{aligned}
$$


Deste modo, considerando o limite $N \rightarrow \infty$ obtemos

$$
Z=\left.\int \mathcal{D}\left(c^{*}, c\right) \exp \left\{-\frac{1}{\hbar} \int_{0}^{\beta \hbar} d \tau\left[H\left(c^{*}, c\right)+\hbar c^{*} \frac{\partial c}{\partial \tau}\right]\right\}\right|_{\substack{c(\beta \hbar)=-c(0) \\ c *(\beta \hbar)=-c^{*}(0)}}
$$

que é a representação da função de partição para férmions no formalismo funcional. 


\section{APÊNDICE C - As somas de Matsubara}

Neste apêndice vamos estudar uma importante técnica de transformar séries infinitas em integrais no plano complexo, que será fundamental para tratar as somas de Matsubara durante todo o trabalho.

Considere $f(z)$ uma função analítica (sem polos) sobre o eixo imaginário. Sabemos que a função $\operatorname{coth}(\beta z / 2)$ possui polos simples em $z=2 n \pi i / \beta$, de sorte que a função $\operatorname{coth}(\beta z / 2) f(z)$ também terá polos simples em $z=2 n \pi i / \beta$. Observando os contornos de integração na figura C.1, vamos avaliar a seguinte integral

$$
I=\oint_{C} d z \operatorname{coth}(\beta z / 2) f(z)
$$

Usando o teorema do resíduo podemos calcular $I$ como

$$
\begin{aligned}
I & =\oint_{\cup_{i} C_{i}} d z \operatorname{coth}(\beta z / 2) f(z)=2 \pi i \sum_{n=-\infty}^{\infty} \operatorname{Res}_{z_{0}=2 \pi n i / \beta} \operatorname{coth}\left(\beta z_{0} / 2\right) f\left(z_{0}\right) \\
& =2 \pi i \sum_{n=-\infty}^{\infty} \lim _{z_{0} \rightarrow 2 \pi n i / \beta}\left(z_{0}-2 n \pi i / \beta\right) \operatorname{coth}\left(\beta z_{0} / 2\right) f\left(z_{0}\right) \\
& =2 \pi i \sum_{n=-\infty}^{\infty} \lim _{z_{0} \rightarrow 2 n \pi i / \beta} f\left(z_{0}\right)\left(z_{0}-2 n \pi i / \beta\right) \frac{e^{\beta z_{0}}+1}{e^{\beta z_{0}}-1} \\
& =2 \pi i \sum_{n=-\infty}^{\infty} \lim _{z_{0} \rightarrow 2 n \pi i / \beta} f\left(z_{0}\right)\left(z_{0}-2 n \pi i / \beta\right) \\
& \times \frac{2+\left(\beta z_{0}-2 n \pi i\right)+\frac{\left(\beta z_{0}-2 n \pi i\right)^{2}}{2 !}+\ldots}{\left(\beta z_{0}-2 n \pi i\right)+\frac{\left(\beta z_{0}-2 n \pi i\right)^{2}}{2 !}+\ldots} \\
& =4 \pi i \sum_{n=-\infty}^{\infty} \frac{1}{\beta} f(2 n \pi i / \beta) .
\end{aligned}
$$

Assim, C.1 juntamente com C.2 nos permitem escrever as somas de Matsubara para bósons na forma

$$
\sum_{n=-\infty}^{\infty} \frac{1}{\beta} f(2 n \pi i / \beta)=\frac{1}{4 \pi i} I .
$$



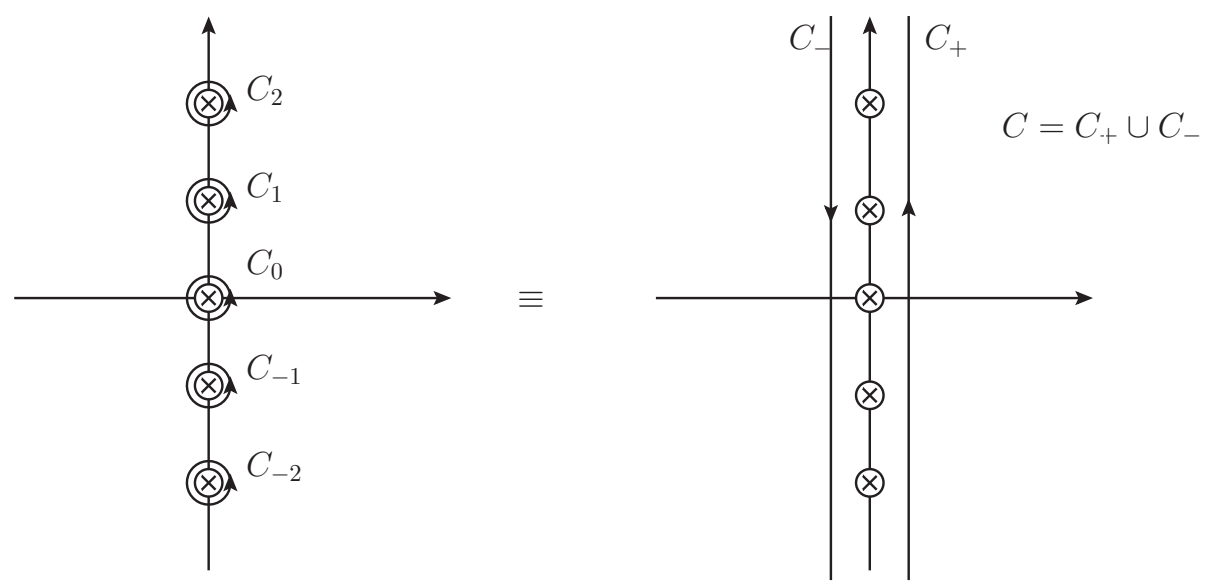

Figura C.1: Contornos de integração no plano complexo. Os círculos marcados com x representam os polos da função $\operatorname{coth}(\beta z / 2) f(z)$

Podemos escrever $I$ de maneira mais conveniente

$$
I=\int_{-i \infty+\epsilon}^{i \infty+\epsilon} d z \operatorname{coth}(\beta z / 2) f(z)+\int_{i \infty-\epsilon}^{-i \infty-\epsilon} d z \operatorname{coth}(\beta z / 2) f(z),
$$

e fazendo a mudança de variáveis $z \rightarrow-z$ na segunda integral e substituindo em (C.3) obtemos

$$
\sum_{n=-\infty}^{\infty} \frac{1}{\beta} f(2 n \pi i / \beta)=\int_{-i \infty+\epsilon}^{i \infty+\epsilon} \frac{d z}{2 \pi i} \operatorname{coth}(\beta z / 2)\left[\frac{f(z)+f(-z)}{2}\right] .
$$

A função coth pode ser rearranjada como

$$
\operatorname{coth}(\beta z / 2)=\frac{e^{\beta z}+1}{e^{\beta z}-1}=1+\frac{2}{e^{\beta z}-1}=1+2 N_{B}(z)
$$

e substituindo em (C.5) temos

$$
\begin{aligned}
\sum_{n=-\infty}^{\infty} \frac{1}{\beta} f(2 n \pi i / \beta) & =\int_{-i \infty+\epsilon}^{i \infty+\epsilon} \frac{d z}{2 \pi i}[f(z)+f(-z)] N_{B}(z) \\
& +\frac{1}{2 \pi i} \int_{-i \infty}^{i \infty} \frac{d z}{2 \pi i}\left[\frac{f(z)+f(-z)}{2}\right]
\end{aligned}
$$

onde

$$
N_{B}(z)=\frac{1}{e^{\beta z}-1}
$$

é a distribuição de Bose-Einstein. O segundo termo de (C.7) é o termo de temperatura zero da integral, enquanto o primeiro termo, que é dependente da temperatura, é proporcional a distribuição de Bose-Einstein. A relação algébrica (C.6) [assim como a (C.15)] mostra porque a escolha da função coth (tanh) é conveniente para fazer a separação da parte 


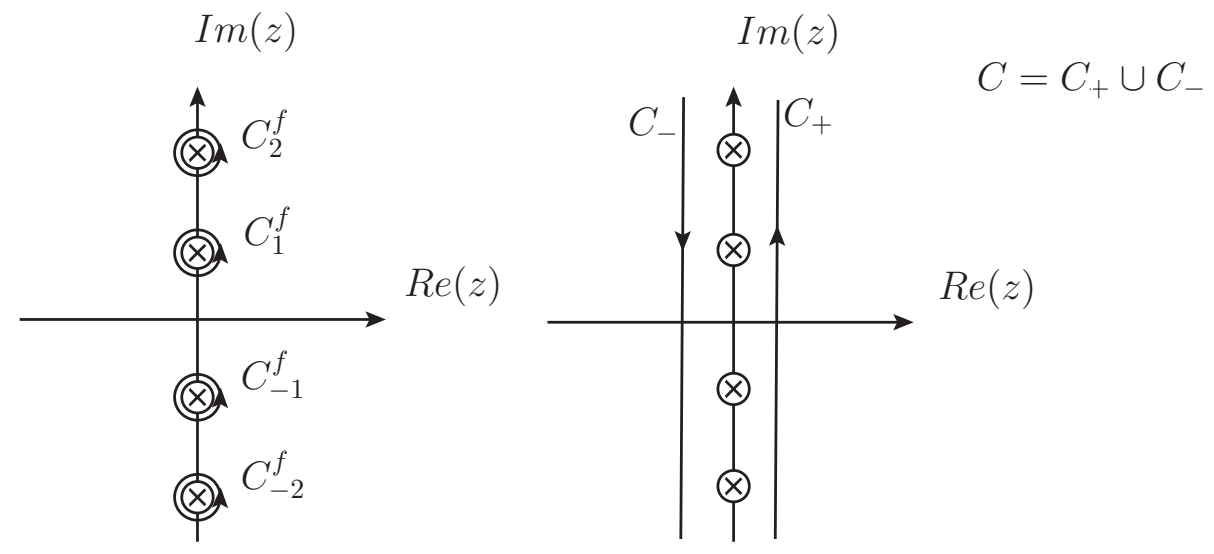

Figura C.2: Contornos de integração para frequência ímpares.

independente da temperatura.

No caso fermiônico a integração funcional é realizada sobre modos anti-periódicos do campo (veja a seção 2.2.3), de forma que os campos fermiônicos admitem uma expansão de Fourier do tipo

$$
\psi\left(\vec{x}, \tau_{x}\right)=\frac{1}{\sqrt{V}} \sum_{n, \vec{p}} e^{i\left(\vec{p} \cdot \vec{x}+\omega_{n}^{F} \tau_{x}\right)} \tilde{\psi}_{n}(\vec{p}),
$$

onde

$$
\omega_{n}^{F}=(2 n+1) \pi T
$$

é a frequência de Matsubara do férmion, que garante a anti-periodicidade do campo $\psi$. Note que o fator $V^{-1 / 2}$ é inserido de modo a conter toda a dimensão do lado direito de (C.9.

A equação C.10 juntamente com C.9 nos mostra que no caso fermiônico as somas de Matsubara são da forma

$$
\sum_{n} f((2 n+1) \pi T)
$$

De maneira análoga ao caso bosônico, vamos avaliar a integral

$$
I_{F}=\oint_{C} \tanh (\beta z / 2) f(z),
$$

onde $f(z)$, por hipótese, não tem polos sobre o eixo imaginário. Usando o teorema do 
resíduo teremos

$$
\begin{aligned}
I_{F} & =\oint_{\cup_{i} C_{i}^{f}} d z \tanh (\beta z / 2) f(z)=2 \pi i \sum_{n=-\infty}^{\infty} \operatorname{Res}_{z_{0}=(2 n+1) \pi i / \beta} \tanh \left(\beta z_{0} / 2\right) f\left(z_{0}\right) \\
& =2 \pi i \sum_{n=-\infty}^{\infty} \lim _{z_{0} \rightarrow(2 n+1) \pi i / \beta}\left(z_{0}-(2 n+1) \pi i / \beta\right) \tanh \left(\beta z_{0} / 2\right) f\left(z_{0}\right) \\
& =2 \pi i \sum_{n=-\infty}^{\infty} \lim _{z_{0} \rightarrow 2 n \pi i / \beta} f\left(z_{0}\right)\left(z_{0}-(2 n+1) \pi i / \beta\right) \frac{e^{\beta z_{0}}-1}{e^{\beta z_{0}}+1} \\
& =2 \pi i \sum_{n=-\infty}^{\infty} \lim _{z_{0} \rightarrow 2 n \pi i / \beta} f\left(z_{0}\right)\left(z_{0}-(2 n+1) \pi i / \beta\right) \frac{-e^{\beta z_{0}-(2 n+1) \pi i}-1}{-e^{\beta z_{0}-(2 n+1) \pi i}+1} \\
& =2 \pi i \sum_{n=-\infty}^{\infty} \lim _{z_{0} \rightarrow(2 n+1) \pi i / \beta} f\left(z_{0}\right)\left(z_{0}-(2 n+1) \pi i / \beta\right) \\
& \times \frac{2+\left(\beta z_{0}-(2 n+1) \pi i\right)+\frac{\left(\beta z_{0}-(2 n+1) \pi i\right)^{2}}{2 !}+\ldots}{\left(\beta z_{0}-(2 n+1) \pi i\right)+\frac{\left(\beta z_{0}-(2 n+1) \pi i\right)^{2}}{2 !}+\ldots} \\
& =4 \pi i \sum_{n=-\infty}^{\infty} \frac{1}{\beta} f((2 n+1) \pi i / \beta) .
\end{aligned}
$$

Logo, usando C.13 juntamente com C.12 podemos escrever uma soma de Matsubara sobre frequências ímpares como

$$
\sum_{n=-\infty}^{\infty} \frac{1}{\beta} f((2 n+1) \pi i / \beta)=\frac{1}{4 \pi i} I^{F} .
$$

A função $\tanh (\beta z / 2)$ pode ser decomposta como

$$
\tanh (\beta z / 2)=\frac{e^{\beta z}-1}{e^{\beta z}+1}=1-\frac{2}{e^{\beta z}+1}=1-2 N_{F}(z)
$$

de forma que decompomos C.14 como

$$
\begin{aligned}
\sum_{n=-\infty}^{\infty} \frac{1}{\beta} f((2 n+1) \pi i / \beta) & =-\int_{-i \infty+\epsilon}^{i \infty+\epsilon} \frac{d z}{2 \pi i}[f(z)+f(-z)] N_{F}(z) \\
& +\int_{-i \infty}^{i \infty} \frac{d z}{2 \pi i}\left[\frac{f(z)+f(-z)}{2}\right]
\end{aligned}
$$

onde $N_{F}(z)$ é a distribuição de Fermi-Dirac, que é escrita como

$$
N_{F}(z)=\frac{1}{e^{\beta z}+1}
$$


De modo geral, podemos escrever C.7 e C.16 como:

$$
\begin{aligned}
\sum_{n=-\infty}^{\infty} \frac{1}{\beta} f\left(\omega_{n}^{B / F}\right) & = \pm \frac{1}{2 \pi i} \int_{-i \infty+\epsilon}^{i \infty+\epsilon} \frac{d z}{2 \pi i}[f(z)+f(-z)] N_{B / F}(z) \\
& +\frac{1}{2 \pi i} \int_{-i \infty}^{i \infty} \frac{d z}{2 \pi i}\left[\frac{f(z)+f(-z)}{2}\right],
\end{aligned}
$$

onde $\omega_{n}^{B}=2 n \pi i$ e $\omega_{n}^{F}=(2 n+1) \pi i$ são as frequências de Matsubara bosônica e fermiônica, respectivamente, e $N_{B / F}$ são as distribuições de Bose-Einstein (C.8) e Fermi-Dirac (C.17).

Na seção 2.2.2 aplicamos este método para somar uma série do tipo

$$
\sum_{n} f\left(\omega_{n}^{p / i}\right)
$$

com

$$
\begin{aligned}
& \omega_{n}^{p}=2 n \pi \\
& \omega_{n}^{i}=(2 n+1) \pi .
\end{aligned}
$$

Lá a função $f\left(\omega_{n}^{p / i}\right)$ tem polos sobre o eixo imaginário mas não tem polos sobre eixo real. Neste caso podemos fazer um cálculo totalmente análogo usando as funções $\cot ^{ \pm 1}(z / 2)$, tendo em vista que estas funções possuem polos nos pontos $\omega_{n}^{p / i}$ respectivamente. Assim, usando o teorema do resíduo, calculamos C.19 como

$$
\sum_{n=-\infty}^{\infty} f\left(\omega_{n}^{p / i}\right)=\frac{1}{4 \pi}\left(\int_{-\infty-i \epsilon}^{\infty-i \epsilon} d z+\int_{\infty+i \epsilon}^{-\infty+i \epsilon} d z\right) f(z) \cot ^{ \pm 1}(z / 2) .
$$

Assim vemos que o método apresentado aqui é um poderoso método de somar diversos tipos de séries infinitas. 


\section{APÊNDICE D - Integral escalar para a função de dois pontos}

Neste apêndice vamos tratar a integral

$$
\mathcal{I}=\int \frac{d \Omega_{d-1}}{\Omega_{d-1}} \frac{1}{1-z \hat{Q} \cdot \hat{k}}
$$

que aparece frequentemente no cálculo da parte térmica da função de dois pontos em vários modelos em teorias de campos. Esta integral será representada em termos da função hipergeométrica de Gauss

Em um espaço $(d-1)$-dimensional a integração sobre os ângulos possuí a forma explicita dada por

$$
\begin{aligned}
\int d \Omega_{d-1} & =\int_{0}^{2 \pi} d \phi \prod_{j=1}^{d-3}\left[\int_{0}^{\pi} d \theta_{j}\left(\sin \theta_{j}\right)^{j}\right] \\
& =\int d \Omega_{d-2} \int_{0}^{\pi} d \theta_{d-3}\left(\sin \theta_{d-3}\right)^{d-3}
\end{aligned}
$$

onde temos 1 ângulo azimutal e $d-3$ ângulos polares. Podemos escolher o sistema de coordenadas de maneira que $\theta_{d-3}$ é o ângulo entre $\vec{Q}$ e $\vec{k}$, sem perda de generalidade. 
Desta forma temos

$$
\begin{aligned}
\mathcal{I} & =\frac{\Omega_{d-2}}{\Omega_{d-1}} \int_{0}^{\pi} d \theta \frac{(\sin \theta)^{(d-3)}}{1-z \cos \theta} \\
& =\frac{\Omega_{d-2}}{\Omega_{d-1}} \int_{-1}^{1} d x \frac{\left(1-x^{2}\right)^{(d-4) / 2}}{1-z x} \\
& =\frac{\Omega_{d-2}}{\Omega_{d-1}} \int_{-1}^{1} d x \frac{1}{2}\left[\frac{\left(1-x^{2}\right)^{(d-4) / 2}}{1-z x}+\frac{\left(1-x^{2}\right)^{(d-4) / 2}}{1+z x}\right] \\
& =\frac{\Omega_{d-2}}{\Omega_{d-1}} \int_{-1}^{1} d x \frac{\left(1-x^{2}\right)^{(d-4) / 2}}{1-z^{2} x^{2}} \\
& =\frac{\Omega_{d-2}}{\Omega_{d-1}} \int_{0}^{1} d x \frac{\left(1-x^{2}\right)^{(d-4) / 2}}{1-z^{2} x^{2}} \\
& =\frac{\Omega_{d-2}}{\Omega_{d-1}} \int_{0}^{1} d t \frac{(1-t)^{(d-4) / 2} t^{-1 / 2}}{1-z^{2} t} .
\end{aligned}
$$

Na ultima forma identificamos a representação integral da função hipergeométrica de Gauss

$$
{ }_{2} F_{1}(a, b ; c ; \xi)=\frac{\Gamma(c)}{\Gamma(c-b) \Gamma(b)} \int_{0}^{1} d t \frac{(1-t)^{c-b-1} t^{b-1}}{(1-\xi t)^{a}},
$$

de modo que teremos

$$
\mathcal{I}=\frac{\Omega_{d-2}}{\Omega_{d-1}} \frac{\Gamma(1 / 2) \Gamma\left(\frac{d-2}{2}\right)}{\Gamma\left(\frac{d-1}{2}\right)}{ }_{2} F_{1}\left(1, \frac{1}{2} ; \frac{d-1}{2} ; z^{2}\right)={ }_{2} F_{1}\left(1, \frac{1}{2} ; \frac{d-1}{2} ; \frac{\vec{k}^{2}}{k_{0}^{2}}\right)={ }_{2} F_{1}\left(\frac{1}{2}, 1 ; \frac{d-1}{2} ; \frac{\vec{k}^{2}}{k_{0}^{2}}\right) .
$$

No caso especial em que $d=4$, para $k_{0}>|\vec{k}|$, teremos [GR80]

$$
\begin{aligned}
\mathcal{I}_{d=4} & ={ }_{2} F_{1}\left(\frac{1}{2}, 1 ; \frac{3}{2} ; \frac{\vec{k}^{2}}{k_{0}^{2}}\right) \\
& =\frac{k_{0}}{2|\vec{k}|} \log \left(\frac{k_{0}+|\vec{k}|}{k_{0}-|\vec{k}|}\right) .
\end{aligned}
$$

Em certos momentos, será conveniente usar outra representação da função hipergeométrica usando a identidade

$$
{ }_{2} F_{1}(a, b, c, z)=(1-z)_{2}^{-a} F_{1}\left(a, c-b, c, \frac{z}{z-1}\right),
$$

que implica em

$$
\mathcal{I}=\frac{k_{0}^{2}}{k^{2}}{ }_{2} F_{1}\left(\frac{1}{2}, \frac{d-3}{2}, \frac{d-1}{2},-\frac{|\vec{k}|^{2}}{k^{2}}\right)
$$




\section{$A P \hat{E} N D I C E E-$ Limite de altas temperaturas das funções de Green estáticas}

Neste apêndice completaremos a dedução da identidade que relaciona as funções de Green estáticas e de momento externo nulo [BS12b] para a auto-energia em ordem de dois laços (veja seção 5.3). Consideraremos aqui os diagramas mostrados nas figuras E.1, E.2 e E.3.

\section{E.1 Terceira topologia}

Vamos começar com a topologia mostrada na figura E.1 que no limte estático fornece

$$
\Pi_{e}^{C}=\oint_{C} \frac{d p_{0}}{2 \pi i} \oint_{C} \frac{d q_{0}}{2 \pi i} \frac{f^{C}\left(p_{0}, q_{0}\right)}{\left(p_{0}^{2}-w^{2}\right)\left[\left(p_{0}-q_{0}\right)^{2}-z^{2}\right]\left(q_{0}^{2}-y^{2}\right)}
$$

onde estamos usando a notação $w=|\vec{p}+\vec{k}|, y=|\vec{q}|$ e $z=|\vec{p}-\vec{q}|$.

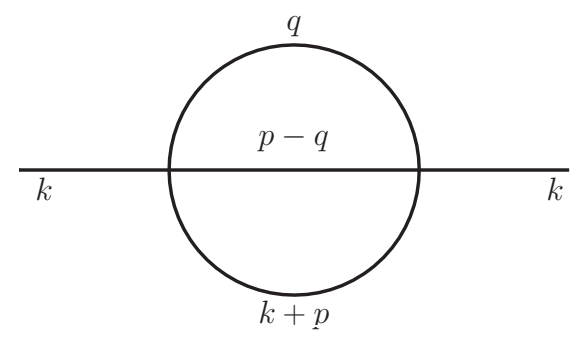

Figura E.1: Terceira topologia para a auto-energia em ordem dois laços. 
Fazendo a integração em $p_{0}$, com a ajuda do teorema dos resíduos, obtemos

$$
\begin{aligned}
\Pi_{e}^{C} & =-\oint_{C} \frac{d q_{0}}{2 \pi i}\left\{\left[\frac{f^{C}\left(w, q_{0}\right)}{2 w\left[\left(w-q_{0}\right)^{2}-z^{2}\right]\left(q_{0}^{2}-y^{2}\right)}+\frac{f^{C}\left(q_{0}+z, q_{0}\right)}{2 z\left[\left(q_{0}+z\right)^{2}-w^{2}\right]\left(q_{0}^{2}-y^{2}\right)}\right]\right. \\
& +[w, z \rightarrow-w,-z]\} .
\end{aligned}
$$

Similarmente, a integração em $q_{0}$ fornece

$$
\begin{aligned}
\Pi_{e}^{C} & =\left\{\left[\frac{f^{C}(w, y)}{4 w y\left[(w-y)^{2}-z^{2}\right]}+\frac{f^{C}(y+z, y)}{4 z y\left[(y+z)^{2}-w^{2}\right]}+(y \rightarrow-y)\right]\right. \\
& \left.+\left[\frac{f^{C}(w, w+z)}{4 w z\left[(w+z)^{2}-y^{2}\right]}+(z \rightarrow-z)\right]+\left[\frac{f^{C}(w, w-z)}{4 z w\left[(w-z)^{2}-y^{2}\right]}+(w \rightarrow-w)\right]\right\} \\
& +\{w, z \rightarrow-w-z\} .
\end{aligned}
$$

No limite de altas temperaturas vamos considerar $w=x+\epsilon$, com $x=|\vec{p}|$, de modo que o termo dominante será

$$
\begin{aligned}
\Pi_{e}^{C} & \simeq\left\{\left[\frac{f^{C}(x, y)}{4 x y\left[(x-y)^{2}-z^{2}\right]}+\frac{f^{C}(y+z, y)}{4 z y\left[(y+z)^{2}-x^{2}\right]}+(y \rightarrow-y)\right]\right. \\
& \left.+\left[\frac{f^{C}(x, x+z)}{4 x z\left[(x+z)^{2}-y^{2}\right]}+(z \rightarrow-z)\right]+\left[\frac{f^{C}(x, x-z)}{4 z x\left[(x-z)^{2}-y^{2}\right]}+(x \rightarrow-x)\right]\right\} \\
& +\{x, z \rightarrow-x-z\} \\
& =\oint_{C} \frac{d p_{0}}{2 \pi i} \oint_{C} \frac{d q_{0}}{2 \pi i} \frac{f^{C}\left(p_{0}, q_{0}\right)}{\left(p_{0}^{2}-x^{2}\right)\left[\left(p_{0}-q_{0}\right)^{2}-z^{2}\right]\left(q_{0}^{2}-y^{2}\right)} \\
& =\Pi_{0}^{C},
\end{aligned}
$$

onde identificamos a amplitude no limite de momento externo nulo

$$
\Pi_{0}^{C}=\oint_{C} \frac{d p_{0}}{2 \pi i} \oint_{C} \frac{d q_{0}}{2 \pi i} \frac{f^{C}\left(p_{0}, q_{0}\right)}{\left(p_{0}^{2}-x^{2}\right)\left[\left(p_{0}-q_{0}\right)^{2}-z^{2}\right]\left(q_{0}^{2}-y^{2}\right)}
$$

que estabelece a identidade entre a função de Green estática e de momento externo nulo, no limite de altas temperaturas.

\section{E.2 Quarta topologia}

Vamos agora considerar a topologia mostrada na figura E.2 que, no limite estático, possui a seguinte forma

$$
\Pi_{e}^{D}=\oint_{C} \frac{d p_{0}}{2 \pi i} \oint_{C} \frac{d q_{0}}{2 \pi i} \frac{f^{D}\left(p_{0}, q_{0}\right)}{\left(p_{0}^{2}-x^{2}\right)\left(p_{0}^{2}-w^{2}\right)\left[\left(p_{0}-q_{0}\right)^{2}-z^{2}\right]\left(q_{0}^{2}-y^{2}\right)},
$$

onde estamos usando a notação $x=|\vec{p}|, y=|\vec{q}|, w=|\vec{p}+\vec{k}|$ and $z=|\vec{p}-\vec{q}|$. 


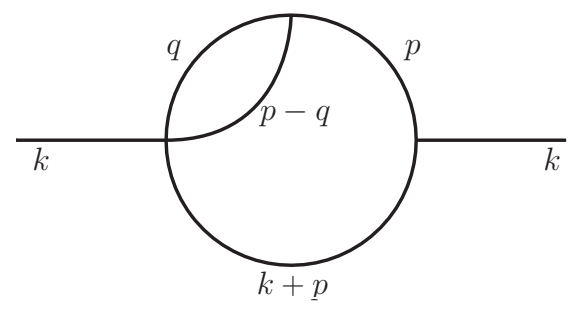

Figura E.2: Quarta topologia para a auto-energia em ordem dois laços.

Como feito antes, vamos usar o teorema dos resíduos para fazer a integração em $p_{0} \mathrm{e}$ em $q_{0}$, obtendo

$$
\begin{aligned}
\Pi_{e}^{D} & =\left\{\left[\frac{f^{D}(x, y)}{4 x y\left(x^{2}-w^{2}\right)\left[(x-y)^{2}-z^{2}\right]}+\frac{f^{D}(w, y)}{4 w y\left(w^{2}-x^{2}\right)\left[(w-y)^{2}-w^{2}\right]}\right.\right. \\
& \left.+\frac{f^{D}(y+z, y)}{4 z y\left[(y+z)^{2}-x^{2}\right]\left[(y+z)^{2}-w^{2}\right]}+(y \rightarrow-y)\right] \\
& +\left[\frac{f^{D}(x, x+z)}{4 z x\left(x^{2}-w^{2}\right)\left[(x+z)^{2}-y^{2}\right]}+\frac{f^{D}(w, w+z)}{4 z w\left(w^{2}-x^{2}\right)\left[(w+z)^{2}-y^{2}\right]}+(z \rightarrow-z)\right] \\
& +\left[\frac{f^{D}(x, x-z)}{4 x z\left(x^{2}-w^{2}\right)\left[(x-z)^{2}-y^{2}\right]}+(x \rightarrow-x)\right] \\
& \left.+\left[\frac{f^{D}(w, w-z)}{4 w z\left(w^{2}-x^{2}\right)\left[(w-z)^{2}-y^{2}\right]}+(w \rightarrow-w)\right]\right\}+\{(x, w, z) \rightarrow(-x,-w,-z)\} .
\end{aligned}
$$

No limite de altas temperaturas a expressão 5.23 possui a forma

$$
\begin{aligned}
\Pi_{e}^{D} & \simeq\left\{\left[\frac{1}{2 x} \frac{\partial}{\partial x} \frac{f^{D}(x, y)}{4 x y\left[(x-y)^{2}-z^{2}\right]}+\frac{f^{D}(y+z, y)}{4 z y\left[(y+z)^{2}-x^{2}\right]^{2}}+(y \rightarrow-y)\right]\right. \\
& +\left[\frac{1}{2 x} \frac{\partial}{\partial x} \frac{f^{D}(x, x+z)}{4 z x\left[(x+z)^{2}-y^{2}\right]}+(z \rightarrow-z)\right] \\
& \left.+\left[\frac{1}{2 x} \frac{\partial}{\partial x} \frac{f^{D}(x, x-z)}{4 x z\left[(x-z)^{2}-y^{2}\right]}+(x \rightarrow-x)\right]\right\}+\{(x, z) \rightarrow(-x,-z)\} .
\end{aligned}
$$

Por outro lado, a amplitude no limite de momento externo nulo fornece

$$
\Pi_{0}^{D}=\oint_{C} \frac{d p_{0}}{2 \pi i} \oint_{C} \frac{d q_{0}}{2 \pi i} \frac{f^{D}\left(p_{0}, q_{0}\right)}{\left(p_{0}^{2}-x^{2}\right)^{2}\left[\left(p_{0}-q_{0}\right)^{2}-z^{2}\right]\left(q_{0}^{2}-y^{2}\right)} .
$$

Usando o teorema dos resíduos para integração em $p_{0}$ teremos

$$
\begin{aligned}
\Pi_{0}^{D} & =-\oint_{C} \frac{d q_{0}}{2 \pi i}\left\{\left[\frac{f^{D}\left(q_{0}+z, q_{0}\right)}{2 z\left[\left(q_{0}+z\right)^{2}-x^{2}\right]^{2}\left(q_{0}^{2}-y^{2}\right)}+\frac{1}{2 x} \frac{\partial}{\partial x} \frac{f^{D}\left(x, q_{0}\right)}{2 x\left[\left(x-q_{0}\right)^{2}-z^{2}\right]\left(q_{0}^{2}-y^{2}\right)}\right]\right. \\
& +[(x, z) \rightarrow(-x,-z)]\} .
\end{aligned}
$$




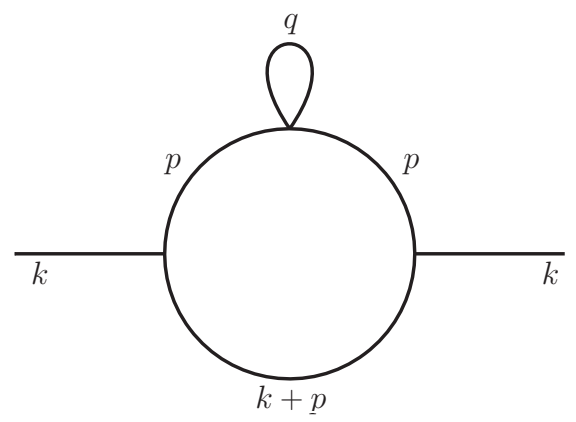

Figura E.3: Quinta topologia para a auto-energia em ordem dois laços.

De maneira análoga, integrando em $q_{0}$ obtemos

$$
\begin{aligned}
\Pi_{0}^{D} & =\left\{\left[\frac{f^{D}(y+z, y)}{4 z y\left[(y+z)^{2}-x^{2}\right]^{2}} \frac{1}{2 x} \frac{\partial}{\partial x} \frac{f^{D}(x, y)}{4 x y\left[(x-y)^{2}-z^{2}\right]}+(y \rightarrow-y)\right]\right. \\
& +\left[\frac{1}{2 x} \frac{\partial}{\partial x} \frac{f^{D}(x, x+z)}{4 x z\left[(x+z)^{2}-y^{2}\right]}+(z \rightarrow-z)\right] \\
& \left.+\left[\frac{1}{2 x} \frac{\partial}{\partial x} \frac{f^{D}(x, x-z)}{4 z x\left[(x-z)^{2}-y^{2}\right]}+(x \rightarrow-x)\right]\right\}+\{(x, z) \rightarrow(-x,-z)\} .
\end{aligned}
$$

Portanto, comparando o limite estático E.8) com o limite de momento esterno nulo (E.11) vemos que eles são idênticos no limite de altas temperaturas para amplitude mostrada na figura E.2.

\section{E.3 Quinta topologia}

A quinta topologia para a auto-energia em ordem dois laços está mostrada na figura E.3. No limite estático ela se reduz à

$$
\Pi_{e}^{E}=\oint_{C} \frac{d p_{0}}{2 \pi i} \oint_{C} \frac{d q_{0}}{2 \pi i} \frac{f^{E}\left(p_{0}, q_{0}\right)}{\left(p_{0}^{2}-x^{2}\right)^{2}\left(p_{0}^{2}-w^{2}\right)\left(q_{0}^{2}-y^{2}\right)},
$$

$\operatorname{com} x=|\vec{p}|, y=|\vec{q}|$ e $w=|\vec{p}+\vec{k}|$. Fazendo a integração em $p_{0}$ com a ajuda do teorema dos resíduos obtemos

$$
\begin{aligned}
\Pi_{e}^{E} & =-\oint_{C} \frac{d q_{0}}{2 \pi i}\left\{\frac{f^{E}\left(w, q_{0}\right)}{2 w\left(w^{2}-x^{2}\right)^{2}\left(q_{0}^{2}-y^{2}\right)}+(w \rightarrow-w)\right. \\
& \left.+\frac{1}{2 x} \frac{\partial}{\partial x} \frac{f^{E}\left(x, q_{0}\right)}{2 x\left(x^{2}-w^{2}\right)\left(q_{0}^{2}-y^{2}\right)}+(x \rightarrow-x)\right\} .
\end{aligned}
$$

Nesta amplitude os laços não se sobrepõem de moo que não é necessário, para o nosso propósito, calcular a integral na variável $q_{0}$. 
No limite de altas temperaturas podemos usar a aproximação 5.73 e a identidade (5.75) que leva até

$$
\Pi_{e}^{E} \simeq-\oint_{C} \frac{d q_{0}}{2 \pi i}\left\{\frac{3}{16 x^{5}} \frac{f^{E}\left(x, q_{0}\right)}{\left(q_{0}^{2}-y^{2}\right)}-\frac{3}{16 x^{4}} \frac{\partial}{\partial x} \frac{f^{E}\left(x, q_{0}\right)}{\left(q_{0}^{2}-y^{2}\right)}+\frac{1}{16 x^{3}} \frac{\partial^{2}}{\partial x^{2}} \frac{f^{E}\left(x, q_{0}\right)}{\left(q_{0}^{2}-y^{2}\right)}+(x \rightarrow-x)\right\} .
$$

Quando todas as componente do momento externo se anulam, a amplitude se reduz à

$$
\Pi_{0}^{E}=\oint_{C} \frac{d p_{0}}{2 \pi i} \oint_{C} \frac{d q_{0}}{2 \pi i} \frac{f^{E}\left(p_{0}, q_{0}\right)}{\left(p_{0}^{2}-x^{2}\right)^{3}\left(q_{0}^{2}-y^{2}\right)}
$$

Usando o teorema dos resíduos para fazer a integral em $p_{0}$, obtemos

$$
\Pi_{0}^{E}=-\oint_{C} \frac{d q_{0}}{2 \pi i}\left[\frac{1}{2} \lim _{p_{0}=x} \frac{\partial^{2}}{\partial p_{0}^{2}} \frac{f^{E}\left(p_{0}, q_{0}\right)}{\left(p_{0}+x\right)^{3}\left(q_{0}^{2}-y^{2}\right)}+(x \rightarrow-x)\right] .
$$

Finalmente, computando o limite

$$
\Pi_{0}^{E}=-\oint_{C} \frac{d q_{0}}{2 \pi i}\left\{\frac{3}{16 x^{5}} \frac{f^{E}\left(x, q_{0}\right)}{\left(q_{0}^{2}-y^{2}\right)}-\frac{3}{16 x^{4}} \frac{\partial}{\partial x} \frac{f^{E}\left(x, q_{0}\right)}{\left(q_{0}^{2}-y^{2}\right)}+\frac{1}{16 x^{3}} \frac{\partial^{2}}{\partial x^{2}} \frac{f^{E}\left(x, q_{0}\right)}{\left(q_{0}^{2}-y^{2}\right)}+(x \rightarrow-x)\right\} .
$$

Portanto, o limite estático E.14 e o limite de momento externo nulo E.17) são idênticos no limite de altas temperaturas, também para a topologia mostrada na figura E.3. Isto mostra que quando os campos externos são estáticos, podemos obter o limite de altas temperaturas da auto-energia até ordem dois laços supondo que todas as componentes do momento externo são nulas. 


\section{APÊEDICE F - Obtenção da auto-energia estática do fóton a partir da ação efetiva}

Nesta seção mostraremos que a expressão da lagrangiana efetiva do campo de Maxwell em ordem de um laço (6.4) e dois laços 6.20), de fato geram corretamente as funções de Green 1PI de dois pontos.

\section{F.1 Auto-energia em ordem de um laço}

Em ordem de um laço a auto-energia, representada na figura F.1, possui a forma que segue

$$
\begin{aligned}
\Pi^{\mu \nu}(k) & =e^{2} T \sum_{n} \int \frac{d^{d-1} p}{(2 \pi)^{d-1}} \operatorname{tr}\left[\gamma^{\mu} \frac{1}{\not p} \gamma^{\nu} \frac{1}{\not k+\not p}\right] \\
& =e^{2} \operatorname{tr}\left[\gamma^{\mu} \gamma^{\alpha} \gamma^{\nu} \gamma^{\lambda}\right] T \sum_{n} \int \frac{d^{d-1} p}{(2 \pi)^{d-1}} \frac{p_{\alpha}(k+p)_{\lambda}}{p^{2}(k+p)^{2}}
\end{aligned}
$$

Os termos no numerador que são proporcionais ao momento externo $k$ são subdominantes no limite de altas temperaturas [Bel96], e a contribuição dominante possui a forma

$$
\Pi^{\mu \nu}(k) \simeq e^{2} \operatorname{tr}\left[\gamma^{\mu} \gamma^{\alpha} \gamma^{\nu} \gamma^{\lambda}\right] T \sum_{n} \int \frac{d^{d-1} p}{(2 \pi)^{d-1}} \frac{p_{\alpha} p_{\lambda}}{p^{2}(k+p)^{2}},
$$

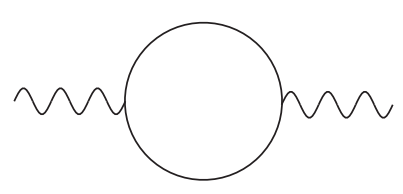

Figura F.1: One loop self energy. 
e no limite estático escrevemos

$$
\Pi^{\mu \nu}\left(k_{0}=0\right) \simeq e^{2} \operatorname{tr}\left[\gamma^{\mu} \gamma^{\alpha} \gamma^{\nu} \gamma^{\lambda}\right] T \sum_{n} \int \frac{d^{d-1} p}{(2 \pi)^{d-1}} \frac{p_{\alpha} p_{\lambda}}{\left(-p_{0}^{2}+|\vec{p}|^{2}\right)\left(-p_{0}^{2}+|\vec{k}+\vec{p}|^{2}\right)}
$$

Portanto, usando usando os argumentos de seção 5.2.1, teremos a seguinte aproximação na região dos HTL

$$
\begin{aligned}
\Pi^{\mu \nu}\left(k_{0}=0\right) & \simeq e^{2} \operatorname{tr}\left[\gamma^{\mu} \gamma^{\alpha} \gamma^{\nu} \gamma^{\lambda}\right] T \sum_{n} \int \frac{d^{d-1} p}{(2 \pi)^{d-1}} \frac{p_{\alpha} p_{\lambda}}{\left(-p_{0}^{2}+|\vec{p}|^{2}\right)^{2}} \\
& =e^{2} T \sum_{n} \int \frac{d^{d-1} p}{(2 \pi)^{d-1}} \operatorname{tr}\left[\gamma^{\mu} \frac{1}{\not p} \gamma^{\nu} \frac{1}{\not p}\right]
\end{aligned}
$$

Vamos verificar que esta amplitude é gerada pela definição da ação efetiva (6.4)

$$
\begin{aligned}
\mathcal{L}_{\text {eff }}^{(1)} & =\frac{1}{\beta V} \log \operatorname{Det} \beta i \gamma^{\mu}\left(p_{\mu}-e A_{\mu}^{C}\right) \\
& =T \sum_{n} \int \frac{d^{d-1} p}{(2 \pi)^{d-1}} \operatorname{tr} \log \left[\beta i \gamma^{\mu}\left(p_{\mu}-e A_{\mu}^{C}\right)\right] .
\end{aligned}
$$

Definindo $\pi_{p}^{\mu}=p_{\mu}-e A_{\mu}^{C}$, vamos calcular a derivada segunda de

$$
\frac{\delta^{2}}{\delta A_{\nu}^{C} \delta A_{\mu}^{C}} \operatorname{tr} \log \left(\beta i \pi_{p}\right)
$$

para ver como a derivada segunda da lagrangiana efetiva $\mathrm{F} .5$ está relacionada com a auto-energia. Usando a definição do logaritmo de uma matriz e a ciclicidade do traço, a primeira derivada pode ser calculada como

$$
\begin{aligned}
\frac{\delta}{\delta A_{\mu}^{C}} \operatorname{tr} \log \left(\beta i \pitchfork_{p}\right) & =\operatorname{tr} \frac{\delta}{\delta A_{\mu}} \sum_{l=1}^{\infty} \frac{(-1)^{l-1}}{l}\left(\beta i \pitchfork_{p}-1\right)^{l} \\
& =\operatorname{tr} \sum_{l=1}^{\infty} \frac{(-1)^{l-1}}{l} \sum_{j=0}^{l-1}\left(\beta i \pitchfork_{p}-1\right)^{j}\left(-\beta i e \gamma^{\mu}\right)\left(\beta i \pitchfork_{p}-1\right)^{l-1-j} \\
& =\operatorname{tr} \sum_{l=1}^{\infty}(-1)^{l-1}\left(-\beta i e \gamma^{\mu}\right)\left(\beta i \oiint_{p}-1\right)^{l-1} \\
& =\operatorname{tr}\left(-e \gamma^{\mu} \frac{1}{\not_{p}}\right) .
\end{aligned}
$$


Para obter derivada segunda, usaremos a álgebra das matrizes de Dirac para notar que

$$
\begin{aligned}
\frac{\delta}{\delta A_{\nu}^{C}} \frac{1}{\not \pi_{p}} & =\frac{\delta}{\delta A_{\nu}^{C}} \frac{\not_{p}}{\left(\pi_{p}\right)^{\alpha}\left(\pi_{p}\right)_{\alpha}}=-e \gamma^{\nu} \frac{1}{\left(\pi_{p}\right)^{\alpha}\left(\pi^{p}\right)_{\alpha}}-2 e \pi_{p}^{\nu} \frac{(-1)}{\left[\left(\pi_{p}\right)^{\alpha}\left(\pi^{p}\right)_{\alpha}\right]^{2}} \pi / p \\
& =-e \gamma^{\nu} \frac{1}{\left(\pi_{p}\right)^{\alpha}\left(\pi_{p}\right)_{\alpha}}+\frac{2 e\left(\pi_{p}\right)^{\nu}}{\left(\pi_{p}\right)^{\alpha}\left(\pi_{p}\right)_{\alpha}} \frac{1}{\pi_{p}},
\end{aligned}
$$

que pode ser reescrita, usando a álgebra de Clifford, como

$$
\begin{aligned}
\frac{1}{\not_{p}} e \gamma^{\nu} \frac{1}{\not_{p}} & =\frac{\left(\pi_{p}\right)_{\mu}}{\left(\pi_{p}\right)^{\alpha}\left(\pi_{p}\right)_{\alpha}} e \gamma^{\mu} \gamma^{\nu} \frac{1}{\pi_{p}} \\
& =e\left(-\gamma^{\nu} \gamma^{\mu}+2 \eta^{\mu \nu}\right) \frac{\left(\pi_{p}\right)_{\mu}}{\left(\pi_{p}\right)^{\alpha}\left(\pi_{p}\right)_{\alpha}} \frac{1}{\pi_{p}} \\
& =-e \gamma^{\nu} \frac{1}{\left(\pi_{p}\right)^{\alpha}\left(\pi_{p}\right)_{\alpha}}+\frac{2 e\left(\pi_{p}\right)^{\nu}}{\left(\pi_{p}\right)^{\alpha}\left(\pi_{p}\right)_{\alpha}} \frac{1}{\pi_{p}} \\
& =\frac{\delta}{\delta A_{\nu}^{C}} \frac{1}{\pi_{p}},
\end{aligned}
$$

e assim substituindo (F.9) e (F.7) em (F.6) teremos

$$
\frac{\delta^{2}}{\delta A_{\nu} \delta A_{\mu}} \operatorname{tr} \log \left(\beta \oiint_{p}\right)=-e^{2} \operatorname{tr}\left(\gamma^{\mu} \frac{1}{\mathbb{t}_{p}} \gamma^{\nu} \frac{1}{\not_{p}}\right) .
$$

Logo, substituindo (F.10) em (F.5) o resultado a segunda derivada está relacionada com a auto-energia estática (F.4) como

$$
\left.\frac{\delta^{2}}{\delta A_{\nu} \delta A_{\mu}} \mathcal{L}_{\text {eff }}^{(1)}\right|_{A^{C}=0}=-e^{2} T \sum_{n} \int \frac{d^{d-1} p}{(2 \pi)^{d-1}} \operatorname{tr}\left[\gamma^{\mu} \frac{1}{\not p} \gamma^{\nu} \frac{1}{\not p}\right] \simeq-\Pi_{\mu \nu}\left(k_{0}=0\right),
$$

no limite de altas temperaturas, que está em completo acordo com 4.111) e 6.18).

\section{F.2 Auto-energia em ordem dois laços}

Para a auto-energia em ordem de dois laços, teremos duas topologias mostradas na figura F.2. Para a primeira [figura F.2 (a)] topologia teremos

$$
\begin{aligned}
\Pi_{\mu \nu}^{(2 a)}(k) & =e^{4} T^{2} \sum_{n_{p} n_{q}} \int \frac{d^{d-1} p}{(2 \pi)^{d-1}} \frac{d^{d-1} q}{(2 \pi)^{d-1}} \operatorname{tr}\left(\gamma^{\alpha} \frac{1}{\not p} \gamma^{\mu} \frac{1}{\not p+\not k} \gamma_{\alpha} \frac{1}{q+\not k} \gamma^{\nu} \frac{1}{\not q}\right) \frac{1}{(p-q)^{2}} \\
& =e^{4} T^{2} \sum_{n_{p} n_{q}} \int \frac{d^{d-1} p}{(2 \pi)^{d-1}} \frac{d^{d-1} q}{(2 \pi)^{d-1}} \operatorname{tr}\left(\gamma^{\alpha} \gamma^{\lambda_{1}} \gamma^{\mu} \gamma^{\lambda_{2}} \gamma_{\alpha} \gamma^{\lambda_{3}} \gamma^{\nu} \gamma^{\lambda_{4}}\right) \\
& \times \frac{p_{\lambda_{1}}(p+k)_{\lambda_{2}}(q+k)_{\lambda_{3}} q_{\lambda_{4}}}{p^{2}(p+k)^{2} q^{2}(q+k)^{2}(p-q)^{2}} .
\end{aligned}
$$




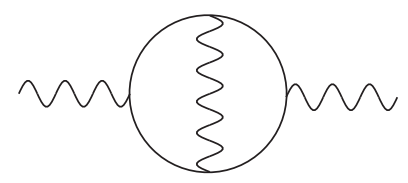

(a)

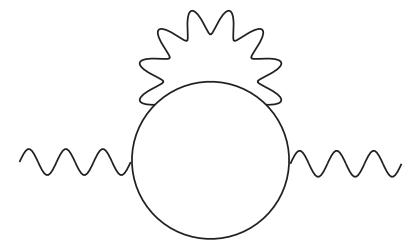

(b)

Figura F.2: Auto-energia em ordem dois laços.

Primeiramente, no limite de altas temperaturas podemos fazer $k=0$ no numerador [Bel96], obtendo

$$
\begin{aligned}
\Pi_{\mu \nu}^{(2 a)}(k) & \simeq e^{4} T^{2} \sum_{n_{p} n_{q}} \int \frac{d^{d-1} p}{(2 \pi)^{d-1}} \frac{d^{d-1} q}{(2 \pi)^{d-1}} \operatorname{tr}\left(\gamma^{\alpha} \gamma^{\lambda_{1}} \gamma^{\mu} \gamma^{\lambda_{2}} \gamma_{\alpha} \gamma^{\lambda_{3}} \gamma^{\nu} \gamma^{\lambda_{4}}\right) \\
& \times \frac{p_{\lambda_{1}} p_{\lambda_{2}} q_{\lambda_{3}} q_{\lambda_{4}}}{p^{2}(p+k)^{2} q^{2}(q+k)^{2}(p-q)^{2}}
\end{aligned}
$$

Esta topologia recai no caso tratado na seção 5.3.1. Assim, no limite de altas temperaturas, os argumentos que relacionam as funções de Green estáticas e de momento externo nulo podem ser aplicados aqui, de modo que teremos

$$
\begin{aligned}
\Pi_{\mu \nu}^{(2 a)}\left(k_{0}=0\right) & \simeq e^{4} T^{2} \sum_{n_{p} n_{q}} \int \frac{d^{d-1} p}{(2 \pi)^{d-1}} \frac{d^{d-1} q}{(2 \pi)^{d-1}} \operatorname{tr}\left(\gamma^{\alpha} \frac{1}{\not p} \gamma^{\mu} \frac{1}{\not p} \gamma_{\alpha} \frac{1}{\not q} \gamma^{\nu} \frac{1}{\not q}\right) \frac{1}{(p-q)^{2}} \\
& =\Pi_{\mu \nu}^{(2 a)}(k=0) .
\end{aligned}
$$

Para a segunda topologia [figura F.2(b)], os mesmos argumentos também se aplicam (veja seção 5.3.2, de modo que teremos

$$
\begin{aligned}
\Pi_{\mu \nu}^{(2 b)}\left(k_{0}=0\right) & \simeq e^{4} T^{2} \sum_{n_{p} n_{q}} \int \frac{d^{d-1} p}{(2 \pi)^{d-1}} \frac{d^{d-1} q}{(2 \pi)^{d-1}} \operatorname{tr}\left(\gamma^{\alpha} \frac{1}{\not p} \gamma^{\mu} \frac{1}{\not p} \gamma^{\nu} \frac{1}{\not p} \gamma_{\alpha} \frac{1}{\not q}\right) \frac{1}{(p-q)^{2}}+(\mu \leftrightarrow \nu) \\
& =\Pi_{\mu \nu}^{(2 b)}(k=0) .
\end{aligned}
$$

Agora vamos mostrar que a contribuição em ordem de dois laços para a lagrangiana 
efetiva estática 6.20

$$
\mathcal{L}_{\text {eff }}^{(2)}=-\frac{e^{2} T^{2}}{2} \sum_{n_{p} n_{q}} \int \frac{d^{d-1} p}{(2 \pi)^{d-1}} \frac{d^{d-1} q}{(2 \pi)^{d-1}} \operatorname{tr}\left(\gamma^{\mu} \frac{1}{\pi_{p}} \gamma_{\mu} \frac{1}{\pi_{q}}\right) \frac{1}{(p-q)^{2}},
$$

de fato gera estes resultados.

O uso sucessivo de (F.9) nos leva até

$$
\begin{aligned}
& \frac{\delta}{\delta A_{\mu}} \mathcal{L}_{\text {eff }}^{(2)}=-\frac{e^{2} T^{2}}{2} \sum_{n_{p} n_{q}} \int \frac{d^{d-1} p}{(2 \pi)^{d-1}} \frac{d^{d-1} q}{(2 \pi)^{d-1}} \\
& \times \operatorname{tr}\left(\gamma^{\alpha} \frac{1}{\not_{p}} e \gamma^{\mu} \frac{1}{\not_{p}} \gamma_{\alpha} \frac{1}{\not_{q}}+\gamma^{\alpha} \frac{1}{\not_{p}} \gamma_{\alpha} \frac{1}{\not_{q}} e \gamma^{\mu} \frac{1}{\not_{q}}\right) \frac{1}{(p-q)^{2}}
\end{aligned}
$$

no segundo termos podemos fazer uma mudança de variáveis $(p, q) \rightarrow(q, p)$ e usar a ciclidade do traço para obter

$$
\frac{\delta}{\delta A_{\mu}} \mathcal{L}_{\text {eff }}^{(2)}=-e^{2} T^{2} \sum_{n_{p} n_{q}} \int \frac{d^{d-1} p}{(2 \pi)^{d-1}} \frac{d^{d-1} q}{(2 \pi)^{d-1}} \operatorname{tr}\left(\gamma^{\alpha} \frac{1}{\mathbb{t}_{p}} e \gamma^{\mu} \frac{1}{\mathbb{t}_{p}} \gamma_{\alpha} \frac{1}{\mathbb{t}_{q}}\right) \frac{1}{(p-q)^{2}},
$$

e calculando a segunda derivada obtemos

$$
\begin{aligned}
& \frac{\delta^{2}}{\delta A_{\nu} \delta A_{\mu}} \mathcal{L}_{\text {eff }}^{(2)}=-e^{2} T^{2} \sum_{n_{p} n_{q}} \int \frac{d^{d-1} p}{(2 \pi)^{d-1}} \frac{d^{d-1} q}{(2 \pi)^{d-1}}
\end{aligned}
$$

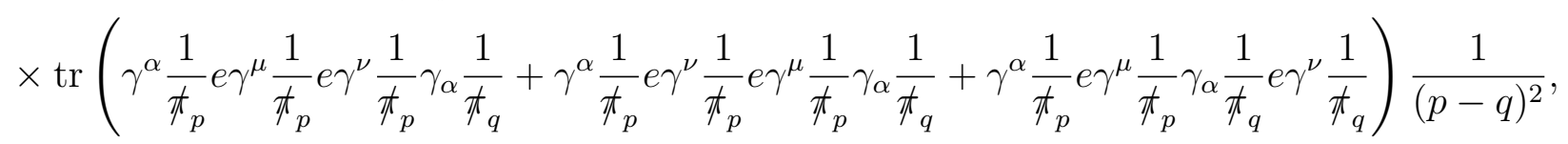

e assim teremos

$$
\begin{aligned}
\left.\frac{\delta^{2}}{\delta A_{\nu}^{C} \delta A_{\mu}^{C}} \mathcal{L}_{\text {eff }}^{(2)}\right|_{A^{C}=0} & =-\Pi_{2 a}^{\mu \nu}(k=0)-\Pi_{2 b}^{\mu \nu}(k=0) \\
& \simeq-\Pi_{2 a}^{\mu \nu}\left(k_{0}=0\right)-\Pi_{2 b}^{\mu \nu}\left(k_{0}=0\right) .
\end{aligned}
$$


190 Apêndice $F$ - Obtenção da auto-energia estática do fóton a partir da ação efetiva 


\section{APÊNDICE G - Alguns resultados básicos sobre campos em um espaço-tempo curvo}

Nesta seção seguiremos [PT09] e mostramos como construir as derivadas covariantes para um campo de spin arbitrário em um espaço-tempo curvo. Para tanto, usaremos também alguns resultados de [EGH80].

\section{G.1 Representações do grupo de Lorentz}

As transformações de Lorentz

$$
x^{a} \rightarrow \underline{x}^{a}=\Lambda_{b}^{a} x^{b}
$$

são construídas de modo a manter o produto escalar

$$
d S^{2}=\eta_{a b} d x^{a} d x^{b}=\eta_{c d} d \underline{x}^{a} d \underline{x}^{b}
$$

invariante, que implica que a métrica de Minkowski é invariante

$$
\eta_{a b} \Lambda_{c}^{a} \Lambda_{d}^{b}=\eta_{c d}
$$

$\mathrm{Ou}$

$$
\Lambda^{a c} \Lambda_{a d}=\delta_{b}^{a}
$$

que define a inversa de uma transformação de Lorentz é como sendo

$$
\left(\Lambda^{-1}\right)_{b}^{a}=\Lambda_{b}{ }^{a}
$$


Vetores contrariantes se transformam como um vetor posição

$$
X^{a} \rightarrow \underline{X}^{a}=\Lambda_{b}^{a} X^{b}
$$

que é a representação vetorial do grupo de Lorentz. O conjunto das transformações de Lorentz constituem um grupo de Lie. Logo, todos os elementos do grupo podem ser escritos como composições de transformações infinitesimais partindo continuamente da identidade. Escrevemos uma transformação de Lorentz infinitesimal como

$$
\Lambda_{b}^{a}=\delta_{b}^{a}+\delta \epsilon_{b}^{a},
$$

e a expressão da inversa (G.5) implica em

$$
\delta \epsilon_{a b}=-\delta \epsilon_{b a} .
$$

Uma representação do grupo de Lorentz atuando sobre objetos $\psi(x)$ é uma transformação

$$
\psi(x) \rightarrow \underline{\psi}(\underline{x})=D(\Lambda) \psi(x)
$$

que é compatível com a operação de grupo, no sentido que

$$
\begin{gathered}
D(\Lambda) D\left(\Lambda^{\prime}\right)=D\left(\Lambda \Lambda^{\prime}\right), \\
D\left(\Lambda^{-1}\right)=D(\Lambda)^{-1} .
\end{gathered}
$$

Para uma transformação infinitesimal teremos

$$
D(\Lambda)=\mathbb{1}+i \Sigma_{a b} \delta \epsilon^{a b}
$$

onde $\mathbb{1}$ é a matriz identidade nos índices internos de $\psi(x)$ e $\Sigma_{a b}=-\Sigma_{b a}$ é também uma matriz nos índices internos. As matrizes $\Sigma_{a b}$ são os geradores da transformação de Lorentz na representação $D(\Lambda)$.

Do fato que $D(\Lambda)$ é uma representação do grupo de Lorentz, segue que os geradores da representação $\Sigma_{a b}$ satisfazem a álgebra de Lorentz. Para ver isto, partimos de G.10a para escrever a identidade

$$
D(\Lambda) D\left(\Lambda^{\prime}\right) D\left(\Lambda^{-1}\right)=D\left(\Lambda \Lambda^{\prime} \Lambda^{-1}\right)
$$


Para $\Lambda_{a b}^{\prime}=\eta_{a b}+\delta \epsilon_{a b}^{\prime}$ infinitesimal, temos

$$
\left(\Lambda \Lambda^{\prime} \Lambda^{-1}\right)_{b}^{a}=\delta_{b}^{a}+\Lambda_{c}^{a} \delta \epsilon^{\prime c d} \Lambda_{b d}
$$

e usando G.11

$$
D\left(\Lambda \Lambda^{\prime} \Lambda^{-1}\right)=\mathbb{1}+i \Sigma_{a b} \Lambda_{c}^{a} \delta \epsilon^{\prime c d} \Lambda_{d}^{b}
$$

Por outro lado usando G.11 para $D\left(\Lambda^{\prime}\right)$ escrevemos

$$
D(\Lambda) D\left(\Lambda^{\prime}\right) D\left(\Lambda^{-1}\right)=\mathbb{1}+D(\Lambda) i \Sigma_{a b} D\left(\Lambda^{-1}\right) \delta \epsilon^{\prime a b}
$$

Portanto, substituindo G.14 e G.15 em G.12 e levando em conta a anti-simetria de $\delta \epsilon_{a b}^{\prime}$, obtemos

$$
D(\Lambda) \Sigma_{a b} D\left(\Lambda^{-1}\right)=\frac{1}{2}\left(\Lambda_{a}^{c} \Sigma_{c d} \Lambda_{b}^{d}-\Lambda_{b}^{c} \Sigma_{c d} \Lambda_{a}^{d}\right)
$$

Agora vamos considerar $\Lambda_{a b}=\eta_{a b}+\delta \epsilon_{a b}$ infinitesimal. Usando a expressão para a inversa

$$
\left(\Lambda^{-1}\right)_{a b}=\eta_{a b}-\delta \epsilon_{a b}
$$

até a ordem $\delta \epsilon$ teremos

$$
D(\Lambda) \Sigma_{a b} D\left(\Lambda^{-1}\right)=\Sigma_{a b}+i \delta \epsilon^{c d}\left[\Sigma_{c d}, \Sigma_{a b}\right]
$$

e

$$
\begin{aligned}
\Lambda_{a}^{c} \Sigma_{c d} \Lambda_{b}^{d} & =\left(\delta_{a}^{c}+\delta \epsilon_{a}^{c}\right) \Sigma_{c d}\left(\delta_{b}^{d}+\delta \epsilon_{b}^{d}\right) \\
& =\Sigma_{a b}+\frac{1}{2}\left(\eta_{a d} \Sigma_{c b}-\eta_{a c} \Sigma_{d b}-\eta_{b c} \Sigma_{a d}+\eta_{b d} \Sigma_{a c}\right) \delta \epsilon^{c d} .
\end{aligned}
$$

Substituindo G.18 e G.19 em G.16 obtemos

$$
\left[\Sigma_{a b}, \Sigma_{c d}\right]=\frac{i}{2}\left(\eta_{a d} \Sigma_{c b}-\eta_{a c} \Sigma_{d b}-\eta_{b c} \Sigma_{a d}+\eta_{b d} \Sigma_{a c}\right)
$$

que é a álgebra de Lie dos geradores das transformações de Lorentz ou simplesmente álgebra de Lorentz.

A representação mais direta do grupo de Lorentz é a representação vetorial G.6. 
Para esta representação, escrevendo uma transformação infinitesimal

$$
\begin{aligned}
\Lambda_{a b} & =\eta_{a b}+\delta \epsilon_{a b} \\
& =\eta_{a b}+\frac{1}{2}\left[\eta_{a c} \eta_{b d}-\eta_{a d} \eta_{b c}\right] \delta \epsilon^{c d}
\end{aligned}
$$

e comparando com G.11, vemos que os geradores desta representação possuem a forma

$$
\left(\Sigma_{a b}\right)_{c d}=\frac{1}{2 i}\left[\eta_{a c} \eta_{b d}-\eta_{a d} \eta_{b c}\right]
$$

e, neste caso, os índices internos são índices de Lorentz. Como um exercício simples podemos verificar que os geradores G.22 satisfazem a álgebra G.20. De fato, computando o produto matricial obtemos

$$
\begin{aligned}
{\left[\Sigma_{a b}, \Sigma_{c d}\right]_{e g} } & =\left(\Sigma_{a b}\right)_{e}^{f}\left(\Sigma_{c d}\right)_{f g}-\left(\Sigma_{c d}\right)_{e}^{f}\left(\Sigma_{a b}\right)_{f g} \\
& =\frac{i}{2}\left[\eta_{a d}\left(\Sigma_{c b}\right)_{e g}-\eta_{a c}\left(\Sigma_{d b}\right)_{e g}-\eta_{b c}\left(\Sigma_{a d}\right)_{e g}+\eta_{b d}\left(\Sigma_{a c}\right)_{e g}\right]
\end{aligned}
$$

que é a álgebra de Lorentz.

\section{G.1.1 Campo de Dirac}

Para escrever os geradores das transformações de Lorentz na representação espinorial, vamos usar a álgebra das matrizes de Dirac

$$
\left\{\gamma^{a}, \gamma^{b}\right\}=2 \eta^{a b}
$$

e as identidades dos comutadores

$$
\begin{gathered}
{[a b, c]=a[b, c]+[a, c] b} \\
{[a, b c]=\{a, b\} c-b\{a, c\}}
\end{gathered}
$$

para obter

$$
\begin{aligned}
{\left[\gamma^{a} \gamma^{b}, \gamma^{c} \gamma^{d}\right] } & =\gamma^{a}\left[\gamma^{b}, \gamma^{c} \gamma^{d}\right]+\left[\gamma^{a}, \gamma^{c} \gamma^{d}\right] \gamma^{b} \\
& =\gamma^{a}\left\{\gamma^{b}, \gamma^{c}\right\} \gamma^{d}-\gamma^{a} \gamma^{c}\left\{\gamma^{b}, \gamma^{d}\right\}+\left\{\gamma^{a}, \gamma^{c}\right\} \gamma^{d} \gamma^{b}-\gamma^{c}\left\{\gamma^{a}, \gamma^{d}\right\} \gamma^{b} \\
& =2\left(\eta^{b c} \gamma^{a} \gamma^{d}-\eta^{b d} \gamma^{a} \gamma^{c}+\eta^{a c} \gamma^{d} \gamma^{b}-\eta^{a d} \gamma^{c} \gamma^{b}\right)
\end{aligned}
$$

O uso sucessivo de (G.26) fornece

$$
\left[\left[\gamma^{a}, \gamma^{b}\right],\left[\gamma^{c}, \gamma^{d}\right]\right]=4\left(\eta^{b c}\left[\gamma^{a}, \gamma^{d}\right]-\eta^{b d}\left[\gamma^{a}, \gamma^{c}\right]+\eta^{a c}\left[\gamma^{d}, \gamma^{b}\right]-\eta^{a d}\left[\gamma^{c}, \gamma^{b}\right]\right)
$$


Logo, somos motivados a definir

$$
\sigma^{a b}=-\frac{i}{8}\left[\gamma^{a}, \gamma^{b}\right]
$$

de tal maneira que

$$
\sigma^{a b}=-\sigma^{b a}
$$

e

$$
\sigma^{a b}=-\frac{i}{4} \gamma^{a} \gamma^{b}
$$

sempre que $a \neq b$. Além disso, multiplicando G.27) por $(-i / 8)^{2}$ obtemos que $\sigma^{a b}$ satisfaz

$$
\left[\sigma^{a b}, \sigma^{c d}\right]=\frac{i}{2}\left(\eta^{a d} \sigma^{c b}-\eta^{a c} \sigma^{d b}-\eta^{b c} \sigma^{a d}+\eta^{b d} \sigma^{a c}\right)
$$

que é a álgebra dos geradores das transformações de Lorentz G.20 . Portanto, $\sigma^{a b}$ definidos em (G.28), são os geradores da representação espinorial do grupo de Lorentz. Logo, seguindo (G.11), vemos que uma transformação de Lorentz infinitesimal possui a forma

$$
D(\delta \Lambda)=\mathbb{1}+i \sigma^{a b} \delta \epsilon_{a b},
$$

e uma transformação finita será então um sucessão de transformações infinitesimais na forma

$$
D(\Lambda)=\lim _{N \rightarrow \infty}\left(\mathbb{1}+\frac{i \sigma^{a b} \epsilon_{a b}}{N}\right)^{N}=\exp \left(i \sigma^{a b} \epsilon_{a b}\right),
$$

e, consequentemente, o espinor se transforma por transformação de Lorentz como

$$
\psi(x) \rightarrow D(\Lambda) \psi(\Lambda x)
$$

Por consistência, a equação de Dirac deve ser covariante por transformações de Lorentz. A equação de Dirac

$$
\left(i \gamma^{a} \partial_{a}-m\right) \psi(x)=0
$$

é escrita em um novo referencial como

$$
\left[i \gamma^{a} \Lambda_{a}^{b} \partial_{b}-m\right] D(\Lambda) \psi(\Lambda x)=0
$$


Colocando em evidencia o operador $D(\Lambda)$ teremos

$$
D(\Lambda)\left[i D^{-1}(\Lambda) \gamma^{a} D(\Lambda) \Lambda_{a}^{b} \partial_{b}-m\right] \psi(\Lambda x)=0
$$

Para uma transformação infinitesimal, teremos

$$
\begin{aligned}
D^{-1}(\delta \Lambda) \gamma^{a} D(\delta \Lambda) & =\left(\mathbb{1}-i \delta \epsilon_{c d} \sigma^{c d}\right) \gamma^{a}\left(\mathbb{1}-i \delta \epsilon_{e f} \sigma^{e f}\right) \\
& =\gamma^{a}+i \delta \epsilon_{c d}\left[\gamma^{a}, \sigma^{c d}\right]
\end{aligned}
$$

Avaliando o comutador

$$
\begin{aligned}
{\left[\gamma^{a}, \sigma^{c d}\right] } & =\frac{i}{8}\left(\left[\gamma^{a}, \gamma^{c} \gamma^{d}\right]-[(c, d) \rightarrow(d, c)]\right) \\
& =\frac{i}{8}\left(\left\{\gamma^{a}, \gamma^{c}\right\} \gamma^{d}-\gamma^{c}\left\{\gamma^{a}, \gamma^{d}\right\}-[(c, d) \rightarrow(d, c)]\right) \\
& =\frac{i}{2}\left(\eta^{a d} \gamma^{c}-\eta^{a c} \gamma^{d}\right)
\end{aligned}
$$

obtemos

$$
D^{-1}(\delta \Lambda) \gamma^{a} D(\delta \Lambda)=\gamma^{a}-\frac{1}{2} \delta \epsilon_{c d}\left(\eta^{a d} \gamma^{c}-\eta^{a c} \gamma^{d}\right)=\left(\delta_{b}^{a}+\delta \epsilon_{a b}\right) \gamma^{b}=\delta \Lambda_{b}^{a} \gamma^{b} .
$$

Repetindo o processo indefinidamente, obtemos para uma transformação finita

$$
D^{-1}(\Lambda) \gamma^{a} D(\Lambda)=\Lambda_{b}^{a} \gamma^{b}
$$

Neste sentido acima, $\gamma^{a}$ se comporta como vetor por transformações de Lorentz. Substituindo na equação de Dirac no novo referencial (G.37), obtemos

$$
D(\Lambda)\left[i \gamma^{c} \Lambda^{a}{ }_{c} \Lambda_{a}{ }^{b} \partial_{b}-m\right] \psi(\Lambda x)=0,
$$

Usando a inversa da transformação de Lorentz G.5 teremos

$$
D(\Lambda)\left[i \gamma^{a} \partial_{a}-m\right] \psi(\Lambda x)=0
$$

que expressa a covariância da equação de Dirac.

De maneira semelhante, podemos mostrar que a lagrangiana de Dirac

$$
\mathcal{L}_{d}=\bar{\psi}(x)\left(i \gamma^{a} \partial_{a}-m\right) \psi(x)
$$


é um escalar. Para isto, note que $\bar{\psi}(x)=\psi^{\dagger}(x) \gamma^{0}$ se transforma por Lorentz.

$$
\begin{aligned}
\bar{\psi}(x) & \rightarrow \bar{\psi}^{\prime}(\Lambda x) \\
& =\psi^{\dagger}(\Lambda x) D^{\dagger}(\Lambda) \gamma^{0}
\end{aligned}
$$

Os geradores da da representação espinorial do grupo de Lorentz G.28 não são hermitianos. Logo, a representação espinorial é não unitária. Deste modo teremos

$$
D^{\dagger}(\Lambda)=e^{-i\left(\Sigma^{a b}\right)^{\dagger} \Lambda_{a b}}
$$

Os geradores dos "boosts" são anti-hermitianos, ou seja

$$
\begin{aligned}
\left(\Sigma^{0 i}\right)^{\dagger} & =-\frac{i}{4}\left(\gamma^{i}\right)^{\dagger}\left(\gamma^{0}\right)^{\dagger} \\
& =\frac{i}{4} \gamma^{i} \gamma^{0} \\
& =-\Sigma^{0 i}
\end{aligned}
$$

e portanto teremos

$$
\left(\Sigma^{0 i}\right)^{\dagger} \gamma^{0}=\gamma^{0} \Sigma^{0 i}
$$

Já os geradores das rotações $\Sigma^{i j}$, com $i \neq j$, são hermitianos

$$
\begin{aligned}
\left(\Sigma^{i j}\right)^{\dagger} & =-\frac{i}{4}\left(\gamma^{j}\right)^{\dagger}\left(\gamma^{i}\right)^{\dagger} \\
& =-\frac{i}{4} \gamma^{j} \gamma^{i} \\
& =\Sigma^{i j}
\end{aligned}
$$

de modo que

$$
\left(\Sigma^{i j}\right)^{\dagger} \gamma^{0}=\gamma^{0} \Sigma^{i j}
$$

Portanto, substituindo as equações G.46, G.48 e G.50 em G.45, vemos que $\bar{\psi}$ se transforma por Lorentz como

$$
\bar{\psi}(x) \rightarrow \bar{\psi}(\Lambda x)[D(\Lambda)]^{-1}
$$

o que implica na invariância de Lorentz da lagrangiana de Dirac. 


\section{G.2 O formalismo das tetradas}

Considere um variedade pseudo-riemanniana d-dimensional $\mathcal{M}$, dotada de um tensor métrico de signatura $+,-, \cdots,-$. Suponha que este espaço seja livre de torção, implicando que a conexão seja simétrica e dada pela conexão métrica ou símbolo de Christoffel. O elemento de linha em um sistema de coordenadas $x^{\mu}$ possui a forma

$$
d S^{2}=g_{\mu \nu}(x) d x^{\mu} d x^{\nu}
$$

Considere um transformação de coordenadas

$$
x^{\mu} \rightarrow y^{a}(x)
$$

Definimos as matrizes de transformação de coordenadas, ou tetradas, como

$$
e_{\mu}^{a}(x)=\left.\frac{\partial y^{a}}{\partial x^{\mu}}\right|_{x},
$$

$\mathrm{e}$

$$
E_{a}{ }^{\mu}(x)=\left.\frac{\partial x^{\mu}}{\partial y^{a}}\right|_{x},
$$

onde estamos abusando da notação e dizendo que $x$ é o ponto $P$ da variedade $M$ cujas coordenadas são $x^{\mu}(P)$. No sistema $y^{a}$ a métrica possui a forma

$$
g_{a b}^{\prime}(y)=E_{a}^{\mu}(x) E_{b}^{\nu}(x) g_{\mu \nu}(x)
$$

e a transformação inversa possui a forma

$$
g_{\mu \nu}(x)=e^{a}{ }_{\mu}(x) e^{b}{ }_{\nu}(x) g_{a b}^{\prime}(y)
$$

A ideia do formalismo das tetradas consiste em impor que a tetrada $e^{a}{ }_{\mu}(x)$ seja localmente uma "raiz quadrada" da métrica, no sentido que

$$
g_{\mu \nu}(x)=e^{a}{ }_{\mu}(x) e^{b}{ }_{\nu}(x) \eta_{a b}
$$

assim, tiramos proveito do princípio da equivalência para encontrar um sistema de coordenadas em que a métrica é localmente igual a métrica de Minkowski. Na presença de um campo gravitacional não uniforme, teremos que não existe um único sistema de coordenadas em que a métrica é a métrica de Minkowski em cada ponto da variedade, caso contrario o espaço-tempo seria plano. Assim, temos que a construção das tetradas 
é local e a rigor, teremos un! sistema de coordenadas localmente plano para cada ponto do espaço tempo.

Em nossa notação os índices $a, b, \cdots$ estarão relacionados com o sistema de coordenadas localmente plano serão manipulados com a métrica plana $\eta_{a b}$, enquanto os índices $\mu, \nu, \cdots$ são índices de tranformação geral de coordenadas e serão abaixados e levantados usando a métrica $g_{\mu \nu}$. Deste modo teremos

$$
E_{a}^{\mu}(x)=\eta_{a b} g^{\mu \nu}(x) e^{b}{ }_{\nu}(x)
$$

e

$$
E_{a}{ }^{\mu}(x) e^{a}{ }_{\nu}(x)=\delta_{\nu}^{\mu}, \quad E_{a}{ }^{\mu}(x) e^{b}{ }_{\mu}(x)=\delta_{a}^{b} .
$$

Em termos das tetradas, o elemento de linha possui a forma

$$
d S^{2}=\eta_{a b} e^{a}{ }_{\mu}(x) e^{b}{ }_{\nu}(x) d x^{\mu} d x^{\nu},
$$

e definindo

$$
e^{a}{ }_{\mu}(x) d x^{\mu}=e^{a}(x)
$$

reescrevemos

$$
d S^{2}=\eta_{a b} e^{a}(x) e^{b}(x)
$$

Sabemos que o conjunto $\left\{d x^{\mu}\right\}$ forma uma base para o espaço cotangente $T_{x}^{*}(M)$ a variedade $M$ no ponto $x$. Logo, vemos que a tetrada $e^{a}{ }_{\mu}(x)$ transforma a base coordenada $d x^{\mu}$ em uma base ortonormal $e^{a}(x)$. Entretanto, usando a linguagem das formas diferenciais de Cartan, vemos que enquanto os diferenciais $d x^{\mu}$ são diferenciais exatos, temos que

$$
d e^{a}(x)=\frac{1}{2}\left[\partial_{\mu} e^{a}{ }_{\mu}(x)-\partial_{\nu} e^{a}{ }_{\mu}\right] d x^{\mu} \wedge d x^{\nu},
$$

são não nulos em geral, implicando que $e^{a}$ não são diferenciais exatos. Usamos a notação em que

$$
d x^{\mu} \wedge d x^{\nu}=\frac{1}{2}\left[d x^{\mu} \otimes d x^{\nu}-d x^{\nu} \otimes d x^{\mu}\right]
$$

é o produto exterior de Cartan.

De maneira equivalente temos que $E_{a}{ }^{\mu}(x)$ transforma a base coordenada $\partial_{\mu}$ do espaço

\footnotetext{
${ }^{1} \mathrm{~A}$ menos de uma transformação de Lorentz local.
} 
tangente $T_{x}(M)$ em uma base ortonormal $E_{a}(x)$ por

$$
E_{a}(x)=E_{a}{ }^{\mu}(x) \partial_{\mu}
$$

e enquanto as derivadas parciais $\partial_{\mu}$ comutam, temos que o parêntese de Lie

$$
\left[E_{a}(x), E_{b}(x)\right]=\left\{E_{a}^{\mu}(x)\left[\partial_{\mu} E_{b}^{\nu}(x)\right]-E_{b}^{\mu}(x)\left[\partial_{\mu} E_{b}^{\nu}(x)\right]\right\} \partial_{\nu}
$$

é não nulo o que significa que esta base é não holonômica.

Mesmo que fixado o sistema de coordenadas de partida $x^{\mu}$, as tetradas não são unicamente definidas por G.58. Como as transformações de Lorentz matem a métrica de Minkowski invariante, fazendo

$$
\underline{e}_{\mu}^{a}(x)=\Lambda_{b}^{a}(x) e_{\mu}^{b}(x)
$$

vemos que

$$
g_{\mu \nu}(x)=\underline{e}_{\mu}^{a}(x) \underline{e}_{\nu}^{b}(x) \eta_{a b}=e_{\mu}^{a}(x) e_{\nu}^{b}(x) \eta_{a b},
$$

de modo que as tetradas são definidas a menos de uma transformação de Lorentz local $\Lambda_{b}^{a}(x)$, em que os parâmetros da transformação de Lorentz variam no espaço-tempo.

Se fizermos uma transformação geral de coordenadas

$$
x^{\mu} \rightarrow x^{\prime \mu}
$$

não temos nenhuma razão para supor que o sistema de coordenadas local fique inalteradq2 Deste modo, sobre transformação geral de coordenadas as tetradas se transformam como

$$
e^{\prime a}{ }_{\mu}\left(x^{\prime}\right)=\Lambda_{b}^{a}(x) \frac{\partial x^{\nu}}{\partial x^{\prime \mu}} e^{b}{ }_{\nu}(x)
$$

Um vetor contravariante possui uma lei de transformação dada por

$$
V^{\prime \mu}\left(x^{\prime}\right)=\frac{\partial x^{\prime \mu}}{\partial x^{\nu}} V^{\mu}(x)
$$

Escrevemos este vetor na base da tetrada

$$
V^{a}(x)=e^{a}{ }_{\mu}(x) V^{\mu}(x)
$$

\footnotetext{
${ }^{2}$ Seguimos as definições das tetradas tais como feitas em [PT09]. Outros autores [Wei72, BD94] preferem manter o sistema de coordenadas localmente inercial invariante por transformações geral de coordenadas, resultando em uma definição diferente da derivada covariante das tetradas G.83. Entretanto, ambos métodos são equivalentes.
} 
e a equação G.71 implica que teremos a seguinte lei de transformação

$$
V^{\prime a}\left(x^{\prime}\right)=\Lambda_{b}^{a}(x) V^{b}(x)
$$

A equação G.55 que define a inversa da tetrada juntamente com a equação G.71) para a transformação da tetrada, implicam na seguinte lei de transformação para a inversa da tetrada

$$
E^{\prime}{ }_{a}^{\mu}\left(x^{\prime}\right)=\Lambda_{a}^{b}(x) \frac{\partial x^{\prime \mu}}{\partial x^{\nu}} E_{b}^{\nu}(x)
$$

Um tensor geral pode ser escrito na base das tetradas de maneira natural

$$
T_{b_{1} \cdots b_{m}}^{a_{1} \cdots a_{n}}(x)=e^{a_{1}}{ }_{\mu_{1}}(x) \cdots e^{a_{n}}{ }_{\mu_{n}}(x) E_{b_{1}}{ }^{\nu_{1}}(x) \cdots E_{b_{m}}{ }^{\nu_{m}}(x) T_{\nu_{1} \cdots \nu_{m}}^{\mu_{1} \cdots \mu_{n}}(x),
$$

de modo que usando as equações G.71 e G.75 obtemos a seguinte lei de transformação

$$
T_{b_{1} \cdots b_{m}}^{\prime a_{1} \cdots a_{n}}\left(x^{\prime}\right)=\Lambda_{c_{1}}^{a_{1}}(x) \cdots \Lambda_{c_{1}}^{a_{1}}(x) \Lambda_{b_{1}}{ }^{d_{1}}(x) \cdots \Lambda_{b_{m}}{ }^{d_{m}}(x) T_{d_{1} \cdots d_{m}}^{c_{1} \cdots c_{n}}(x)
$$

A derivada covariante do um vetor na base da tetrada deve ter a seguinte lei de transformação

$$
\nabla_{a}^{\prime} V^{\prime b}\left(x^{\prime}\right)=\Lambda_{a}^{c}(x) \Lambda_{d}^{b}(x) \nabla_{c} V^{d}(x)
$$

ou escrevendo $\nabla_{a}=e_{a}{ }^{\nu} \nabla_{\nu}$ temos equivalentemente

$$
\nabla_{\nu}^{\prime} V^{\prime b}\left(x^{\prime}\right)=\frac{\partial x^{\mu}}{\partial x^{\prime \nu}} \Lambda_{d}^{b}(x) \nabla_{\mu} V^{d}(x)
$$

Portanto definindo a derivada covariante como

$$
\nabla_{a} V^{b}(x)=E_{a}{ }^{\mu}(x) \nabla_{\mu} V^{b}(x)=E_{a}{ }^{\mu}(x)\left(\partial_{\mu} V^{b}(x)+\omega_{\mu}{ }_{c}^{b}(x) V^{c}(x)\right),
$$

obtemos que as conexões de spin $\omega_{\mu}{ }_{c}^{b}(x)$ obedecem a seguinte lei de transformação

$$
\begin{aligned}
\omega_{\mu b}^{\prime a}\left(x^{\prime}\right) & =\frac{\partial x^{\nu}}{\partial x^{\prime \mu}}\left[\Lambda_{c}^{a}(x) \Lambda_{b}{ }^{d}(x) \omega_{\nu d}^{c}(x)-\Lambda_{b}{ }^{c}(x) \partial_{\nu} \Lambda_{c}^{a}{ }_{c}(x)\right] \\
& =\frac{\partial x^{\nu}}{\partial x^{\prime \mu}}\left[\Lambda^{a}{ }_{c}(x) \Lambda_{b}{ }^{d}(x) \omega_{\nu}{ }^{c}{ }_{d}(x)+\Lambda^{a}{ }_{c}(x) \partial_{\nu} \Lambda_{b}{ }^{c}(x)\right]
\end{aligned}
$$

Usando a definição G.80 e impondo a regra de Leibeniz para a derivada covariante

$$
\nabla_{\mu} V^{a}(x)=\nabla_{\mu}\left[e^{a}{ }_{\nu}(x) V^{\nu}(x)\right]=\nabla_{\mu}\left[e^{a}{ }_{\nu}(x)\right] V^{\nu}(x)+e^{a}{ }_{\nu}(x) \nabla_{\mu}\left[V^{\nu}(x)\right]
$$


obtemos que a derivada covariante da tetrada possui a forma

$$
\nabla_{\mu} e_{\nu}^{a}(x)=\partial_{\mu} e_{\nu}^{a}(x)+\omega_{\mu}^{a}{ }_{b} e_{\nu}^{b}(x)-\Gamma_{\mu \nu}^{\lambda} e_{\lambda}^{a}(x)
$$

onde usamos

$$
\nabla_{\mu} V^{\nu}=\partial_{\mu} V^{\nu}+\Gamma_{\mu \alpha}^{\nu} V^{\alpha}
$$

e $\Gamma_{\mu \alpha}^{\nu}$ é a conexão métrica, ou símbolo de Christoffel. Note que a definição G.83) é covariante tanto em relação a transformações gerais de coordenadas quanto a transformações de Lorentz no sistema localmente inercial.

Embora conexão não possua um carácter tensorial, sua parte anti-simétrica

$$
T_{\mu \nu}^{\lambda}=\frac{1}{2}\left(\Gamma_{\mu \nu}^{\lambda}-\Gamma_{\nu \mu}^{\lambda}\right)
$$

é um tensor chamado de torção. Neste trabalho estaremos sempre no contexto de variedades descritas pela teoria da relatividade geral de Einstein, de modo trabalhamos em espaços em que a torção é nula. Para variedades livres de torção, temos que as geodésicas afim e métrica coincidem, e a conexão afim é idêntica a conexão métrica ou símbolos de Christoffel, o que implica que a derivada covariante da métrica é nula d'I92.

Segundo a definição G.58), a tetrada é uma especie de "raiz quadrada" da métrica e, portanto, é natural que para espaços livres de torção tenhamos

$$
\nabla_{\mu} e^{a}{ }_{\nu}(x)=0
$$

ou seja, a derivada convariante de terada é nula (para uma prova deste fato veja [EGH80]). A equação G.83 juntamente com G.86 fixam a forma da conexão de spin como sendo

$$
\omega_{\mu b}^{a}=\Gamma_{\mu \nu}^{\lambda} e_{\lambda}^{a} E_{b}^{\nu}-E_{b}^{\nu} \partial_{\mu} e_{\nu}^{a}
$$

Esta conexão de spin é anti-simétrica nos índices $a$ e $b$. Para ver isto vamos abaixar o índice $a$ com a métrica me Minkowski

$$
\omega_{\mu a b}=\Gamma_{\mu \nu}^{\lambda} e_{a \lambda} E_{b}{ }^{\nu}-E_{b}{ }^{\nu} \partial_{\mu} e_{a \nu}
$$

e usar as identidades

$$
\begin{aligned}
E_{b}{ }^{\nu} \partial_{\mu} e_{a \nu} & =E_{b}{ }^{\nu} \partial_{\mu}\left[E_{a}{ }^{\lambda} g_{\nu \lambda}\right] \\
& =E_{b}{ }^{\nu} E_{a}{ }^{\lambda} \partial_{\mu} g_{\nu \lambda}+e_{b \nu} \partial_{\mu} E_{a}{ }^{\nu}
\end{aligned}
$$


e

$$
E_{b}{ }^{\nu} \partial_{\mu} e_{a \nu}=-e_{a \nu} \partial_{\mu} E_{b}{ }^{\nu}
$$

de modo que somando G.89 a G.90

$$
E_{b}{ }^{\nu} \partial_{\mu} e_{a \nu}=\frac{1}{2}\left[E_{b}{ }^{\nu} E_{a}{ }^{\lambda} \partial_{\mu} g_{\nu \lambda}+e_{b \nu} \partial_{\mu} E_{a}{ }^{\nu}-e_{a \nu} \partial_{\mu} E_{b}{ }^{\nu}\right]
$$

Substituindo G.91 em G.88 e usando a expressão da conexão métrica d'I92, obemos

$$
\begin{aligned}
\omega_{\mu a b} & =\frac{1}{2}\left[\partial_{\mu} g_{\lambda \nu}+\partial_{\nu} g_{\lambda \mu}-\partial_{\lambda} g_{\mu \nu}\right] E_{a}{ }^{\lambda} E_{b}{ }^{\nu}-\frac{1}{2}\left[E_{b}{ }^{\nu} E_{a}{ }^{\lambda} \partial_{\mu} g_{\nu \lambda}+e_{b \nu} \partial_{\mu} E_{a}{ }^{\nu}-e_{a \nu} \partial_{\mu} E_{b}{ }^{\nu}\right] \\
& =\frac{1}{2}\left[\partial_{\nu} g_{\lambda \mu}-\partial_{\lambda} g_{\mu \nu}\right] E_{a}{ }^{\lambda} E_{b}{ }^{\nu}-\frac{1}{2}\left[e_{b \nu} \partial_{\mu} E_{a}{ }^{\nu}-e_{a \nu} \partial_{\mu} E_{b}{ }^{\nu}\right],
\end{aligned}
$$

que é explicitamente anti-simétrico nos índices $a$ e $b$, de modo que

$$
\omega_{\mu a b}=-\omega_{\mu b a} .
$$

\section{G.3 Derivada covariante para representações do grupo de Lorentz}

Suponha que um objeto $\xi(x)$ esteja escrito na base das tetradas, no sentido que ele seja um escalar por transformações gerais de coordenadas puras que mantém a base da tetrada invariante. Suponha também que por transformações de Lorentz locais puras $\xi(x)$ se transforme por uma representação do grupo de Lorentz $D[\Lambda(x)]$ :

$$
\xi^{\prime}(x)=D[\Lambda(x)] \xi(x)
$$

cujos geradores são $\Sigma^{a b}$. Para uma transformação infinitesimal

$$
\delta \Lambda^{a}{ }_{b}(x)=\delta_{b}^{a}+\delta \epsilon^{a}{ }_{b}(x),
$$

teremos

$$
D[\delta \Lambda(x)]=\mathbb{1}+i \Sigma^{a b} \delta \epsilon_{a b}(x)
$$

onde os geradores da transformação obedecem a álgebra de Lie do grupo de Lorentz G.20). Em um grupo de Lie todos os elementos podem ser escritos como composição de 
transformações infinitesimais. Assim, para um parâmetro finito $\epsilon_{a b}=N \delta \epsilon_{a b}$ teremos

$$
D[\Lambda(x)]=\lim _{N \rightarrow \infty}\left[\mathbb{1}+i \frac{1}{N} \Sigma^{a b} \epsilon_{a b}(x)\right]^{N}=\exp \left[i \Sigma^{a b} \epsilon_{a b}(x)\right] .
$$

Vamos construir a derivada covariante

$$
\nabla_{\mu} \xi(x)=\partial_{\mu} \xi(x)+B_{\mu}(x) \xi(x)
$$

impondo que ela seja, de fato, covariante por transformações de Lorentz locais, isto é

$$
\nabla_{\mu}^{\prime} \xi^{\prime}(x)=D[\Lambda(x)] \nabla_{\mu} \xi(x)
$$

Do lado esquerdo teremos

$$
\begin{aligned}
\nabla_{\mu}^{\prime} \xi^{\prime}(x) & =\left[\partial_{\mu}+B_{\mu}^{\prime}(x)\right] D[\Lambda(x)] \xi(x) \\
& =D[\Lambda(x)] \partial_{\mu} \xi(x)+\left\{\partial_{\mu} D[\Lambda(x)]\right\} \xi(x)+B_{\mu}^{\prime}(x) D[\Lambda(x)] \xi(x),
\end{aligned}
$$

enquanto do lado direito teremos

$$
D[\Lambda(x)] \nabla_{\mu} \xi(x)=D[\Lambda(x)] \partial_{\mu} \xi(x)+D[\Lambda(x)] B_{\mu}(x) \xi(x)
$$

e igualando estas expressões vemos que as conexões se transformam como

$$
B_{\mu}^{\prime}(x)=D[\Lambda(x)] B_{\mu}(x) D^{-1}[\Lambda(x)]-\left\{\partial_{\mu} D[\Lambda(x)]\right\} D^{-1}[\Lambda(x)]
$$

Deste modo, para uma transformação infinitesimal G.96) teremos

$$
B_{\mu}^{\prime}(x)=B_{\mu}(x)+i \delta \epsilon_{a b}(x)\left[\Sigma^{a b}, B_{\mu}(x)\right]-i \Sigma^{a b} \partial_{\mu} \delta \epsilon_{a b}(x) .
$$

Como estas conexões $B_{\mu}(x)$ são relativas a derivada covariante da representação $D[\Lambda(x)]$, elas devem ser proporcionais aos geradores desta representação do grupo de Lorentz ${ }^{3}$, isto é

$$
B_{\mu}(x)=B_{\mu a b}(x) \Sigma^{a b}
$$

com $B_{\mu a b}=-B_{\mu b a}$. Substituindo em G.103 usando a álgebra de Lie G.20 obtemos

$$
B_{\mu a b}^{\prime}=B_{\mu a b}+B_{\mu b c} \delta \epsilon_{a}^{c}-B_{\mu a c} \delta \epsilon_{b}^{c}-i \partial_{\mu} \delta \epsilon_{a b}
$$

A equação G.81 implica que as conexões de spin $\omega_{\mu}^{a}{ }_{b}(x)$ transformam-se por uma trans-

\footnotetext{
${ }^{3}$ Não temos elementos para construir a conexão senão os geradores.
} 
formação de Lorentz local como

$$
\omega_{\mu b}^{\prime a}(x)=\Lambda_{c}^{a}(x) \Lambda_{b}{ }^{d}(x) \omega_{\mu}^{c}{ }_{d}^{c}(x)+\Lambda_{c}^{a}(x) \partial_{\mu} \Lambda_{b}^{c}(x) .
$$

Fazendo $\Lambda(x)$ infinitesimal obtemos

$$
\omega_{\mu b}^{\prime a}=\left(\delta_{c}^{a}+\delta \epsilon_{c}^{a}\right)\left(\delta_{b}^{d}+\delta \epsilon_{b}{ }^{d}\right) \omega_{\mu_{d}{ }^{c}}+\left(\delta_{c}^{a}+\delta \epsilon^{a}{ }_{c}\right) \partial_{\mu}\left(\delta_{b}^{c}+\delta \epsilon_{b}{ }^{c}\right),
$$

e abaixando o índice $a$ com a métrica de Minkowski, teremos até ordem $\epsilon$

$$
\omega_{\mu a b}^{\prime}=\omega_{\mu a b}+\omega_{\mu b c} \delta \epsilon_{a}^{c}-\omega_{\mu a c} \delta \epsilon_{b}^{c}-\partial_{\mu} \delta \epsilon_{a b} .
$$

Logo, comparando as equações G.108 e G.105, obtemos

$$
B_{\mu a b}=i \omega_{\mu a b}
$$

e a derivada covariante assume a forma

$$
\nabla_{\mu} \psi=\left(\partial_{\mu}+i \omega_{\mu a b} \Sigma^{a b}\right) \psi
$$

e na base das tetradas escrevemos

$$
\nabla_{c} \psi=E_{c}{ }^{\mu} \nabla_{\mu} \psi=E_{c}{ }^{\mu}\left(\partial_{\mu}+i \omega_{\mu a b} \Sigma^{a b}\right) \psi=\left(\partial_{c}+i \omega_{c a b} \Sigma^{a b}\right) \psi
$$




\section{APÊNDICE H - Funções de Green do campo gravitacional}

Neste apêndice obteremos os termos de primeira e segunda ordem na equação (7.10), que são as funções de Green de um e dois pontos do campo gravitacional [BS12a].

Para o termo de primeira ordem, vamos notar que o traço de $I_{\mu \nu}$ na equação (7.11),

$$
\eta_{\mu \nu} I^{\mu \nu}=-\frac{T}{2} \sum_{n=-\infty}^{\infty} \int \frac{d^{d-1} p}{(2 \pi)^{d-1}},
$$

é igual a zero no cotexto de regularização zeta, usando 7.24. Logo, denotando $u^{\mu}$ pelo quadri-vetor que caracteriza o banho térmico, a forma mais geral para $I_{\mu \nu}$ é

$$
I_{\mu \nu}=a\left(d u_{\mu} u_{\nu}-\eta_{\mu \nu}\right)
$$

Como $u_{\mu}$ é uma quadri-velocidade temos que $\eta^{\mu \nu} u_{\mu} u_{\nu}=1$, e no referencial de repouso do banho térmico temos $u_{\mu}=(1,0,0, \cdots, 0)$. Projetando os dois lados da equação tensor $u^{\mu} u^{\nu}$ e usando a equação (7.11) obtemos

$$
a=-\frac{T}{2} \frac{1}{d-1} \sum_{n=-\infty}^{\infty} \int \frac{d^{d-1} p}{(2 \pi)^{d-1}} \frac{p_{0}^{2}}{p^{2}} .
$$

Usando a identidade algébrica $p_{0}^{2}=p^{2}+\vec{p}^{2}$ e desprezando os termos independentes da temperatura obtemos

$$
a=\frac{1}{2} \frac{1}{d-1} \int \frac{d \Omega_{d-1}}{(2 \pi)^{d-1}} \int_{0}^{\infty}|\vec{p}|^{d} S^{1}(|\vec{p}|) d|\vec{p}|,
$$

onde

$$
\begin{aligned}
S^{1}(|\vec{p}|) & =T \sum_{n=-\infty}^{\infty} \frac{1}{(2 \pi n T)^{2}+\vec{p}^{2}} \\
& =\frac{1}{2|\vec{p}|}+\frac{1}{|\vec{p}|} \frac{1}{\mathrm{e}^{|\vec{p}| / T}-1} .
\end{aligned}
$$


Substituindo (H.5) em (H.4) e novamente desprezando os termos independentes da temperatura temos

$$
a=\frac{1}{2} \frac{1}{d-1} \int \frac{d \Omega_{d-1}}{(2 \pi)^{d-1}} \int_{0}^{\infty} \frac{|\vec{p}|^{d-1}}{\mathrm{e}^{|\vec{p}| / T}-1} d|\vec{p}|=\frac{\mathcal{L}^{(0)}}{2},
$$

onde, no resultado acima, identificamos a pressão do campo escalar livre. Das equações (7.6), (7.10), (H.2) e (H.6) obtemos o resultado até primeira ordem

$$
\begin{aligned}
\mathcal{L}^{\text {stat. }} & =\mathcal{L}^{(0)}\left[1+\frac{1}{2}\left(d u_{\mu} u_{\nu}-\eta_{\mu \nu}\right) \tilde{h}^{\mu \nu}\right]+\cdots \\
& =\mathcal{L}^{(0)}\left[1+\frac{1}{2}\left(d \tilde{h}^{00}-\tilde{h}\right)\right]+\cdots
\end{aligned}
$$

Na segunda linha do resultado anterior a lagrangiana efetiva é expressa explicitamente no referencial de repouso do banho térmico.

Vamos considerar a contribuição de segunda ordem para $\mathcal{L}^{\text {stat }}$. Da simetria tensorial da equação 7.12 podemos ver que o resultado pode ser expresso em termos de três tensores como segue

$$
I^{\mu \nu \alpha \beta}=b_{1} u^{\mu} u^{\nu} u^{\alpha} u^{\beta}+b_{2}\left(\eta^{\mu \nu} u^{\alpha} u^{\beta}+\text { sime. }\right)+b_{3}\left(\eta^{\mu \nu} \eta^{\alpha \beta}+\text { sime. }\right)
$$

em que, devido a simetria de $I^{\mu \nu \alpha \beta}$, temos que sime. representa a simetrização em todos os índices. Projetando os dois lados da equação (H.8) e resolvendo o sistema de equações resultante obtemos

$$
\begin{aligned}
& b_{1}=-(d+2) \mathcal{L}^{(0)}+(d+2)(d+4) b \\
& b_{2}=\frac{\mathcal{L}^{(0)}}{2}-(d+2) b \\
& b_{3}=b
\end{aligned}
$$

onde

$$
b=\frac{1}{2} \frac{1}{(d-1)(d+1)} \int \frac{d \Omega_{d-1}}{(2 \pi)^{d-1}} \int_{0}^{\infty}|\vec{p}|^{d+2} S^{2}(|\vec{p}|) d|\vec{p}|
$$

$\mathrm{e}$

$$
S^{2}(|\vec{p}|)=T \sum_{n=-\infty}^{\infty} \frac{1}{\left[(2 \pi n T)^{2}+|\vec{p}|^{2}\right]^{2}}
$$


Usando a identidade

$$
\frac{1}{\left[(2 \pi n T)^{2}+|\vec{p}|^{2}\right]^{2}}=-\frac{1}{2|\vec{p}|} \frac{d}{d|\vec{p}|} \frac{1}{(2 \pi n T)^{2}+|\vec{p}|^{2}},
$$

e fazendo uma integração por partes, podemos proceder de maneira similar ao cálculo de primeira ordem, chegando ao resultado

$$
b=\frac{\mathcal{L}^{(0)}}{4} .
$$

Substituindo a equação (H.13) em (H.9) obtemos o seguinte resultado para as constantes de estruturas na equação (H.8)

$$
\begin{aligned}
b_{1} & =\frac{d(d+2)}{4} \mathcal{L}^{(0)} \\
b_{2} & =-\frac{d}{4} \mathcal{L}^{(0)} \\
b_{3} & =\frac{1}{4} \mathcal{L}^{(0)} .
\end{aligned}
$$




\section{Referências Bibliográficas}

[Abb82] L. F. Abbott. Introduction to the Background Field Method. Acta Phys.Polon., B13:33-50, 1982.

[AxZ95] P. Arnold and C. X. Zhai. The three loop free energy for high temperature QED and QCD with fermions. Phys. Rev., D51:1906-1918, 1995.

[AZ94] P. Arnold and C. X. Zhai. The three loop free energy for pure gauge QCD. Phys. Rev., D50:7603-7623, 1994.

[BD94] N. D. Birrell and P. C. W. Davies. Quantum Fields in Curved Space. Cambridge University Press, Cambridge, England, 1994.

[Bel96] M. Le Bellac. Thermal Field Theory. Cambridge University Press, Cambridge, England, 1996.

[Ber74] C. W. Bernard. Feynman rules for gauge theories at finite temperature. Phys. Rev., D9:3312-3320, 1974.

[BF93a] F. T. Brandt and J. Frenkel. The graviton selfenergy in thermal quantum gravity. Phys. Rev., D48:4940-4945, 1993.

[BF93b] F. T. Brandt and J. Frenkel. The three graviton vertex function in thermal quantum gravity. Phys. Rev., D47:4688-4697, 1993.

[BF98] F. T. Brandt and J. Frenkel. The structure of the graviton self-energy at finite temperature. Phys. Rev., D58:085012-085022, 1998.

[BF12] F. T. Brandt and J. Frenkel. Hard Thermal Loops in the n-Dimensional phi3 Theory. Braz.J.Phys., 42:425-427, 2012.

[BFM07] F. T. Brandt, J. Frenkel, and D. G. C. McKeon. General Covariant Gauge Fixing for Massless Spin-Two Fields. Phys. Rev., D76:105029-105036, 2007.

[BFMS09] F. T. Brandt, J. Frenkel, D. G. C. McKeon, and J. B. Siqueira. Resummation of infrared divergences in the free-energy of spin-two fields. Phys. Rev., D80:025024-025034, 2009.

[BFS12] F. T. Brandt, J. Frenkel, and J. B. Siqueira. Nonanalyticity of the free energy in thermal field theory. Phys.Rev., D86:107701-107703, 2012.

[BFT95] F. T. Brandt, J. Frenkel, and J. C. Taylor. High temperature QCD and the classical Boltzmann equation in curved space-time. Nucl. Phys., B437:433446, 1995. 
[BP90a] E. Braaten and R. D. Pisarski. Deducing hard thermal loops from ward identities. Nucl. Phys., B339:310-324, 1990.

[BP90b] E. Braaten and R. D. Pisarski. Soft amplitudes in hot gauge theories: A general analysis. Nucl. Phys., B337:569-634, 1990.

[BS12a] F. T. Brandt and J. B. Siqueira. Thermal Effective Lagrangian of Static Gravitational Fields. Phys. Rev., D85:067701-067705, 2012.

[BS12b] F. T. Brandt and J. B. Siqueira. The high temperature limit in static backgrounds. Phys.Rev., D86:105001-105013, 2012.

[dAO95] S. P. de Alwis and N. Ohta. Thermodynamics of quantum fields in black hole backgrounds. Phys.Rev., D52:3529-3542, 1995.

[Das93] A. Das. Field Theory: A Path Integral Approach. World Scientific, NY, USA, 1993.

[Das97] A. Das. Finite Temperature Field Theory. World Scientific, NY, USA, 1997.

[DeW65] B. S. DeWitt. Dynamical theory of groups and fields. Gordon and Breach Science Publishers, USA, 1965.

[d'I92] R. d'Inverno. Introducing Einstein's Relativity. Oxford University Press, Oxford, England 1992.

[EGH80] T. Eguchi, P. B. Gilkey, and A. J. Hanson. Gravitation, Gauge Theories and Differential Geometry. Phys.Rept., 66:213-393, 1980.

[FP67] L. D. Faddeev and V. N. Popov. Feynman diagrams for the Yang-Mills field. Phys. Lett., B25:29-30, 1967.

[FPT09] J. Frenkel, S. H. Pereira, and N. Takahashi. Hard thermal loops in static external fields. Phys. Rev., D79:085001-085004, 2009.

[FST92] J. Frenkel, A. V. Saa, and J. C. Taylor. The pressure in thermal scalar field theory to three loop order. Phys. Rev., D46:3670-3673, 1992.

[FT90] J. Frenkel and J. C. Taylor. High temperature limit of thermal QCD. Nucl. Phys., B334:199-216, 1990.

[FT92] J. Frenkel and J. C. Taylor. Hard thermal QCD, forward scattering and effective actions. Nucl. Phys., B374:156-168, 1992.

[Gol58] J. N. Goldberg. Conservation laws in general relativity. Phys. Rev., 111:315320, 1958.

[Gom02] M. O. C. Gomes. Teoria Quântica dos Campos. Edusp, SP, Brasil, 2002.

[GR80] I. S. Gradshteyn and M. Ryzhik. Tables of Integral Series and Products. Academic, NY, USA 1980.

[Kal78] R. E. Kallosh. Modified Feynman Rules in Supergravity. Nucl. Phys., B141:141-152, 1978. 
[KG06] J. I. Kapusta and Charles Gale. Finite-temperature field theory: Principles and applications. Cambridge University Press, Cambridge, England, 2006.

[KMMS09] F. C. Khanna, A. P. C. Malbouisson, J. M. C. Malbouisson, and A. E. Santana. Thermal quantum field theory - Algebraic aspects and applications. World Scientific, Singapore, 2009.

[Nie78] N. K. Nielsen. Ghost Counting in Supergravity. Nucl. Phys., B140:499-509, 1978.

[PT09] L. E. Parker and D. J. Toms. Quantum Field Theory in Curved Spacetime: Quantized Fields and Gravity. Cambridge University Press, Cambridge, England, 2009.

[Reb91] A. Rebhan. Collective phenomena and instabilities of perturbative quantum gravity at nonzero temperature. Nucl. Phys., B351:706-734, 1991.

[Sal97] S. R. Salinas. Introdução à Física Estatística. Edusp, SP, Brasil, 1997.

[TE30] R. C. Tolman and P. Ehrenfest. Temperature equilibrium in a static gravitational field. Phys. Rev., 36:1791-1798, Dec 1930.

[tHV74] G. 't Hooft and M. J. G. Veltman. DIAGRAMMAR. NATO Adv.Study Inst.Ser.B Phys., 4:177-322, 1974.

[Wei72] S. Weinberg. Gravitation and Cosmology: Principles and Applications of the General Theory of Relativity. John Wiley \& Sons, NY, USA, 1972.

[Wei08] S. Weinberg. Cosmology. Oxford University Press, Oxford, England, 2008.

[Won95] C. Y. Wong. Introduction to high-energy heavy ion collisions. World Scientific, Singapore, 1995. 